\title{
MULTINATIONAL AND DOMESTIC AGROCHEMICAL CORPORATIONS IN INDIA: AN ANALYSIS OF THE STANDARDIZATON OF CORPORATE ENVIRONMENTAL DISCLOSURES
}

\author{
By \\ Anna Jessop \\ B. Sc., Mount Saint Vincent University \\ 2016 \\ A thesis \\ presented to Ryerson University \\ in partial fulfilment of the requirements for the degree of \\ Master of Applied Science \\ in the Program of \\ Environmental Applied Science and Management \\ Toronto, Ontario, Canada, 2018 \\ (C)Anna Jessop, 2018
}




\section{Author's Declaration}

I hereby declare that I am the sole author of this thesis. This is a true copy of the thesis, including any required final revisions, as accepted by my examiners.

I authorize Ryerson University to lend this thesis to other institutions or individuals for the purpose of scholarly research.

I further authorize Ryerson University to reproduce this thesis by photocopying or by other means, in total or in part, at the request of other institutions or individuals for the purpose of scholarly research. I understand that my thesis may be made electronically available to the public. 


\begin{abstract}
MULTINATIONAL AND DOMESTIC AGROCHEMICAL CORPORATIONS IN INDIA: AN ANALYSIS OF THE STANDARDIZATON OF CORPORATE ENVIRONMENTAL DISCLOSURES

Anna Jessop

Master of Applied Science, 2018

Environmental Applied Science and Management

Ryerson University
\end{abstract}

Multinational corporations operating in developed countries are leaders in corporate environmental disclosure (CED), this often isn't true for subsidiaries operating in developing countries. The majority of CED research that has been conducted focuses primarily on large multinational corporations, leaving a gap of knowledge regarding the subsidiary operations of multinational corporations. This study provides insight into whether or not multinational corporations are implementing comprehensive disclosure practices throughout the entirety of their operations and if reporting practices are more strongly influenced by country of origin or country of operation. Consolidated narrative interrogation (CONI) is used to quantify CEDs presented in annual and stand-alone sustainability reports published between 2002 and 2016 by companies from three categories of corporations. Results show that the corporation category is a significant factor affecting the diversity, quantity and quality of disclosures, indicating a lack of standardization among the reporting practices of the different categories of corporations. 


\section{Acknowledgements}

I would like to acknowledge the assistance and guidance provided by my supervisors Dr. Michal Bardecki and Dr. Cory Searcy, who have both provided vast amounts of knowledge and mentorship throughout the development of my thesis. As well as my defence committee, including Dr. Kelly Mackay and Dr. Ron Pushchak and defence chair, Dr. Deborah de Lange; whose review of my thesis brought a new perspective and further development. I would also like to thank Dr. Andrew Laursen for his assistance throughout my analysis and statistical work. Additionally, I want to acknowledge the support of the Ontario Graduate Scholarship Program as well as Ryerson University for the financial support which made this research possible.

Furthermore, I am grateful for the support of my family, friends and classmates for the encouragement, motivation and accountability that enabled the continuous effort required to complete this project. 


\section{Table of Contents}

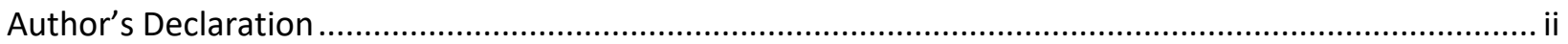

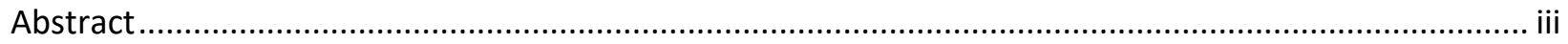

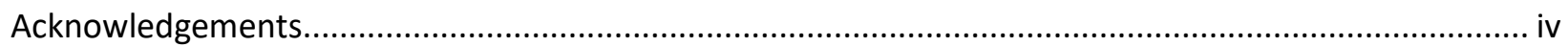

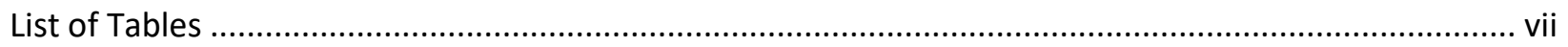

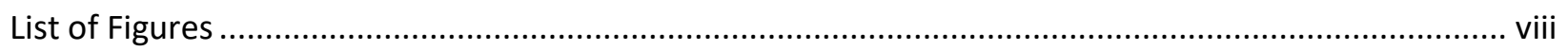

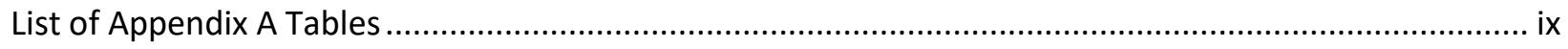

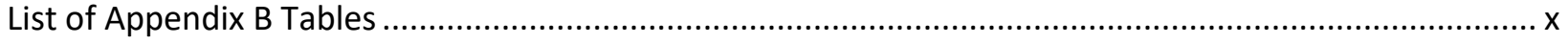

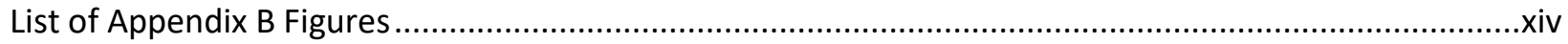

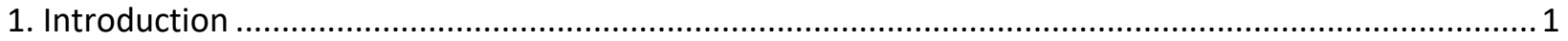

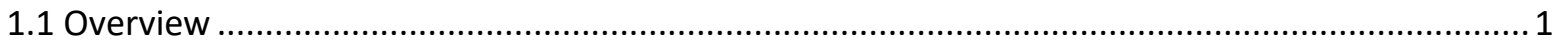

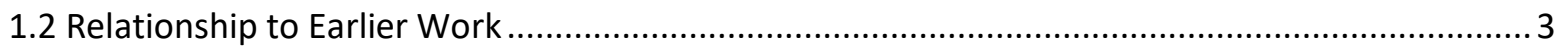

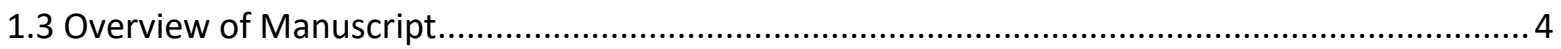

2. Multinational and Domestic Agrochemical Corporations in India: An Analysis of the Standardization of Corporate Environmental Disclosures .......................................................................................... 5

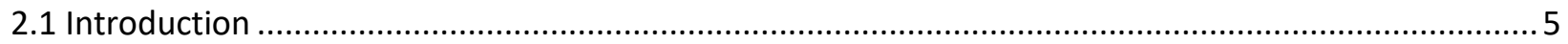

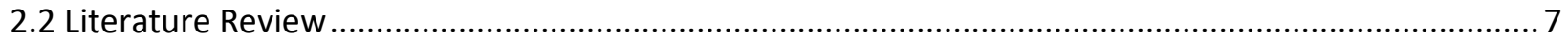

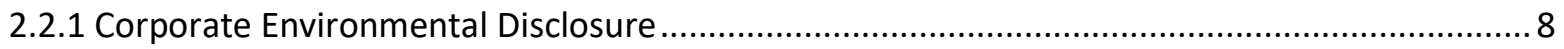

2.2.2 History of Corporate Environmental Disclosures.................................................................... 10

2.2.3 Corporate Environmental Disclosure in India .......................................................................... 11

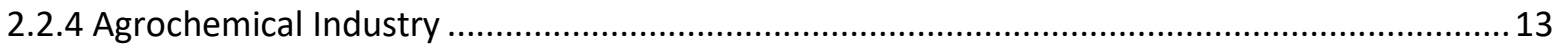

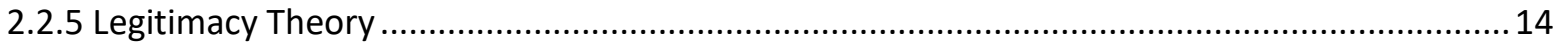

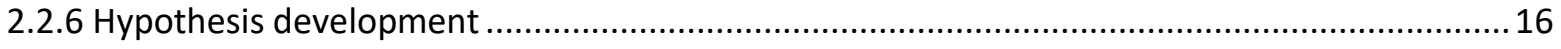

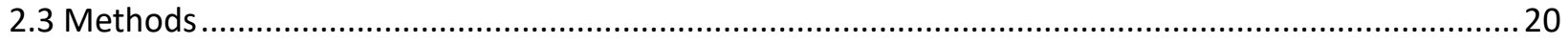

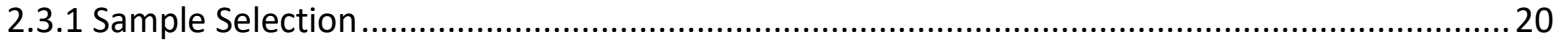

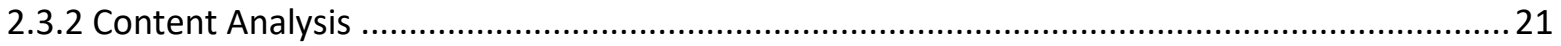

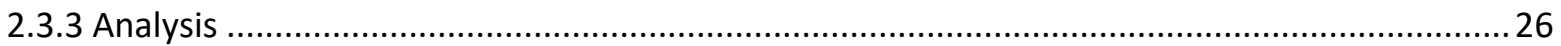

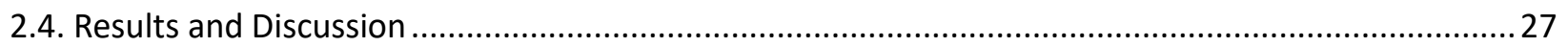

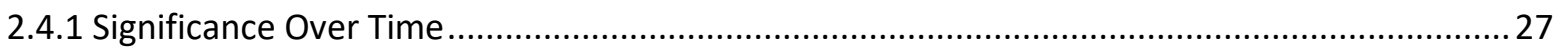

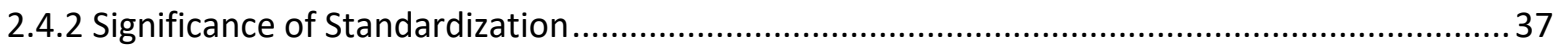

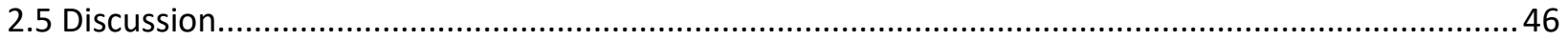

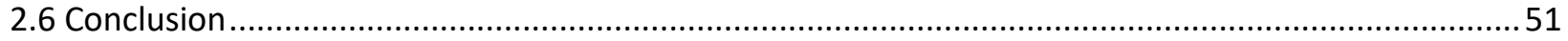




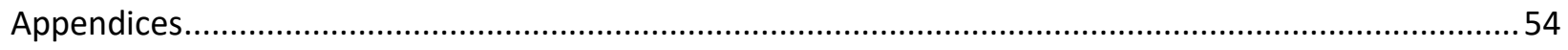

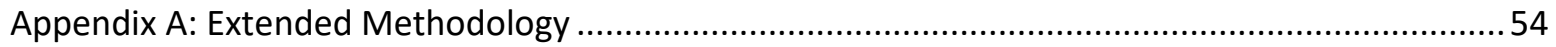

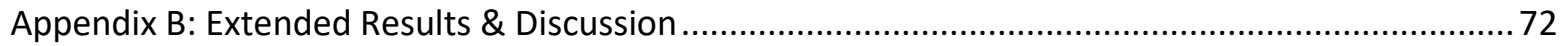

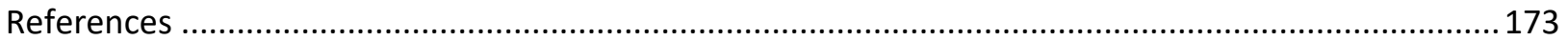




\section{List of Tables}

Table 1: Profiles of corporations selected for research ........................................................................21

Table 2: Diversity coding categories and sub-categories for CONI diversity analysis...............................24

Table 3: Examples of Quality type disclosure classifications ..............................................................26

Table 4: Diversity scores for CEDs from domestic corporations, I-MNC and P-MNC for the years 2002-

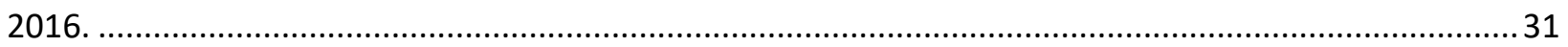

Table 5: Quantity of CEDs from domestic corporations, I-MNC and P-MNC for the years 2002-2016.....33

Table 6. Quality type by percent for CEDs from domestic corporations, I-MNC and P-MNC for the years

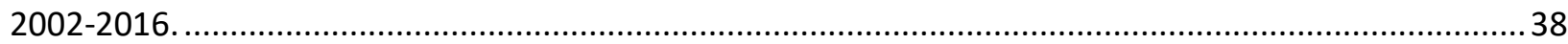




\section{List of Figures}

Figure 1. Total diversity score of disclosure for domestic Indian corporations from 2002-2016.

Coromandel, Rallis, UPL, Zuari

Figure 2. Total diversity score of disclosure for I-MNCs from 2002-2016. I-BASF, I-Bayer, I-Monsanto, ISyngenta.

Figure 3. Total diversity score of disclosures from 2002-2016. P-BASF, P-Bayer, P-Monsanto, P-Syngent.

Figure 4. Mean diversity score of disclosures from domestic Indian corporations, I-MNCs and P-MNCs

from 2002-2016. .. .32

Figure 5. Total quantity of disclosures for domestic Indian corporations from 2002-2016 ......................35

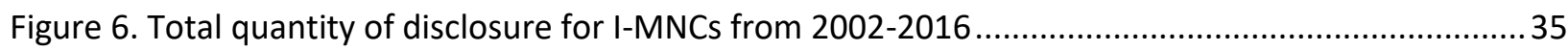

Figure 7. Total quantity of disclosure for P-MNCs from 2002-2016 .................................................... 35

Figure 8. Mean quantity of disclosure for domestic Indian corporations, I-MNCs and P-MNC for 2002-

2016. 35

Figure 9. Quality composition of disclosures from Coromandel from 2002-2016............................... 40

Figure 10. Quality composition of disclosures from Rallis India Ltd from 2002-2016...........................40

Figure 11. Quality composition of disclosure from UPL from 2002-2016. ...........................................40

Figure 12. Quality composition of disclosure from Zuari from 2002-2016. .......................................... 40

Figure 13. Quality composition of disclosure from I-BASF from 2002-2016. .......................................41

Figure 14. Quality composition of disclosure from I-Bayer from 2002-2016.......................................41

Figure 15. Quality composition of disclosure from I-Monsanto from 2002-2016................................. 41

Figure 16. Quality composition of disclosure from I-Syngenta from 2002-2016 .................................. 41

Figure 17. Quality composition of disclosure from P-BASF from 2002-2016........................................42

Figure 18. . Quality composition of disclosure from P-Bayer from 2002-2016. .................................... 42

Figure 19. Quality composition of disclosure from P-Monsanto from 2002-2016................................ 42

Figure 20. Quality composition of disclosure from P-Syngenta from 2002-2016................................42 


\section{List of Appendix A Tables}

Table A. 1: Profiles of Corporations Selected for Research .................................................................5 54

Table A. 2: Length of annual reports published by selected corporations in number of pages.................55

Table A. 3: Overview of Standalone sustainability reports published by corporations indicated by page

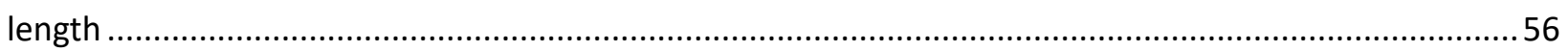

Table A. 4: Category and sub-category of diversity coding with examples ..........................................58

Table A. 5: Classification categories for qualitative coding …...............................................................68 


\section{List of Appendix B Tables}

Table B. 1: Regression statistics for the diversity of CEDs from Coromandel from 2002-2016 ................74

Table B. 2: Regression statistics for the diversity of CEDs from Rallis from 2002-2016 ........................... 75

Table B. 3: Regression statistics for the diversity of CEDs from UPL from 2002-2016 ............................75

Table B. 4: Regression statistics for the diversity of CEDs from Zuari from 2002-2016 ..........................76

Table B. 5: Regression statistics for the diversity of CEDs from I-BASF from 2002-2016 ........................78

Table B. 6: Regression statistics for the diversity of CEDs from I-Bayer from 2002-2016 ........................78

Table B. 7: Regression statistics for the diversity of CEDs from I-Monsanto from 2002-2016..................79

Table B. 8: Regression statistics for the diversity of CEDs from I-Syngenta from 2002-2016 ..................79

Table B. 9: Regression statistics for the diversity of CEDs from P-BASF from 2002-2016 ........................81

Table B. 10: Regression statistics for the diversity of CEDs from P-Bayer from 2002-2016 ......................82

Table B. 11: Regression statistics for the diversity of CEDs from P-Monsanto from 2002-2016...............82

Table B. 12: Regression statistics for the diversity of CEDs from P-Syngenta from 2002-2016 ................83

Table B. 13: Regression statistics for the diversity of CEDs from Domestic Indian Corporations from 2002-

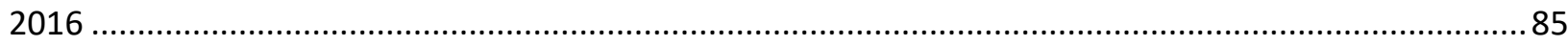

Table B. 14: Regression statistics for the diversity of CEDs from Indian Subsidiary Corporations from

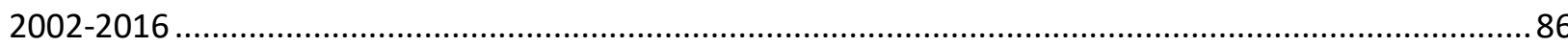

Table B. 15: Regression statistics for the diversity of CEDs from Parent Multinational Corporations from

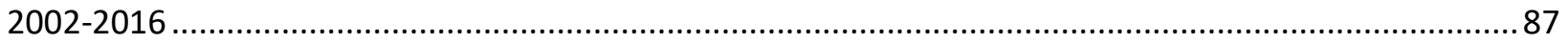

Table B. 16: Regression statistics for the quantity of CEDs from Coromandel from 2002-2016...............91

Table B. 17: Regression statistics for the quantity of CEDs from Rallis from 2002-2016 ........................91

Table B. 18: Regression statistics for the quantity of CEDs from UPL from 2002-2016 ........................ 92

Table B. 19: Regression statistics for the quantity of CEDs from Zuari from 2002-2016 .......................92

Table B. 20: Regression statistics for the quantity of CEDs from I-BASF from 2002-2016 .......................94

Table B. 21: Regression statistics for the quantity of CEDs from I-Bayer from 2002-2016 .....................94

Table B. 22: Regression statistics for the quantity of CEDs from I-Monsanto from 2002-2016................95

Table B. 23: Regression statistics for the quantity of CEDs from I-Syngenta from 2002-2016 .................95

Table B. 24: Regression statistics for the quantity of CEDs from P-BASF from 2002-2016 ......................98

Table B. 25: Regression statistics for the quantity of CEDs from P-Bayer from 2002-2016 .....................98

Table B. 26: Regression statistics for the quantity of CEDs from P-Monsanto from 2002-2016 ...............98

Table B. 27: Regression statistics for the quantity of CEDs from P-Syngenta from 2002-2016 ................99

Table B. 28: Regression statistics for the quantity of CEDs from domestic Indian corporations from 2002-

2016

Table B. 29: Regression statistics for the quantity of CEDs from Indian subsidiary corporations from

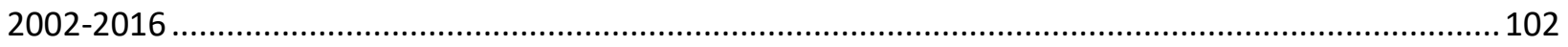

Table B. 30: Regression statistics for the quantity of CEDs from parent multinational corporations from 2002-2016. 103

Table B. 31: Regression statistics for the proportion of quality type 1 of CEDs from Coromandel from 2002-2016 104

Table B. 32: Regression statistics for the proportion of quality type 2 of CEDs from Coromandel from 2002-2016 105

Table B. 33: : Regression statistics for the proportion of quality type 3 of CEDs from Coromandel from 2002-2016. 105 
Table B. 34: Regression statistics for the proportion of quality type 4 of CEDs from Coromandel from 2002-2016

Table B. 35: Regression statistics for the proportion of quality type 5 of CEDs from Coromandel from 2002-2016 106

Table B. 36: Regression statistics for the proportion of quality type 1 of CEDs from Rallis India Ltd from 2002-2016 107

Table B. 37: Regression statistics for the proportion of quality type 2 of CEDs from Rallis India Ltd from 2002-2016 108

Table B. 38: Regression statistics for the proportion of quality type 3 of CEDs from Rallis India Ltd from 2002-2016 108

Table B. 39: Regression statistics for the proportion of quality type 4 of CEDs from Rallis India Ltd from 2002-2016 109

Table B. 40: Regression statistics for the proportion of quality type 5 of CEDs from Rallis India Ltd from 2002-2016 109

Table B. 41: Regression statistics for the proportion of quality type 1 of CEDs from UPL from 2002-2016

Table B. 42: : Regression statistics for the proportion of quality type 2 of CEDs from UPL from 2002-2016

Table B. 43: Regression statistics for the proportion of quality type 3 of CEDs from UPL from 2002-2016

Table B. 44: Regression statistics for the proportion of quality type 4 of CEDs from UPL from 2002-2016

Table B. 45: Regression statistics for the proportion of quality type 5 of CEDs from UPL from 2002-2016

Table B. 46: Regression statistics for the proportion of quality type 1 of CEDs from Zuari from 2002-2016

Table B. 47: Regression statistics for the proportion of quality type 2 of CEDs from Zuari from 2002-2016

Table B. 48: Regression statistics for the proportion of quality type 3 of CEDs from Zuari from 2002-2016

Table B. 49: Regression statistics for the proportion of quality type 4 of CEDs from Zuari from 2002-2016

Table B. 50 : Regression statistics for the proportion of quality type 5 of CEDs from Zuari from 2002-2016

Table B. 51: Regression statistics for the proportion of quality type 1 of CEDs from I-BASF from 20022016.

Table B. 52: Regression statistics for the proportion of quality type 2 of CEDs from I-BASF from 20022016

Table B. 53: Regression statistics for the proportion of quality type 3 of CEDs from I-BASF from 20022016

Table B. 54: Regression statistics for the proportion of quality type 4 of CEDs from I-BASF from 20022016

Table B. 55: Regression statistics for the proportion of quality type 5 of CEDs from I-BASF from 20022016 
Table B. 56: Regression statistics for the proportion of quality type 1 of CEDs from I-Bayer from 20022016

Table B. 57: Regression statistics for the proportion of quality type 2 of CEDs from I-Bayer from 20022016

Table B. 58: Regression statistics for the proportion of quality type 3 of CEDs from I-Bayer from 20022016

Table B. 59: Regression statistics for the proportion of quality type 4 of CEDs from I-Bayer from 20022016

Table B. 60: Regression statistics for the proportion of quality type 5 of CEDs from I-Bayer from 2002-

2016

Table B. 61: Regression statistics for the proportion of quality type 1 of CEDs from I-Monsanto from 2002-2016

Table B. 62: Regression statistics for the proportion of quality type 2 of CEDs from I-Monsanto from 2002-2016

Table B. 63: Regression statistics for the proportion of quality type 3 of CEDs from I-Monsanto from 2002-2016

Table B. 64: Regression statistics for the proportion of quality type 4 of CEDs from I-Monsanto from 2002-2016

Table B. 65: Regression statistics for the proportion of quality type 5 of CEDs from I-Monsanto from 2002-2016

Table B. 66: Regression statistics for the proportion of quality type 1 of CEDs from I-Syngenta from 20022016

Table B. 67: Regression statistics for the proportion of quality type 2 of CEDs from I-Syngenta from 20022016

Table B. 68: Regression statistics for the proportion of quality type 3 of CEDs from I-Syngenta from 20022016

Table B. 69: Regression statistics for the proportion of quality type 4 of CEDs from I-Syngenta from 20022016

Table B. 70: Regression statistics for the proportion of quality type 5 of CEDs from I-Syngenta from 20022016

Table B. 71: Regression statistics for the proportion of quality type 1 of CEDs from P-BASF from 20022016

Table B. 72: Regression statistics for the proportion of quality type 2 of CEDs from P-BASF from 20022016

Table B. 73: Regression statistics for the proportion of quality type 3 of CEDs from P-BASF from 20022016

Table B. 74: Regression statistics for the proportion of quality type 4 of CEDs from P-BASF from 20022016

Table B. 75: Regression statistics for the proportion of quality type 5 of CEDs from P-BASF from 20022016

Table B. 76: Regression statistics for the proportion of quality type 2 of CEDs from P-Bayer from 20022016

Table B. 77: Regression statistics for the proportion of quality type 3 of CEDs from P-Bayer from 20022016 
Table B. 78: Regression statistics for the proportion of quality type 4 of CEDs from P-Bayer from 20022016

Table B. 79: Regression statistics for the proportion of quality type 5 of CEDs from P-Bayer from 2002-

2016

Table B. 80: Regression statistics for the proportion of quality type 1 of CEDs from P-Monsanto from 2002-2016

Table B. 81: Regression statistics for the proportion of quality type 2 of CEDs from P-Monsanto from 2002-2016

Table B. 82: Regression statistics for the proportion of quality type 3 of CEDs from P-Monsanto from 2002-2016

Table B. 83: Regression statistics for the proportion of quality type 4 of CEDs from P-Monsanto from 2002-2016

Table B. 84: Regression statistics for the proportion of quality type 5 of CEDs from P-Monsanto from 2002-2016

Table B. 85: Regression statistics for the proportion of quality type 1 of CEDs from P-Syngenta from 2002-2016

Table B. 86: Regression statistics for the proportion of quality type 2 of CEDs from P-Syngenta from 2002-2016

Table B. 87: Regression statistics for the proportion of quality type 3 of CEDs from P-Syngenta from 2002-2016

Table B. 88: Regression statistics for the proportion of quality type 4 of CEDs from P-Syngenta from 2002-2016

Table B. 89: Regression statistics for the proportion of quality type 5 of CEDs from P-Syngenta from 2002-2016

Table B. 90: Intra-Coder reliability 140

Table B. 91: Inter- User Reliability 157 


\section{List of Appendix B Figures}

Figure B. 1: Diversity index score for the corporate environmental disclosures from domestic Indian corporations between the years 2002 and 2016. .73

Figure B. 2: Total diversity score of disclosure for domestic Indian corporations from 2002-2016. ..... 74 Figure B. 3: Diversity index score for the corporate environmental disclosures from Indian subsidiaries between the years 2002 and 2016. .77

Figure B. 4: Total diversity score of disclosure for I-MNCs from 2002-2016.

Figure B. 5: Diversity index score for the corporate environmental disclosures from Parent Multinational

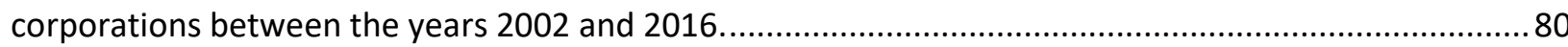

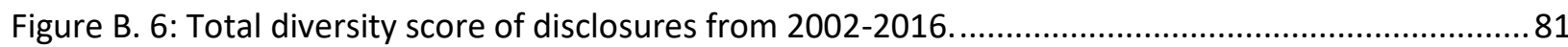

Figure B. 7: Average diversity index score for the corporate environmental disclosures from domestic corporations (Domestic), Indian subsidiary corporations (I-MNC) and parent multinational corporations (P-MNC) between the years 2002 and 2016.

Figure B. 8: Quantity of CED sentences for the corporate environmental disclosures from domestic

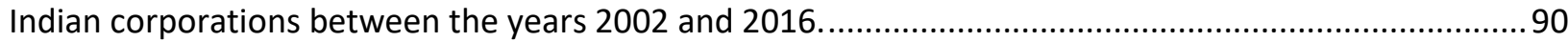

Figure B. 9: Total quantity of disclosures for domestic Indian corporations from 2002-2016................90 Figure B. 10: Quantity of CED sentences for the corporate environmental disclosures from Indian

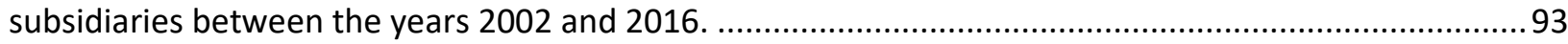

Figure B. 11: Total quantity of disclosure for I-MNCs from 2002-2016 ..............................................94

Figure B. 12: Quantity of CED sentences for the corporate environmental disclosures from parent

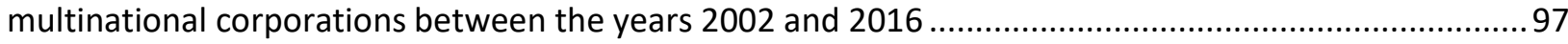

Figure B. 13: Total quantity of disclosure for P-MNCs from 2002-2016.

Figure B. 14: Total quantity of CED sentences for the corporate environmental disclosures from all companies in the category Domestic Indian corporations (Domestic), Indian subsidiary corporations (IMNC) and parents multinational corporations (P-MNC) between the years 2002 and 2016. 100 Figure B. 15: Mean quantity of disclosure for domestic Indian corporations, I-MNCs and P-MNC for 20022016. 101

Figure B. 16: Quality composition of disclosures from Coromandel from 2002-2016.......................... 104 Figure B. 17: Quality composition of disclosures from Rallis India Ltd from 2002-2016...................... 107

Figure B. 18: Quality composition of disclosure from UPL from 2002-2016...................................110

Figure B. 19: Quality composition of disclosure from Zuari from 2002-2016......................................113

Figure B. 20: Quality composition of disclosure from I-BASF from 2002-2016 ..................................... 116

Figure B. 21: Quality composition of disclosure from I-Bayer from 2002-2016.................................119

Figure B. 22: Quality composition of disclosure from I-Monsanto from 2002-2016............................ 122

Figure B. 23: Quality composition of disclosure from I-Syngenta from 2002-2016............................. 125

Figure B. 24: Quality composition of disclosure from P-BASF from 2002-2016. ................................128

Figure B. 25: Quality composition of disclosure from P-Bayer from 2002-2016................................131

Figure B. 26: Quality composition of disclosure from P-Monsanto from 2002-2016............................. 134

Figure B. 27: Quality composition of disclosure from P-Syngenta from 2002-2016............................ 137 


\section{Introduction}

\subsection{Overview}

Corporate environmental disclosures (CEDs) are publications made by corporations that contain environmental information. CEDs have not been popularized for long; most developments in this area have occurred over the last 40 years (Lin, 2008; Setyorini \& Ishak, 2012). Furthermore, multinational corporations are known to be leaders in CED publications (KPMG, 2011; Lin, 2008; Park \& Ghauri, 2015). Due to this, the majority of CED research that exists has examined multinational corporations operating in developed countries.

The purpose of this study is to analyze the CEDs from three different corporate categories: (1) parent multinationals, (2) Indian subsidiaries of those multinationals, and (3) Indian domestic corporations, over a fifteen-year timeframe. This will address a recognized gap in the literature (Ali et al., 2017) and enhance understanding of how corporations in these three categories report. More specifically, this study focuses on agrochemical corporations. The reasons for focusing on corporations in this industry are the environmentally sensitive nature of the industry and the high levels of public scrutiny and expectations that influence environmental behaviour in this industry.

The research aims to: (1) investigate if CED practices are standardized across the three categories of corporations, or how reporting practices differ amongst the categories; and (2) analyze if there has been a change in this trend over time. This will be done by collecting data from the annual reports and stand-alone sustainability reports from multinational parent companies, multinational subsidiary companies, and domestic Indian corporations. Disclosures from all three categories of corporations will be compared on the basis of diversity, quality, and quantity of environmental information disclosed. Diversity is defined as the "content per theme" (Beck et al., 2010, pg. 212). Quality is defined as the level of information provided combined with the depth or detail of the 
disclosure (Beck et al., 2010, Pg. 213). Quantity is defined as the total volume (Beck et al., 2010). The following hypotheses will be tested:

H1a: Each corporation categories studied will increase the diversity of environmental disclosures over the timeframe examined.

H1b: Each corporation categories studied will increase the quantity of environmental disclosures over the timeframe examined.

H1c: Each corporation categories studied will increase the quality of environmental disclosures over the timeframe examined.

H2a: The diversity of environmental disclosures of P-MNCs and I-MNCs will lack standardization, and the environmental disclosures of I-MNCs will be more similar to those of domestic corporations than the P-MNC.

H2b: The quantity of environmental disclosures of P-MNCs and I-MNCs will lack standardization, and the environmental disclosures of I-MNCs will be more similar to those of domestic corporations than the PMNC.

H2c: The quality of environmental disclosures of P-MNCs and I-MNCs will lack standardization, and the environmental disclosures of I-MNCs will be more similar to those of domestic corporations than the PMNC.

Data will be collected from the CEDs of twelve different corporations, four from each of the three corporate categories. The three categories are: (1) parent multinational corporations (P-MNC), which are the headquarter operations of a multinational agrochemical corporation, (2) Indian subsidiary corporations (I-MNC), which are the subsidiary corporations of the multinational parent corporation 
operating in India, and (3) domestic corporations, which are national corporations that operate in India in the agrochemical industry.

In order to collect data from the CED publications, consolidated narrative interrogation (CONI) will be used, which is a content analysis methodology developed by Beck et al. (2010). This methodology is specialized for the analysis of environmental information. Publicly-available reports from each corporation over the period 2002-2016 will provide measurements on the diversity, quantity, and quality of information reported in CEDs.

Following analysis for the changes over time and standardization of reporting practices between the corporations, it is found that all corporations increased the quantity of their disclosures over time and that P-MNC and domestic corporations had significant changes in the diversity of reporting over time. Results also showed that the corporation category was a significant factor affecting the diversity, quantity, and quality of disclosures, indicating that there is a lack of standardization across the reporting practices of the different categories of corporations. The data suggests that, in terms of diversity and quantity, subsidiary corporations conform more to localized reporting practices. The findings on standardization were inconclusive for the analysis of quality.

\subsection{Relationship to Earlier Work}

This research builds on data collected and analysis by Wilson (2013). By including data collected by Wilson along with the original data collected for this thesis, it was possible to examine reporting trends over a longer period of time than would have otherwise been possible. The data presented in Wilson's (2013) study included the timeframe 2002-2011. This study extends that timeframe to include 2012-2016. When analyzing corporate environmental disclosure for the same sample between 2002 and 2011, Wilson (2013) found that the disclosures of Indian subsidiary companies were localized to Indian norms rather than standardized within the multinational corporation. The focus of this research will be the change in the diversity, quantity, and quality of CEDs over time and will also look at the 
standardization of corporate environmental disclosures, which overlaps with Wilson's scope to an extent. Wilson's research used the same companies and methods as the research presented here and also examined the standardization between the three categories of corporations. However, this research focused more extensively on time series analysis. With the extended timeframe, this study analyses the significance of the change in reporting practices over time. The analysis of standardization also accounts for change over time by using a repeated measures ANOVA as opposed to the t-test used by Wilson. Moreover, this study introduces a theoretical framework to support the results. This thesis also updates the literature review from Wilson (2013), which is important given the rapid growth of the literature focused on CEDs.

\subsection{Overview of Manuscript}

This thesis is presented in a manuscript style, featuring this introductory chapter, along with a manuscript intended for publication composing the body of the thesis. It is anticipated the manuscript will be submitted to the Journal of Cleaner Production. Additional details regarding methods and results are published in the appendices at the end of the thesis. Only the core methods and results will be featured in the manuscript.

The authorship of the manuscript will include the thesis author (A. Jessop), thesis supervisors (Dr. M. Bardecki \& Dr. C. Searcy) and N. Wilson. As the thesis author, my role in this research was coding CEDs from 2012-2016 for the twelve corporations, combining this with Wilson's data set from 20022011, as well as performing a new and separate data analysis (as described above), which I then used to compose the manuscript in the next section.

The purpose and benefit of using a manuscript style thesis is to seamlessly combine the two sets of data into a paper publishable in an international peer-reviewed journal. The analysis and findings based on the combined sets of data are stronger than would have been possible using one of the data sets

alone. 


\title{
2. Multinational and Domestic Agrochemical Corporations in India: An Analysis of the Standardization of Corporate Environmental Disclosures
}

\begin{abstract}
Multinational corporations operating in developed countries are leaders in corporate environmental disclosure (CED), this often is not true for subsidiaries operating in developing countries. The majority of CED research that has been conducted focuses primarily on large multinational corporations, leaving a gap of knowledge regarding the subsidiary operations of multinational corporations. This study provides insight into the extent to which multinational corporations implement comprehensive disclosure practices throughout the entirety of their operations and if reporting practices are more strongly associated with the practice of parent companies or the country of operation. Consolidated narrative interrogation (CONI) is used to quantify CEDs presented in annual and stand-alone sustainability reports published between 2002 and 2016 by agrochemical companies operating in India. Results show that the corporation category is a significant factor affecting the diversity, quantity and quality of disclosure, indicating a lack of standardization among the reporting practices of the different categories of corporations.

Keywords:

Corporate environmental disclosure

Agrochemical industry

India

Subsidiaries

Legitimacy Theory

Domestic
\end{abstract}

\subsection{Introduction}

Multinational corporations are leaders in producing corporate environmental disclosures, however, this is often not true for multinational subsidiaries operating in developing countries (KPMG, 2011).

Corporate environmental disclosures have been produced by corporations since the 1980s (Lin, 2008;

Ingram \& Frazier, 1980), and when utilized properly can result in many benefits for corporations and investors (Hu \& Karbharl, 2015). Furthermore, benefits of a standardized approach to environmental disclosures within multinational corporations can include better performance targets for subsidiaries, improved policy and auditing for subsidiaries, as well as more comparable information for stakeholders (Christmann, 2004). Standardized disclosure practices can also improve corporate legitimacy, leading to 
higher profits, better stock prices, lower pressure from non-government organizations and reduced liability of foreignness (Cho \& Patten, 2007; Hunter \& Bansal, 2006).

Despite this, the majority of CED research that has been conducted focuses primarily on large multinational corporations, such as the Fortune 500 companies or the top listed corporations in a selected country (Kolk \& Pinkse, 2010; KPMG, 2011; Wilson, 2013). Studies examining corporate environmental disclosure of multinationals rarely include subsidiary corporations and studies focused on domestic corporations rarely make a distinction between domestic corporations and foreign-operated multinational subsidiaries (Wilson, 2013).

This research aims to: a) analyze the diversity, quantity and quality of corporate environmental disclosures from domestic corporations, subsidiary corporations and multinational corporations to identify trends over a fifteen-year timeframe, and b) determine if corporate environmental disclosures within the agrochemical industry from the three categories of corporations are standardized. This is being done by collecting data from the annual reports and stand-alone sustainability reports from three categories of corporations: multinational parent companies (P-MNC), multinational subsidiary companies (I-MNC), and domestic Indian corporations. Multinational parent corporations refer to the headquarter operation of a multinational agrochemical corporation, while the multinational subsidiary companies are subsidiary corporations of the same multinational corporation which are operating in India. In addition, domestic corporations will also be examined; these corporations are national publically traded corporations which operate in India in the agrochemical industry.

To achieve this, the content of annual reports and sustainability reports published between the year 2002 and 2016 are quantified using Consolidated Narrative Interrogation (CONI). This comprehensive approach to content analysis measures material on diversity, quantity and quality. The data are analyzed using simple linear regression and two way repeated measures ANOVA. 
This research finds that there is a lack of standardization across the reporting practices of the three categories of corporations. Results show that I-MNCs are reporting in a way that is more similar to domestic corporations, suggesting I-MNCs follow a localized reporting strategy. Additionally, the results show that the diversity of CEDs significantly increased for P-MNCs and domestic corporations and that the quantity of CEDs significantly increased for all corporations over the fifteen-year timeframe. The results for CED quality were inconclusive.

These findings are significant in several ways and contribute to academic research and future decision making regarding environmental disclosure policy and regulation. The existing literature focuses primarily on the environmental disclosures of large multinational corporations, with relatively little research addressing the reporting of the subsidiaries of those multinationals. This research is a step towards addressing this gap by producing findings for the primary and subsidiary operations of multinational corporations, as well as domestic corporations. This information is likely to be useful in future policy and regulation decisions around environmental disclosure. Currently, there is an ongoing discussion in many countries regarding the necessity of implementing mandatory environmental disclosure, particularly in the case of large multinational corporations (KPMG, 2017). Understanding how international subsidiary reports compare to multinational corporations and domestic corporations can aid in implementing effective regulation for companies whose operations span multiple countries.

\subsection{Literature Review}

This literature review will introduce relevant literature on corporate environmental disclosures, environmental regulations in India, the agrochemical industry, and legitimacy theory in order to establish the scope and relevance of this research. 


\subsubsection{Corporate Environmental Disclosure}

Corporate environmental disclosures (CEDs) consist of both mandatory and voluntary reporting of information relating to the environment (Villiers \& van Staden, 2011). CEDs developed as a result of increasing stakeholder and public concern regarding the environmental impacts of corporations (Hughes et al., 2001; Sumiani et al., 2007). There are several ways in which a corporation can communicate the impact of corporate activities on the environment to stakeholders, including in annual reports, standalone sustainability reports, websites and promotional publications (Carreira \& Abreu, 2014; Trabelsi et al., 2013; Villiers \& van Staden, 2011).

There are several perceived benefits of environmental disclosure, which drive corporations to publicly report. By disclosing, corporations have the opportunity to improve their reputation, which in turn can improve competitive advantage, increase profit margins, attract investors and increase the potential sales market (Hu \& Karbharl, 2015). These corporations can also benefit from cost-savings, due to more efficient use of resources and reduced risk of legal actions or public slandering (Hu \& Karbharl, 2015). Social and economic factors, such as increased profits and brand recognition, have become significant considerations for corporations regarding voluntary disclosures (Buhr \& Freedman, 2001; Sumiani et al. 2007; Villiers \& van Staden, 2011). Cormier and Magnan (1999) define the phenomenon of information costs, where if a corporation fails to provide credible information on a topic, investors and stakeholders will assume the worst. In fact, disclosing environmental information can impact many aspects of a corporation, which is why corporations tend to employ disclosures as part of a strategic plan (Cormier \& Magnan 1999; Villiers \& van Staden, 2011).

There are also several reasons why a corporation may opt to not disclose environmental information, including not believing the costs outweigh the benefits, the absence of legislation and regulation, or little interest from investors and stakeholders (Hu \& Karbharl, 2015; Stubbs et al., 2013). In some cases, 
corporations can even harm their reputation with the information disclosed, (Cho \& Patten, 2007; Hu \& Karbharl, 2015; Mousa \& Hassan, 2015). When there are very few legal requirements or regulating bodies, companies may take the opportunity to disclose inaccurate information or misrepresent information, which may lead to the greenwashing of the firm (Hahn \& Lülfs, 2014). Firms publishing disclosures in an environment with more readily enforced legal requirements, have a tendency to disclose less information than others (Cormier \& Magnan, 1999). This indicates that in the absence of regulation, corporations may take advantage of the lack of monitoring and use environmental disclosures that lack evidence of environmental sustainability as a way to promote themselves. Foreign ownership has been researched for its effects on CED with conflicting results. Several studies have suggested that companies having foreign ownership based in developed countries whilst the corporation itself is operating in a country with a developing economy, may increase the level of CED published by that corporation (Khan et al., 2013; Khilf et al., 2017; Malarvizhi, 2008; Oh et al., 2011; Özen \& Küskü, 2009). This is based on the idea that foreign owners influence a corporation's environmental standards. Other studies have found that there is no impact or a negative impact of foreign ownership on CEDs (Chaklader \& Gulati, 2015; Haladu \& Salim, 2016; Hossain \& Andrew, 2006; Monteiro \& Aibar-Guzman, 2010; Zeng et al. 2012). The reasoning behind results that show reduced disclosure from foreign-owned corporations is unclear. Though some researchers have suggested that the lack of increased disclosure from foreign-owned companies relates to the voluntary nature of environmental disclosure (Haladu \& Salim, 2016), others state that no there is no explanatory power and indicate that significant impact of ownership on CEDs was expected (Chaklader \& Gulati, 2015; Zeng et al., 2012).

Many of the drivers for companies to disclose environmental information correspond to legitimacy theory (Cho \& Patten, 2007; Mousa \& Hassan, 2015). This theory describes the legitimization process that many researchers credit for the development of environmental disclosure by corporations (Mousa 
\& Hassan, 2015). Legitimacy theory is based on pressure from society and therefore tends to affect corporations in industries with higher scrutiny more intensely (Cho \& Patten, 2007). This provides a rationale for corporations to spend money producing environmental disclosures but also presents the motivation for corporations to report information in a way that may be misleading (Cho \& Patten, 2007; Mousa \& Hassan, 2015). Due to this, legitimacy theory has been blamed for creating an unreliable relationship between environmental disclosure and environmental performance (Cho \& Patten, 2007; Hummel \& Schlick, 2016). Himmel and Schlick (2016) also suggest that the quality of disclosure can be used to identify superior performing corporations from corporations seeking legitimization.

\subsubsection{History of Corporate Environmental Disclosures}

Corporate social responsibility was first discussed at length by Howard Bowen in the 1950s, where he wrote about the connection between business decisions and quality of life of the general society, this greatly influenced society's expectation for corporate activity (Bowen, 1953; Carrol, 1999). In the 1960s it became clear that a significant portion of the general public was concerned by environmental wellbeing, and the impact of corporations and industrial activities (Buhr \& Freedman, 2001; Carrol, 1999). This was likely due to a number of high-profile environmental disasters around that time, including the great London smog of 1952 (Davis et al., 2002), Minamata disease in the late 1950s (Harada, 1995) and the publication of Rachel Carson's Silent Spring in 1962 (Long, 2000). In the 1970s the idea of corporate environmental disclosure began gaining recognition (Mathews, 1997). Following this, select corporations began using annual reports and other publications to report environmental information, realizing that such information could improve a company's image, while influencing stakeholders (Setyorini \& Ishak, 2012). By the start of the 1980s, almost all large corporations included a brief acknowledgment of environmental performance as a part of their annual report (Lin, 2008). Also during this time, the idea of 
an ethical investor began to form, going against the previous idea that investors did not want corporations to participate in corporate social responsibility activities for the reason that it reduced shareholders' equity (Bowman \& Haire, 1975; Belkaoui, 1976).

By the 1990s the quantity of environmental disclosure published in annual reports had increased significantly for the majority of large corporations (Lin, 2008). Stakeholders began to harbour concerns regarding the accuracy of information being reported and encouraged imposing reporting requirements (Rockness, 1985). The next two decades of research were focused primarily on assessing the quality and quantity of disclosures (Lin, 2008; Sand \& Van Buren, 1990). Additionally, the Global Reporting Initiative (GRI) was released in 1997 addressing stakeholder concerns and helped lead to an increase in the transparency and quality of disclosures (Malarvizhi, 2008).

From the early 2000s to today, CED research has branched out into several streams, Wilson (2013) identifies four main areas of research, including impact of environmental disclosure on financial performance (Hughes et al., 2001), the publication location of disclosures (i.e. annual reports, websites, advertisement) (Villiers \& van Staden, 2011), the effect of external pressures on quality and quantity of disclosures (Iatridis, 2013; Islam \& Islam, 2011), and the relationship between environmental disclosures and environmental performance (Meng et al., 2014).

\subsubsection{Corporate Environmental Disclosure in India}

In India, the Ministry of Environment and Forests (MOEF) is the primary entity responsible for presiding over environmental preservation and conservation (Chaklader \& Gulati, 2015). The MOEF has implemented several pieces of legislation to help meet the responsibilities of this position, including the Water Act, the Air Act and the Environment Act (Chaklader \& Gulati, 2015). Despite this, India has experienced several notable environmental disasters, including the Bhopal disaster (Yang et al., 2014), the Pasarlapudi blowout (Srivastav et al., 1998), and Bellandur Lake (Samal et al., 2011). The Bhopal 
disaster is perhaps the most notable, as it is commonly referred to as one of the world's worst industrial disasters (Broughton, 2005; Chouhan, 2005; Stix, 1989).

Until 2015, all environmental disclosures from corporations operating in India were voluntary, with the exception of submitting a statement to the pollution control board (Sen et al., 2011). Although many larger corporations operating in India do report some environmental information, there is an abundance of criticism regarding these reports (Chaklader \& Gulati, 2015). This includes the tendency of corporations to report exclusively on positive environmental aspects while dismissing negative impacts (Malarvizhi, 2008). From 2015 onwards, the Indian government made CSR disclosures mandatory through the Companies Act 2013 for corporations with revenue in excess of 10 billion rupees to publish information regarding their corporate social responsibility policy, though not necessarily environmental information (The Companies Act, 2013; KPMG, 2017). Information that the Act does require includes details regarding a CSR committee and their responsibilities, a CSR board statement, and a CSR policy, failure to do is punishable by fines (Deodhar, 2016). Environmental sustainability fits into the accepted streams for CSR disclosure and therefore the Act may influence corporations to disclose more environmental information. However, corporations can fulfill the requirements of the Act without discussing environment by instead focusing on health, education and other charity initiatives (Deodhar, 2016). Although there are no results yet of the effects of the Act on environmental disclosures, Deodhar (2016) predicts that mandatory requirements may reduce the quality of CSR initiatives. Deodhar predicts that corporations will anchor on to the requirements of the Act, which fail to specify requirements regarding quantity or quality, and that this could impact the natural development of increasing quality (Deodhar, 2016). Alternatively, other research has suggested that mandatory regulation increases the quality and quantity of environmental disclosure from corporations (Fatima et al., 2015; loannou \& Serafeim, 2017). These studies suggest that the reason for increasing quality and quantity lies in the expectation that corporations will first and foremost strive to meet the law and 
failure to do this would significantly impact the corporations' reputation and legitimacy (Fatima et al., 2015; loannou \& Serafeim, 2017).

\subsubsection{Agrochemical Industry}

The agrochemical industry has assumed an important role in continued global food security and disease prevention (Unsworth et al., 2012). The industry is responsible for the production of pesticides, herbicides, fertilizers, phytohormones and other growth aids (Sparks \& Lorsbach, 2017; Research and Markets, 2016). Innovation in the agrochemical industry remains high, as corporations continue to develop new products to meet increasingly stringent environmental standards and predict agricultural resistance to applied substances (Sparks \& Lorsbach, 2017). The agrochemical industry, globally, is expected to be valued at USD 260 billion by the year 2021 (Research and Markets, 2016).

Despite the agrochemical industry's role in global food security and affordable food pricing, it has been responsible for environmental degradation, as well as some of the most devastating industrial disasters in history. Strict environmental standards do exist for the use of agricultural chemicals, agrochemical products undergo up to eleven years of research and testing before being introduced to the market (Sparks \& Lorsbach, 2017). Despite this, many substances are pulled off the market due to environmental or health impacts of the products years or decades after being approved without fully understanding of non-target effects (Hayes \& Hasen, 2017; Sparks \& Lorsbach, 2017). Currently, the development of new agrochemical compounds requires eleven years of research and testing, and requires almost USD 300 million of investment (Sparks \& Lorsbach, 2017). Regardless, many health and safety concerns exist around the agrochemical industry, including impacts to wildlife, water contamination, and human health effects (Aktar et al., 2009; Hayes \& Hasen, 2017).

In addition to environmental degradation, the agrochemical industry can also have adverse health effects. The agrochemical industry is connected to an estimated one million deaths globally each year, 
through chronic and acute pesticide poisoning, with up to 260,000 being intentional poisoning (Aktar et al., 2009; Mew et al., 2017). Most of these deaths occur in high-risk populations in developing countries, including primary workers in production plants and residents in rural areas (Quinteros et al., 2017; Aktar et al., 2009). General populations are also put at risk due to the lack of regulation for agrochemical disposal, specifically, the disposal of banned chemical compounds, which in developing countries are often illegally dumped by manufacturers when no longer saleable (Quinteros et al., 2017). India began producing agrochemicals in 1952 and is currently the twelfth largest producer of agrochemicals globally (Aktar et al., 2009). The Indian agrochemical industry provides products for both national use as well as exports (KPMG, 2016). In India, the agricultural industry accounts for fifteen percent of the country's GDP despite challenging growth conditions (KPMG, 2016). India has also become a preferred supplier of low-cost generic agrochemicals for non-patented compounds (KPMG, 2016).

The agrochemical industry was selected to be studied because of its prominent role in India and in India's economy. It was also selected because of the industry's potential for future growth as the agricultural industry continues to be impacted by the effects of climate change. Additionally, the CEDs from companies operating in the agrochemical industry were of particular interest because of the environmentally sensitive nature of the industry.

\subsubsection{Legitimacy Theory}

Many theories have been applied to corporate reporting in an attempt to determine the corporate motivation for reporting, including legitimacy theory, stakeholder theory, accountability theory, and agency theory (Donovan, 2002). Legitimacy theory posits that corporations maintain their legitimacy or reputation by meeting the expectation of society as a whole. Suchman (1995: 574) offered the following definition for legitimacy: "generalized perception or assumption that the actions of an entity are 
desirable, proper or appropriate within some socially-constructed system of norms, values, beliefs and definitions". This continues to be the most used definition of legitimacy, though some researchers have questioned the vagueness of the definition (Deephouse et al., 2017). Legitimacy theory, however, is one of the leading theoretical frameworks used when examining corporate disclosures, largely because corporate disclosures are the main way a corporation can demonstrate that they are fulfilling the expectations of society (Arora \& Lodhia, 2017). Legitimacy theory expects that organizations will act in a way that abides by societal norms and expectations (Braam et al., 2016; Deegan, 2002).

One way for corporations to communicate their environmental responsibility and therefore indicate that they are fulfilling society expectation is through disclosures in annual and sustainability reports (Braam et al., 2016). Consequently, corporations may also disclose information that does not accurately depict the corporations' environmental performance (Ashforth \& Gibbs, 1990; Braam et al., 2016; Cho et al. 2007). This is not always done in an attempt to greenwash corporate activities; though this certainly occurs, it can also be an attempt to secure corporate legitimacy by not drawing attention to areas of operation that may not fulfill society's expectations (Hahn \& Lülfs, 2014). As a result, the relationship between environmental reporting and environmental performance is not able to be ensured (Braam et al. 2016, Clarkson et al., 2011; Hughes et al., 2001 \& Ingram \& Frazier, 2017).

Corporate legitimacy is a highly dynamic and a valuable resource for companies, as social values and expectations are constantly changing and therefore corporations must continually change to meet and predict these expectations (Ashforth \& Gibbs, 1990). If a corporation does meet society's expectations it can gain legitimacy, which is associated with increased organizational survival (Ashford \& Gibbs, 1990; Dowling \& Pfeffer, 1975; Suddaby et al., 2017). However, because a society's expectations are everchanging, if a corporation ceases to adapt, its legitimacy can be negatively impacted. 
Furthermore, multinational corporations face increased complexity in achieving and maintaining legitimacy (Kostova \& Zaheer, 1999). This is because in addition to facing the complex institutional environment that all organizations encounter, multinationals are also faced with increased complexity within the corporation itself caused by fragmentation, operation in several institutional environments, and liability of foreignness, which refers to obstacles faced by corporations operating in a foreign market (Fiaschi et al., 2014; Kostova \& Zaheer, 1999).

\subsubsection{Hypothesis development}

The purpose of this study is to address two main points of interest. The first is to investigate if there have been significant changes in the reporting practices of select corporations over the past fifteen years. The other is to examine if there is standardization in reporting practices across the CEDs published by the three categories of corporations. To achieve this, two main hypotheses have been developed. The first posits that all corporations will increase the diversity, quantity and quality of their environmental disclosures over the timeframe examined. The second is that the environmental disclosures of foreign subsidiaries will be more similar to the disclosures of domestic corporations rather than the disclosures of the parent multinational corporation. The hypotheses are tested using content analysis to first quantify the data, then analyzed using simple linear regression and two-way repeated measures ANOVA.

The hypotheses for this study were developed based on the lack of knowledge surrounding the differing quality, quantity, and diversity of corporate environmental disclosures (CEDs) of multinational subsidiary corporations. There is a large collection of corporate environmental disclosure research that exists; however, the majority of CED research that has been conducted focuses primarily on large multinational corporations, leaving a gap in understanding regarding how the CEDs of subsidiary operations align with those of their parent corporations (Kolk \& Pinkse, 2010; KPMG, 2011; Park \& 
Ghauri, 2015; Wilson, 2013). This will be addressed by analyzing the environmental disclosures from three categories of corporations: multinational parent companies, multinational subsidiary companies, and domestic Indian corporations. This will be done to explore if there has been a significant change in the disclosures of these corporations between 2002 and 2016 and if the environmental disclosures of the Indian subsidiary corporations are more similar to the domestic corporations or the parent corporations, in aspects of quality, quantity and diversity.

The history of CED research has witnessed an ever-increasing pattern of environmental disclosures for many corporations, primarily large corporations in developed countries (Ezhilarasi \& Kabra, 2017; Lin, 2008). Data available for this study will span from 2002 to 2016 . With this timeframe, it will be possible to identify certain changes and trends in environmental disclosure for corporations in all categories. It is expected that corporations operating in India will increase the quality, quantity, and diversity of environmental disclosures to meet government standards or surpass standards to maintain a competitive advantage. With that in mind, the following hypotheses are proposed:

H1a: Each corporation categories studied will increase the diversity of environmental disclosures over the timeframe examined.

H1b: Each corporation categories studied will increase the quantity of environmental disclosures over the timeframe examined.

H1c: Each corporation categories studied will increase the quality of environmental disclosures over the timeframe examined.

Previous research suggests that there are differences between environmental disclosures produced by corporations operating in developed and developing countries, with reasons for these differences including resources available, public scrutiny, and business culture (Ali et al., 2017; Adhikari et al., 2015; Luo et al., 2013). Many of these factors are altered depending on the ownership scheme of 
a corporation, foreign ownership is believed to have an impact on the level of disclosure from corporations operating in developing economies, although conflicting results fail to conclude if this is positive or negative (Chaklader \& Gulati, 2015; Haladu \& Salim, 2016; Hossain \& Andrew, 2006; Monteiro \& Aibar-Guzman, 2010; Zeng et al. 2011).

It is expected that the foreign subsidiary corporations will have environmental disclosures that are more similar to those of the domestic corporations rather than the parent multinational corporation. There are a number of reasons for this expectation. Previous research has shown that, in many cases across several countries and industries, that foreign ownership has little or negative impact on environmental disclosure (Chaklader \& Gulati, 2015; Darus et al., 2009; Haladu \& Salim, 2016). In these studies corporations operating in developing countries disclosed in a way that was similar to domestic corporations or even in a way that was poorer than domestic corporations despite the expectation that subsidiaries with foreign ownership would be better disclosures. There are some studies indicate that foreign ownership has a positive effect on environmental disclosure (Ezhilarasi \& Kabra, 2017; Riaz et al., 2015). Although a positive relationship is the less common finding in this track of research, many researchers indicate that a positive relationship is more expected and logically easier to explain. The purpose of examining this is to contribute to the examination of the effects of foreign ownership and add data from the analysis of the foreign owners in the form of parent multinationals to for additional context. Wilson (2013) found that the disclosures of foreign multinational subsidiaries were more similar to the disclosure of the local domestic corporations than to the parent corporation. This would indicate that the environmental disclosure of the subsidiary is localized, as opposed to standardized, within the multinational corporation.

The majority of previous CED research that has been conducted focuses primarily on large multinational corporations, such as the Fortune 500 companies or the top listed corporations in a selected country (KPMG, 2011; Wilson, 2013; Kolk \& Pinkse, 2010). Studies examining the CEDs of 
multinationals rarely include subsidiary corporations, and studies focused on domestic corporations rarely make a distinction between domestically owned corporations and foreign-operated multinational subsidiaries (Wilson, 2013). Empirical literature reviews have called for further research in the area of CED and more comprehensive analysis and still fail to identify the gap of subsidiary companies (Ali et al., 2017; Fifka, 2013). The objective of this research is to analyze CEDs from primary multinational corporations, multinational subsidiary corporations and domestic corporations to determine the quality, quantity, and diversity of information presented to determine if multinational corporations are standardizing CEDs in all areas of operation, in this case in developed and developing countries of operation. The idea of CED standardization is based on the standardization as utilized in international marketing (Haron, 2016 ;), which has already been integrated and applied to the analysis of corporations reporting and disclosure practices (Arora \& Lodhia, 2017; Cho \& Patten, 2007; Hunter \& Bansal, 2006).

Building on the discussion above, the following hypotheses are proposed:

H2a: The diversity of environmental disclosures of P-MNCs and I-MNCs will lack standardization, and the environmental disclosures of I-MNCs will be more similar to those of domestic corporations than the P-MNC.

H2b: The quantity of environmental disclosures of P-MNCs and I-MNCs will lack standardization, and the environmental disclosures of I-MNCs will be more similar to those of domestic corporations than the PMNC.

H2c: The quality of environmental disclosures of P-MNCs and I-MNCs will lack standardization, and the environmental disclosures of I-MNCs will be more similar to those of domestic corporations than the PMNC. 


\subsection{Methods}

\subsubsection{Sample Selection}

Selection of the multinational corporations was determined by first compiling a list of agrochemical corporations; in total, over 200 companies were initially included. From there, the twenty listed corporations with the highest revenue were selected. From this list of twenty, corporations were eliminated if a corporation's headquarter operations were not based in North America or Europe or if the primary business operations were not in the agrochemical industry. The reason North America and Europe were focused on is that companies based in these locations consistently report in English. Additionally, if a corporation did not have a subsidiary corporation operating in India it was also eliminated. There were nineteen corporations from the original list of twenty that met the selection criteria (i.e. only one company was eliminated from the list after applying the screening criteria). From the list of the remaining 19 companies, four corporations were randomly selected and consequently selected to be included in the study. The reason four corporations were selected is that the manual coding of documents is highly time and labour intensive, meaning the sample size had to be limited, particularly given that the timeframe of the analysis covers 15 years of disclosures. These corporations were selected as the P-MNC corporations [Table 1]: Bayer CropScience, Syngenta AG, BASF SE, and Monsanto Corporation. The Indian subsidiary corporations were selected correspondingly to the parent multinational corporations. These corporations operate under the names Bayer CropScience (India), Syngenta India Ltd., BASF India Ltd., and Monsanto India Ltd.

The Domestic Indian corporations were selected by compiling a list of Indian agrochemical companies, which resulted in a sample of fifty-four corporations. This was narrowed by eliminating non-publicly traded corporations and corporations that did not have primary business operations in agrochemicals. This resulted in twenty-six appropriate corporations, from which four companies were randomly chosen. 
The final selection of corporations was United Phosphorus Ltd., Zuari Agro Chemical Ltd., Rallis Ltd., and Coromandel International Ltd. In total twelve corporations were selected for this study and represent three different categories of corporations, P-MNC, I-MNC, and domestic corporations.

\subsubsection{Content Analysis}

This study utilizes content analysis to quantify the qualitative and quantitative information published by the selected corporations. Content analysis is a common tool used to systematically analyze reports and written material (Beck et al., 2010). By analyzing the content of the material, manageable quantitative data can be extracted (Weber, 1990). Content analysis can range from very simple analysis, where a single keyword is quantified from the text to more complex analysis measuring multiple themes (Weber, 1990). Furthermore, content analysis can be coded either by the user themselves or by a computer Weber, 1990). Computer-based analysis results in excellent reliability but cannot be applied to all coding requirements.

Table 1: Profiles of corporations selected for research

\begin{tabular}{|c|c|c|c|c|}
\hline Category & Corporation & Location & Revenue for FY16 & $\begin{array}{l}\text { Number of } \\
\text { Employees for } \\
\text { FY16 }\end{array}$ \\
\hline \multirow[t]{4}{*}{$\begin{array}{l}\text { Parent } \\
\text { Multinational }\end{array}$} & $\begin{array}{l}\text { Monsanto } \\
\text { Corporation }\end{array}$ & USA & 13,502 & 20,800 \\
\hline & BASF SE & Germany & 57,550 & 113,830 \\
\hline & $\begin{array}{l}\text { Bayer Cropscience } \\
\text { AG }\end{array}$ & Germany & 46,769 & 115,200 \\
\hline & Syngenta AG & Switzerland & 12,790 & 27,810 \\
\hline \multirow[t]{3}{*}{$\begin{array}{l}\text { Subsidiary } \\
\text { Multinational }\end{array}$} & $\begin{array}{l}\text { Monsanto India } \\
\text { Ltd }\end{array}$ & India & 123.7 & 268 \\
\hline & BASF India Ltd & India & $10,232.94$ & 1,868 \\
\hline & Bayer Cropscience & India & 5460.00 & 1,126 \\
\hline
\end{tabular}




\begin{tabular}{|l|l|l|l|l|}
\hline \multirow{2}{*}{ Domestic } & Ltd (India) & & & \\
\cline { 2 - 5 } & Syngenta India Ltd & India & 440.56 & Not disclosed \\
\hline & Rallis & India & 330.18 & 952 \\
\cline { 2 - 5 } & Coromandel & India & $2,164.81$ & 4,309 \\
\cline { 2 - 5 } & Zuari & India & 728.24 & 727 \\
\cline { 2 - 5 } & $\begin{array}{l}\text { United } \\
\text { Phosphorus }\end{array}$ & India & 3141.11 & 3,489 \\
\hline
\end{tabular}

Beck et al. (2010) classify content analysis into two approaches: mechanistic or interpretive. Mechanistic approaches are the more traditional content analysis, where written work is analyzed based on the frequency of keywords, or simply by the quantity of the report (Beck et al., 2010). A mechanistic approach to content analysis is simple and commonly used but can lack value due to the inability to contextualize the information. An interpretive approach is meant to acknowledge the narrative of the written report by focusing on the message (Beck et al., 2010). An interpretive approach provides more complete insight into the data collected but is more complex to perform and not as commonly used.

The content analysis approach selected for this research is consolidated narrative interrogation (CONI). Developed by Beck et al. (2010), it is specifically tailored for the analysis of environmental information. Consolidated narrative interrogation (CONI) attempts to create a hybrid of mechanistic and interpretive approaches to develop a content analysis that is both user-friendly and comprehensive (Beck et al., 2010). CONI was selected for use in this research because it provides a more comprehensive analysis of the data when compared to selecting an analysis that is exclusively mechanistic or interpretive. This research analyses data collected by two coders to ensure the reliability of the data, inter- and intra- user reliability testing was performed on a 10 percent sample of documents that were subjected to a testretest analysis. Intra-user reliability was rated at 0.91 and inter-user reliability was rated at 0.85 using 
Krippendorf's alpha. Krippendorf's alpha is considered conservative, and a score above 0.80 is viewed as widely acceptable (De Swert, 2012; Tilt, 2001).

CONI is unique because it allows the user to rate a unit, in this case, a sentence, on three criteria at once. CONI measures the diversity, quality and quantity of a document by recording the category of disclosure, the quality of the information, and the overall amount of relevant information. The unit for measurement in this method is a sentence; therefore, any sentence containing relevant corporate environmental disclosure (CED) is analyzed according to the following steps.

\subsubsection{Diversity Coding}

The first step in the process is to determine the diversity category best suited to the relevant sentence. Beck et al. (2010) initially developed 12 Categories and 48 sub-categories for diversity coding. Beck et al. (2010) used the measurement of diversity to assess the environmental message, the more categories that a CED reports on the more comprehensive the publication. This study, however, will use the adapted categories developed by Wilson (2013), which features 18 categories and 59 sub-categories [Table 2]. Wilson developed these additional categories to better suit the application of the method specifically to the disclosure material of agrochemical companies and to address environmental issues that had come to light following 2010 when the categories were developed. The categories added where tested to ensure that they were reliable and mutually exclusive (Wilson, 2013). Newly added categories were GEN-8, PROD-1, PROD-2, SUST-4, ACT-5, RC, ENE-3, INP-1, INP-2, SUPP, COMP-1, COMP-2, COMP3, and ECE. Additionally, some categories that were present in Beck et al.'s original categories were removed in order to be more specifically addressed in the new categories, these were by the original classification given in Beck et al., POLL-5, POLL-6 (Wilson, 2013).

All sentences containing CED information are classified into a category and sub-category based on the overall meaning of the sentence. If the coder is unable to determine a singular meaning for the 
sentence, then the sentence will be classified based into the category relevant to the first point made in the sentence.

To score the diversity of a publication, categories that are reported on are assigned a value of one and categories that are not reported on are given a value of zero. These values are totalled to give the final score. The highest score possible for a publication to receive is 18 .

\subsubsection{Quality Coding}

After a unit has been classified into a diversity category, it is then coded based on the quality of information disclosed in the sentence. This measurement is used to determine the "the level of information provided combined with the depth or detail of the disclosure" (Beck et al., 2010, pg. 213). This step includes five "types" of disclosures based on whether the information present is high quality

Table 2: Diversity coding categories and sub-categories for CONI diversity analysis

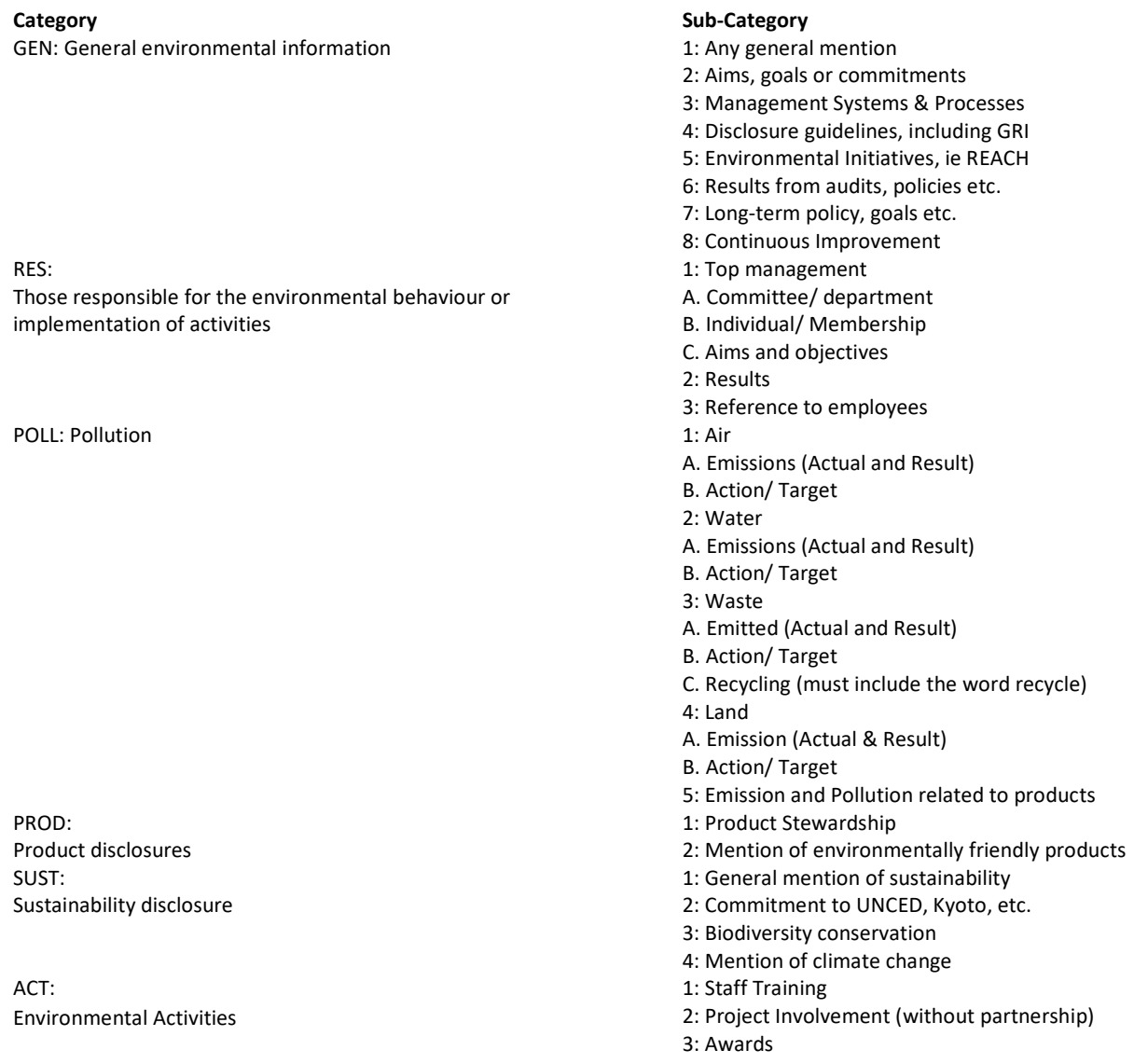


identifying the quality of disclosure in a sentence is not difficult based on the specific requirements of each category.

\subsubsection{Quantity Coding}

The final step of the coding process is to determine the quantity of CED sentences reported by the corporations. This is measured by adding the total number of sentences containing information pertaining to the environment as described by the 18 categories. Adding the total number of these sentences will reveal the total quantity for the entire report.

\subsubsection{Analysis}

The analysis was performed separately on measurements of diversity, quality, and quantity data to preserve the multifaceted advantage of using the CONI method. The analysis focused on two main elements. First, a simple linear regression was used to indicate the significance of the change in a parameter over the timeframe. Second, a repeated measures two-way ANOVA was used to assess the

Table 3: Examples of Quality type disclosure classifications

\begin{tabular}{|l|l|l|}
\hline Disclosure Type & Example & Explanation \\
\hline Type 1 & $\begin{array}{l}\text { "Most importantly, we approach } \\
\text { sustainability as a long-term, collaborative } \\
\text { effort." (Monsanto CSR Report, 2016 pg. 4) }\end{array}$ & $\begin{array}{l}\text { This sentence is vague and includes } \\
\text { no goal or plan for execution; } \\
\text { because of this it offers little value } \\
\text { and is classified as a Type 1. }\end{array}$ \\
\hline Type 2 & $\begin{array}{l}\text { "In November 2015, Monsanto signed the } \\
\text { White House American Business Act on } \\
\text { Climate Pledge, which affirmed our } \\
\text { commitment to climate action and our } \\
\text { support for a strong outcome from the } \\
\text { United Nations Framework Convention on } \\
\text { Climate Change Conference in Paris } \\
\text { (COP21) that took place in December } \\
\text { 2015." (Monsanto CSR Report, 2015, pg. } \\
\text { 53) }\end{array}$ & $\begin{array}{l}\text { This disclosure offers information } \\
\text { beyond a general level, including a } \\
\text { specific commitment to and named } \\
\text { authority. Note the use of the year } \\
\text { does not count as quantitative } \\
\text { information and therefore does not } \\
\text { make this disclosure appropriate for } \\
\text { Type 4 classification; it is instead } \\
\text { classified as a Type 2. }\end{array}$ \\
\hline
\end{tabular}




\begin{tabular}{|l|l|l|}
\hline Type 3 & $\begin{array}{l}\text { "The Group's total energy consumption } \\
\text { meanwhile was even down 2.8\% at 80.8 } \\
\text { petajoules." (Bayer Annual report, 2013, } \\
\text { pg. 132" }\end{array}$ & $\begin{array}{l}\text { This disclosure offers primarily } \\
\text { quantitative information with little } \\
\text { narration or explanation. }\end{array}$ \\
\hline Type 4 & $\begin{array}{l}\text { "Additionally, during the current year, the } \\
\text { Company has recognized the business } \\
\text { interruption claim amounting to '2500 } \\
\text { lakhs received from the Insurance company } \\
\text { on account of the 'Hudhud' cyclone in } \\
\text { Vishakapatnam unit." (Coromandel Annual } \\
\text { Report, 2015, pg 123) }\end{array}$ & $\begin{array}{l}\text { This sentence includes an element of } \\
\text { both quantitative and qualitative } \\
\text { information, but doesn't compare to } \\
\text { any additional years; for this reason, } \\
\text { it is classified as a Type 4 disclosure. }\end{array}$ \\
\hline $\begin{array}{l}\text { [Chart format] "Total waste; Landfill; FY14 } \\
\text { 8,600; FY15 7,060" (Monsanto CSR Report, } \\
2015, \text { pg 76) }\end{array}$ & $\begin{array}{l}\text { This chart entry contains data for the } \\
\text { financial year 2014 and 2015; } \\
\text { because a Type } 5 \text { disclosure doesn't } \\
\text { require a narrative component, this } \\
\text { classifies as a Type 5. }\end{array}$ \\
\hline
\end{tabular}

significance of the difference in reporting between corporation categories and the interaction over time.

Using a repeated measures analysis helps account for individual variability of a corporation and the possibility that a corporation may be an exceptionally good or poor reporter.

\subsection{Results and Discussion}

Results were collected from a sample of 12 companies from 2002 to 2016 in three different categories of corporations: domestic, I-MNC, and P-MNC.

\subsubsection{Significance Over Time}

One of the objectives of this research was to examine the trends of disclosures from the three categories over a fifteen-year timeframe. To do this, simple linear regression was performed for the results from each corporation for diversity, quantity, and quality to determine if there was statistically significant change over time. 


\subsubsection{Diversity}

All corporations in all three categories showed an increase in diversity over time [Fig 1-3]. In 2002, the average diversity score for domestic corporations, I-MNC, and P-MNC was 10, 9.25, and 11, respectively. By 2016 , the mean diversity scores had increased to $13.5,14.25$ and 16.75 for domestic corporations, IMNC, and P-MNC, respectively. Plotting the mean diversity for each category reveals that the rate of increase for domestic and I-MNC corporations is very similar, while the rate of increase for P-MNC is higher [Fig 4].

Results show that the change in diversity over time was significant for half of the domestic corporations (UPL $(p=0.01466)$, Zuari $(p=0.001083))$, half of the I-MNCs (I-BASF $(p=0.027049)$, I-Bayer $(p=0.069676$ )), and all P-MNC (P-BASF ( $p=0.006416)$, P-Bayer ( $p=0.002247)$, P-Monsanto ( $p=0.000169)$, P-Syngenta $(p=0.093532))$. Overall regression based on the mean value of each category shows statistical significance for domestic corporations $(p=0.002728)$ and P-MNC $(p=2.67 E-05)$, but not for I-MNC $(p=$ 0.173379). Regardless, it is observable from the data that the diversity scores of I-MNCs are increasing overall despite the lack of significance [Fig 2]. Plotting the mean diversity for each category also reveals that the rate of increase for domestic and I-MNC corporations is very similar, while the rate of increase for P-MNC is higher [Fig 4].

By the end of 2016, several of the P-MNCs were reporting on all possible categories of disclosure, but IMNCs and domestic corporations noticeably lacked or had low disclosure in areas such as environmental responsibility/management (RES), pressure groups (PRESS), information retrieval process (IRP), responsible care $(\mathrm{RC})$ and pollution (POLL). As previously discussed, legitimacy is a key reason for disclosing information deemed desirable by societal pressures and this observation may be explained by the application of legitimacy theory. I-MNCs and domestic corporations both operate in India which means they both share some common stakeholders (local community, customers, local government) 
that P-MNCs do not. If I-MNCs and domestic corporations perceive a lack of interest in certain areas by the stakeholders in the societies in which they operate, they may choose not to disclose on these topics. Additionally, I-MNCs and domestic corporations may avoid disclosing information pertaining to these areas, if they fear losing legitimacy based on poor or inadequate performance.

\subsubsection{Quantity}

Similar trends are evident for the analysis of quantity [Fig 5-8]. In 2002, the average quantity for domestic corporations was 69.5, for I-MNC was 50, and for P-MNC was 230.25 . By 2016, the average quantity of CED material published was $233.5,128.25$ and 577, for domestic corporations, I-MNC and PMNC, respectively. For quantity measurements, P-MNCs scored much higher than domestic and IMNCs. CEDs from domestic corporations on average were slightly higher than I-MNCs [8].

Quantity over time is a statistically significant factor for half of the domestic corporations (UPL ( $p=$ 0.002953), Zuari $(p=0.003492))$, one I-MNC (I-Syngenta $(p=0.097297))$, and three P-MNC (P-BASF $(p=0.012364)$, P-Monsanto $(p=3.94919 E-05), P-S y n g e n t a(p=0.000613))$. Based on mean quantity, time is a significant factor for all categories of corporation (domestic $(p=0.001097), I-M N C(p=0.049118), P-M N C$ $(p=0.00044))$.

Outliers in data, including a cluster of reports from Rallis India for the years 2007, 2008, 2009, UPL for the year 2016, I-BASF for the year 2010 and P-Bayer CropScience years 2004 and 2012. Rallis India experienced an increase in profits following a period of losses in the years leading up to the publication of the highlighted reports, also a new executive director came into power in 2007. UPL didn't have outstanding financial growth leading up to 2016, however, the 2016 annual report titled "Doing Things Better" and highlighted the renewed importance of CSR performance. In 2010 I-BASF faced a large merger between Ciba India Ltd and BASF India, which also increase company sales. No significant events connected to Bayer CropScience could be found around the year 2004, however around 2012 scientific 
articles (Henry et al., 2012; Whitehorn et al., 2012) and news articles (Borenstein, 2012; Marcott, 2012) reporting on the effects of neonicotinoid pesticides on bumblebees received significant attention. Bayer CropScience was particularly villanized as they are leading producers of this class of pesticides. While these events cannot be exclusively used to explain the increase in the quantity of the reports published by these companies on specific years, it does suggest that corporations may vary their disclosure in response to other business and reputational influences.

As predicted by previous research, P-MNCs do show a significantly higher quantity of CEDs (Ezhilarasi \& Kabra, 2017; KPMG, 2011). Additionally, by examining the quantity over so many years, it can also be seen that the rate of increase is higher for P-MNC than for domestic corporations and I-MNC [Fig 8]. Therefore, despite that fact that all the corporate categories have increased quantity over the last 
Table 4: Diversity scores for CEDs from domestic corporations (Coromandel, Rallis India Ltd, United Phosphorus and Zuari), I-MNC (I-BASF, I-Bayer Cropscience, I-Monsanto, I-Syngenta) and P-MNC (P-BASF, P-Bayer Cropscience, P-Monsanto and P-Syngenta) for the years $2002-2016$.

\begin{tabular}{|c|c|c|c|c|c|c|c|c|c|c|c|c|c|c|c|}
\hline & 2002 & 2003 & 2004 & 2005 & 2006 & 2007 & 2008 & 2009 & 2010 & 2011 & 2012 & 2013 & 2014 & 2015 & 2016 \\
\hline Coromandel & 12 & 12 & 12 & 7 & 8 & 8 & 7 & 9 & 12 & 11 & 8 & 12 & 9 & 11 & 13 \\
\hline $\begin{array}{l}\text { Rallis India } \\
\text { Ltd }\end{array}$ & 12 & 10 & 10 & 10 & 10 & 16 & 16 & 16 & 11 & 12 & 10 & 12 & 11 & 12 & 14 \\
\hline $\begin{array}{l}\text { United } \\
\text { Phosphorus } \\
\text { Ltd (UPL) }\end{array}$ & 9 & 10 & 12 & 11 & 11 & 11 & 13 & 10 & 13 & 12 & 12 & 11 & 14 & 11 & 14 \\
\hline Zuari & 7 & 7 & 6 & 6 & 6 & 6 & 7 & 8 & 8 & 9 & 9 & 6 & 10 & 14 & 13 \\
\hline I-BASF & 11 & 11 & 10 & 12 & 10 & 10 & 9 & 10 & 15 & 16 & 13 & 13 & 10 & 14 & 16 \\
\hline $\begin{array}{l}\text { I-Bayer } \\
\text { Cropscience }\end{array}$ & 6 & 11 & 12 & 12 & 11 & 12 & 12 & 13 & 14 & 12 & 11 & 11 & 11 & 11 & 16 \\
\hline I-Monsanto & 11 & 12 & 12 & 11 & 8 & 8 & 10 & 11 & 13 & 8 & 10 & 13 & $\mathrm{~N} / \mathrm{A}$ & 14 & 15 \\
\hline I-Syngenta & 9 & 8 & 11 & 8 & N/A & $\mathrm{N} / \mathrm{A}$ & 9 & 11 & 9 & 10 & $\mathrm{~N} / \mathrm{A}$ & 10 & 11 & 10 & 10 \\
\hline P-BASF & 17 & 16 & 15 & 17 & 16 & 16 & 17 & 18 & 17 & 17 & 18 & 17 & 17 & 18 & 18 \\
\hline $\begin{array}{l}\text { P-Bayer } \\
\text { Cropscience }\end{array}$ & 6 & 7 & 16 & 15 & 16 & 17 & 18 & 16 & 17 & 17 & 18 & 17 & 17 & 18 & 18 \\
\hline P-Monsanto & 7 & 9 & 10 & 15 & 14 & 11 & 15 & 14 & 14 & 15 & 16 & 15 & 16 & 16 & 16 \\
\hline P-Syngenta & 14 & 13 & 10 & 14 & 15 & 15 & 12 & 14 & 14 & 13 & 13 & 15 & 15 & 15 & 15 \\
\hline
\end{tabular}

N/A represents a lack of disclosure 


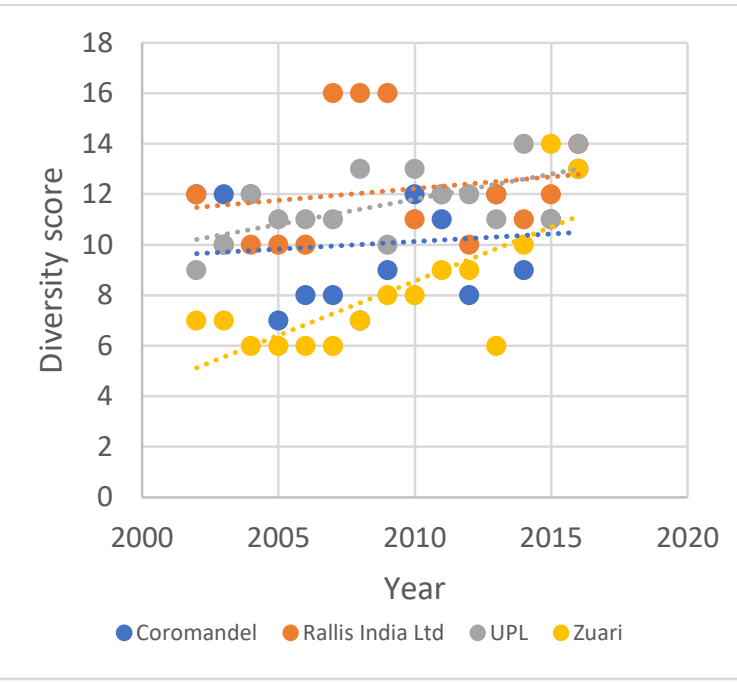

Figure 1. Total diversity score of disclosure for domestic Indian corporations from 2002-2016. Coromandel $(p=0.649221)$, Rallis $(p=0.518678)$, UPL ( $p=0.01466)$, Zuari $(p=0.001083)$

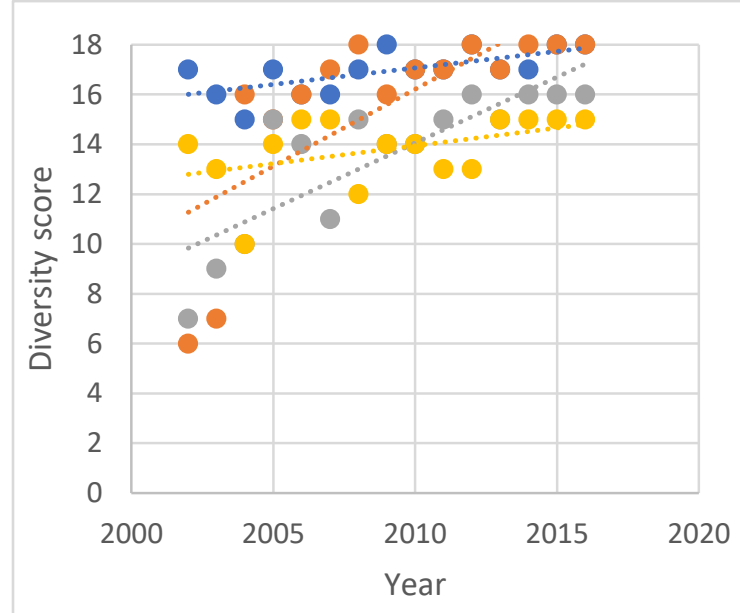

Figure 3. Total diversity score of disclosures from 2002-2016. $P$-BASF $(p=0.006416), P$-Bayer $(p=0.002247), P$-Monsanto $(p=0.000169), P$ Syngenta $(p=0.093532)$

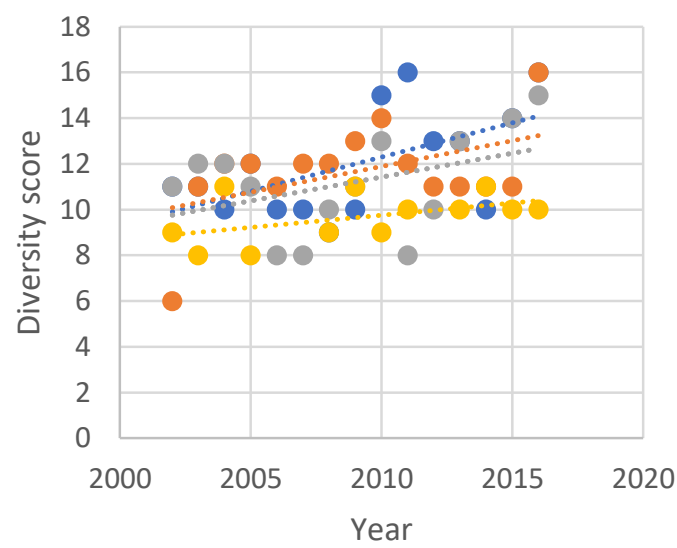

I-BASF I-Bayer CropScience I-Monsanto I-Syngenta

Figure 2. Total diversity score of disclosure for 1MNCs from 2002-2016. I-BASF ( $p=0.027049)$, IBayer $(p=0.069676)$, I-Monsanto $(p=0.16152)$, ISyngenta $(p=0.110613)$

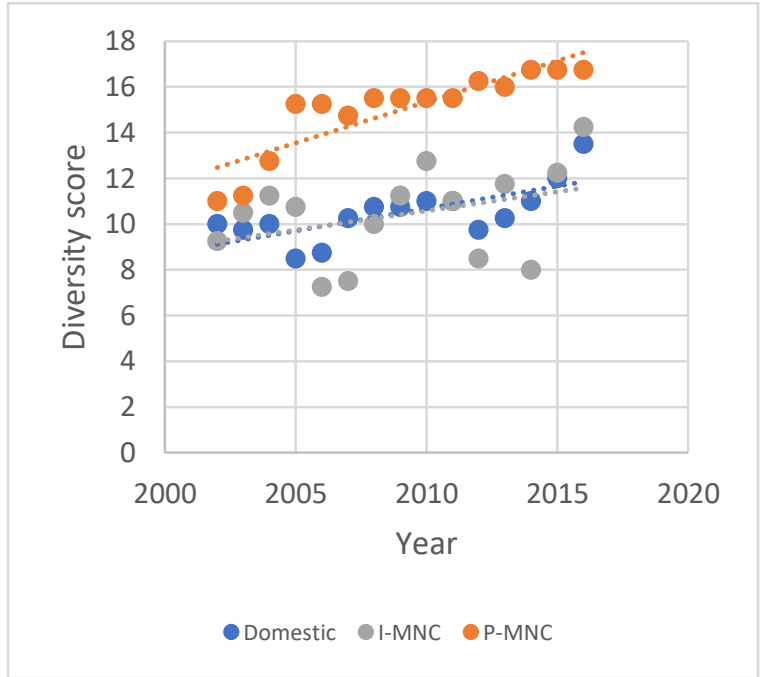

Figure 4. Mean diversity score of disclosures from domestic Indian corporations, I-MNCs and PMNCs from 2002-2016. Domestic $(p=0.002728)$, I-MNC $(p=0.173379), P-M N C(p=2.67 E-05)$ 
Table 5: Quantity of CEDs from domestic corporations (Coromandel, Rallis India Ltd, United Phosphorus and Zuari), I-MNC (I-BASF, I-Bayer

\begin{tabular}{|l|l|l|l|l|l|l|l|l|l|l|l|l|l|l|l|}
\hline & 2002 & 2003 & 2004 & 2005 & 2006 & 2007 & 2008 & 2009 & 2010 & 2011 & 2012 & 2013 & 2014 & 2015 & 2016 \\
\hline
\end{tabular}

Cropscience, I-Monsanto, I-Syngenta) and P-MNC (P-BASF, P-Bayer Cropscience, P-Monsanto and P-Syngenta) for the years $2002-2016$. 


\begin{tabular}{|c|c|c|c|c|c|c|c|c|c|c|c|c|c|c|c|}
\hline Coromandel & 101 & 106 & 88 & 48 & 40 & 46 & 56 & 60 & 101 & 90 & 53 & 79 & 76 & 95 & 192 \\
\hline $\begin{array}{l}\text { Rallis India } \\
\text { Ltd }\end{array}$ & 89 & 61 & 58 & 55 & 54 & 275 & 264 & 344 & 89 & 94 & 104 & 140 & 88 & 175 & 205 \\
\hline $\begin{array}{l}\text { United } \\
\text { Phosphorus } \\
\text { Ltd (UPL) }\end{array}$ & 22 & 60 & 67 & 74 & 83 & 78 & 80 & 80 & 106 & 95 & 131 & 131 & 143 & 104 & 372 \\
\hline Zuari & 66 & 63 & 54 & 72 & 49 & 53 & 52 & 56 & 67 & 58 & 189 & 59 & 185 & 202 & 165 \\
\hline I-BASF & 60 & 73 & 68 & 72 & 64 & 73 & 96 & 97 & 103 & 64 & 107 & 81 & 71 & 91 & 163 \\
\hline $\begin{array}{l}\text { I-Bayer } \\
\text { Cropscience }\end{array}$ & 48 & 53 & 93 & 106 & 75 & 79 & 94 & 91 & 100 & 85 & 85 & 64 & 72 & 57 & 149 \\
\hline I-Monsanto & 54 & 67 & 73 & 63 & 53 & 44 & 65 & 179 & 129 & 71 & 76 & 97 & - & 111 & 141 \\
\hline I-Syngenta & 38 & 38 & 38 & 33 & - & - & 34 & 45 & 42 & 50 & - & 80 & 47 & 63 & 60 \\
\hline P-BASF & 589 & 299 & 364 & 393 & 463 & 433 & 638 & 575 & 552 & 535 & 517 & 557 & 580 & 590 & 615 \\
\hline $\begin{array}{l}\text { P-Bayer } \\
\text { Cropscience }\end{array}$ & 43 & 40 & 1141 & 743 & 735 & 857 & 1046 & 903 & 900 & 897 & 1282 & 739 & 757 & 692 & 599 \\
\hline P-Monsanto & 29 & 76 & 106 & 282 & 318 & 398 & 91 & 376 & 398 & 607 & 519 & 278 & 620 & 649 & 701 \\
\hline P-Syngenta & 260 & 147 & 165 & 214 & 258 & 339 & 258 & 273 & 277 & 258 & 265 & 284 & 341 & 398 & 393 \\
\hline
\end{tabular}




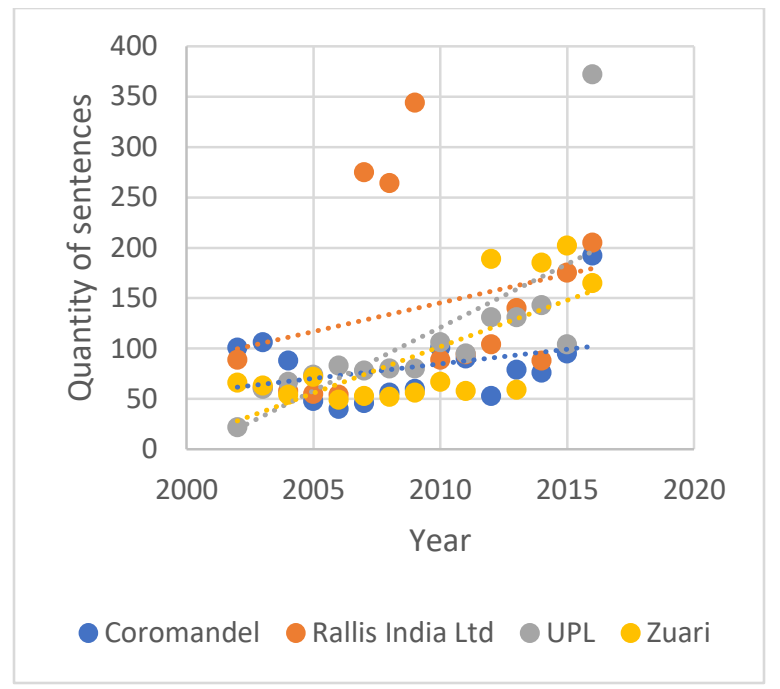

Figure 5. Total quantity of disclosures for domestic Indian corporations from 2002-2016. Coromandel $(p=0.212673)$, Rallis $(p=0.318744)$, UPL $(p=$ $0.002953)$, Zuari ( $p=0.003492)$.

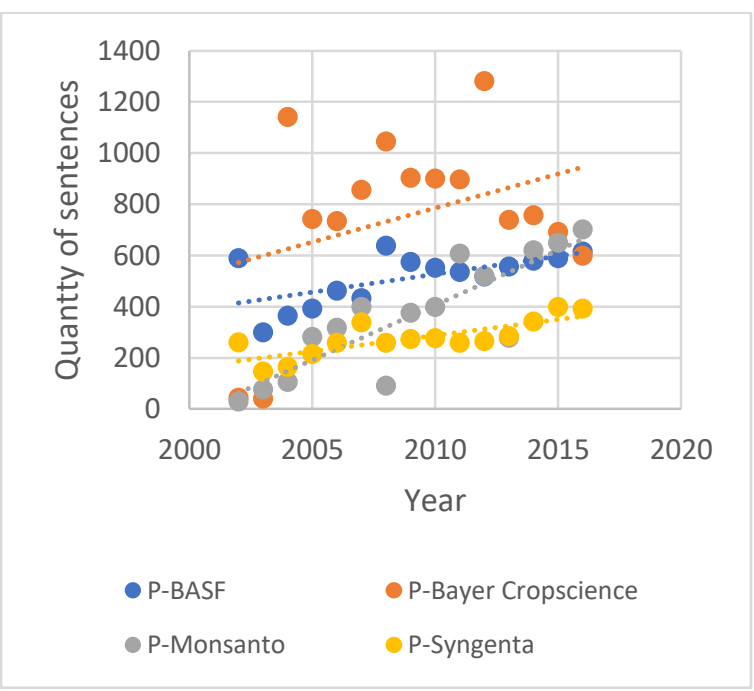

Figure 7. Total quantity of disclosure for P-MNCS from 2002-2016. $P$-BASF $(p=0.012364), P$-Bayer $(p=0.203456), P-M o n s a n t o(p=3.94919 E-05), P$ Syngenta $(p=0.000613)$.

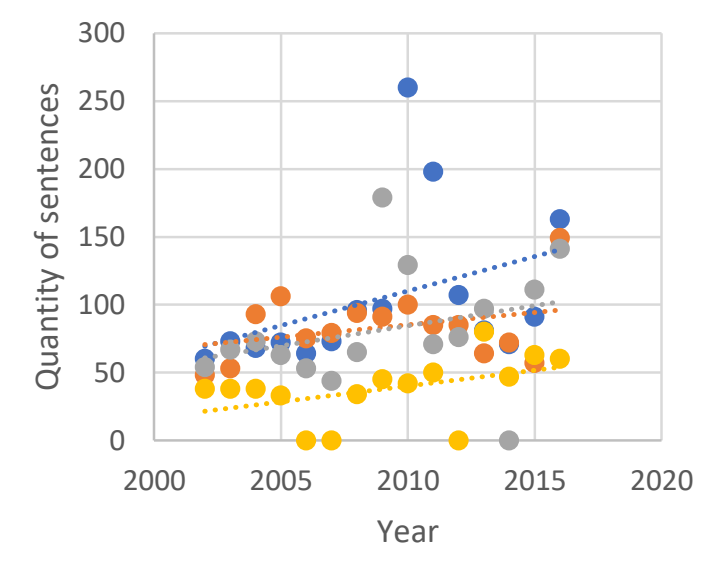

- I-BASF - I-Bayer Cropscience I-Monsanto

Figure 6. Total quantity of disclosure for I-MNCS from 2002-2016. I-BASF ( $p=0.144962)$, I-Bayer $(p=0.242071), I-$ Monsanto $(p=0.274214), I-$ Syngenta $(p=0.097297)$.

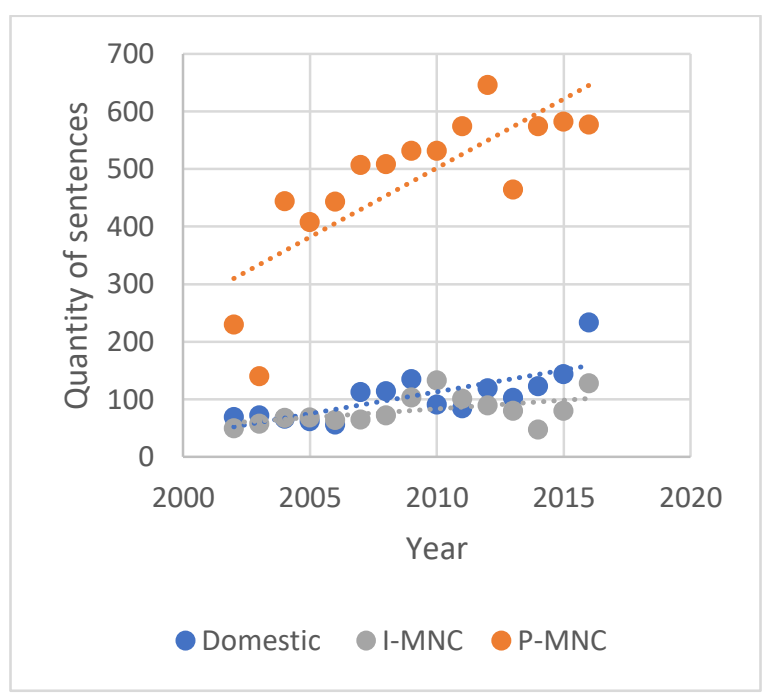

Figure 8. Mean quantity of disclosure for domestic Indian corporations, I-MNCs and PMNC for 2002-2016. Domestic $(p=0.001097), ~ I-$ MNC $(p=0.049118), P-M N C(p=0.00044)$.

fifteen years, there continues to be a gap between the quantity of disclosure from P-MNC and from the other corporations. In fact, because the rate of increase in quantity from P-MNCs is so high, the difference between the quantity of disclosure for P-MNC and Indian based corporations is becoming 
greater over time.

\subsubsection{Quality}

Quality of disclosures was determined by the type of disclosure from Type 1 to Type 5. It was measured by the percent of disclosures sorted by type. Domestic corporations [Fig 9-12] had considerable Type 5 disclosures around the 2004-2007 time period caused by disclosures from Rallis Indian Ltd, but fewer by 2016, with the exception of Zuari [Fig 12]. I-MNCs had large portions of Type 1 disclosures and fewer Type 2 disclosures than domestic corporations [Fig 13-16]. P-MNC had the most uniform and consistent portions of Type 1 and 2 disclosures, but overall low portions of high-quality disclosures (Type 4 and 5). Results show that the change in quality over time is significant for at least one of the types of quality for all domestic corporations, two I-MNCs (I-BASF and I-Bayer), and all P-MNCs.

General trends showed a decrease in the total percentage of Type 1 and Type 5 disclosures for domestic corporations, as well an increase in Type 2 and 4 disclosures. It is encouraging to see domestic corporations moving away from Type 1 disclosures as the foundation of their CEDs, given that they do not offer much useful information. Domestic corporations also had a high level of Type 5 disclosure compared to other corporations in the earlier years, indicating a high level of quality. The percentage of Type 5 disclosures for most domestic corporations declined around 2007. Also around this time, the quantity of CED from domestic corporations increased, this could be related to the decline in the percentage of Type 5 disclosures. It may also be worthy to note that the portion of Type 5 disclosures appeared to decline further after 2013, which was the year the Indian government released the updated Companies Act that outlined the required implementation of CSR reporting. Results are insufficient to suggest if there is a relationship between the two. However, previous research on the topic showed that in China mandatory environmental disclosure improved quality (Wang et al. 2017), meanwhile Doedhar 
(2016) predicted that India's mandatory CSR may reduce the quality or related initiatives, this is a potential area for future research.

Trends among the quality of CEDs from I-MNC show that Type 4 and 5 disclosures decreased over the timeframe studied, indicating a decline in high-quality disclosures. Meanwhile, the percentage of Type 1 disclosures increased slightly. The decrease in both high-level quality disclosures suggests that the bulk of information that has been added as the quantity of CED has increased is of a low quality, thus bringing down the overall percentage of high-quality information.

Results from P-MNC show that while P-MNCs have experienced that largest growth in the quantity of all the corporations studied, the quality of disclosures remains the most consistent. This indicated that, while there was an increase in the amount of information published, P-MNC corporations are balancing the amount of high- and low-quality disclosures and not simply adding low-quality disclosures for the sake of added quantity.

\subsubsection{Significance of Standardization}

In order to determine the level of standardization of CED reporting across the three corporate categories, a repeated measures ANOVA analysis was performed to examine the significance between publication year and corporation category for each parameter. Using a repeated measures analysis accounts for an individual corporation's change over time, while analyzing the significance of the corporate category. This is done to account for the lack of independence between these two variables. From the results, it is possible to observe if there is a statistically significant interaction between the publication year and the category of the corporation, as well as if the publication year or corporation category independently have a significant effect on the parameter measured. 
Table 6. Quality type by percent for CEDs from domestic corporations (Coromandel, Rallis India Ltd, United Phosphorus and Zuari), I-MNC (I-BASF, IBayer Cropscience, I-Monsanto, I-Syngenta) and P-MNC (P-BASF, P-Bayer Cropscience, P-Monsanto and P-Syngenta) for the years $2002-2016$.

\begin{tabular}{|c|c|c|c|c|c|c|c|c|c|c|c|c|c|c|c|c|}
\hline Corporation & Type & 2002 & 2003 & 2004 & 2005 & 2006 & 2007 & 2008 & 2009 & 2010 & 2011 & 2012 & 2013 & 2014 & 2015 & 2016 \\
\hline \multirow[t]{5}{*}{ Coromandel } & 1 & 48.51 & 44.34 & 39.33 & 43.75 & 37.50 & 36.96 & 46.43 & 40.00 & 44.55 & 44.44 & 13.21 & 22.78 & 36.84 & 14.13 & 22.39 \\
\hline & 2 & 23.76 & 34.91 & 39.33 & 18.75 & 17.50 & 8.69 & 8.93 & 20.00 & 26.73 & 23.33 & 45.28 & 43.04 & 51.32 & 68.48 & 52.60 \\
\hline & 3 & 9.91 & 5.66 & 3.37 & 4.17 & 2.50 & 4.35 & 1.79 & 8.33 & 7.92 & 7.78 & 7.54 & 30.38 & 6.58 & 3.26 & 10.94 \\
\hline & 4 & 3.96 & 3.77 & 1.12 & 2.08 & 5.00 & 17.39 & 8.93 & 0.00 & 1.98 & 1.11 & 3.77 & 3.80 & 5.26 & 14.13 & 12.50 \\
\hline & 5 & 13.86 & 11.32 & 16.85 & 31.25 & 37.50 & 32.61 & 33.93 & 31.67 & 18.81 & 23.33 & 30.79 & 0.00 & 0.00 & 0.00 & 1.56 \\
\hline \multirow[t]{5}{*}{ Rallis India Ltd } & 1 & 46.07 & 42.62 & 32.76 & 34.54 & 27.78 & 27.64 & 30.68 & 33.14 & 41.57 & 42.55 & 34.62 & 26.43 & 53.41 & 23.43 & 29.27 \\
\hline & 2 & 7.86 & 18.03 & 31.03 & 29.09 & 35.18 & 52.00 & 46.21 & 46.80 & 29.21 & 28.72 & 41.35 & 52.14 & 40.91 & 51.43 & 53.66 \\
\hline & 3 & 6.74 & 3.28 & 3.45 & 1.82 & 1.85 & 2.18 & 2.65 & 2.03 & 0.00 & 3.19 & 0.00 & 0.00 & 0.00 & 0.00 & 0.00 \\
\hline & 4 & 0.00 & 1.64 & 0.00 & 0.00 & 0.00 & 0.00 & 0.76 & 1.74 & 1.12 & 2.12 & 1.92 & 5.00 & 3.41 & 24.00 & 15.12 \\
\hline & 5 & 39.33 & 34.43 & 32.76 & 34.54 & 35.18 & 18.18 & 19.70 & 16.28 & 28.09 & 23.40 & 22.11 & 16.43 & 2.27 & 1.14 & 1.95 \\
\hline \multirow{5}{*}{$\begin{array}{l}\text { United } \\
\text { Phosphorus } \\
\text { Ltd (UPL) }\end{array}$} & 1 & 77.27 & 61.67 & 41.79 & 28.38 & 36.14 & 35.89 & 40.00 & 33.75 & 35.85 & 36.84 & 62.59 & 48.09 & 63.64 & 50.96 & 54.30 \\
\hline & 2 & 18.18 & 23.33 & 29.85 & 41.89 & 30.12 & 32.05 & 26.25 & 31.25 & 30.19 & 32.63 & 16.79 & 28.24 & 15.38 & 39.42 & 18.01 \\
\hline & 3 & 0.00 & 1.67 & 5.97 & 1.35 & 8.43 & 3.85 & 0.00 & 0.00 & 7.55 & 3.16 & 0.00 & 0.00 & 0.69 & 0.00 & 17.74 \\
\hline & 4 & 000 & 1.67 & 0.00 & 1.35 & 1.20 & 0.00 & 0.00 & 0.00 & 0.00 & 1.05 & 2.29 & 6.87 & 2.79 & 3.85 & 7.79 \\
\hline & 5 & 4.54 & 11.67 & 22.39 & 27.02 & 24.09 & 28.20 & 33.75 & 35.00 & 26.41 & 26.31 & 18.32 & 16.79 & 17.48 & 5.77 & 2.15 \\
\hline \multirow[t]{5}{*}{ Zuari } & 1 & 24.24 & 19.04 & 18.51 & 20.83 & 18.36 & 18.86 & 21.15 & 23.21 & 23.88 & 22.41 & 22.75 & 27.12 & 22.16 & 30.19 & 22.42 \\
\hline & 2 & 4.54 & 11.11 & 5.56 & 9.72 & 8.16 & 7.54 & 9.61 & 16.07 & 23.88 & 22.41 & 12.16 & 8.47 & 15.13 & 16.34 & 3.33 \\
\hline & 3 & 6.06 & 4.76 & 12.96 & 16.67 & 0.00 & 3.77 & 5.77 & 5.36 & 5.97 & 10.34 & 22.22 & 5.08 & 21.62 & 20.79 & 9.69 \\
\hline & 4 & 3.03 & 7.94 & 1.85 & 0.00 & 2.04 & 5.66 & 5.77 & 1.78 & 2.98 & 0.00 & 4.76 & 3.39 & 7.02 & 4.95 & 2.42 \\
\hline & 5 & 62.12 & 57.14 & 61.11 & 52.78 & 71.43 & 61.15 & 57.69 & 53.57 & 43.28 & 44.83 & 38.09 & 55.93 & 34.05 & 27.72 & 32.12 \\
\hline \multirow[t]{5}{*}{ I-BASF } & 1 & 40.00 & 34.25 & 36.76 & 38.89 & 40.63 & 32.88 & 34.38 & 30.93 & 38.46 & 36.36 & 68.22 & 66.67 & 59.15 & 63.74 & 64.42 \\
\hline & 2 & 30.00 & 41.10 & 47.06 & 31.94 & 42.19 & 39.73 & 42.71 & 48.45 & 39.23 & 30.30 & 9.35 & 2.47 & 8.45 & 9.89 & 22.09 \\
\hline & 3 & 3.33 & 0.00 & 0.00 & 2.78 & 0.00 & 10.96 & 0.00 & 2.06 & 5.38 & 6.06 & 1.87 & 0.00 & 0.00 & 0.00 & 3.07 \\
\hline & 4 & 0.00 & 0.00 & 0.00 & 4.17 & 0.00 & 0.00 & 2.08 & 3.09 & 2.31 & 2.53 & 0.93 & 0.00 & 2.82 & 0.00 & 0.61 \\
\hline & 5 & 26.67 & 24.66 & 16.18 & 22.22 & 17.19 & 16.44 & 20.83 & 15.46 & 14.62 & 24.75 & 19.63 & 30.86 & 29.58 & 26.37 & 9.82 \\
\hline \multirow{5}{*}{$\begin{array}{l}\text { I-Bayer } \\
\text { Cropscience }\end{array}$} & 1 & 43.75 & 35.85 & 39.78 & 46.23 & 38.67 & 32.91 & 32.98 & 34.07 & 34.00 & 42.35 & 58.82 & 51.56 & 69.44 & 71.93 & 70.47 \\
\hline & 2 & 16.67 & 9.43 & 20.43 & 20.75 & 29.33 & 22.78 & 32.98 & 26.37 & 30.00 & 29.41 & 14.12 & 15.63 & 4.17 & 5.26 & 20.81 \\
\hline & 3 & 10.42 & 30.19 & 19.35 & 9.43 & 8.00 & 10.13 & 4.26 & 4.40 & 14.00 & 9.41 & 0.00 & 0.00 & 4.17 & 3.51 & 4.03 \\
\hline & 4 & 16.67 & 9.43 & 8.60 & 4.72 & 1.33 & 0.00 & 1.06 & 5.49 & 4.00 & 0.00 & 3.53 & 1.56 & 1.39 & 1.75 & 2.01 \\
\hline & 5 & 12.50 & 15.09 & 11.83 & 18.87 & 22.67 & 34.18 & 28.72 & 29.67 & 18.00 & 18.82 & 23.53 & 31.25 & 20.83 & 17.54 & 2.68 \\
\hline \multirow[t]{3}{*}{ I-Monsanto } & 1 & 66.67 & 55.22 & 50.68 & 46.03 & 52.83 & 52.27 & 41.54 & 48.04 & 51.16 & 47.37 & 61.84 & 54.64 & 0.00 & 67.57 & 64.54 \\
\hline & 2 & 20.37 & 16.42 & 30.14 & 17.46 & 16.98 & 15.91 & 32.31 & 34.64 & 32.56 & 32.89 & 14.47 & 13.40 & 0.00 & 16.22 & 17.02 \\
\hline & 3 & 1.85 & 5.97 & 4.11 & 9.52 & 1.89 & 0.00 & 4.62 & 3.35 & 4.65 & 13.16 & 0.00 & 5.15 & 0.00 & 2.70 & 7.09 \\
\hline
\end{tabular}




\begin{tabular}{|c|c|c|c|c|c|c|c|c|c|c|c|c|c|c|c|c|}
\hline & Type & 2002 & 2003 & 2004 & 2005 & 2006 & 2007 & 2008 & 2009 & 2010 & 2011 & 2012 & 2013 & 2014 & 2015 & 2016 \\
\hline & 4 & 3.70 & 2.99 & 1.37 & 11.11 & 1.89 & 0.00 & 4.62 & 6.70 & 3.10 & 6.58 & 2.63 & 2.06 & 0.00 & 3.60 & 6.38 \\
\hline & 5 & 7.41 & 19.40 & 13.70 & 15.87 & 26.42 & 31.82 & 16.92 & 7.26 & 8.53 & 0.00 & 21.05 & 24.74 & 0.00 & 9.91 & 4.96 \\
\hline \multirow[t]{5}{*}{ I-Syngenta } & 1 & 60.53 & 65.79 & 52.63 & 54.55 & $\mathrm{n} / \mathrm{a}$ & $\mathrm{n} / \mathrm{a}$ & 64.71 & 62.22 & 40.48 & 52.00 & $\mathrm{n} / \mathrm{a}$ & 58.75 & 68.09 & 74.60 & 65.00 \\
\hline & 2 & 5.26 & 5.26 & 15.79 & 9.09 & $\mathrm{n} / \mathrm{a}$ & $\mathrm{n} / \mathrm{a}$ & 2.94 & 13.33 & 19.05 & 20.00 & $n / a$ & 11.25 & 4.26 & 4.76 & 25.00 \\
\hline & 3 & 0.00 & 0.00 & 0.00 & 0.00 & $\mathrm{n} / \mathrm{a}$ & $n / a$ & 0.00 & 0.00 & 9.52 & 6.00 & $n / a$ & 1.25 & 2.13 & 1.59 & 1.67 \\
\hline & 4 & 5.26 & 0.00 & 2.63 & 3.03 & $\mathrm{n} / \mathrm{a}$ & $\mathrm{n} / \mathrm{a}$ & 0.00 & 0.00 & 4.76 & 0.00 & $n / a$ & 6.25 & 8.51 & 6.35 & 1.67 \\
\hline & 5 & 28.95 & 28.95 & 28.95 & 33.33 & $\mathrm{n} / \mathrm{a}$ & $\mathrm{n} / \mathrm{a}$ & 32.35 & 24.44 & 26.19 & 22.00 & $n / a$ & 22.50 & 17.02 & 12.70 & 6.67 \\
\hline \multirow[t]{5}{*}{ P-BASF } & 1 & 34.30 & 36.45 & 35.71 & 30.79 & 43.63 & 46.65 & 43.10 & 45.22 & 44.75 & 42.06 & 41.78 & 41.65 & 40.52 & 31.36 & 32.36 \\
\hline & 2 & 46.52 & 40.13 & 37.64 & 39.95 & 29.16 & 29.56 & 37.30 & 34.78 & 34.42 & 35.33 & 30.17 & 32.32 & 31.38 & 41.69 & 37.24 \\
\hline & 3 & 11.71 & 13.04 & 13.19 & 12.47 & 15.55 & 13.16 & 12.23 & 11.83 & 10.33 & 11.40 & 7.35 & 3.05 & 6.03 & 7.63 & 8.78 \\
\hline & 4 & 1.70 & 4.01 & 6.32 & 6.62 & 3.24 & 2.54 & 2.98 & 2.96 & 3.80 & 3.55 & 6.38 & 8.98 & 7.93 & 6.95 & 8.94 \\
\hline & 5 & 5.77 & 6.35 & 7.14 & 10.18 & 8.42 & 8.08 & 4.39 & 5.22 & 6.70 & 7.66 & 14.31 & 14.00 & 14.14 & 12.37 & 12.68 \\
\hline \multirow{5}{*}{$\begin{array}{l}\text { P-Bayer } \\
\text { Cropscience }\end{array}$} & 1 & 34.88 & 45.00 & 37.69 & 43.34 & 37.28 & 39.44 & 43.59 & 43.08 & 38.78 & 30.32 & 42.75 & 41.54 & 36.06 & 35.40 & 34.22 \\
\hline & 2 & 53.49 & 47.50 & 25.24 & 32.97 & 36.87 & 39.91 & 35.09 & 34.88 & 34.78 & 44.93 & 36.27 & 32.88 & 37.65 & 39.31 & 40.07 \\
\hline & 3 & 2.33 & 0.00 & 13.15 & 8.21 & 9.25 & 9.80 & 10.80 & 10.08 & 14.00 & 9.36 & 5.69 & 5.14 & 3.70 & 3.76 & 2.50 \\
\hline & 4 & 0.00 & 0.00 & 2.19 & 5.79 & 6.12 & 6.07 & 4.68 & 5.43 & 4.00 & 6.47 & 7.49 & 9.07 & 9.25 & 8.09 & 8.35 \\
\hline & 5 & 9.30 & 7.50 & 21.74 & 9.69 & 10.48 & 4.78 & 5.83 & 6.53 & 8.44 & 8.92 & 7.80 & 11.37 & 13.34 & 13.44 & 14.86 \\
\hline \multirow[t]{5}{*}{ P-Monsanto } & 1 & 41.38 & 32.89 & 37.74 & 29.43 & 36.48 & 39.70 & 56.04 & 36.17 & 32.66 & 30.31 & 36.42 & 42.81 & 32.74 & 36.98 & 36.23 \\
\hline & 2 & 44.83 & 47.37 & 45.28 & 40.07 & 39.31 & 34.67 & 32.97 & 37.50 & 37.69 & 44.98 & 37.96 & 38.49 & 42.26 & 37.60 & 33.38 \\
\hline & 3 & 13.79 & 7.89 & 5.66 & 14.54 & 10.69 & 13.82 & 6.59 & 8.51 & 9.05 & 13.51 & 5.97 & 11.51 & 11.29 & 9.86 & 12.27 \\
\hline & 4 & 0.00 & 6.58 & 6.60 & 4.96 & 3.14 & 2.51 & 3.30 & 2.66 & 3.77 & 3.79 & 8.29 & 6.47 & 6.77 & 5.55 & 8.56 \\
\hline & 5 & 0.00 & 5.26 & 4.72 & 10.99 & 10.38 & 9.30 & 1.10 & 15.16 & 16.83 & 7.41 & 11.37 & 0.72 & 6.94 & 10.02 & 9.56 \\
\hline \multirow[t]{5}{*}{ P-Syngenta } & 1 & 51.15 & 41.50 & 32.73 & 38.79 & 23.26 & 26.84 & 35.27 & 41.39 & 35.74 & 37.21 & 40.75 & 35.21 & 34.02 & 35.18 & 31.81 \\
\hline & 2 & 37.31 & 43.54 & 55.76 & 45.79 & 47.67 & 49.85 & 31.78 & 21.98 & 28.16 & 30.62 & 21.13 & 27.46 & 35.19 & 31.41 & 32.82 \\
\hline & 3 & 6.54 & 4.76 & 4.85 & 8.41 & 15.89 & 7.37 & 2.71 & 1.47 & 3.97 & 2.71 & 1.89 & 3.17 & 3.52 & 6.03 & 4.58 \\
\hline & 4 & 5.00 & 4.08 & 3.03 & 2.34 & 5.04 & 5.01 & 7.36 & 2.56 & 2.17 & 3.49 & 9.06 & 4.58 & 6.16 & 7.04 & 9.92 \\
\hline & 5 & 0.00 & 6.12 & 3.64 & 4.67 & 8.14 & 10.91 & 22.87 & 32.60 & 29.96 & 25.97 & 27.17 & 29.58 & 21.11 & 20.35 & 20.87 \\
\hline
\end{tabular}




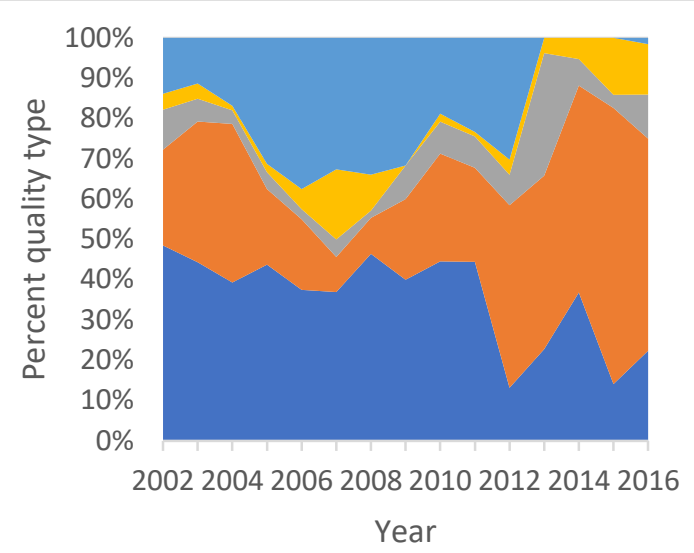

ロType 1 Type 2 Type 3 Type 4 Type 5

Figure 9. Quality composition of disclosures from Coromandel from 2002-2016. Type $1(p=0.003128)$, Type $2(p=0.011438)$, Type $3(p=0.229464)$, Type 4 $(p=0.236308)$, Type $5(p=0.067912)$

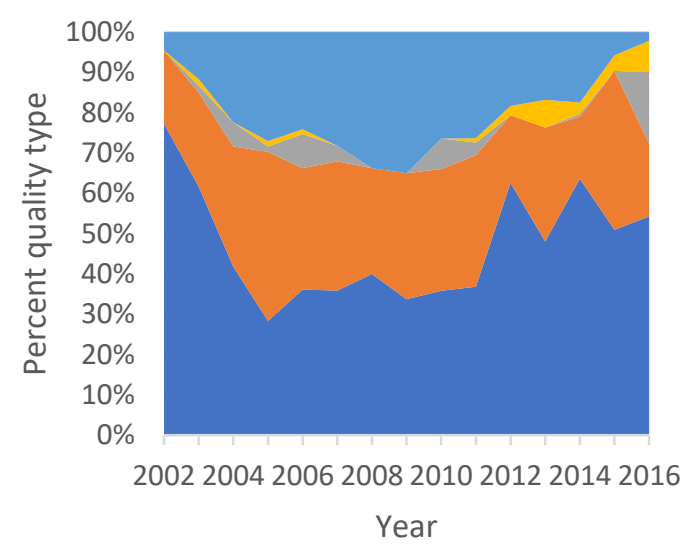

-Type 1 -Type 2 - Type 3 Type 4 -Type 5

Figure 11. Quality composition of disclosure from UPL from 2002-2016. Type 1 ( $p=0.872292)$, Type 2 $(p=0.635764)$, Type $3(p=0.462509)$, Type 4 $(p=0.003868)$, Type $5(p=0.416638)$

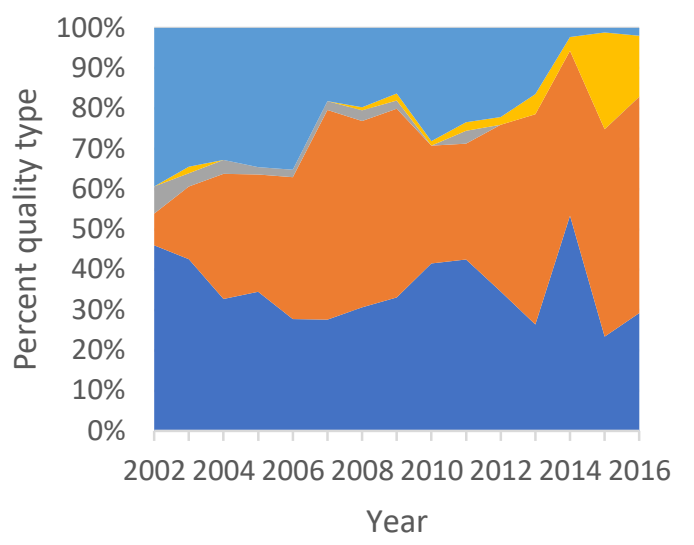

-Type 1 -Type 2 -Type 3 - Type 4 - Type 5

Figure 10. Quality composition of disclosures from Rallis India Ltd from 2002-2016. Type 1 $(p=0.495358)$, Type $2(p=0.00222)$, Type 3 $(p=0.00024)$, Type $4(p=0.005455)$, Type 5 $(p=8.65 E-06)$

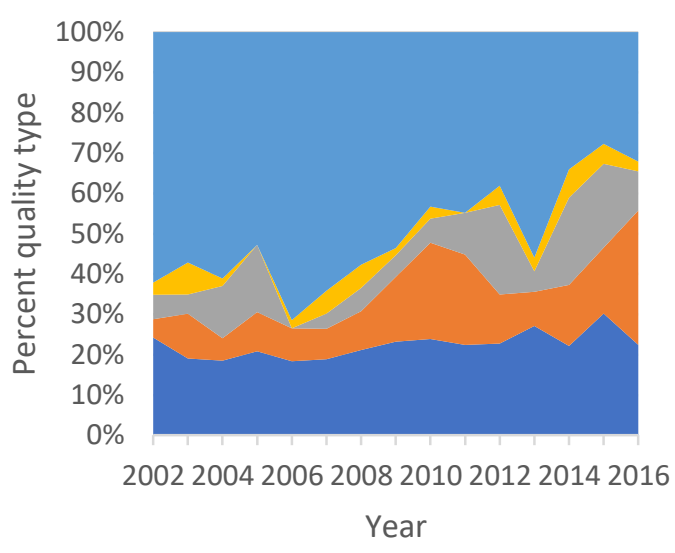

-Type 1 Type 2 Type 3 - Type 4 - Type 5

Figure 12. Quality composition of disclosure from Zuari from 2002-2016. Type 1 ( $p=0.02035)$, Type 2 $(p=0.005926)$, Type $3(p=0.097645)$, Type 4 $(p=0.787839)$, Type $5(p=0.000238)$ 


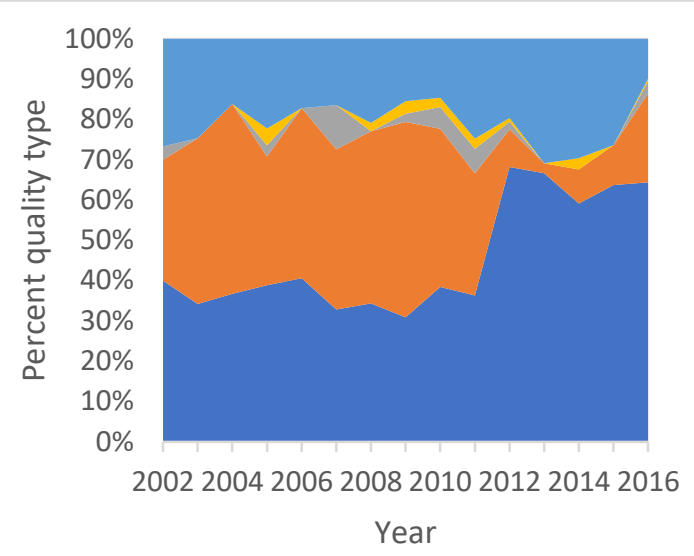

ロType 1 Type 2 Type 3 Type 4 Type 5

Figure 13. Quality composition of disclosure from IBASF from 2002-2016. Type $1(p=0.001141)$, Type 2 $(p=0.004024)$, Type $3(p=0.83187)$, Type $4(p=$ $0.701068)$, Type $5(p=0.913945)$

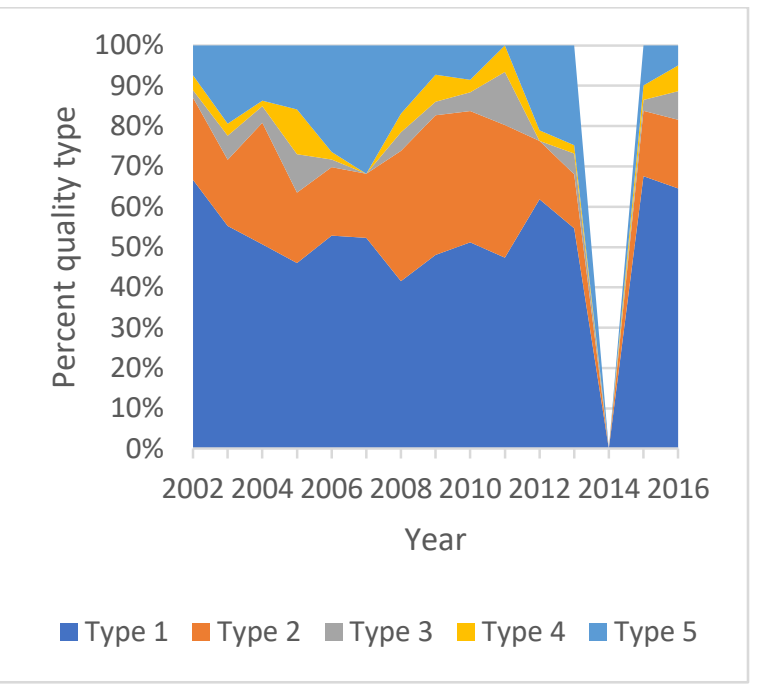

Figure 15. Quality composition of disclosure from IMonsanto from 2002-2016. Type $1(p=0.637622)$, Type 2 ( $p=0.329595)$, Type $3(p=0.996855)$, Type 4 $(p=0.897474)$, Type $5(p=0.236979)$

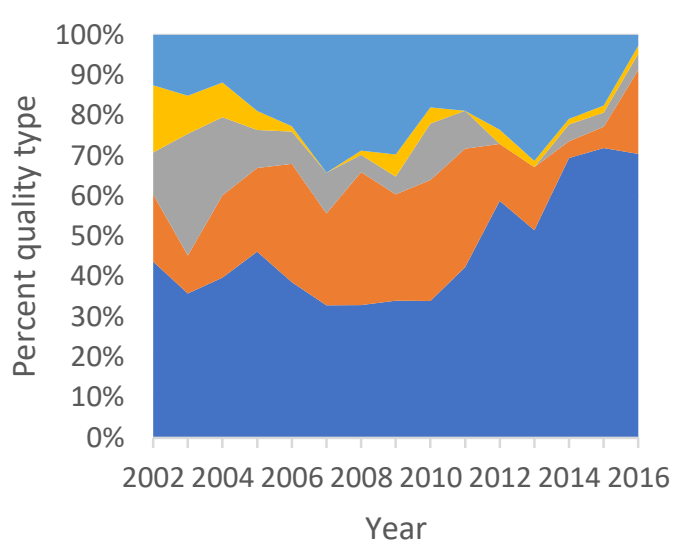

-Type 1 -Type 2 -Type 3 - Type 4 - Type 5

Figure 14. Quality composition of disclosure from I-Bayer from 2002-2016. Type $1(p=0.001916)$, Type $2(p=0.396966)$, Type $(p=0.005518)$, Type 4 $(p=0.006641)$, Type $5(p=0.990807)$

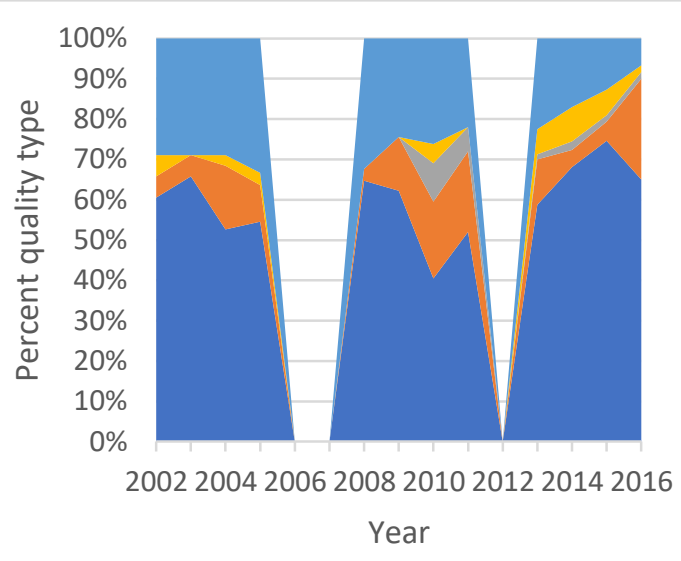

- Type 1 Type $2 \square$ Type 3 - Type 4 - Type 5

Figure 16. Quality composition of disclosure from I-Syngenta from 2002-2016. Type $1(p=0.573692)$, Type $2(p=0.305055)$, Type $3(p=0.215392)$, Type 4 $(p=0.239045)$, Type $5(p=0.11977)$ 


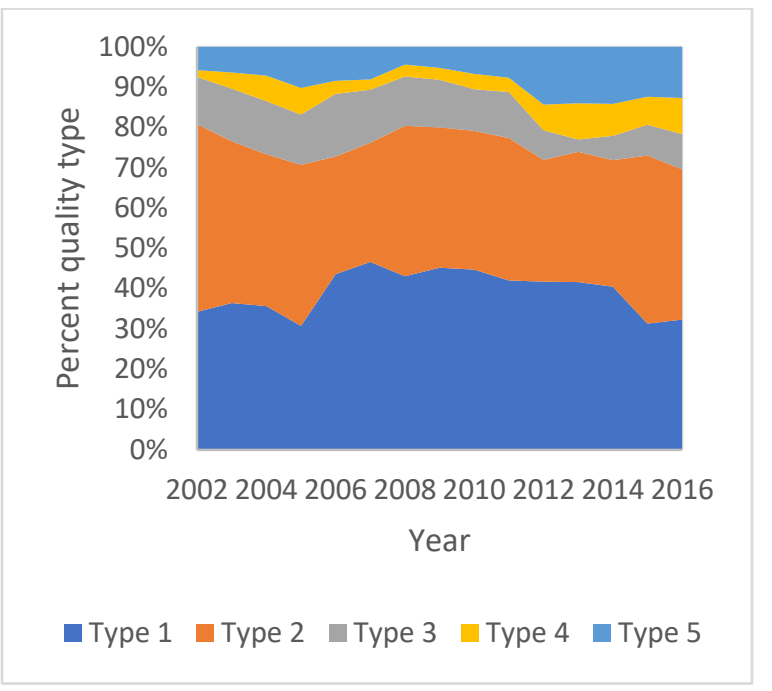

Figure 17. Quality composition of disclosure from $P$-BASF from 2002-2016. Type $1(p=0.914266)$, Type $2(p=0.213162)$, Type $3(p=0.00116)$, Type 4 $(p=0.009394)$, Type $5(p=0.00334)$

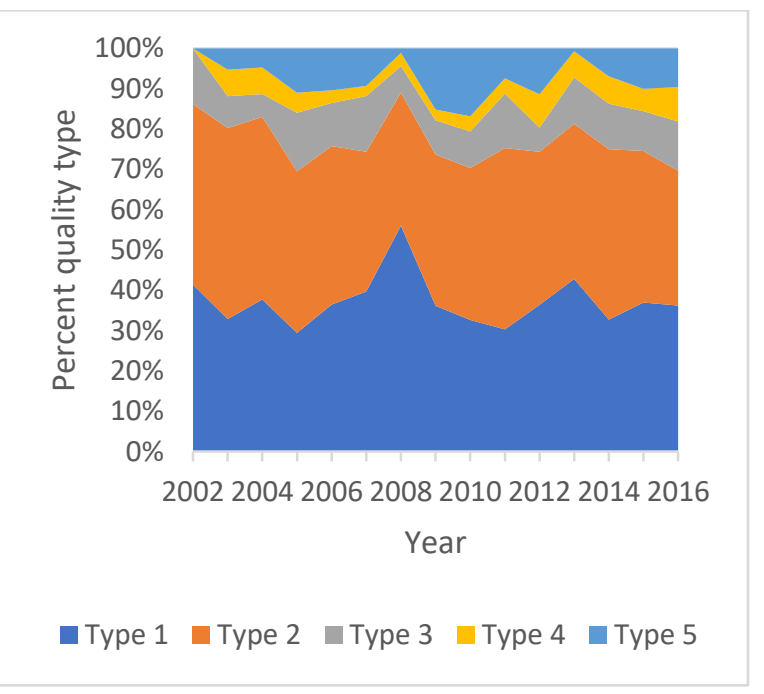

Figure 19. Quality composition of disclosure from $P$-Monsanto from 2002-2016. Type $1(p=0.82403)$, Type $2(p=0.0595)$, Type $3(p=0.926843)$, Type 4 $(p=0.042729)$, Type $5(p=0.354159)$

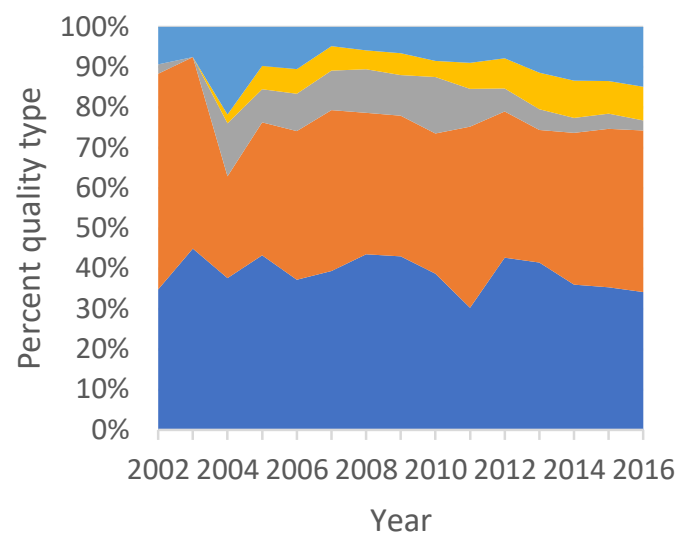

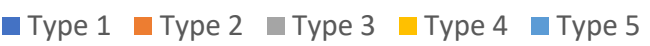

Figure 18. . Quality composition of disclosure from $P$-Bayer from 2002-2016. Type 1 ( $p=0.25242)$, Type $2(p=0.534258)$, Type $3(p=0.55123)$, Type 4 $(p=3.16 E-05)$, Type 5 ( $p=0.576412)$

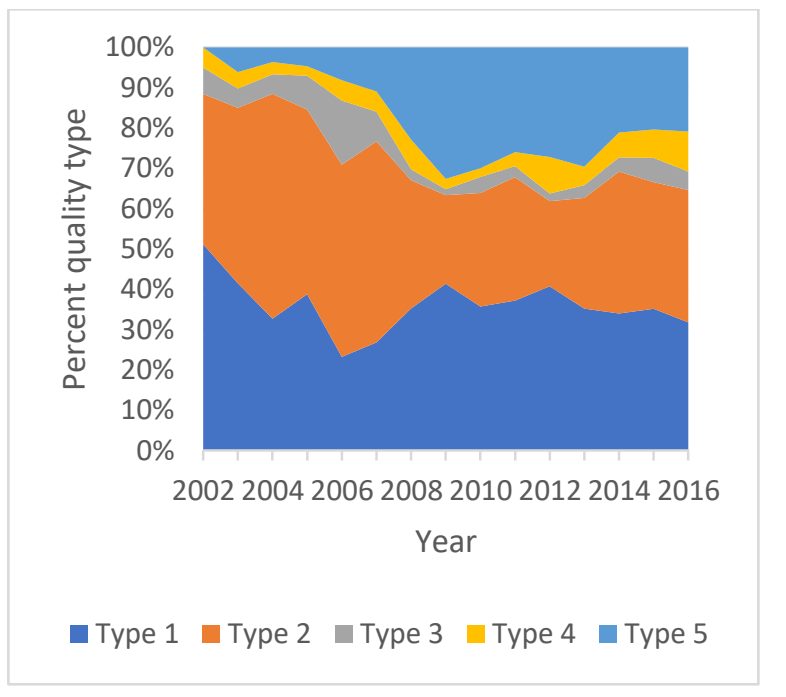

Figure 20. Quality composition of disclosure from $P$-Syngenta from 2002-2016. Type 1( $p=0.345442)$, Type $2(p=0.013754)$, Type $3(p=0.168384)$, Type 4 $(p=0.038067)$, Type $5(p=0.001349)$

If the corporation category is a significant factor, this indicates that there is a lack of standardization among the reporting practices of the three corporation categories. In the absence of standardization, 
the raw data was consulted to identify where the lack of standardization was most prevalent among the three categories.

\subsubsection{Diversity}

Results for the analysis of the interaction between year and corporation category on CED diversity showed that there was not a statistically significant interaction $(p=0.5781)$. Results from the main effects show that there is statistical significance for the effects of corporation category $(p=<0.0001)$ and year $(p=0.0007)$. This indicates that the year and corporation category both significantly affect the diversity of CEDs, while the lack of interaction indicates that the difference between year and corporation category has not significantly changed.

Therefore, it can be concluded that the category of a corporation is a significant factor influencing the diversity of that corporations' CEDs and this has not lessened over time. Knowing that the diversity of CEDs from the three corporation categories is not standardized and observing the data in figure 4, it can be seen that the diversity of domestic corporations and I-MNCs are similar to one another, while P-MNC is much different. Therefore, in the absence of standardization, I-MNC disclose more similarly to local corporations rather than the parent corporation.

\subsubsection{Quantity}

Results for the analysis of the interaction between year and corporation category on CED quantity showed that there was not a statistically significant interaction $(p=0.6183)$. Results from the main effects show that there is statistical significance for the effects of corporation category $(p=<0.0001)$ and year $(p=<0.0001)$ on CED quantity.

From the above, it can be determined that the quantity of disclosures in a CED report is significantly affected by the year of publication and the category of the corporation. Additionally, because the interaction between the year and corporation category is not significant, it can be interpreted that the 
corporation category has been a significant factor over the entirety of the timeframe. Again, in the absence of standardization, I-MNC disclose more similarly to local corporations rather than the parent corporations.

\subsubsection{Quality}

\subsection{Type 1}

Results for the analysis of the interaction between the year and corporation category on CED quality Type 1 showed that there was not a statistically significant interaction between the year and corporation category $(p=0.6961)$. Results from the main effects show that there is statistical significance for the effects of corporation category $(p=<0.0001)$ and year $(p=<0.0001)$ on CED quality Type 1 . In the case of Type 1 disclosures, raw data suggest that, in accordance with a lack of standardization, domestic corporations (2002: 44.2\%, 2016: 36.6\%) are more similar to P-MNC (2002:39.3\%, 2016: 33.9\%) than to I-MNC (2002: 52.0\%, 2016: 66.3\%).

\subsection{Type 2}

Results for the analysis of the interaction between the year and corporation category on CED quality Type 2 showed that there was not a statistically significant interaction between the year and corporation category $(p=0.2201)$. Results from the main effects show that there is statistical significance for the effects of corporation category $(p=<0.0001)$ and year $(p=0.0010)$ on CED quality Type 2 . In 2002, the percentage of Type 2 disclosures from domestic corporations (2002: 13.7\%, 2016: 35.7\% ) seemed to more closely relate to I-MNCs $(2002: 19.5 \%, 2016: 20.7 \%$ ), but by 2016 was more similar to P-MNCs (2002: 44.2\% 2016: 36.0\%).

\subsection{Type 3}

Results for the analysis of the interaction between the year and corporation category on CED quality Type 3 showed that there was not a statistically significant interaction between the year and 
corporation category $(p=0.9230)$. Results from the main effects show that there is statistical significance for the effects of corporation category $(p=<0.0001)$ but not the year $(p=0.1647)$ on CED quality Type 3 . Type 3 quality disclosure appeared to be more similar between domestic corporations (2002: 7.2\%, 2016: 11.0\%) and P-MNCs (2002: 9.9\%, 2016: 7.5\%) than to I-MNC (2002: 4.0\%, 2016: 4.3\%).

\subsection{Type 4}

Results for the analysis of the interaction between the year and corporation category on CED quality Type 4 showed that there was a statistically significant interaction between the year and corporation category $(p=0.0015)$. Results from the main effects show that there is statistical significance for the effects of corporation category $(p=<0.0001)$ and year $(p=<0.0001)$ on CED quality Type 4 . For Type 4 quality disclosures, domestic corporations (2002: $2.2 \%, 2016$ : 9.4\%) follow a more similar trend to PMNC (2002: 2.5\%, 2016: 8.8\%) than to I-MNCs (2002: 6.0\%, 2016: $2.7 \%)$.

\subsection{Type 5}

Results for the analysis of the interaction between the year and corporation category on CED quality Type 5 showed that there was a statistically significant interaction between the year and corporation category $(p=0.0015)$. Results from the main effects show that there is statistical significance for the effects of corporation category $(p=<0.0001)$ and year $(p=0.0023)$ on CED quality Type 5 . For Type 5 quality disclosures, domestic corporations (2002: 32.7\%, 2016: 7.3\%) disclose in a way that is more similar to I-MNCS (2002: 18.5\%, 2016: 6.0\%) than P-MNCs (2002: 4.1\%, 2016: 13.7\%).

\subsection{Overall}

In the results for quality Types, 1 and 2 both the main effects are statistically significant, while the interaction between effects is not. For quality Type 3, neither the interaction nor the year is significant factors. However, the effect of the corporation category remains statistically significant. Quality Type 4 and 5 show a different trend; for these factors both the main effects and the interaction is significant, 
suggesting that while the year and corporation category have a significant effect on the portion of highquality disclosures this difference may be changing over time. While results show that there is a statistically significant difference between the quality proportions of disclosures from the three types of corporations, analysis of the data trends are inconclusive as to whether domestic corporations are reporting in accordance with local norms and practices or in line with their parent corporation.

\subsection{Discussion}

This study aimed to identify significant changes in the CEDs from three categories of corporations over the span of fifteen years. The purpose was to identify if corporations were continuing to improve the diversity, quantity, and quality of their CEDs over time. Since CED is a relatively new area of reporting, only gaining momentum in the last 40 years (Lin, 2008), examining a timeframe of fifteen years can provide deep insight into the continued development of reporting practices.

The results partially support hypothesis $1 \mathrm{a}$ and fully support hypothesis $1 \mathrm{~b}$, which stated:

H1a: Each corporation categories studied will increase the diversity of environmental disclosures over the timeframe examined.

H1b: Each corporation categories studied will increase the quantity of environmental disclosures over the timeframe examined.

The reason hypothesis 1a was only partially supported is that while all corporations showed a trend of increasing diversity, only two of the corporation categories; P-MNCs and domestic corporations, showed a statistically significant increase in diversity. I-MNCs also showed an increase in diversity of CEDs, but this was not statistically supported overall, despite being statistically significant for two of the four corporations within the category. All corporation categories showed a statistically significant increase in the quantity of CEDs for the fifteen-year timeframe and all were found to be statistically significant. 
Legitimacy theory provides insight as to why the diversity and quantity of disclosures increased over time. Legitimacy theory, as discussed in section 2.5 , states that corporations disclose to meet the expectations of society in order to maintain or obtain corporate legitimacy (Ashford \& Gibbs, 1990; Dowling \& Pfeffer, 1975; Suddaby et al., 2017). Public awareness of corporate environmental impacts has increased over the years, in turn increasing the expectations of society for enhanced corporate responsibility (Alrazi et al., 2015). When society's expectations are higher, corporations will have to increase disclosure in order to maintain legitimacy. Increased disclosure suggests that corporations are perceiving higher expectations from the society in which they operate and responding to the diversity and quantity of their disclosures.

If we accept this explanation, the next point of interest would be the increased rate at which P-MNCs responded in comparison to the other two corporate categories. In terms of diversity, all categories began the timeframe with reasonably similar scores in 2002 (domestic: 10, I-MNC: 9.25 \& P-MNC: 11). However, P-MNCs increased the diversity of their CEDs at a rate that was higher than I-MNCs and domestic corporations. The increase in CEDs by P-MNCs is consistent with the expectations of the literature, which has shown growing levels of reporting amongst large corporations (Ali et al., 2017; Lu \& Abeysekera, 2014; Ortas et al., 2014). One possible explanation for this may be society's increasing expectations for reporting. Given the relatively high public visibility of many P-MNCs, they may be more susceptible to public pressure to enhance reporting (Ali et al., 2017; Ortas et al., 2014.

Another possible explanation could be that, although all corporations were exposed to increased societal expectations for CEDs, only the P-MNCs had the resources to respond (or, at least, they had more resources to respond than did the other two categories of corporations)(Qiu et al., 2016; Lu \& Abeysekera, 2014; Ortas et al., 2014). I-MNCs and domestic corporations failed to report on diversity categories related to the interests of local stakeholders, such as pressure groups (PRESS), information retrieval process (IRP), and pollution (POLL). This indicates that there may be a lack of expectation for 
these types of disclosures in the society that I-MNCs and domestic corporations are operating in. This is not completely unexpected, as the education and general awareness level of environmental impacts of the public in Indian societies are not as high as North American or European societies (Ali et al., 2017). Ali et al. (2017) state that corporations in developing countries are less influenced by local stakeholders and more by an external stakeholder, who would have less interest in disclosure focused on local concerns.

Quantity revealed the same trend, i.e. that P-MNCs increased their quantity at a higher rate then I-MNCs and domestic corporations. However, in this scenario, P-MNCs also had higher CED quantity at the beginning of the timeframe. Quantity or volume is a common measurement used in CED research and past studies have shown the quantity of CEDs to increase over time (Gibson \& O'Donovan, 2007) and that corporations in developing countries tend to have fewer CEDs (Momin, 2006)

Hypothesis 1c was not supported by the results of this study, which stated the following:

H1c: Each corporation categories studied will increase the quality of environmental disclosures over the timeframe examined.

Results from the regression analysis were inconclusive regarding the quality of CEDs over time. Individually, corporations showed a significant increase or decrease in certain quality types. However, these individual trends were not consistent across all corporations in a category. This would be an interesting aspect for future research, perhaps on a larger sample size.

A secondary aim of this study was to assess the standardization of reporting practices across the corporate categories. As previously discussed, standardization of environmental disclosures within a multinational corporation leads to many benefits, through improved corporate legitimacy, including higher profits, better stock prices, lower pressure from non-government organizations and reduced liability of foreignness (Arora \& Lodhia, 2017; Cho \& Patten, 2007; Hunter \& Bansal, 2006). In addition to 
benefits within the corporation itself, increased standardization among corporations can lead to better information for stakeholders and investors. Standardization will also likely be a goal when designing future disclosure requirements (KPMG, 2017). Therefore, understanding how corporations are reporting now within the different categories will help to inform the future decision regarding regulation.

Results from this analysis revealed support for hypothesis $2 a$ and $2 b$, which stated:

H2a: The diversity of environmental disclosures of P-MNCs and I-MNCs will lack standardization, and the environmental disclosures of I-MNCs will be more similar to those of domestic corporations than the P-MNC.

H2b: The quantity of environmental disclosures of P-MNCs and I-MNCs will lack standardization, and the environmental disclosures of I-MNCs will be more similar to those of domestic corporations than the PMNC.

Results from the two-way repeated measures ANOVA indicated that corporate category was a significant factor affecting the diversity and quantity of the CEDs. Further examination of the data reveals that domestic corporations and I-MNCs have the most similar CED reporting practices, while the reporting practices of P-MNCs differ. This suggests that I-MNCs follow localized reporting norms, which was also the key conclusion in Wilson (2013).

Although the quality was found to be significantly affected by the corporation category by the ANOVA analysis, it could not be determined conclusively that the I-MNCs were reporting more similarly to domestic corporations. This is because the disclosure quality of domestic corporations appeared to relate more similarly to P-MNCs. Therefore, the results did not support $\mathrm{H} 2 \mathrm{c}$, which states: 
H2c: The quality of environmental disclosures of P-MNCs and I-MNCs will lack standardization, and the environmental disclosures of I-MNCs will be more similar to those of domestic corporations than the PMNC.

The lack of standardization within the reporting practices of different categories of corporations also fits within the framework of legitimacy theory. Previous research has found conflicting results on the influence of foreign ownership on CEDs. Results from this study, however, suggest that having foreign ownership does not significantly influence CEDs. Based on the framework provided by legitimacy theory, two categories of corporations will disclose similarly if they are experiencing similar societal expectation. The corporations do this to appease relevant stakeholders and ensure their own corporate success by acquiring legitimacy. If two corporate categories are not exposed to the same societal expectations, they will not disclose in a standardized way because they do not need to in order to acquire the same legitimacy. Subsidiaries are a special case because these corporations are influenced by the same society as domestic corporations but also have the potential to be influenced by their parent corporations and the stakeholders that become involved when a corporation has foreign ownership, such as investors, international consumers, and potentially certification bodies.

Results found that I-MNCs reported more similarly to domestic corporations showing the disclosure practices are localized not standardized. This indicates being a subsidiary of a multinational corporation did not significantly influence the societal expectations for these corporations and that I-MNCs and domestic corporations are exposed to similar societal expectations from the public, government, customers, and other domestic stakeholders. Had the I-MNCs been found to disclose in a way that was more similar to P-MNCs this would have suggested that expectations for these corporations were higher than those for domestic corporations.. This means that these two categories of corporations are exposed to similar societal expectations from the public, government, customers, and other domestic stakeholders. However, in the future, as globalization continues to increase, it may be seen that both 
domestic and I-MNC increase the diversity and quantity of CEDs to be more comparable to those of PMNCs.

This study has looked at the theoretical framework of legitimacy theory, specifically legitimacy-asproperty as defined by Suchman (1995). In this perspective, organizations acquire legitimacy from society (Suddaby et al., 2017). The generalized response from corporations in this study over time was to increase the diversity and quantity of their CEDs in an attempt to maintain their possession of legitimacy. The lack of standardization or generalization, as defined by Suchman (1995), between corporations in the three different categories indicates that corporations are likely operating in systems of different norms. To this point Suddaby et al. (2017, pg. 457) state, "Complying with social pressures of legitimacy enhances the survivability of an organization and, as a result, creates a high degree of similarity among organizations in a common organizational field". This helps explain why greater standardization was observed between domestic corporations, as they were operating in the same organizational environment.

\subsection{Conclusion}

This study found that, over a period of fifteen years, all corporations had significant changes in the quantity of CEDs. P-MNC and domestic corporations had significant changes in diversity. As well, the portion of the quality type in CEDs was inconclusive, though there were instances of statically significance. Results also showed that the corporation category was a significant factor affecting the diversity, quantity, and quality, indicating that there is a lack of standardization among the reporting practices of the different categories of corporations. The results further suggest that in terms of diversity and quantity, subsidiary corporations conform more to localized reporting practices. The results were inconclusive for the analysis of quality. 
This research is significant because it addressed environmental disclosures of foreign subsidiaries, with both domestic corporations and parent corporations for context, providing an efficient means to measure standardization. Standardization of environmental disclosure in multinational corporations has been shown to improve corporate policy, auditing, performance targets, better satisfy stakeholders and reduce the liability of foreignness (Hunter \& Bansal, 2007; Christmann, 2004). Improved CEDs can provide better insight into the operations, environmental initiatives, and auditing of agrochemical corporations. As previously established, the agrochemical industry has the potential to be particularly dangerous to both the environment and human health (Aktar et al., 2009). As well, comprehensive disclosures have the potential to improve policy and auditing within the corporation, although there is no confirmed correlation with environmental performance (Meng et al., 2014; Hunter \& Bansal, 2007; Christmann, 2004; Hughes et al., 2001). This research provides insight into whether or not multinational corporations are implementing comprehensive disclosure practices throughout the entirety of their operations. The results can also be used to inform decisions to implement programs to improve environmental disclosure in multinational corporations or in developing countries. Additionally, this study is believed to be one of the first to analyze corporate environmental disclosure of single multinational corporations operating in different countries.

This study contributes to both academic research and policy, but limitations must also be considered. In this research, a limited sample size was used due to the use of manual coding, which is highly intensive. Furthermore, the content analysis itself has limitations, such as being limited to available materials and sample size due to the time-consuming nature, and debates are ongoing over the reliability of content analysis methods. Efforts to increase the reliable use of content analysis in this study included coder practice and inter and intra- coder reliability testing.

Regardless, this study does offer valuable insights into the CED practices of multinational, subsidiary, and domestic corporations and provides a foundation for future research. Such research could include 
exploring the motivations for aligning multinational and subsidiary CEDs. Although motivations for environmental reporting have been widely studied, there is less research specifically focused on understanding how stakeholder influences differ based on the location of operation and how this impacts the results of environmental disclosure by multinationals operating in multiple countries. Additionally, researchers have highlighted the need for further research on the quality of CEDs. Although the results from this study were inconclusive regarding the quality of the CEDs, future research featuring a larger sample size may produce significant results. 


\section{Appendices}

\section{Appendix A: Extended Methodology}

\section{A.1 Corporation Selection}

Table A. 1: Profiles of Corporations Selected for Research

\begin{tabular}{|c|c|c|c|c|}
\hline Category & Corporation & Location & $\begin{array}{l}\text { Revenue for } \\
\text { FY16* }\end{array}$ & $\begin{array}{l}\text { Number of } \\
\text { Employees for } \\
\text { FY16 }\end{array}$ \\
\hline \multirow[t]{4}{*}{$\begin{array}{l}\text { Parent } \\
\text { Multinational }\end{array}$} & $\begin{array}{l}\text { Monsanto } \\
\text { Corporation }\end{array}$ & USA & 13,502 & 20,800 \\
\hline & BASF SE & Germany & 57,550 & 113,830 \\
\hline & $\begin{array}{l}\text { Bayer Cropscience } \\
\text { AG }\end{array}$ & Germany & 46,769 & 115,200 \\
\hline & Syngenta AG & Switzerland & 12,790 & 27,810 \\
\hline \multirow[t]{4}{*}{$\begin{array}{l}\text { Subsidiary } \\
\text { Multinational }\end{array}$} & $\begin{array}{l}\text { Monsanto India } \\
\text { Ltd }\end{array}$ & India & 123.7 & 268 \\
\hline & BASF India Ltd & India & $10,232.94$ & 1,868 \\
\hline & $\begin{array}{l}\text { Bayer Cropscience } \\
\text { Ltd (India) }\end{array}$ & India & 5460.00 & 1,126 \\
\hline & Syngenta India Ltd & India & 440.56 & Not disclosed \\
\hline \multirow[t]{4}{*}{ Domestic } & Rallis & India & 330.18 & 952 \\
\hline & Coromandel & India & $2,164.81$ & 4,309 \\
\hline & Zuari & India & 728.24 & 727 \\
\hline & $\begin{array}{l}\text { United } \\
\text { Phosphorus }\end{array}$ & India & 3141.11 & 3,489 \\
\hline
\end{tabular}

* In USD in millions

\section{A.2 Document Selection}


Corporations can publish environmental information in many ways and through several forms of media, including annual reports, standalone reports, web pages, media, etc. For the purpose of this research, information that is published by the company in either annual reports or standalone sustainability reports will be analyzed. For the companies selected, many publish only annual reports which typically have environmental information integrated; others publish an annual report and a sustainability report [Table A.2]

Only publicly available information was collected, typically through the company's webpage. In some instances, links to sustainability reports were faulty or missing, in these cases a request was sent to the company via email. If companies were able to provide the report, it was included, otherwise reports not readily accessible to the public were not included. An overview of sustainability reports included in the analysis is featured in Table A.3. Additionally, standalone reports not pertaining to environment or sustainability, for example, compensation reports, were not included.

Table A. 2: Length of annual reports published by selected corporations in number of pages

\begin{tabular}{|l|l|l|l|l|l|}
\hline & 2012 & 2013 & 2014 & 2015 & 2016 \\
\hline P-BASF & 244 & 252 & 276 & 262 & 260 \\
\hline P-Syngenta & 70 & 72 & 68 & 72 & 74 \\
\hline $\begin{array}{l}\text { P-Monsanto } \\
\text { Science }\end{array}$ & 110 & 109 & 114 & 109 & 118 \\
\hline $\begin{array}{l}\text { I-BASF } \\
\text { P-BayerCrop }\end{array}$ & 285 & 351 & 340 & 361 & 344 \\
\hline $\begin{array}{l}\text { I-Syngenta } \\
\text { N/A }\end{array}$ & 88 & 88 & 120 & 92 & 120 \\
\hline
\end{tabular}




\begin{tabular}{|l|l|l|l|l|l|}
\hline & 2012 & 2013 & 2014 & 2015 & 2016 \\
\hline I-Monsanto & 96 & 100 & 126 & 132 & 148 \\
\hline $\begin{array}{l}\text { I-BayerCrop } \\
\text { Science }\end{array}$ & 70 & 78 & 96 & 108 & 135 \\
\hline $\begin{array}{l}\text { United } \\
\text { Phosphorus }\end{array}$ & 188 & 184 & 107 & 228 & 167 \\
\hline \begin{tabular}{l} 
Rallis Chemical \\
\hline
\end{tabular} & 128 & 144 & 168 & 181 & 265 \\
\hline Coromandel & 116 & 140 & 164 & 172 & 244 \\
\hline Zuari & 128 & 126 & 159 & 176 & 218 \\
\hline
\end{tabular}

Table A. 3: Overview of Standalone sustainability reports published by corporations indicated by page length

\begin{tabular}{|l|l|l|l|l|l|}
\hline & 2012 & 2013 & 2014 & 2015 & 2016 \\
\hline P-BASF & N/A & N/A & N/A & N/A & N/A \\
\hline P-Syngenta & N/A & N/A & N/A & N/A & N/A \\
\hline $\begin{array}{l}\text { P-Monsanto } \\
\text { P-BayerCrop }\end{array}$ & 153 & $26+19^{*}$ & 168 & 117 & 129 \\
\hline I-BASF & N/A & N/A & N/A & N/A & N/A \\
\hline
\end{tabular}




\begin{tabular}{|l|l|l|l|l|l|}
\hline & 2012 & 2013 & 2014 & 2015 & 2016 \\
\hline I-Syngenta & N/A & N/A & N/A & N/A & N/A \\
\hline I-Monsanto & N/A & N/A & N/A & N/A & N/A \\
\hline $\begin{array}{l}\text { I-BayerCrop } \\
\text { Science }\end{array}$ & N/A & N/A & N/A & N/A & N/A \\
\hline $\begin{array}{l}\text { United } \\
\text { Phosphorus }\end{array}$ & N/A & Not available & Not available \\
\end{tabular}

*In 2013 P-Monsanto published spring and fall sustainability reports

N/A indicates a lack of publication

† United Phosphorus' website indicates there are sustainability reports for these years, however the links are faulty, United Phosphorus did not respond to requests for these reports.

\section{A.3 CONI coding steps}

CONI is unique because it allows the user to rate a unit, in this case, a sentence, on three criteria at once. CONI measures the diversity, quality and quantity of a document by recording the category of disclosure, the quality of the information, and the overall amount of relevant information. The unit for measurement in this method is a sentence, therefore any sentence containing relevant corporate environmental disclosure (CED) will be analyzed according to the following steps.

\section{A.3.1 Diversity Coding}


The first step in the process is to determine the diversity category best suited to the relevant sentence.

Beck et al. (2010) initially developed 12 Categories and 48 sub-categories for diversity coding. This study, however, will use the adapted categories developed by Wilson (2013), which features 18 categories and 59 sub-categories [Table A.4]. All sentences containing CED information are classified into a category and sub-category based on the overall meaning of the sentence. If the coder is unable to determine a singular meaning for the sentence then the sentence will be classified based into the category relevant to the first point made in the sentence.

Table A. 4: Category and sub-category of diversity coding with examples

\begin{tabular}{|c|c|c|}
\hline Category & Sub-Category & Example \\
\hline \multirow[t]{4}{*}{$\begin{array}{l}\text { GEN: General environmental } \\
\text { information }\end{array}$} & $\begin{array}{l}\text { 1: Any general mention, } \\
\text { including the use of keywords, } \\
\text { i.e., environment, nature, } \\
\text { climate, etc. }\end{array}$ & $\begin{array}{l}\text { "As the world's leading } \\
\text { chemical company, we combine } \\
\text { economic success with } \\
\text { environmental protection and } \\
\text { social responsibility." (BASF } \\
\text { Annual report, 2016, pg. 19) }\end{array}$ \\
\hline & $\begin{array}{l}\text { 2: Aims, mentioning } \\
\text { environmental goals, aims or } \\
\text { commitments }\end{array}$ & $\begin{array}{l}\text { "We set ourselves ambitious } \\
\text { goals with voluntary } \\
\text { commitments and monitor our } \\
\text { performance in terms of the } \\
\text { environment, health and safety } \\
\text { using our Responsible Care } \\
\text { Management System." (BASF } \\
\text { Annual report, 2016, pg. 24) }\end{array}$ \\
\hline & $\begin{array}{l}\text { 3: Management Systems \& } \\
\text { Processes }\end{array}$ & $\begin{array}{l}\text { "The Company continues its } \\
\text { environment and safety } \\
\text { initiatives and has successfully } \\
\text { implemented internationally } \\
\text { recognized } \\
\text { Environment \& Safety } \\
\text { Standards and is an ISO 14001: } \\
2004 \text { and OHSAS 18001:2007 } \\
\text { certified organization." (Zuari } \\
\text { Annual Report, 2016, pg. 5) }\end{array}$ \\
\hline & $\begin{array}{l}\text { 4: Disclosure guidelines, } \\
\text { including GRI }\end{array}$ & $\begin{array}{l}\text { "The Bayer Group's } \\
\text { sustainability reporting is } \\
\text { aligned to the g3.1 guidelines of }\end{array}$ \\
\hline
\end{tabular}




\begin{tabular}{|c|c|c|}
\hline Category & Sub-Category & Example \\
\hline & & $\begin{array}{l}\text { the Global Reporting Initiative } \\
\text { (GRI) and the ten principles of } \\
\text { the UN Global Compact } \\
\text { (UNGC)." (Bayer Annual report, } \\
\text { 2014, pg. 31) }\end{array}$ \\
\hline & $\begin{array}{l}\text { 5: Environmental Initiatives, i.e., } \\
\text { REACH, International Chemical } \\
\text { Environmental Initiatives } \\
\text { (excluding Responsible care) }\end{array}$ & $\begin{array}{l}\text { "REACH is a European Union } \\
\text { regulatory framework for the } \\
\text { registration, evaluation and } \\
\text { authorization of chemicals, and } \\
\text { will be implemented gradually } \\
\text { until 2018." (BASF Annual } \\
\text { report, 2014, pg. 236) }\end{array}$ \\
\hline & $\begin{array}{l}\text { 6: Results from environmental } \\
\text { audits, policies, etc., including } \\
\text { environmental incidents }\end{array}$ & $\begin{array}{l}\text { "However, looking at the } \\
\text { resource intensity - total use } \\
\text { per dollar operating income } \\
\text { (\$EBIT) - we have again } \\
\text { observed an overall } \\
\text { improvement in our } \\
\text { environmental performance." } \\
\text { (Syngenta Annual Review 2012, } \\
\text { pg. 41) }\end{array}$ \\
\hline & $\begin{array}{l}\text { 7: Long-term policy, goals } \\
\text { and/or strategy }\end{array}$ & $\begin{array}{l}\text { "Most importantly, we } \\
\text { approach sustainability as a } \\
\text { long-term, collaborative effort." } \\
\text { (Monsanto CSR Report, } 2016 \\
\text { pg. 4) }\end{array}$ \\
\hline & 8: Continuous Improvement & $\begin{array}{l}\text { "We continue to enhance our } \\
\text { environmental reporting by } \\
\text { improving data quality and } \\
\text { adding sites to the reporting } \\
\text { scope." (Syngenta Annual } \\
\text { Review, 2013, pg. 41) }\end{array}$ \\
\hline $\begin{array}{l}\text { RES: } \\
\text { Those responsible for the } \\
\text { environmental behaviour or } \\
\text { implementation of activities }\end{array}$ & $\begin{array}{l}\text { 1: Top management } \\
\text { A. Committee/ department } \\
\text { B. Individual/ Membership } \\
\text { C. Aims and objectives }\end{array}$ & $\begin{array}{l}\text { A: "It starts at the top with the } \\
\text { Sustainability and Corporate } \\
\text { Responsibility Committee of our } \\
\text { board of directors, which } \\
\text { reviews and monitors our } \\
\text { sustainability performance." } \\
\text { (Monsanto CSR Report, 2014, } \\
\text { pg. 13) }\end{array}$ \\
\hline
\end{tabular}




\begin{tabular}{|c|c|c|}
\hline Category & Sub-Category & Example \\
\hline & & $\begin{array}{l}\text { B: “A 24-year agriculture sector } \\
\text { veteran, Dr. Shukla has served } \\
\text { successfully in varied roles of } \\
\text { increasing responsibility } \\
\text { spanning Technology } \\
\text { Development, Regulatory, Sales } \\
\text { \& Customer Service, } \\
\text { Sustainability and Corporate } \\
\text { Affairs across the India and Asia } \\
\text { Pacific regions; he was also the } \\
\text { Country Manager of the } \\
\text { Monsanto Company's business } \\
\text { in Indonesia where he re- } \\
\text { established the Roundup }{ }^{\circledR} \\
\text { business and helped lay the } \\
\text { foundation for the maize } \\
\text { business." (Monsanto India } \\
\text { Annual Review, 2013, pg. 24) } \\
\text { C: N/A }\end{array}$ \\
\hline & 2: Results & $\begin{array}{l}\text { "Our newly formed Office of } \\
\text { Sustainability is a cross- } \\
\text { functional team of employees } \\
\text { who have day-to-day } \\
\text { responsibilities in core areas of } \\
\text { sustainability including } \\
\text { stakeholder engagement, food } \\
\text { and nutrition security, } \\
\text { environmental management, } \\
\text { safety, health, supply chain, } \\
\text { global policy, business conduct } \\
\text { and human rights." (Monsanto } \\
\text { CSR Report, 2014, pg. 13) }\end{array}$ \\
\hline & $\begin{array}{l}\text { 3: Anybody working with the } \\
\text { organization, i.e., reference to } \\
\text { employees }\end{array}$ & $\begin{array}{l}\text { "For Occupational and process } \\
\text { safety as well as health and } \\
\text { environmental protection and } \\
\text { corporate security, we rely on } \\
\text { comprehensive preventative } \\
\text { measures as well as on the } \\
\text { involvement of all employees } \\
\text { and contractors" (BASF Annual }\end{array}$ \\
\hline
\end{tabular}




\begin{tabular}{|c|c|c|}
\hline Category & Sub-Category & Example \\
\hline & & Report, 2016, pg. 98) \\
\hline \multirow[t]{3}{*}{ POLL } & $\begin{array}{l}\text { 1: Air } \\
\text { A. Emissions (Actual and Result) } \\
\text { B. Action/ Target }\end{array}$ & $\begin{array}{l}\text { A: “[Chart format] Total } \mathrm{CO}_{2} \\
\text { Emissions (000s tonnes); 2014: } \\
\text { 1,730, 2013: 1,710, 2012: } \\
\text { 1,574.” (Syngenta Annual } \\
\text { review, 2014, pg. 56) } \\
\text { B: “In line with our Group target } \\
\text { we are endeavouring to reduce } \\
\text { specific greenhouse gas } \\
\text { emissions (total emissions } \\
\text { divided by the manufactured } \\
\text { sales volume) by 20\% through } \\
\text { 2020." (Bayer Annual Report, } \\
\text { 2015, pg. 136) }\end{array}$ \\
\hline & $\begin{array}{l}\text { 2: Water } \\
\text { A. Emissions (Actual and Result) } \\
\text { B. Action/ Target }\end{array}$ & $\begin{array}{l}\text { A: "A total of 1,644 million cubic } \\
\text { meters of water were } \\
\text { discharged from BASF } \\
\text { production sites in 2016, } \\
\text { including } 184 \text { million cubic } \\
\text { meters of wastewater from } \\
\text { production." (BASF Annual } \\
\text { report, 2016, pg. 108) } \\
\text { B: "In order to avoid } \\
\text { unanticipated emissions and the } \\
\text { pollution of surface or } \\
\text { groundwater, we create water } \\
\text { protection strategies for our } \\
\text { production sites." (BASF Annual } \\
\text { report, 2016, pg. 108) }\end{array}$ \\
\hline & $\begin{array}{l}\text { 3: Waste } \\
\text { A. Emitted (Actual and Result) } \\
\text { B. Action/ Target } \\
\text { C. Recycling (must include the } \\
\text { word recycle) }\end{array}$ & $\begin{array}{l}\text { A: "Total waste volume } \\
\text { amounted to } 2.10 \text { million metric } \\
\text { tons in } 2016 \text { (+3.7\%)." (BASF } \\
\text { Annual report, 2016, pg. 110) } \\
\text { B: "Waste prevention is our } \\
\text { topmost goal." (BASF Annual } \\
\text { report, 2016, pg. 110) } \\
\text { C: "If waste is unavoidable, we } \\
\text { review the options for recycling } \\
\text { or energy recovery, using }\end{array}$ \\
\hline
\end{tabular}




\begin{tabular}{|c|c|c|}
\hline Category & Sub-Category & Example \\
\hline & & $\begin{array}{l}\text { BASF's existing Verbund } \\
\text { structures for efficient waste } \\
\text { management." (BASF Annual } \\
\text { report, 2016, pg. 110) }\end{array}$ \\
\hline & $\begin{array}{l}\text { 4: Land } \\
\text { A. Emission (Actual \& Result) } \\
\text { B. Action/ Target }\end{array}$ & $\begin{array}{l}\text { A: “[Chart format] Total waste; } \\
\text { Landfill; FY14 8,600; FY15 } \\
\text { 7,060” (Monsanto CSR Report, } \\
\text { 2015, pg. 76) } \\
\text { B: N/A }\end{array}$ \\
\hline & $\begin{array}{l}\text { 5: Emission and Pollution } \\
\text { related to products }\end{array}$ & $\begin{array}{l}\text { "In 2016-17, CO2 emissions per } \\
\text { tonne of production in UPL } \\
\text { manufacturing plants reduced } \\
\text { by } 22 \% \text { compared to } 2015-16 . " \\
\text { (UPL Annual Report, 2016) }\end{array}$ \\
\hline \multirow[t]{2}{*}{$\begin{array}{l}\text { PROD: } \\
\text { Product disclosures }\end{array}$} & $\begin{array}{l}\text { 1: Product Stewardship, Life } \\
\text { cycle analysis, Packaging }\end{array}$ & $\begin{array}{l}\text { "Our seed and crop protection } \\
\text { products are subject to our Life } \\
\text { Cycle Stewardship Activities } \\
\text { Management Process } \\
\text { (LCStAMP)." (Monsanto CSR } \\
\text { Report, 2014, pg. 34) }\end{array}$ \\
\hline & $\begin{array}{l}\text { 2: Mention of environmentally } \\
\text { friendly products, product } \\
\text { production, or application }\end{array}$ & $\begin{array}{l}\text { "The process takes place at } \\
\text { room temperature and under } \\
\text { normal atmospheric conditions, } \\
\text { resulting in energy savings and } \\
\text { greater environmental } \\
\text { compatibility." (BASF annual } \\
\text { report, 2016, pg. 8) }\end{array}$ \\
\hline \multirow[t]{2}{*}{$\begin{array}{l}\text { SUST: } \\
\text { Sustainability disclosure }\end{array}$} & $\begin{array}{l}\text { 1: General mention of } \\
\text { sustainability }\end{array}$ & $\begin{array}{l}\text { "As a responsible business } \\
\text { corporation, we have built } \\
\text { sustainable and effective CSR } \\
\text { initiatives that are vital towards } \\
\text { fulfilling critical societal needs in } \\
\text { the communities we operate } \\
\text { in." (Zuari Annual report, 2016, } \\
\text { pg. 43) }\end{array}$ \\
\hline & $\begin{array}{l}\text { 2: Commitment to UNCED, } \\
\text { Kyoto, External Environmental } \\
\text { Rating, etc. }\end{array}$ & $\begin{array}{l}\text { "In November 2015, Monsanto } \\
\text { signed the White House } \\
\text { American Business Act on } \\
\text { Climate Pledge, which affirmed }\end{array}$ \\
\hline
\end{tabular}




\begin{tabular}{|c|c|c|}
\hline Category & Sub-Category & Example \\
\hline & & $\begin{array}{l}\text { our commitment to climate } \\
\text { action and our support for a } \\
\text { strong outcome from the } \\
\text { United Nations Framework } \\
\text { Convention on Climate Change } \\
\text { Conference in Paris (COP21) } \\
\text { that took place in December } \\
\text { 2015." (Monsanto CSR Report, } \\
\text { 2015, pg 53) }\end{array}$ \\
\hline & $\begin{array}{l}\text { 3: Biodiversity conservation, } \\
\text { habitat and species }\end{array}$ & $\begin{array}{l}\text { "We develop remediation } \\
\text { solutions that combine nature } \\
\text { conservation, climate } \\
\text { protection concerns, costs, and } \\
\text { social responsibility." (BASF } \\
\text { Annual report, 2016, pg. 110) }\end{array}$ \\
\hline & 4: Mention of climate change & $\begin{array}{l}\text { "As a company entirely devoted } \\
\text { to agriculture, we are } \\
\text { particularly attuned to the } \\
\text { potential effects of climate } \\
\text { change including drought, } \\
\text { severe weather, rising sea } \\
\text { levels, shifting pest infestations, } \\
\text { compromised harvests and } \\
\text { flooding." (Monsanto CSR } \\
\text { Report, 2015, pg. 52) }\end{array}$ \\
\hline \multirow[t]{3}{*}{$\begin{array}{l}\text { LIAB: } \\
\text { Environmental Liability }\end{array}$} & 1: Financial liability & $\begin{array}{l}\text { "During the year provision has } \\
\text { been utilised to the extent of } \\
\text { Rs. } 32 \text { Lacs. It is expected that a } \\
\text { significant portion of this } \\
\text { provision would be utilised in } \\
\text { next financial year and all will } \\
\text { have been utilised within five } \\
\text { years after the reporting date." } \\
\text { (Syngenta Annual Report, 2013, } \\
\text { pg. 58) }\end{array}$ \\
\hline & 2: Disclosure on Balance sheet & $\begin{array}{l}\text { "[Chart format] Power, Fuel and } \\
\text { water; 2017: 20.352.31 2016: } \\
\text { 26045.86" (Zuari, 2016, pg. 90) }\end{array}$ \\
\hline & 3: Justification for no disclosure & $\mathrm{n} / \mathrm{a}$ \\
\hline
\end{tabular}




\begin{tabular}{|c|c|c|}
\hline Category & Sub-Category & Example \\
\hline \multirow[t]{5}{*}{$\begin{array}{l}\text { ACT: } \\
\text { Environmental Activities }\end{array}$} & 1: Staff Training & $\begin{array}{l}\text { "The Company has on-going } \\
\text { training programs on } \\
\text { Environment covering all levels } \\
\text { of employees" (UPL Limited } \\
\text { Annual report, 2014, pg. 61) }\end{array}$ \\
\hline & $\begin{array}{l}\text { 2: Project Involvement (without } \\
\text { partnership) }\end{array}$ & $\begin{array}{l}\text { "The Prince Edward Island } \\
\text { Stream Restoration Project in } \\
\text { Canada supports stream } \\
\text { environments and reduces the } \\
\text { risk of soil runoff by planting } \\
\text { strips of vegetation along the } \\
\text { edges of farm properties and } \\
\text { waterways." (Syngenta Annual } \\
\text { review, 2014, pg. 13) }\end{array}$ \\
\hline & 3: Awards & $\begin{array}{l}\text { "In addition, BASF was one of } \\
24 \text { companies in } 2016 \text {, out of a } \\
\text { total of } 607 \text { assessed by CDP, to } \\
\text { receive the top grade of "A" for } \\
\text { sustainable water management, } \\
\text { putting it among the world's } \\
\text { leading enterprises in this area." } \\
\text { (BASF Annual report, 2016, pg. } \\
\text { 14) }\end{array}$ \\
\hline & 4: Sponsoring & $\begin{array}{l}\text { "Our people were proud to be a } \\
\text { co-sponsor with the Howard G. } \\
\text { Buffett Foundation of two } \\
\text { important farmer workshops on } \\
\text { cover crop education." } \\
\text { (Monsanto CSR Report, 2012, } \\
\text { pg. 103) }\end{array}$ \\
\hline & $\begin{array}{l}\text { 5: Partnerships with } \\
\text { environmental organizations } \\
\text { (must use word partner/ } \\
\text { partnership) }\end{array}$ & $\begin{array}{l}\text { "The Europe-wide standard } \\
\text { came into force at the end of } \\
2011 \text { and was developed by } \\
\text { nongovernmental } \\
\text { organizations, governments and } \\
\text { businesses under the direction } \\
\text { of the independent organization } \\
\text { European Water Partnership } \\
\text { (EWP)." (BASF Annual report, } \\
\text { 2016, pg. 237) }\end{array}$ \\
\hline
\end{tabular}




\begin{tabular}{|c|c|c|}
\hline Category & Sub-Category & Example \\
\hline \multirow[t]{3}{*}{$\begin{array}{l}\text { BRR: } \\
\text { Business Risk }\end{array}$} & $\begin{array}{l}\text { 1: Environmental risks related } \\
\text { to business }\end{array}$ & $\begin{array}{l}\text { "Major chemicals such as } \\
\text { glyphosate (herbicide), atrazine } \\
\text { (herbicide), chlorpyrifos } \\
\text { (insecticide) and others are } \\
\text { constantly under review, facing } \\
\text { the risk of being phased out or } \\
\text { banned if more environment- } \\
\text { friendly alternatives become } \\
\text { available." (UPL Limited Annual } \\
\text { report, 2014, pg. } 37 \text { ) }\end{array}$ \\
\hline & $\begin{array}{l}\text { 2: Attempts to manage or } \\
\text { reduce environmental risks }\end{array}$ & $\begin{array}{l}\text { "We aim to reduce potential } \\
\text { risks in the areas of } \\
\text { environment, safety and } \\
\text { security, health protection, } \\
\text { product stewardship, } \\
\text { compliance, and labor and } \\
\text { social standards by setting } \\
\text { ourselves globally uniform } \\
\text { requirements that frequently go } \\
\text { beyond legal requirements." } \\
\text { (BASF Annual report, 2016, pg. } \\
\text { 29) }\end{array}$ \\
\hline & 3: Related costs & $\begin{array}{l}\text { "Additionally, during the } \\
\text { current year, the Company has } \\
\text { recognised the business } \\
\text { interruption claim amounting to } \\
\text { '2500 lakhs received from the } \\
\text { Insurance company on account } \\
\text { of the 'Hudhud' cyclone in } \\
\text { Vishakapatnam unit." } \\
\text { (Coromandel Annual Report, } \\
2015, \text { pg. 123) }\end{array}$ \\
\hline \multirow[t]{2}{*}{$\begin{array}{l}\text { PRESS: } \\
\text { Pressure Groups }\end{array}$} & 1: Shareholders & $\begin{array}{l}\text { "In } 2013 \text { we continued our } \\
\text { dialogue with current and } \\
\text { potential investors who base } \\
\text { their investment decisions on } \\
\text { ESG criteria." (Bayer Annual } \\
\text { report, 2013, pg. 41) }\end{array}$ \\
\hline & 2: Stakeholders & $\begin{array}{l}\text { "The trend toward more } \\
\text { sustainability in our customer } \\
\text { industries continues. We want }\end{array}$ \\
\hline
\end{tabular}




\begin{tabular}{|c|c|c|}
\hline Category & Sub-Category & Example \\
\hline & & $\begin{array}{l}\text { to use innovations to take } \\
\text { advantage of the resulting } \\
\text { opportunities." (BASF Annual } \\
\text { Review, 2016, pg. 117) }\end{array}$ \\
\hline & 3: Government & $\begin{array}{l}\text { "With the Government's focus } \\
\text { shifting towards improving } \\
\text { water and nutrients use } \\
\text { efficiency by making higher } \\
\text { budgetary allocation towards } \\
\text { micro irrigation, Business } \\
\text { foresees significant growth } \\
\text { opportunities, going forward." } \\
\text { (Coromandel Annual Report, } \\
\text { 2016, pg. 35) }\end{array}$ \\
\hline \multirow[t]{2}{*}{$\begin{array}{l}\text { SER: } \\
\text { Separate Environmental Report }\end{array}$} & $\begin{array}{l}\text { 1: Separate report references in } \\
\text { the annual report }\end{array}$ & $\begin{array}{l}\text { "In addition to our integrated } \\
\text { corporate report, we publish } \\
\text { further information about } \\
\text { sustainability issues online. } \\
\text { Links to this supplementary } \\
\text { information are provided in } \\
\text { each chapter." (BASF Annual } \\
\text { Review, 2012, pg. 4) }\end{array}$ \\
\hline & 2: Contact details & N/A \\
\hline $\begin{array}{l}\text { RC: } \\
\text { Responsible Care }\end{array}$ & $\begin{array}{l}\text { Any mention of Responsible } \\
\text { Care }\end{array}$ & $\begin{array}{l}\text { "We act responsibly as an } \\
\text { integral part of society and have } \\
\text { set out the framework for our } \\
\text { voluntary commitments in our } \\
\text { Responsible Care Management } \\
\text { System." (BASF Annual report, } \\
\text { 2016. Pg. 96) }\end{array}$ \\
\hline \multirow[t]{2}{*}{$\begin{array}{l}\text { ENE: } \\
\text { Energy Disclosure }\end{array}$} & $\begin{array}{l}\text { 1: Conservation/ energy saving } \\
\text { attempts (Goals \& Results) }\end{array}$ & $\begin{array}{l}\text { "The Verbund system is an } \\
\text { important component of our } \\
\text { energy efficiency strategy: } \\
\text { Waste heat from one plant's } \\
\text { production process is used as } \\
\text { energy in other plants." (BASF } \\
\text { Annual report, 2016, pg. 105) }\end{array}$ \\
\hline & $\begin{array}{l}\text { 2: Energy use, development and } \\
\text { exploration of alternative }\end{array}$ & $\begin{array}{l}\text { "We are exploring the use of } \\
\text { renewable energies. These can } \\
\text { only become a permanent part }\end{array}$ \\
\hline
\end{tabular}




\begin{tabular}{|c|c|c|}
\hline Category & Sub-Category & Example \\
\hline & energy & $\begin{array}{l}\text { of our energy mix if they are } \\
\text { competitive in terms of supply } \\
\text { security and cost." (BASF } \\
\text { Annual report, 2016, pg. 105) }\end{array}$ \\
\hline & 3: Energy usage & $\begin{array}{l}\text { "[Chart format] Energy (TJ); } \\
\text { 2014: 9,930, 2013: 10.202, } \\
\text { 2012: 9,336" (Syngenta Annual } \\
\text { review, 2014, pg. 56) }\end{array}$ \\
\hline \multirow[t]{2}{*}{$\begin{array}{l}\text { INP: } \\
\text { Resource Input }\end{array}$} & 1: Water Input & $\begin{array}{l}\text { "Reduced specific water } \\
\text { consumption in FY2016-17 by } \\
6 \% \text { i.e. achieved } 0.59 \mathrm{~kL} \\
\text { water/kL of product from } 0.63 \\
\mathrm{~kL} \text { water/kL of product." } \\
\text { (Monsanto India Annual Report, } \\
2016, \text { pg. 105) }\end{array}$ \\
\hline & $\begin{array}{l}\text { 2: Resource Input, renewable, } \\
\text { non-renewable, toxic, etc. }\end{array}$ & $\begin{array}{l}\text { "Sourcing of surfactant for } \\
\text { Roundup } 41 \% \text { SL in local tankers } \\
\text { in place of HDPE drums. This } \\
\text { has helped us reduce HDPE } \\
\text { consumption by over } 60 \text { MT per } \\
\text { annum." (Monsanto India } \\
\text { Annual Report, 2016, pg. 105) }\end{array}$ \\
\hline $\begin{array}{l}\text { SUPP: } \\
\text { Supplier Related Disclosure }\end{array}$ & $\begin{array}{l}\text { Mention of sustainable supply } \\
\text { chain, environmental } \\
\text { requirements and/or supplier } \\
\text { compliance }\end{array}$ & $\begin{array}{l}\text { "Both new and existing } \\
\text { suppliers are selected and } \\
\text { evaluated not only on the basis } \\
\text { of economic criteria, but also on } \\
\text { environmental, social and } \\
\text { corporate governance } \\
\text { standards." (BASF Annual } \\
\text { report, 2016, pg. 92) }\end{array}$ \\
\hline \multirow[t]{2}{*}{$\begin{array}{l}\text { COMP: } \\
\text { Compliance Disclosure }\end{array}$} & $\begin{array}{l}\text { 1: Compliance with } \\
\text { environmental laws, regulations } \\
\text { and/or voluntary compliance } \\
\text { with initiatives }\end{array}$ & $\begin{array}{l}\text { "Due diligence includes, for } \\
\text { example, reviewing risk- } \\
\text { relevant factors such as } \\
\text { compliance with applicable } \\
\text { environmental regulations and } \\
\text { occupational health and safety } \\
\text { standards at production sites." } \\
\text { (Bayer Annual report, 2014, pg. } \\
\text { 218) }\end{array}$ \\
\hline & 2: Non-compliance, including & "In the United States, Bayer is \\
\hline
\end{tabular}




\begin{tabular}{|c|c|c|}
\hline Category & Sub-Category & Example \\
\hline & fines and lawsuits & $\begin{array}{l}\text { one of numerous parties } \\
\text { involved in a series of claims } \\
\text { brought by federal and state } \\
\text { environmental protection } \\
\text { agencies." (Bayer Annual report, } \\
2014 \text {, pg. 325) }\end{array}$ \\
\hline $\begin{array}{l}\text { IRP: } \\
\text { Information Retrieval Process }\end{array}$ & $\begin{array}{l}\text { The process to obtain feedback } \\
\text { from stakeholders }\end{array}$ & $\begin{array}{l}\text { "The Trust of customers and } \\
\text { consumers is essential for the } \\
\text { successful introduction of new } \\
\text { technologies. That is why we } \\
\text { enter into dialog with } \\
\text { stakeholders at an early stage of } \\
\text { development." (BASF Annual } \\
\text { Review, 2016, pg. 118) }\end{array}$ \\
\hline OTHER & $\begin{array}{l}\text { Disclosure not fitting in the } \\
\text { above categories, i.e., } \\
\text { transportation, transparency, } \\
\text { accountability, etc. }\end{array}$ & $\begin{array}{l}\text { "Transport is handled by } \\
\text { logistics service suppliers that } \\
\text { are selected according to } \\
\text { stringent safety, environmental } \\
\text { and quality criteria." (Bayer } \\
\text { Annual Report, 2015, pg. 108) }\end{array}$ \\
\hline $\begin{array}{l}\text { ECE: } \\
\text { External Environmental Factors }\end{array}$ & $\begin{array}{l}\text { Mention of weather-related } \\
\text { information, including droughts } \\
\text { and rainfall but excluding } \\
\text { related business risks }\end{array}$ & $\begin{array}{l}\text { "In 2012, the cause was more } \\
\text { abrupt, with significant } \\
\text { production shortfalls due to } \\
\text { weather." (Syngenta Annual } \\
\text { Review 2012, pg. 6) }\end{array}$ \\
\hline
\end{tabular}

Table A. 5: Classification categories for qualitative coding

\begin{tabular}{|l|l|l|}
\hline Disclosure Type & Example & Explanation \\
\hline Type 1 & $\begin{array}{l}\text { "Most importantly, we approach } \\
\text { sustainability as a long-term, collaborative } \\
\text { effort." (Monsanto CSR Report, 2016 pg. 4) }\end{array}$ & $\begin{array}{l}\text { This sentence is vague and includes } \\
\text { no goal or plan for execution; } \\
\text { because of this it offers little value } \\
\text { and is classified as a Type 1. }\end{array}$ \\
\hline
\end{tabular}




\begin{tabular}{|l|l|l|}
\hline Type 2 & $\begin{array}{l}\text { "In November 2015, Monsanto signed the } \\
\text { White House American Business Act on } \\
\text { Climate Pledge, which affirmed our } \\
\text { commitment to climate action and our } \\
\text { support for a strong outcome from the } \\
\text { United Nations Framework Convention on } \\
\text { Climate Change Conference in Paris } \\
\text { (COP21) that took place in December } \\
\text { 2015." (Monsanto CSR Report, 2015, pg. } \\
\text { 53) }\end{array}$ & $\begin{array}{l}\text { This disclosure offers information } \\
\text { beyond a general level, including a } \\
\text { specific commitment to and named } \\
\text { authority. Note the use of the year } \\
\text { does not count as quantitative } \\
\text { information and therefore does not } \\
\text { make this disclosure appropriate for } \\
\text { Type 4 classification; it is instead } \\
\text { classified as a Type 2. }\end{array}$ \\
\hline Type 3 & $\begin{array}{l}\text { "The Group's total energy consumption } \\
\text { meanwhile was even down 2.8\% at 80.8 } \\
\text { petajoules." (Bayer Annual report, 2013, } \\
\text { pg. 132" }\end{array}$ & $\begin{array}{l}\text { This disclosure offers primarily } \\
\text { quantitative information with little } \\
\text { narration or explanation. }\end{array}$ \\
\hline Type 4 & $\begin{array}{l}\text { "Additionally, during the current year, the } \\
\text { interruption claim amounting to '2500 } \\
\text { lakhs received from the Insurance company } \\
\text { on account of the 'Hudhud' cyclone in } \\
\text { Vishakapatnam unit." (Coromandel Annual } \\
\text { Report, 2015, pg 123) }\end{array}$ & $\begin{array}{l}\text { This sentence includes an element of } \\
\text { both quantitative and qualitative } \\
\text { information, but doesn't compare to } \\
\text { any additional years; for this reason, } \\
\text { it is classified as a Type 4 disclosure. }\end{array}$ \\
\hline $\begin{array}{l}\text { Type 5 } \\
\text { 2015, po0; FY15 7,060" (Monsanto CSR Report, }\end{array}$ & $\begin{array}{l}\text { This chart entry contains data for the } \\
\text { financial year 2014 and 2015; } \\
\text { because a Type 5 disclosure doesn't } \\
\text { require a narrative component, this } \\
\text { classifies as a Type 5. }\end{array}$ \\
\hline
\end{tabular}

\section{A. 3.3 User Reliability}

To ensure the reliability of coding throughout the entirety of the research intra-coder reliability will be used. Furthermore, inter-coder reliability will also be tested for the incorporation of data coded by Wilson between 2002 and 2011. Based on the results from the coder reliability, the data will be merged and treated as a single dataset including results from 2002 to 2016. Reliability testing will be done by 
using a test-retest method where the author will recode ten percent of the data set for the intra-coder reliability test and seven percent of the dataset for the inter-coder reliability test. The difference in retest percent is due to the size of data set samples. Recommended retest sample size varies greatly depending on the number of coders, the number of variables and the reliability test being used, however, 5 to 10 percent is typically accepted as a guideline (De Swert, 2012).

Following the retest, the reliability will be calculated using Krippendorff's alpha. Previously, CONI methods have performed well on coder reliability test despite the complexity of the procedures; Beck et al. reported a Krippendorff's alpha score of 0.97 and Wilson scored 0.88 (Beck et al., 2010 \& Wilson, 2013). Typically, a score above 0.80 using Krippendorff's alpha is considered high and acceptable (De Swert, 2012).

\section{A.4 Analysis}

The analysis will be performed separately on measurements of diversity, quality, and quantity data to preserve the multifaceted advantage of using the CONI method. Following the analysis, the results will be logically analyzed in relation to each aspect to provide a comprehensive analysis of the corporations.

\section{A.4.1 Diversity}

To score a corporation on diversity each report was given a score out of $18 ; 18$ being the total number of categories that were available to be reported on. For categories that a corporation did report on there was a value of one given; if a corporation failed to report within a category a value of zero was given. Dividing the total sum of diversity in the report by 18 provides a number up to one that is referred to as the diversity index (Wilson, 2013). To report on an overall category of corporations or year the diversity index scores for the appropriate dataset can simply be averaged.

These index scores will be used to illustrate the trend over the timeframe for P-MNC, I-MNC and domestic corporations. A one-tailed paired t-test will also be run to test for significance over time, as 
well as a regression analysis. Also, to determine if the diversity of I-MNC disclosures is more similar to PMNC or domestic, a one-tailed two-sample t-test will be run to determine if there are significant differences between the categories in terms of diversity.

\section{A.4.2 Quality}

In order to effectively express the quality profile of a report, the quality types from 1-5 were scored as a percentage based on the total CED quantity. This calculation is based on the following formula adapted from Wilson (2013).

$$
\text { Type } x \text { quality percentage }=\frac{\text { Quantity of type } x \text { CED sentences }}{\text { Total CED Quantity }} * 100
$$

These percentages will be used to show the trend over the timeframe for P-MNC, I-MNC and domestic corporations. A one-tailed paired t-test will also be run to test for significance over time, as well as a regression analysis. Also, to determine if the quality of I-MNC disclosures is more similar to P-MNC or domestic, a one-tailed two-sample t-test will be run to determine if there are significant differences between the categories in terms of quality.

\section{A.4.3 Quantity}

To determine the quantity of CED disclosure in a report the total CED sentence count was summed. The total quantity will be used to illustrate the trend over the timeframe for P-MNC, I-MNC and domestic corporations. A one-tailed paired t-test will also be run to test for significance over time, as well as a regression analysis. Also, to determine if the quantity of I-MNC disclosures is more similar to P-MNC or domestic, a one-tailed two-sample t-test will be run on specific categories to determine if there is 
significant difference between the categories in terms of diversity and whether the I-MNC use a disclosure strategy that is more similar to P-MNC or domestic corporations.

\section{Appendix B: Extended Results \& Discussion}

\section{B.1 Analysis of CED Diversity}

\section{B.1.1 Diversity of disclosures from Domestic Corporations}

In 2002 the average diversity index score for the four domestic corporations was 10 out of a possible 18. By 2016 the average score was 13.5 [Table B.1]. The diversity scores per company increased relatively consistently, however, both Coromandel and Rallis Indian Ltd have high periods of diversity between 2002-2004 and 2007-2009, respectively [Figure B.1]. For the first half of the reporting period, 
Zuari has a generally lower diversity score but finishes 2016 on par with other corporations in the category.

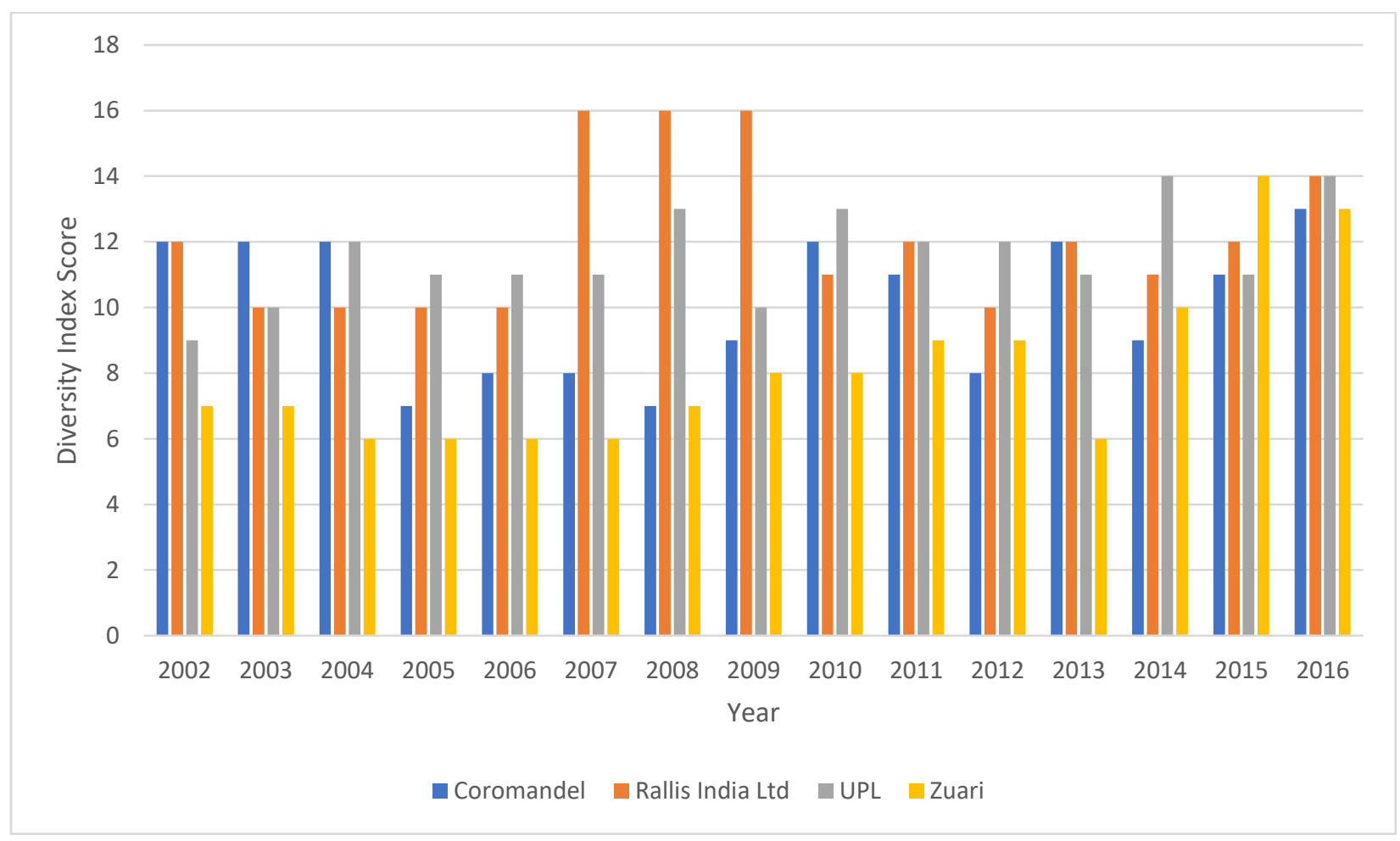

Figure B. 1: Diversity index score for the corporate environmental disclosures from domestic Indian corporations between the years 2002 and 2016. 


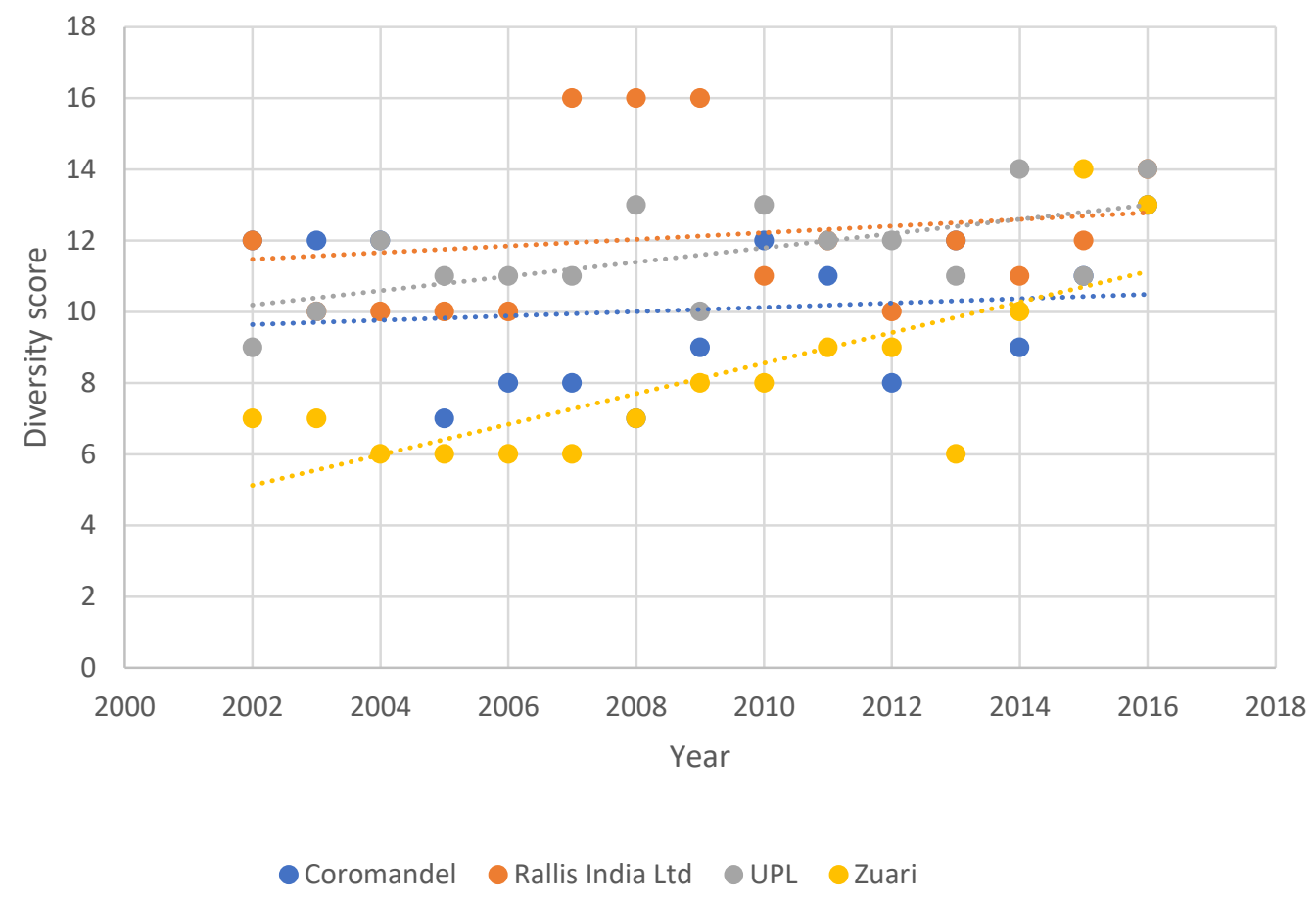

Figure B. 2: Total diversity score of disclosure for domestic Indian corporations from 2002-2016. Coromandel ( $p=0.649221)$, Rallis $(p=0.518678)$, UPL $(p=0.01466)$, Zuari $(p=0.001083)$

Table B. 1: Regression statistics for the diversity of CEDs from Coromandel from 2002-2016

\begin{tabular}{|c|c|c|c|c|c|}
\hline \multicolumn{2}{|c|}{ Regression Statistics } & & & & \\
\hline Multiple R & 0.128065 & & & & \\
\hline $\begin{array}{l}\text { R Square } \\
\text { Adjusted R }\end{array}$ & 0.016401 & & & & \\
\hline Square & -0.05926 & & & & \\
\hline Standard Error & 2.182116 & & & & \\
\hline Observations & 15 & & & & \\
\hline \multicolumn{6}{|l|}{ ANOVA } \\
\hline & $d f$ & SS & MS & $F$ & $\begin{array}{c}\text { Significance } \\
F\end{array}$ \\
\hline Regression & 1 & 1.032143 & 1.032143 & 0.216763 & 0.649221 \\
\hline Residual & 13 & 61.90119 & 4.76163 & & \\
\hline Total & 14 & 62.93333 & & & \\
\hline
\end{tabular}


Table B. 2: Regression statistics for the diversity of CEDs from Rallis from 2002-2016

\begin{tabular}{|c|c|c|c|c|c|}
\hline \multicolumn{2}{|c|}{ Regression Statistics } & & & & \\
\hline Multiple R & 0.180952 & & & & \\
\hline R Square & 0.032743 & & & & \\
\hline Adjusted R & & & & & \\
\hline Square & -0.04166 & & & & \\
\hline Standard Error & 2.342238 & & & & \\
\hline Observations & 15 & & & & \\
\hline \multicolumn{6}{|l|}{ ANOVA } \\
\hline & & & & & Significance \\
\hline & $d f$ & SS & MS & $F$ & $F$ \\
\hline Regression & 1 & 2.414286 & 2.414286 & 0.440075 & 0.518678 \\
\hline Residual & 13 & 71.31905 & 5.486081 & & \\
\hline Total & 14 & 73.73333 & & & \\
\hline
\end{tabular}

Table B. 3: Regression statistics for the diversity of CEDs from UPL from 2002-2016

\begin{tabular}{|c|c|c|c|c|c|}
\hline \multicolumn{2}{|c|}{ Regression Statistics } & & & & \\
\hline Multiple R & 0.615125 & & & & \\
\hline $\begin{array}{l}\text { R Square } \\
\text { Adiusted R }\end{array}$ & 0.378378 & & & & \\
\hline Square & 0.330561 & & & & \\
\hline Standard Error & 1.189699 & & & & \\
\hline Observations & 15 & & & & \\
\hline \multicolumn{6}{|l|}{ ANOVA } \\
\hline & & & & & Significance \\
\hline & $d f$ & SS & MS & $F$ & $F$ \\
\hline Regression & 1 & 11.2 & 11.2 & 7.913043 & 0.01466 \\
\hline Residual & 13 & 18.4 & 1.415385 & & \\
\hline Total & 14 & 29.6 & & & \\
\hline
\end{tabular}


Table B. 4: Regression statistics for the diversity of CEDs from Zuari from 2002-2016

\begin{tabular}{lr}
\hline \multicolumn{2}{c}{ Regression Statistics } \\
\hline Multiple R & 0.757051 \\
R Square & 0.573127 \\
Adjusted R & \\
Square & 0.54029 \\
Standard Error & 1.716543 \\
Observations & 15 \\
\hline
\end{tabular}

\begin{tabular}{|c|c|c|c|c|c|}
\hline & $d f$ & SS & $M S$ & $F$ & $\begin{array}{c}\text { Significance } \\
F\end{array}$ \\
\hline Regression & 1 & 51.42857 & 51.42857 & 17.454 & 0.001083 \\
\hline Residual & 13 & 38.30476 & 2.94652 & & \\
\hline Total & 14 & 89.73333 & & & \\
\hline
\end{tabular}

\subsubsection{Diversity of disclosures from Indian Subsidiaries}

The average diversity of CEDs published by Indian subsidiaries in 2002 was 9.25 out of 18 [Table B.4]. This indicates that out of a possible 18 reporting categories Indian subsidiaries addressed only 9.25 in 2002. By 2016 the average diversity score for Indian subsidiaries had risen to 14.25 [Table B.4]. It should be noted that there were five instances of no publication, Syngenta 2006, 2007, 2012, Bayer 2008 and Monsanto 2014; these instances are not used to calculate the average disclosure for the year. Apart from this their diversity scores are reasonably consistent [Figure B.3]. 


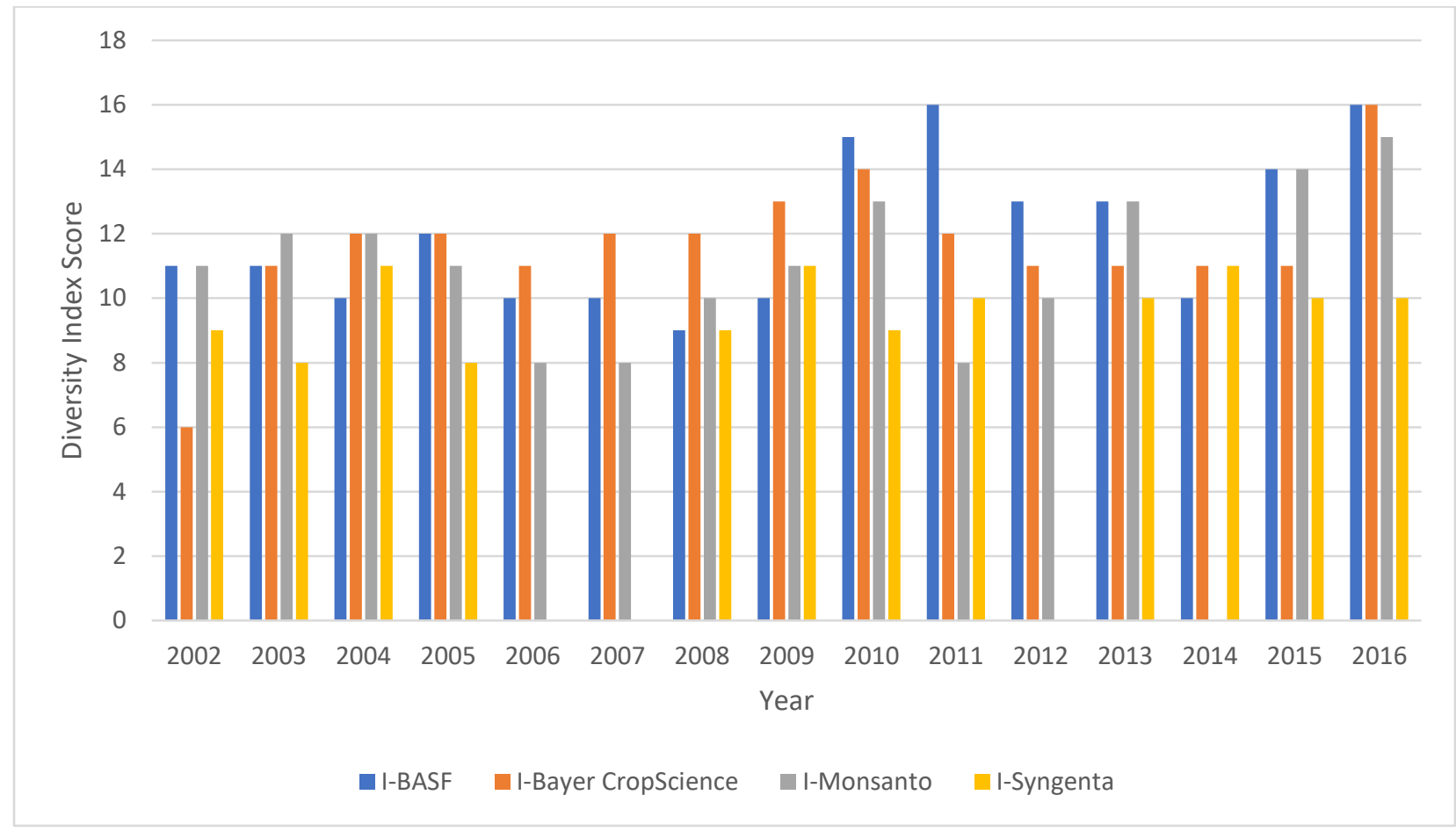

Figure B. 3: Diversity index score for the corporate environmental disclosures from Indian subsidiaries between the years 2002 and 2016.

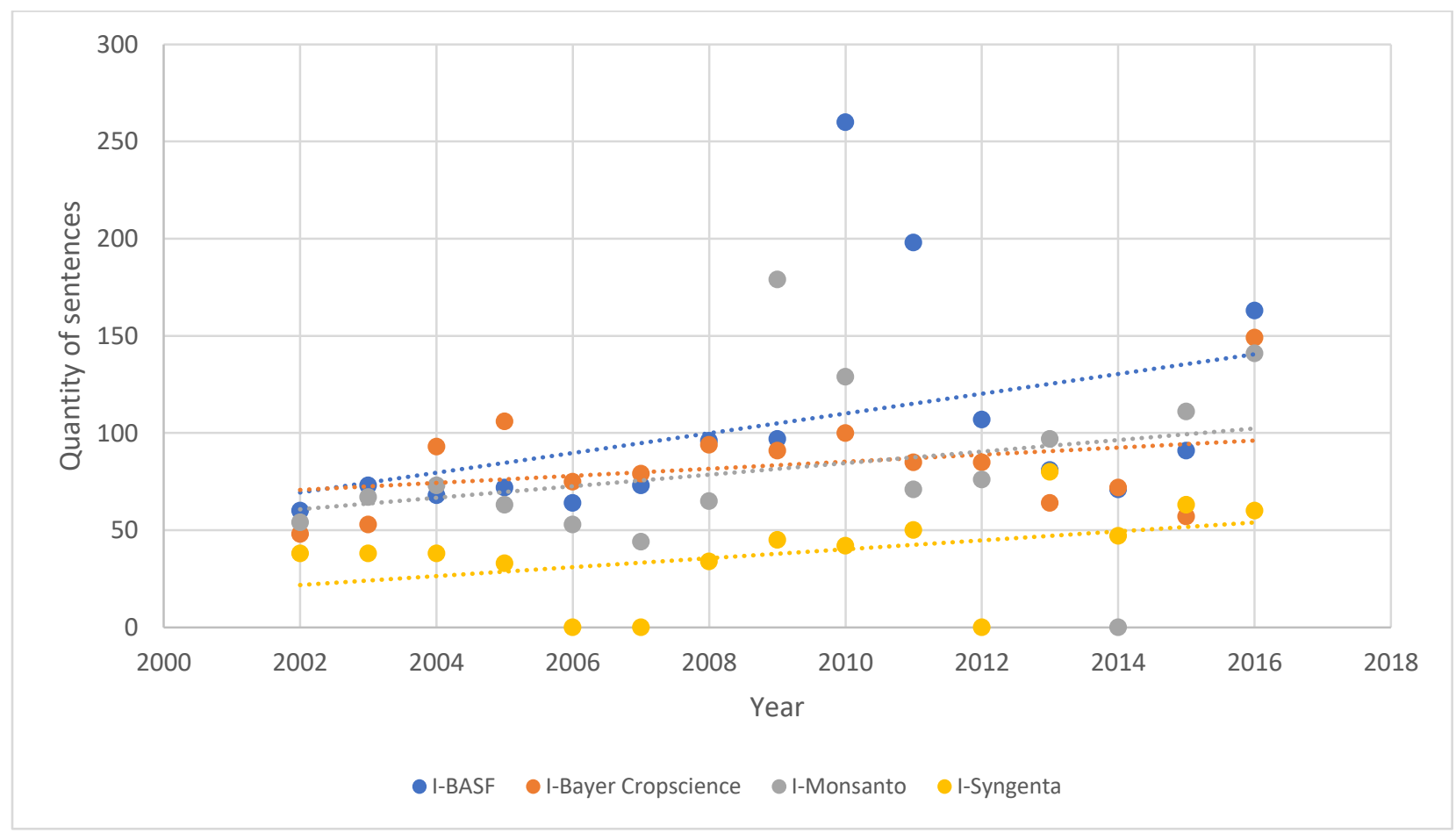

Figure B. 4: Total diversity score of disclosure for I-MNCs from 2002-2016. I-BASF ( $p=0.027049)$, I-Bayer $(p=0.078875)$, I-Monsanto $(p=0.16152)$, I-Syngenta $(p=0.110613)$. 
Table B. 5: Regression statistics for the diversity of CEDs from I-BASF from 2002-2016

\begin{tabular}{|c|c|c|c|c|c|}
\hline \multicolumn{2}{|c|}{ Regression Statistics } & & & & \\
\hline Multiple R & 0.568399 & & & & \\
\hline R Square & 0.323077 & & & & \\
\hline Adjusted R & & & & & \\
\hline Square & 0.271006 & & & & \\
\hline Standard Error & 2.015326 & & & & \\
\hline Observations & 15 & & & & \\
\hline \multicolumn{6}{|l|}{ ANOVA } \\
\hline & & & & & Significance \\
\hline & $d f$ & SS & MS & $F$ & $F$ \\
\hline Regression & 1 & 25.2 & 25.2 & 6.204545 & 0.027049 \\
\hline Residual & 13 & 52.8 & 4.061538 & & \\
\hline Total & 14 & 78 & & & \\
\hline
\end{tabular}

Table B. 6: Regression statistics for the diversity of CEDs from I-Bayer from 2002-2016

\begin{tabular}{|c|c|c|c|c|c|}
\hline \multicolumn{2}{|c|}{ Regression Statistics } & & & & \\
\hline Multiple R & 0.480743 & & & & \\
\hline R Square & 0.231114 & & & & \\
\hline \multicolumn{6}{|l|}{ Adjusted R } \\
\hline Square & 0.171969 & & & & \\
\hline Standard Error & 1.904617 & & & & \\
\hline Observations & 15 & & & & \\
\hline \multicolumn{6}{|l|}{ ANOVA } \\
\hline & & & & & Significance \\
\hline & $d f$ & SS & MS & $F$ & $F$ \\
\hline Regression & 1 & 14.175 & 14.175 & 3.907581 & 0.069676 \\
\hline Residual & 13 & 47.15833 & 3.627564 & & \\
\hline Total & 14 & 61.33333 & & & \\
\hline
\end{tabular}


Table B. 7: Regression statistics for the diversity of CEDs from I-Monsanto from 2002-2016

\begin{tabular}{|c|c|c|c|c|c|}
\hline \multicolumn{2}{|c|}{ Regression Statistics } & & & & \\
\hline Multiple R & 0.395568 & & & & \\
\hline R Square & 0.156474 & & & & \\
\hline Adjusted R & & & & & \\
\hline Square & 0.086181 & & & & \\
\hline Standard Error & 2.116298 & & & & \\
\hline Observations & 14 & & & & \\
\hline \multicolumn{6}{|l|}{ ANOVA } \\
\hline & & & & & Significance \\
\hline & $d f$ & SS & $M S$ & $F$ & $F$ \\
\hline Regression & 1 & 9.969656 & 9.969656 & 2.226006 & 0.16152 \\
\hline Residual & 12 & 53.74463 & 4.478719 & & \\
\hline Total & 13 & 63.71429 & & & \\
\hline
\end{tabular}

Table B. 8: Regression statistics for the diversity of CEDs from I-Syngenta from 2002-2016

\begin{tabular}{|c|c|c|c|c|c|}
\hline \multicolumn{2}{|c|}{ Regression Statistics } & & & & \\
\hline Multiple R & 0.48428 & & & & \\
\hline $\begin{array}{l}\text { R Square } \\
\text { Adjusted R }\end{array}$ & 0.234527 & & & & \\
\hline Square & 0.15798 & & & & \\
\hline Standard Error & 0.984682 & & & & \\
\hline Observations & 12 & & & & \\
\hline \multicolumn{6}{|l|}{ ANOVA } \\
\hline & & & & & Significance \\
\hline & $d f$ & SS & MS & $F$ & $F$ \\
\hline Regression & 1 & 2.970677 & 2.970677 & 3.06382 & 0.110613 \\
\hline Residual & 10 & 9.69599 & 0.969599 & & \\
\hline Total & 11 & 12.66667 & & & \\
\hline
\end{tabular}

\section{B.1.3 Diversity of disclosures from parent multinational corporations}

In 2002 the average diversity score for CEDs from the four selected parent multinational corporations was 11 [Table B.7]. This means that on average these corporations reported in 11 out of a possible 18 categories. By 2016 this average rose to 16.75 [Table B.7], this highest of all the categories of 
corporations. Notably, Bayer CropScience and Monsanto had the lowest diversity scores in 2002 at 6 and 7, respectively [Figure B.5]. BASF and Syngenta had scores of 17 and 14, respectively. However, both Bayer and Monsanto had made discernible improvements by 2005.

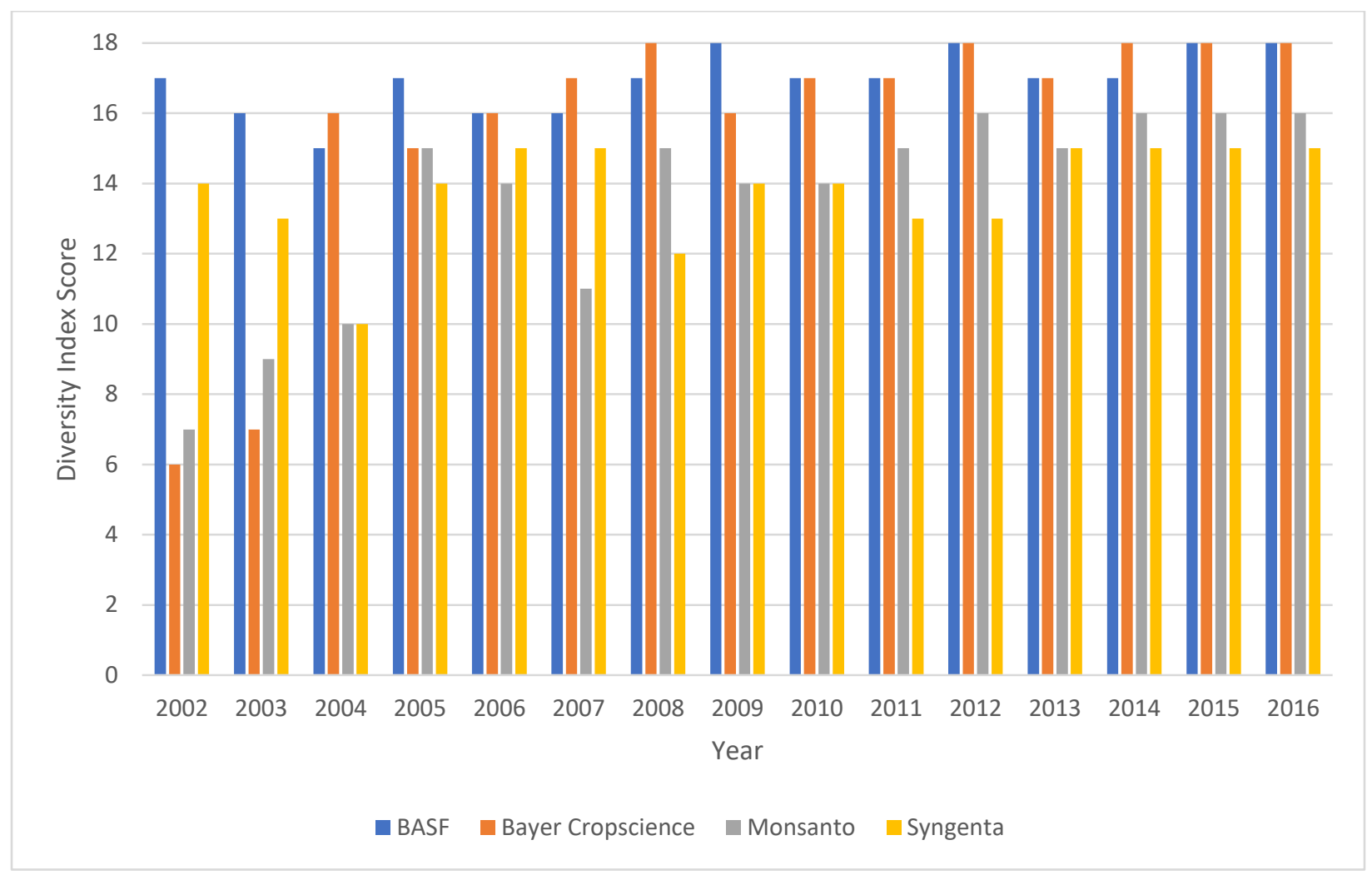

Figure B. 5: Diversity index score for the corporate environmental disclosures from Parent Multinational corporations between the years 2002 and 2016. 


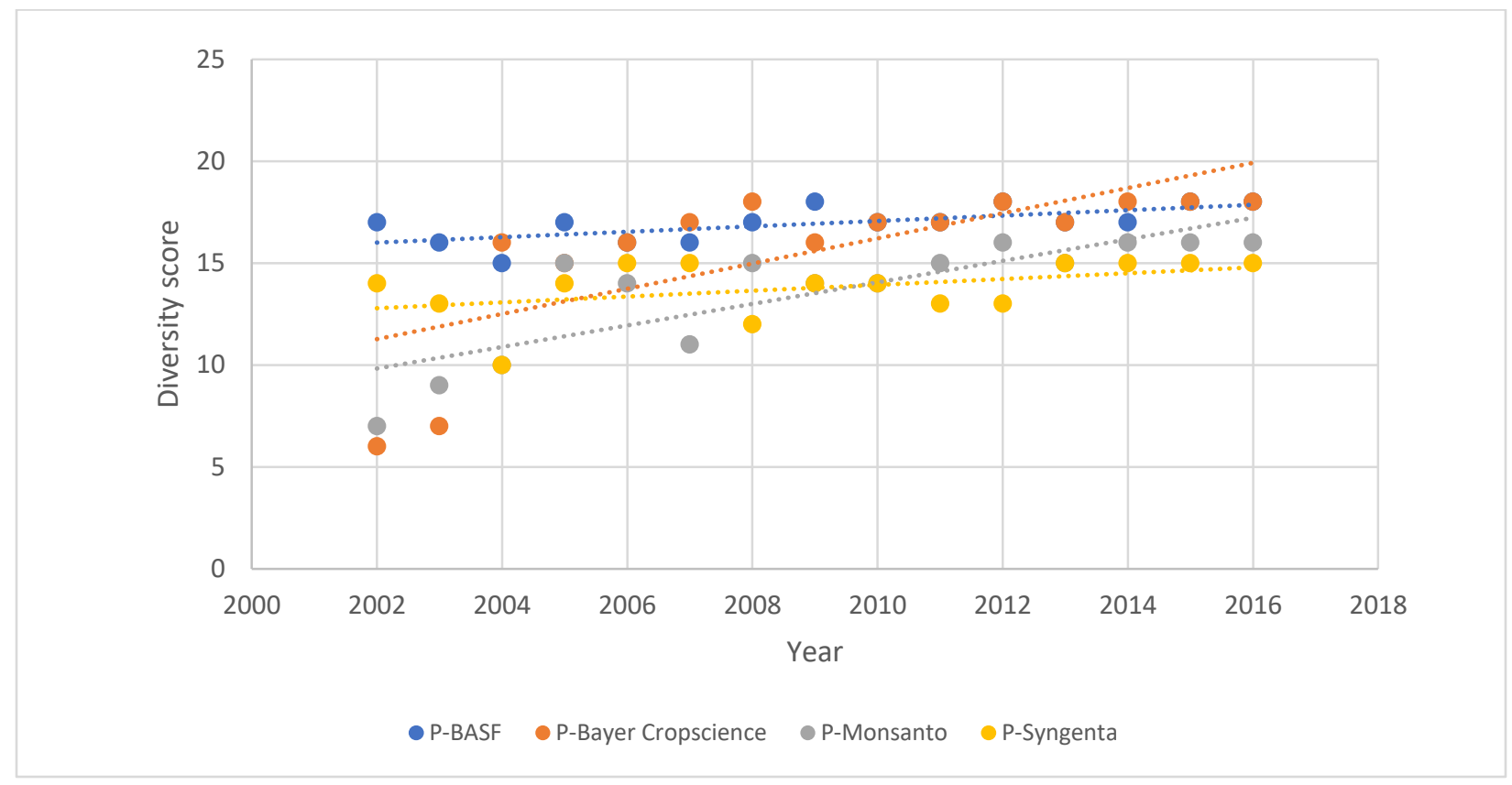

Figure B. 6: Total diversity score of disclosures from 2002-2016. $P$-BASF $(p=0.006416), P$-Bayer $(p=0.002247), P$-Monsanto $(p=0.000169), P$-Syngenta $(p=0.093532)$.

Table B. 9: Regression statistics for the diversity of CEDs from P-BASF from 2002-2016

\begin{tabular}{|c|c|c|c|c|c|}
\hline \multicolumn{2}{|c|}{ Regression Statistics } & & & & \\
\hline Multiple R & 0.668723 & & & & \\
\hline $\begin{array}{l}\text { R Square } \\
\text { Adjusted R }\end{array}$ & 0.447191 & & & & \\
\hline Square & 0.404667 & & & & \\
\hline Standard Error & 0.681855 & & & & \\
\hline Observations & 15 & & & & \\
\hline \multicolumn{6}{|l|}{ ANOVA } \\
\hline & $d f$ & SS & MS & $F$ & $\begin{array}{c}\text { Significance } \\
F \\
\end{array}$ \\
\hline Regression & 1 & 4.889286 & 4.889286 & 10.51625 & 0.006416 \\
\hline Residual & 13 & 6.044048 & 0.464927 & & \\
\hline Total & 14 & 10.93333 & & & \\
\hline
\end{tabular}


Table B. 10: Regression statistics for the diversity of CEDs from P-Bayer from 2002-2016

\begin{tabular}{|c|c|c|c|c|c|}
\hline \multicolumn{2}{|c|}{ Regression Statistics } & & & & \\
\hline Multiple R & 0.724566 & & & & \\
\hline R Square & 0.524996 & & & & \\
\hline Adjusted R & & & & & \\
\hline Square & 0.488458 & & & & \\
\hline Standard Error & 2.727505 & & & & \\
\hline Observations & 15 & & & & \\
\hline \multicolumn{6}{|l|}{ ANOVA } \\
\hline & & & & & Significance \\
\hline & $d f$ & SS & $M S$ & $F$ & $F$ \\
\hline Regression & 1 & 106.8893 & 106.8893 & 14.36822 & 0.002247 \\
\hline Residual & 13 & 96.71071 & 7.439286 & & \\
\hline Total & 14 & 203.6 & & & \\
\hline
\end{tabular}

Table B. 11: Regression statistics for the diversity of CEDs from P-Monsanto from 2002-2016

\begin{tabular}{|c|c|c|c|c|c|}
\hline \multicolumn{2}{|c|}{ Regression Statistics } & & & & \\
\hline Multiple R & 0.822155 & & & & \\
\hline R Square & 0.675938 & & & & \\
\hline Adjusted R & & & & & \\
\hline Square & 0.65101 & & & & \\
\hline Standard Error & 1.698523 & & & & \\
\hline Observations & 15 & & & & \\
\hline \multicolumn{6}{|l|}{ ANOVA } \\
\hline & & & & & Significance \\
\hline & $d f$ & SS & $M S$ & $F$ & $F$ \\
\hline Regression & 1 & 78.22857 & 78.22857 & 27.11579 & 0.000169 \\
\hline Residual & 13 & 37.50476 & 2.884982 & & \\
\hline Total & 14 & 115.7333 & & & \\
\hline
\end{tabular}


Table B. 12: Regression statistics for the diversity of CEDs from P-Syngenta from 2002-2016

\begin{tabular}{lr}
\hline \multicolumn{2}{c}{ Regression Statistics } \\
\hline Multiple R & 0.448561 \\
R Square & 0.201207 \\
Adjusted R & \\
Square & 0.139762 \\
Standard Error & 1.321005 \\
Observations & 15 \\
\hline
\end{tabular}

\begin{tabular}{|c|c|c|c|c|c|}
\hline & & & & & Significance \\
\hline & $d f$ & SS & $M S$ & $F$ & $F$ \\
\hline Regression & 1 & 5.714286 & 5.714286 & 3.274559 & 0.093532 \\
\hline Residual & 13 & 22.68571 & 1.745055 & & \\
\hline Total & 14 & 28.4 & & & \\
\hline
\end{tabular}

\section{B.1.4 Average Diversity for CEDs from all corporations}

By consolidating the results within the categories of corporations it is possible to plot the overall diversity score per categories of corporations per year [Figure B.7]. From this plot, it is possible to see that the overall diversity scores in 2002-2004 were more similar than in later years. A possible reason for this is the missing publication from the Indian subsidiary corporations [Table B.4], as well as the low diversity scores for P-Bayer and P-Monsanto in 2002, 2003 and 2004 [Table B.7]. The most obvious difference in score can be seen in the year 2006-2008 and again in 2012 and 2014. These gaps do lessen slightly in 2010 and again in 2015 and 2016. 


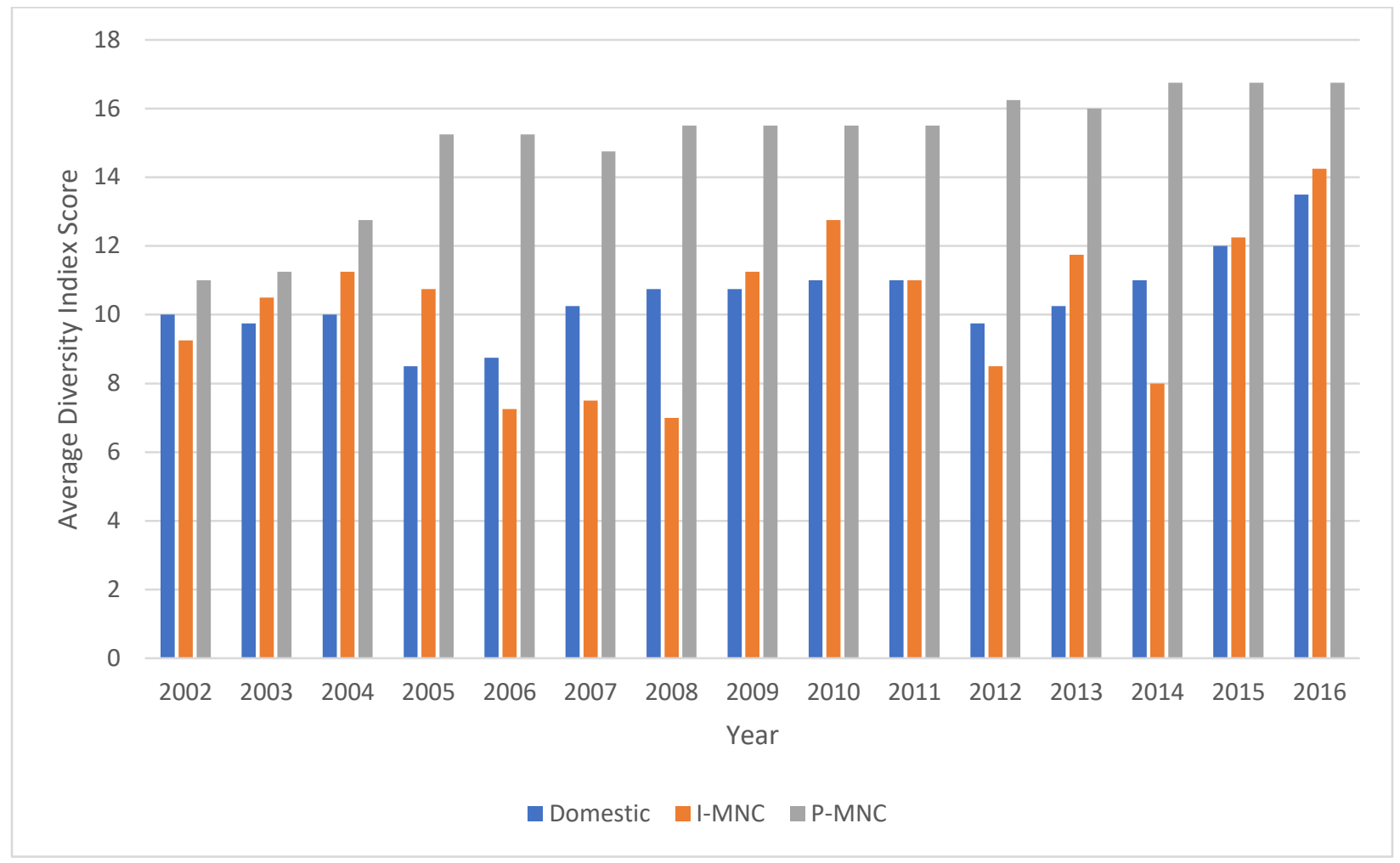

Figure B. 7: Average diversity index score for the corporate environmental disclosures from domestic corporations (Domestic), Indian subsidiary corporations (I-MNC) and parent multinational corporations $(P-M N C)$ between the years 2002 and 2016.

Results of a simple linear regression show that 51 percent of the diversity variation can be attributed to publication year [Table B.2]. Additionally, the diversity of CED reports from domestic Indian corporations in 2016 was statistically significantly higher than in 2002, as determined by ANOVA $(F(1,13)=13.6073, p=0.002728)$ [Table B.3]. Overall, the diversity of CEDs published by domestic Indian corporations seems to be significantly affected by publication year [Table B.3]. 
Table B. 13: Regression statistics for the diversity of CEDs from Domestic Indian Corporations from 20022016

\begin{tabular}{|ll|}
\hline Regression Statistics & \\
\hline Multiple R & 0.7151 \\
R Square & 0.5114 \\
Adjusted R Square & 0.4739 \\
Standard Error & 0.8869 \\
Observations & 15 \\
\hline
\end{tabular}

\begin{tabular}{|llllll|}
\hline ANOVA & Df & SS & MS & F & Significance F \\
\hline Regression & 1 & 10.7056 & 10.7056 & 13.6073 & 0.002728 \\
Residual & 13 & 10.2277 & 0.7867 & & \\
Total & 14 & 20.9332 & & & \\
\hline
\end{tabular}

Results from a simple linear regression show that 46 percent of the variation in diversity of CEDs from Indian subsidiary corporations can be explained by publication year [Figure B.4, Table B.5].

Additionally, the diversity of CED reports from Indian Subsidiary Corporations in 2016 was statistically significantly higher than in 2002, as determined by ANOVA $(F(1,13)=11.3452, p=0.0051)$ [Table B.6]. Based on this it is possible to state that there is a statistically significant difference between the diversity of CEDs from Indian subsidiary corporations in the timeframe 2002-2016. 
Table B. 14: Regression statistics for the diversity of CEDs from Indian Subsidiary Corporations from 20022016

\begin{tabular}{|ll|}
\hline Regression Statistics \\
\hline Multiple R & 0.6825 \\
R Square & 0.4658 \\
Adjusted R Square & 0.4247 \\
Standard Error & 1.0122 \\
Observations & 15 \\
\hline
\end{tabular}

\begin{tabular}{|llllll|}
\hline ANOVA & Df & SS & MS & F & Significance F \\
\hline Regression & 1 & 11.6117 & 11.6117 & 11.33452 & 0.0051 \\
Residual & 13 & 13.3179 & 1.02446 & & \\
Total & 14 & 24.9296 & & & \\
\hline
\end{tabular}

Results from a simple linear regression show that 75 percent of the variation in CED diversity can be attributed to publication year [Figure B.6, Table B.8]. Additionally, the diversity of CED reports from parent multinational corporations in 2016 was statistically significantly higher than in 2002, as determined by ANOVA $(F(1,13)=39.8172, p=0.00002)$ [Table B.9]. Overall, it can be shown that the publication year has a significant effect on the diversity of CED publication from parent multinational corporations. 
Table B. 15: Regression statistics for the diversity of CEDs from Parent Multinational Corporations from 2002-2016

\begin{tabular}{|ll|}
\hline Regression Statistics & \\
\hline Multiple R & 0.8685 \\
R Square & 0.7543 \\
Adjusted R Square & 0.7354 \\
Standard Error & 0.9529 \\
Observations & 15 \\
\hline
\end{tabular}

\begin{tabular}{|llllll|}
\hline ANOVA & Df & SS & MS & F & Significance F \\
\hline Regression & 1 & 36.2520 & 36.2520 & 39.8172 & 0.00002667 \\
Residual & 13 & 11.8063 & 0.9082 & & \\
Total & 14 & 48.0583 & & & \\
\hline
\end{tabular}

\section{B.1.5 Localization or standardization of diversity}

To assess if the diversity of CEDs from I-MNC was more similar to the parent corporations or more localized to the domestic corporations, paired sample t-tests were performed. The T-tests were executed using the data from each corporation for the specified year but is displayed in a table with the category average for reference.

\section{B.1.6 Discussion}

Diversity was used to measure the number of set categories that publication addressed.

Environmental disclosures can cover many different subjects and a well-rounded publication that reports on a variety of issues will rank well for diversity. Categories include everything from disclosure of pollution (POLL), environmental business risk (BRR), and environmental compliance (COMP) to external pressure groups (PRESS) and resource inputs (INP). In total there were 18 possible categories and 59 sub-categories. In this analysis, publications were scored out of the possible 18 categories for reporting. 
At the beginning of the timeframe, in 2002, Domestic corporations were reporting on an average of 10 categories. Meanwhile, I-MNC were averaging 9.25 and P-MNC 11. By 2016, the average diversity of disclosures for domestic corporations was 13.5, which is an increase of 3.5 from 2002. I-MNC also increased, averaging 14.5 by 2016 , which is an increase of 5 from 2002. P-MNC also increased by 5.75 categories to average 16.75 in 2016 . The average diversity for all three categories shows a relatively consistent increase between 2002 and 2016, with P-MNC always having the highest diversity. For the most part, I-MNCs have a slightly higher, but similar level of diversity to domestic corporations. However, between the years 2006-2008, the diversity of disclosures from I-MNC declined slightly and during the same time, the diversity of domestic corporations increased, resulting in an inversion of this trend.

A simple linear regression analysis was performed on all three categories of corporations to determine the significance of the change in diversity score over time. Results from this show that publication year could account for $51 \%$ of diversity variation in domestic corporations, $46 \%$ in I-MNC and 75\% in P-MNC. ANOVA analysis also revealed significant variation in diversity scores for all three groups of corporations. Furthermore, visual inspection of scatter and bar plots all indicate the diversity scores for corporations have an increasing trend. It should be noted that the maximum diversity based on this methodology is 18. Many of the corporations have approached or reached this maximum value; therefore, projections would be required to plateau.

Results from one-tailed two sample paired t-tests were used to assess the standardizations between the diversity scores for the corporations. Comparison between P-MNCs and I-MNCs showed statistically significant differences between the diversity scores for 12 of the years between 2002 and 2016, indicating a lack of standardization. Comparisons between P-MNCs and domestic corporations also showed statistical significance for the same 12 years, again indicating a lack of standardization. Finally, the comparison between I-MNCs and domestic corporations resulted in only one year of 
significance, indicating that the diversity scores for these two categories of corporations were in fact similar.

\section{B.2 CED Quantity}

\section{B.2.1 Quantity of disclosures from domestic Indian corporations}

Coding results for the quantity of disclosure from domestic Indian corporations show a category total of 278 in 2002 and a publication average of 69.5 [Table B.13]. By 2016 the category total had increased to 934 with a publication average of 233.5 [Table B.13]. These measurements were taken from the number of sentences in a publication that had relevant environmental disclosure. This means that in 2002 companies were publishing on average 69.5 sentences containing environmental disclosure and by 2016 were publishing on average 233.5 sentences. Not all corporations published equally, however. UPL had a noticeably low quantity of disclosure in 2002 at 22 sentences but had the highest disclosure in the category by 2016 at 372 sentences [Figure 5.8]. Meanwhile, Coromandel, which had the highest disclosure in 2002 at 101 sentences, was publishing the lowest quality disclosure in 2016 at 192. Also notable is the quantity of disclosure by Rallis India in 2007, 2008 and 2009, here the disclosure quantity spiked to nearly four times what it had been previous years [Table 5.10, Figure 5.8]. 


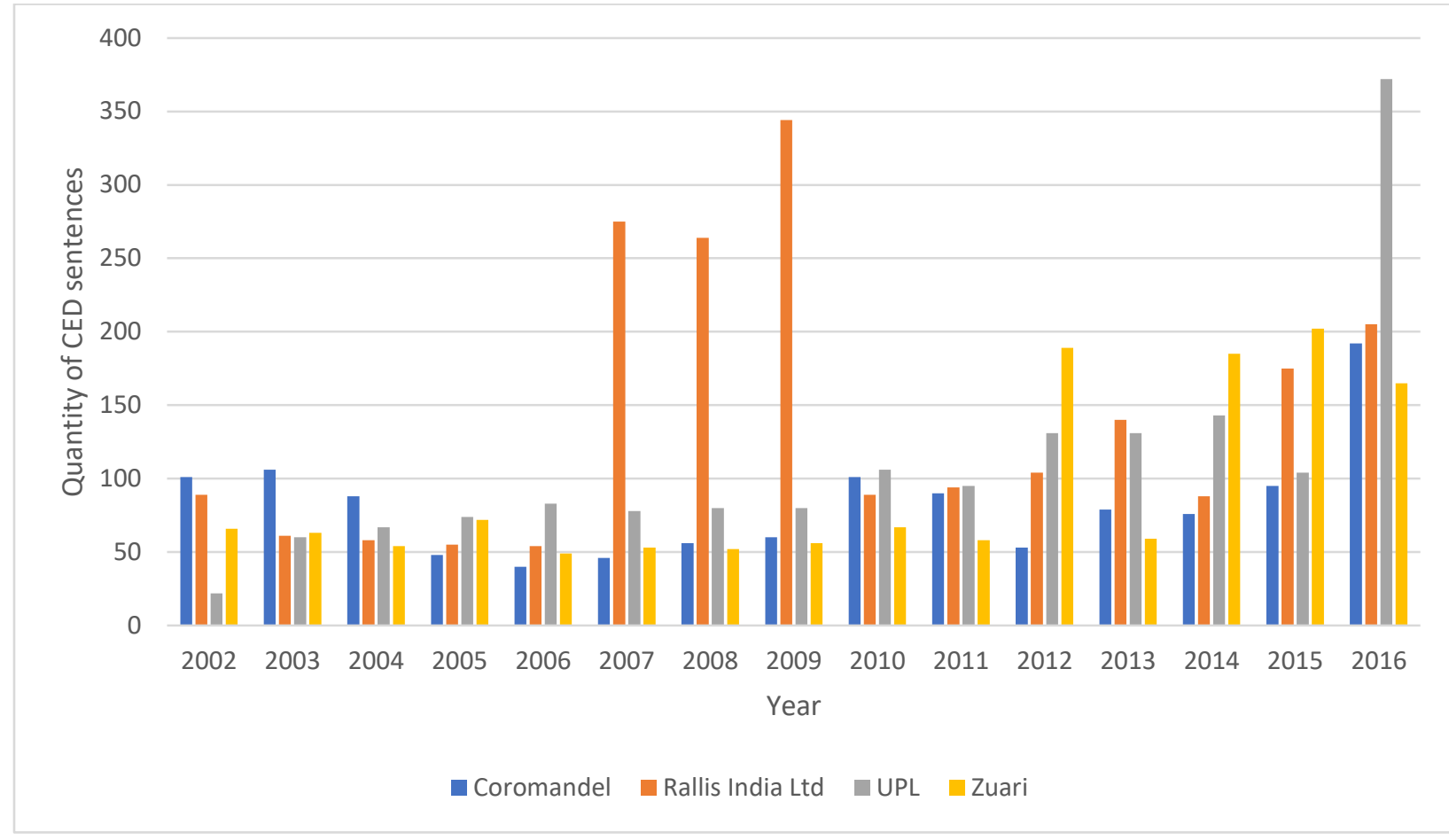

Figure B. 8: Quantity of CED sentences for the corporate environmental disclosures from domestic Indian corporations between the years 2002 and 2016.

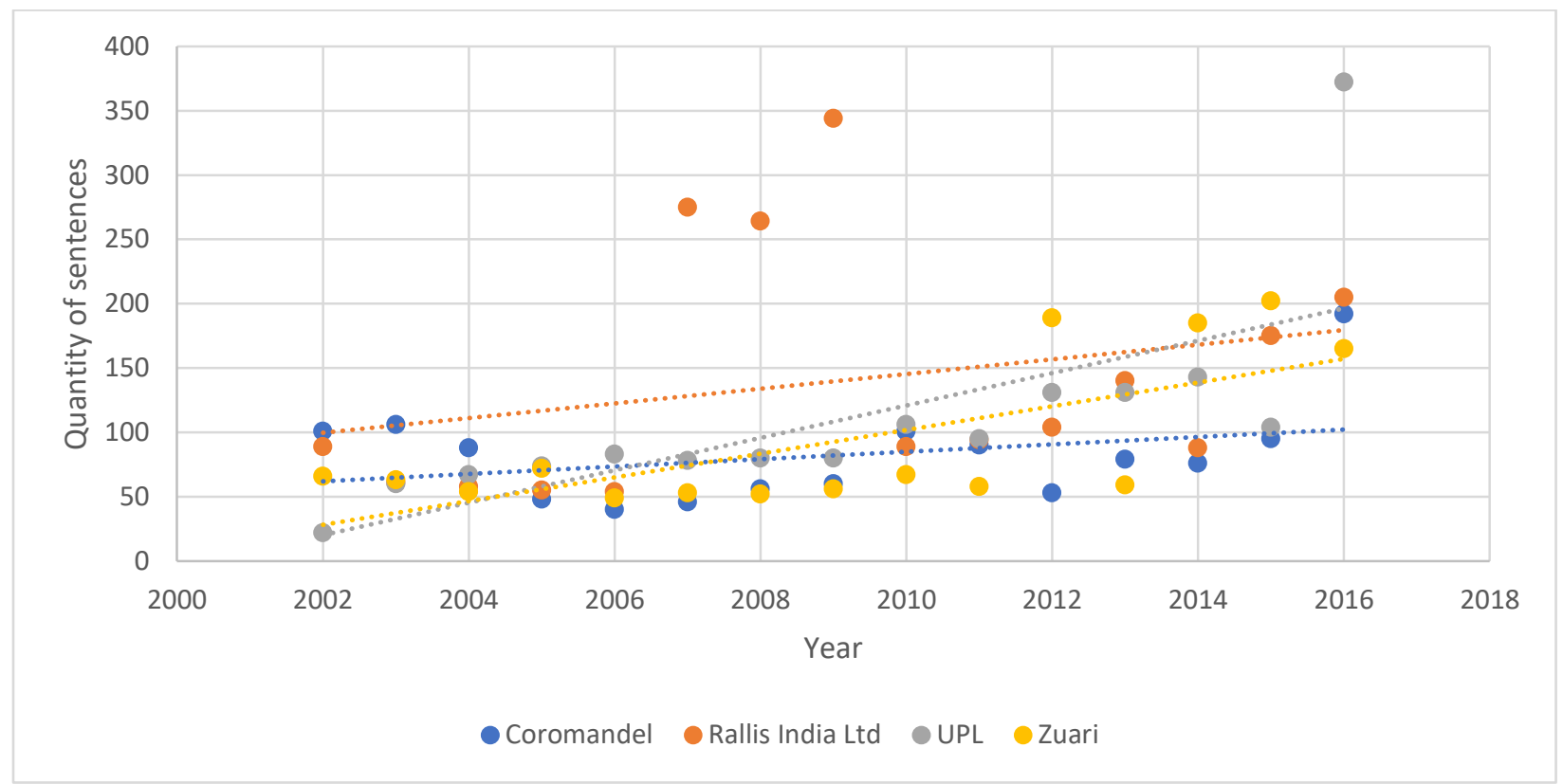

Figure B. 9: Total quantity of disclosures for domestic Indian corporations from 2002-2016. Coromandel $(p=0.212673)$, Rallis ( $p=0.318744)$, UPL $(p=0.002953)$, Zuari $(p=0.003492)$. 
Table B. 16: Regression statistics for the quantity of CEDs from Coromandel from 2002-2016

\begin{tabular}{|c|c|c|c|c|c|}
\hline \multicolumn{2}{|c|}{ Regression Statistics } & & & & \\
\hline Multiple R & 0.341627 & & & & \\
\hline R Square & 0.116709 & & & & \\
\hline $\begin{array}{l}\text { Adjusted R } \\
\text { Square }\end{array}$ & 0.048764 & & & & \\
\hline Standard Error & 36.79783 & & & & \\
\hline Observations & 15 & & & & \\
\hline \multicolumn{6}{|l|}{ ANOVA } \\
\hline & & & & & Significance \\
\hline & $d f$ & SS & MS & $F$ & $F$ \\
\hline Regression & 1 & 2325.889 & 2325.889 & 1.717689 & 0.212673 \\
\hline Residual & 13 & 17603.04 & 1354.08 & & \\
\hline Total & 14 & 19928.93 & & & \\
\hline
\end{tabular}

Table B. 17: Regression statistics for the quantity of CEDs from Rallis from 2002-2016

\begin{tabular}{|c|c|c|c|c|c|}
\hline \multicolumn{2}{|c|}{ Regression Statistics } & & & & \\
\hline Multiple R & 0.27635 & & & & \\
\hline $\begin{array}{l}\text { R Square } \\
\text { Adjusted R }\end{array}$ & 0.076369 & & & & \\
\hline Square & 0.005321 & & & & \\
\hline Standard Error & 92.16953 & & & & \\
\hline Observations & 15 & & & & \\
\hline \multicolumn{6}{|l|}{ ANOVA } \\
\hline & $d f$ & SS & MS & $F$ & $\begin{array}{c}\text { Significance } \\
F\end{array}$ \\
\hline Regression & 1 & 9131.432 & 9131.432 & 1.07489 & 0.318744 \\
\hline Residual & 13 & 110437.9 & 8495.223 & & \\
\hline Total & 14 & 119569.3 & & & \\
\hline
\end{tabular}


Table B. 18: Regression statistics for the quantity of CEDs from UPL from 2002-2016

\begin{tabular}{|c|c|c|c|c|c|}
\hline \multicolumn{2}{|c|}{ Regression Statistics } & & & & \\
\hline Multiple R & 0.711159 & & & & \\
\hline R Square & 0.505748 & & & & \\
\hline Adjusted R & & & & & \\
\hline Square & 0.467728 & & & & \\
\hline Standard Error & 57.77489 & & & & \\
\hline Observations & 15 & & & & \\
\hline \multicolumn{6}{|l|}{ ANOVA } \\
\hline & & & & & Significance \\
\hline & $d f$ & SS & $M S$ & $F$ & $F$ \\
\hline Regression & 1 & 44402.41 & 44402.41 & 13.30235 & 0.002953 \\
\hline Residual & 13 & 43393.19 & 3337.937 & & \\
\hline Total & 14 & 87795.6 & & & \\
\hline
\end{tabular}

Table B. 19: Regression statistics for the quantity of CEDs from Zuari from 2002-2016

\begin{tabular}{|c|c|c|c|c|c|}
\hline \multicolumn{2}{|c|}{ Regression Statistics } & & & & \\
\hline Multiple R & 0.702555 & & & & \\
\hline $\begin{array}{l}\text { R Square } \\
\text { Adjusted R }\end{array}$ & 0.493583 & & & & \\
\hline Square & 0.454628 & & & & \\
\hline Standard Error & 43.23151 & & & & \\
\hline Observations & 15 & & & & \\
\hline \multicolumn{6}{|l|}{ ANOVA } \\
\hline & $d f$ & SS & MS & $F$ & $\begin{array}{c}\text { Significance } \\
F\end{array}$ \\
\hline Regression & 1 & 23680.8 & 23680.8 & 12.67055 & 0.003492 \\
\hline Residual & 13 & 24296.53 & 1868.964 & & \\
\hline Total & 14 & 47977.33 & & & \\
\hline
\end{tabular}

\subsubsection{Quantity of disclosures from Indian subsidiaries}

Coding results for the quantity of CED disclosure in publications by Indian subsidiary corporations show a total quantity of 200 in 2002, with a publication average of 50 [Table B.16]. Both the total quantity and average quantity increased by 2016 where the total quantity reported was 513 and the publication average was 128.25 [Table B.16]. Just as in the previous section, quantity is 
measured by the number of sentences published by the corporation that include relevant environmental disclosures. Therefore, these results indicate that the average number of CED sentences per publication rose from 50 in 2002 to 128.25 in 2016. Most corporations show a relatively consistent increase in quantity over the timeframe. However, Monsanto's quantity noticeably spiked in 2009 and remained high in 2010 before dropping back down [Figure B.13].

It should also be noted that several corporations in this category did not publish reports in certain years, those being Syngenta in 2006, 2007 and 2012, as well as Monsanto in 2014. These were not included as data points when calculating the year average.

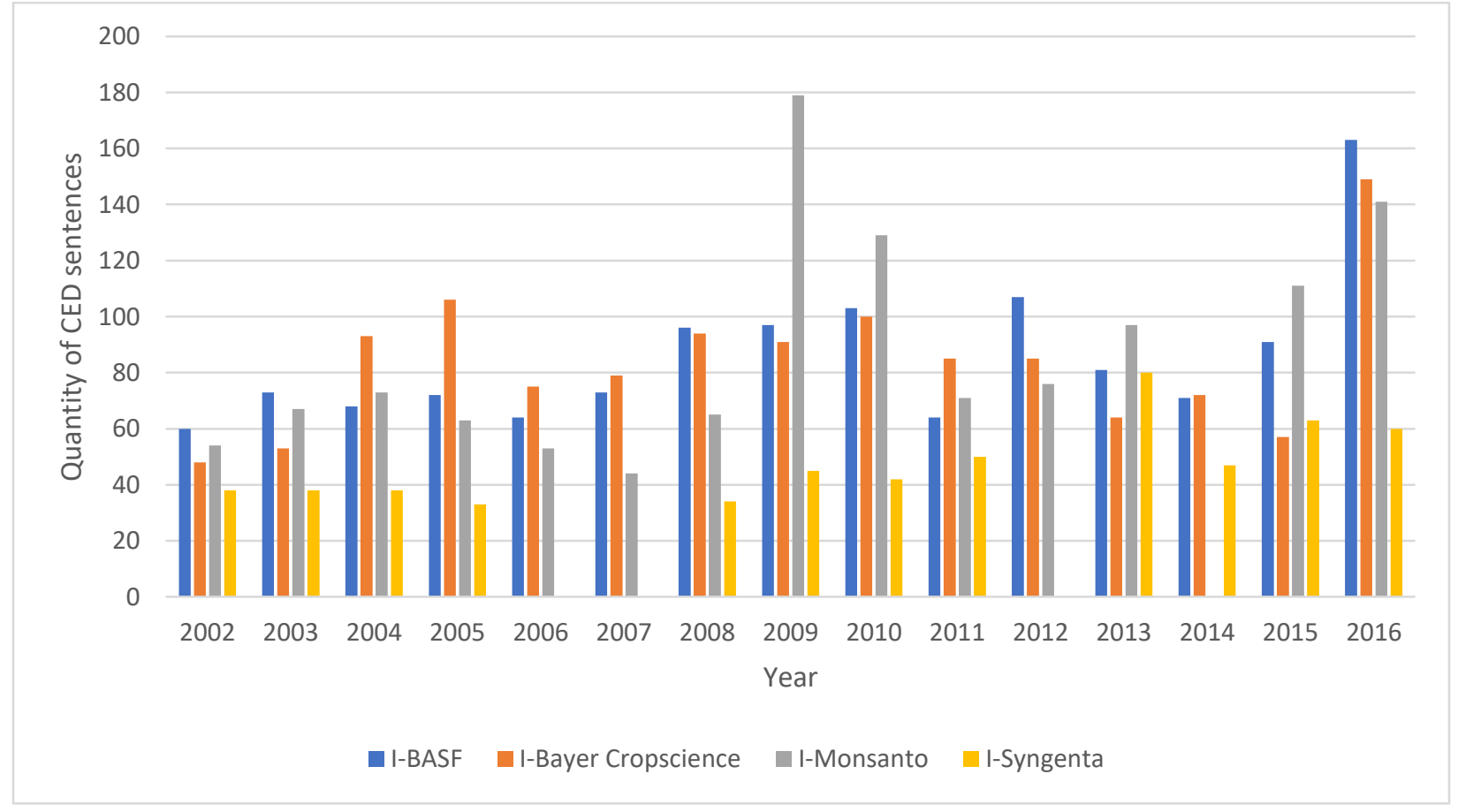

Figure B. 10: Quantity of CED sentences for the corporate environmental disclosures from Indian subsidiaries between the years 2002 and 2016. 


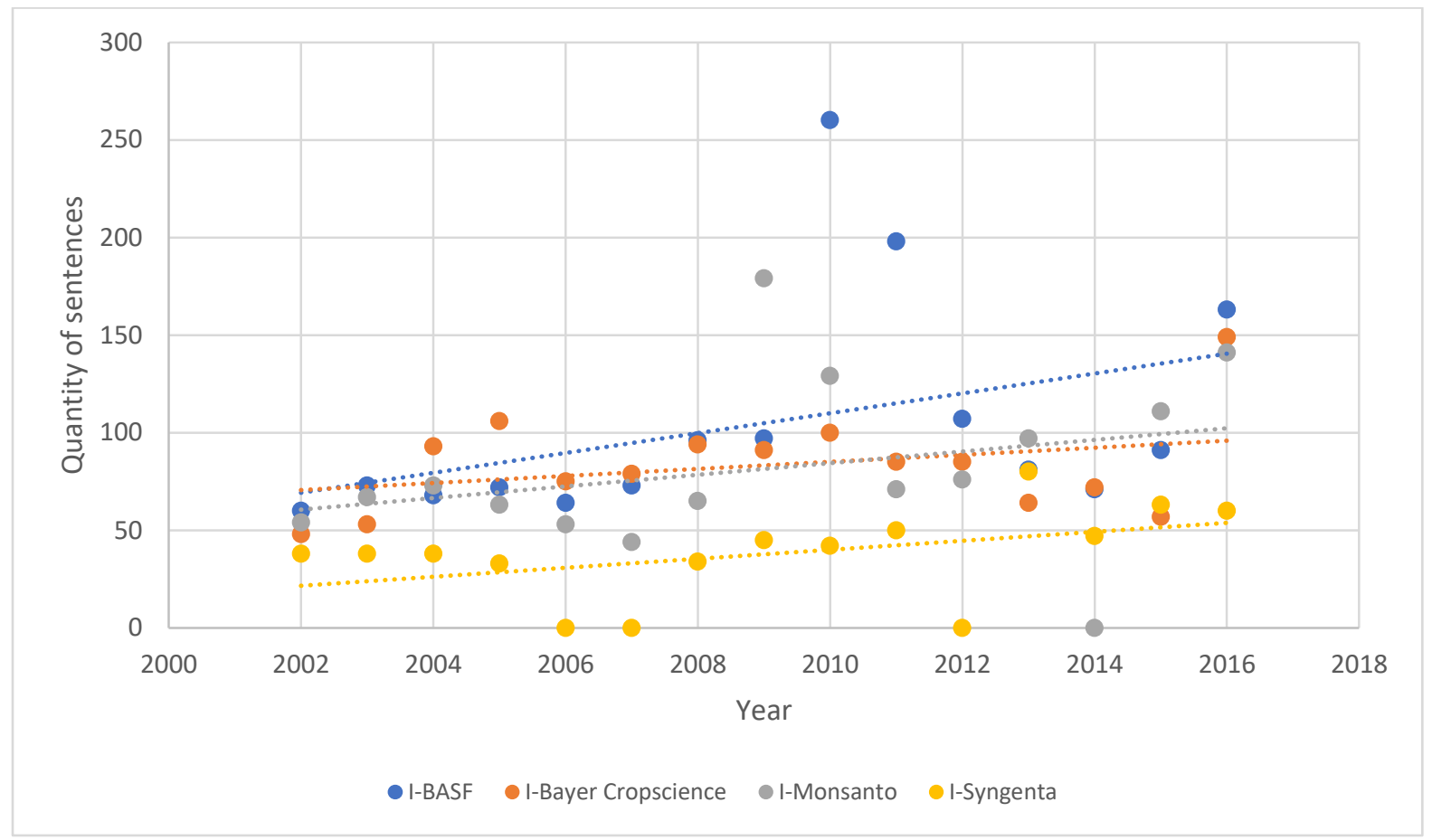

Figure B. 11: Total quantity of disclosure for I-MNCs from 2002-2016. I-BASF ( $p=0.144962)$, I-Bayer $(p=0.242071)$, I-Monsanto $(p=0.274214)$, I-Syngenta $(p=0.097297)$.

Table B. 20: Regression statistics for the quantity of CEDs from I-BASF from 2002-2016

\begin{tabular}{|c|c|c|c|c|c|}
\hline \multicolumn{2}{|c|}{ Regression Statistics } & & & & \\
\hline Multiple R & 0.607021 & & & & \\
\hline R Square & 0.368475 & & & & \\
\hline Adjusted R & & & & & \\
\hline Square & 0.319896 & & & & \\
\hline Standard Error & 21.65566 & & & & \\
\hline Observations & 15 & & & & \\
\hline \multicolumn{6}{|l|}{ ANOVA } \\
\hline & & & & & Significance \\
\hline & $d f$ & SS & MS & $F$ & $F$ \\
\hline Regression & 1 & 3557.157 & 3557.157 & 7.585084 & 0.016409 \\
\hline Residual & 13 & 6096.576 & 468.9674 & & \\
\hline Total & 14 & 9653.733 & & & \\
\hline
\end{tabular}

Table B. 21: Regression statistics for the quantity of CEDs from I-Bayer from 2002-2016 


\begin{tabular}{|c|c|c|c|c|c|}
\hline \multicolumn{2}{|c|}{ Regression Statistics } & & & & \\
\hline Multiple R & 0.395102 & & & & \\
\hline $\begin{array}{l}\text { R Square } \\
\text { Adjusted R }\end{array}$ & 0.156106 & & & & \\
\hline Square & 0.091191 & & & & \\
\hline Standard Error & 54.83896 & & & & \\
\hline Observations & 15 & & & & \\
\hline \multicolumn{6}{|l|}{ ANOVA } \\
\hline & & & & & Significance \\
\hline & $d f$ & SS & MS & $F$ & $F$ \\
\hline Regression & 1 & 7231.889 & 7231.889 & 2.404769 & 0.144962 \\
\hline Residual & 13 & 39095.04 & 3007.311 & & \\
\hline Total & 14 & 46326.93 & & & \\
\hline
\end{tabular}

Table B. 22: Regression statistics for the quantity of CEDs from I-Monsanto from 2002-2016

\begin{tabular}{|c|c|c|c|c|c|}
\hline \multicolumn{2}{|c|}{ Regression Statistics } & & & & \\
\hline Multiple R & 0.301853 & & & & \\
\hline $\begin{array}{l}\text { R Square } \\
\text { Adjusted R }\end{array}$ & 0.091115 & & & & \\
\hline Square & 0.021201 & & & & \\
\hline Standard Error & 43.50194 & & & & \\
\hline Observations & 15 & & & & \\
\hline \multicolumn{6}{|l|}{ ANOVA } \\
\hline & & & & & Significance \\
\hline & $d f$ & SS & $M S$ & $F$ & $F$ \\
\hline Regression & 1 & 2466.289 & 2466.289 & 1.303247 & 0.274214 \\
\hline Residual & 13 & 24601.44 & 1892.419 & & \\
\hline Total & 14 & 27067.73 & & & \\
\hline
\end{tabular}

Table B. 23: Regression statistics for the quantity of CEDs from I-Syngenta from 2002-2016 


\begin{tabular}{|c|c|c|c|c|c|}
\hline \multicolumn{2}{|c|}{ Regression Statistics } & & & & \\
\hline Multiple R & 0.444036 & & & & \\
\hline $\begin{array}{l}\text { R Square } \\
\text { Adjusted R }\end{array}$ & 0.197168 & & & & \\
\hline Square & 0.135411 & & & & \\
\hline Standard Error & 21.57265 & & & & \\
\hline Observations & 15 & & & & \\
\hline \multicolumn{6}{|l|}{ ANOVA } \\
\hline & & & & & Significance \\
\hline & $d f$ & SS & $M S$ & $F$ & $F$ \\
\hline Regression & 1 & 1485.804 & 1485.804 & 3.192673 & 0.097297 \\
\hline Residual & 13 & 6049.93 & 465.3792 & & \\
\hline Total & 14 & 7535.733 & & & \\
\hline
\end{tabular}

\subsubsection{Quantity of disclosures from parent multinational corporations}

The quantity of CED disclosure for publications by parent multinational corporations totalled 921 in 2002, with a publication average of 230.25 [Table B.19]. By 2016 the total quantity of CEDs for the category was 2308 , with a publication average of 577 [Table B.19]. BASF was the most consistent reporting company for quantity over the entire timeframe, reporting 589 CED sentences in 2002 and 615 sentences in 2016. Bayer was quite noticeably the least consistent; it reported 43 CED sentences in 2002, quickly spiked to 1141 two years later in 2004 and ended 2016 with 599 CED sentences [Figure B.12]. This can be partially attributed to Bayer's occasional publication of a standalone report. 


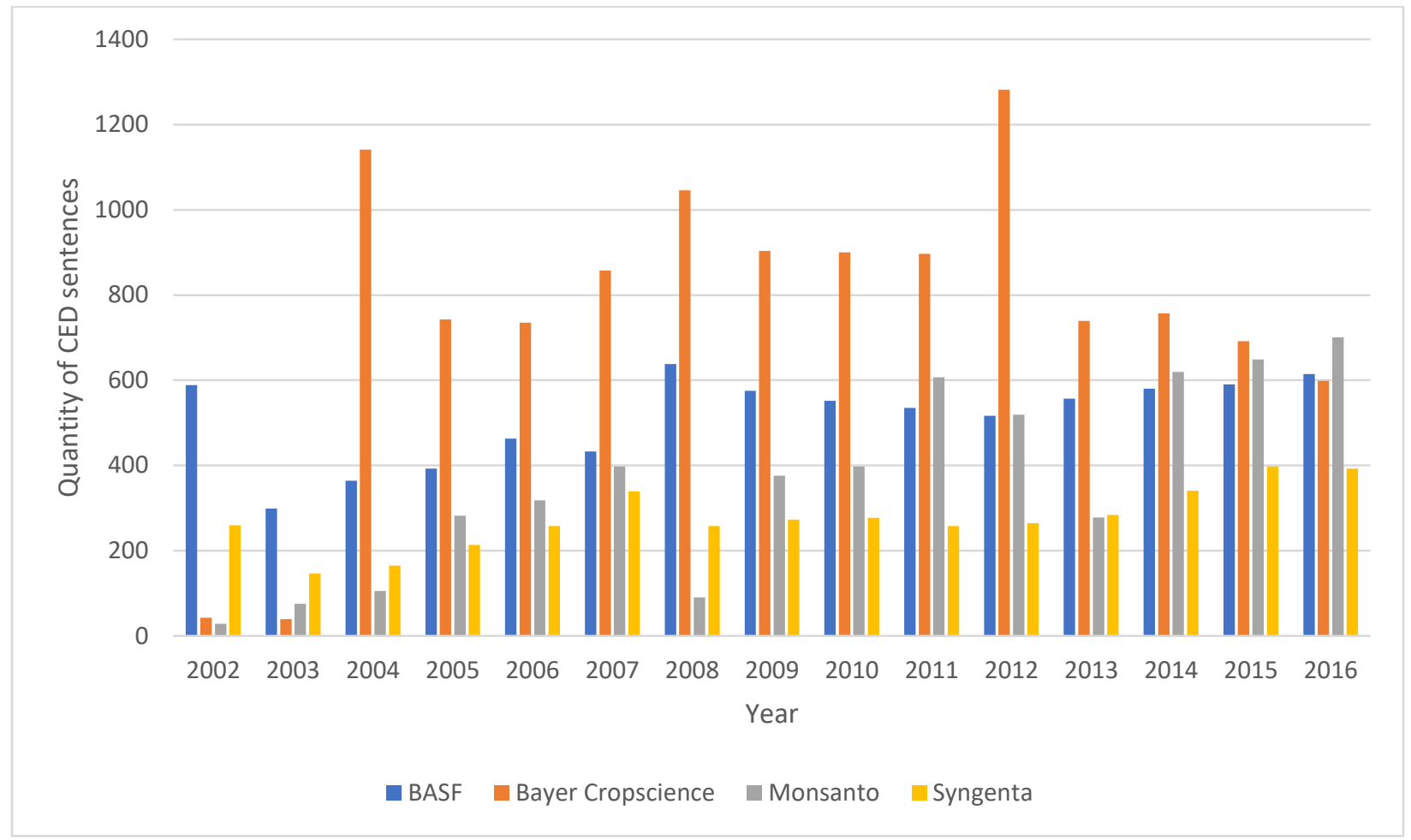

Figure B. 12: Quantity of CED sentences for the corporate environmental disclosures from parent multinational corporations between the years 2002 and 2016

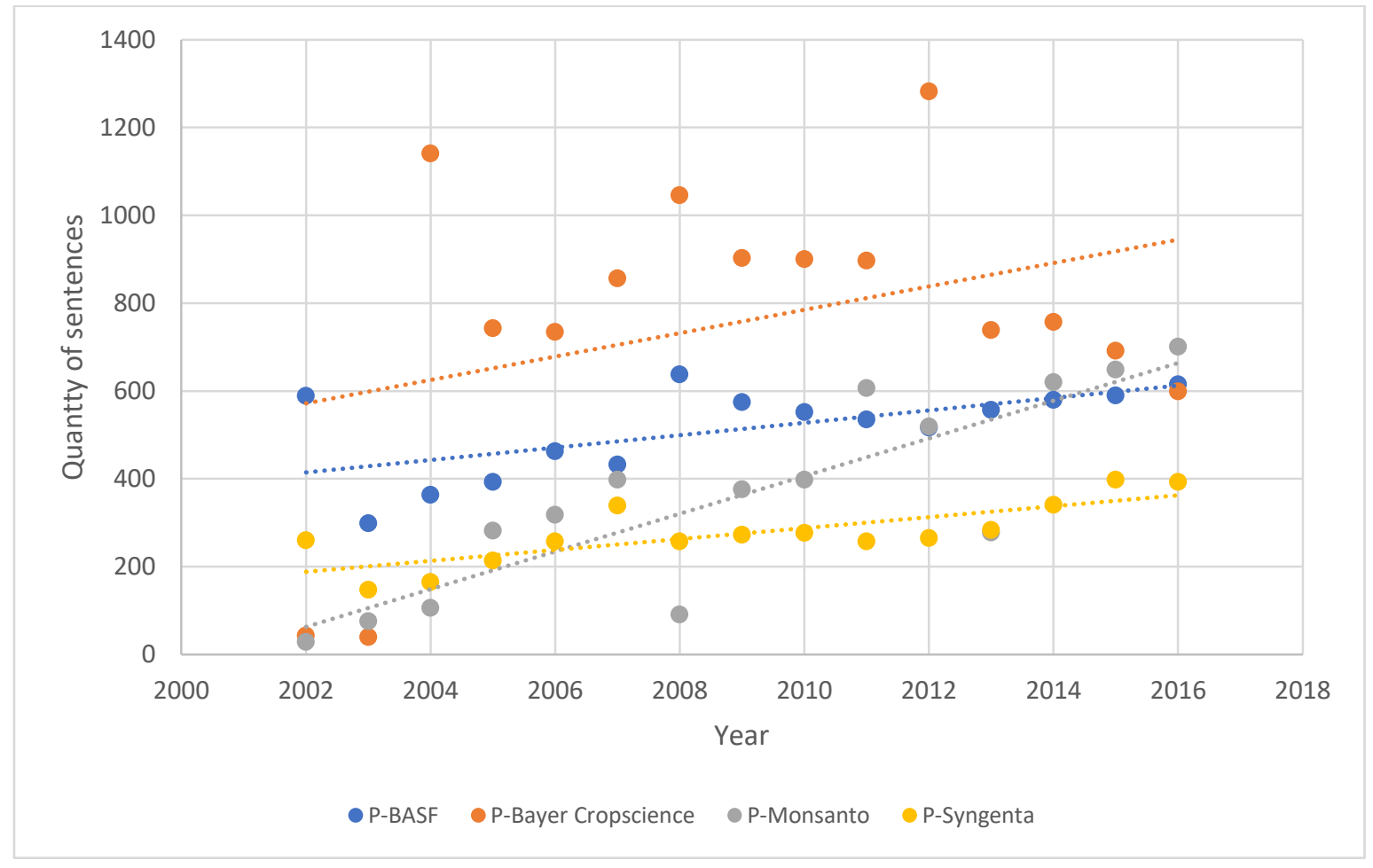

Figure B. 13: Total quantity of disclosure for $P$-MNCs from 2002-2016. $P$-BASF $(p=0.012364), P$-Bayer $(p=0.203456)$, P-Monsanto ( $p=3.94919 E-05)$, P-Syngenta $(p=0.000613)$. 
Table B. 24: Regression statistics for the quantity of CEDs from P-BASF from 2002-2016

\begin{tabular}{|c|c|c|c|c|c|}
\hline \multicolumn{2}{|c|}{ Regression Statistics } & & & & \\
\hline Multiple R & 0.626984927 & & & & \\
\hline R Square & 0.393110099 & & & & \\
\hline Adjusted R & & & & & \\
\hline Square & 0.346426261 & & & & \\
\hline Standard Error & 81.22394313 & & & & \\
\hline Observations & 15 & & & & \\
\hline \multicolumn{6}{|l|}{ ANOVA } \\
\hline & & & & & Significance \\
\hline & $d f$ & SS & $M S$ & $F$ & $F$ \\
\hline Regression & 1 & 55554.06 & 55554.06 & 8.420689 & 0.012364 \\
\hline Residual & 13 & 85765.28 & 6597.329 & & \\
\hline Total & 14 & 141319.3 & & & \\
\hline
\end{tabular}

Table B. 25: Regression statistics for the quantity of CEDs from P-Bayer from 2002-2016

\begin{tabular}{|c|c|c|c|c|c|}
\hline \multicolumn{2}{|c|}{ Regression Statistics } & & & & \\
\hline Multiple R & 0.348182 & & & & \\
\hline R Square & 0.121231 & & & & \\
\hline $\begin{array}{l}\text { Adjusted R } \\
\text { Square }\end{array}$ & 0.053633 & & & & \\
\hline Standard Error & 332.1457 & & & & \\
\hline Observations & 15 & & & & \\
\hline \multicolumn{6}{|l|}{ ANOVA } \\
\hline & $d f$ & SS & MS & $F$ & $\begin{array}{c}\text { Significance } \\
F\end{array}$ \\
\hline Regression & 1 & 197850.9 & 197850.9 & 1.793415 & 0.203456 \\
\hline Residual & 13 & 1434170 & 110320.8 & & \\
\hline Total & 14 & 1632021 & & & \\
\hline
\end{tabular}

Table B. 26: Regression statistics for the quantity of CEDs from P-Monsanto from 2002-2016 


\begin{tabular}{|c|c|c|c|c|c|}
\hline \multicolumn{2}{|c|}{ Regression Statistics } & & & & \\
\hline Multiple R & 0.859872 & & & & \\
\hline $\begin{array}{l}\text { R Square } \\
\text { Adjusted R }\end{array}$ & 0.73938 & & & & \\
\hline Square & 0.719332 & & & & \\
\hline Standard Error & 118.3227 & & & & \\
\hline Observations & 15 & & & & \\
\hline \multicolumn{6}{|l|}{ ANOVA } \\
\hline & & & & & Significance \\
\hline & $d f$ & SS & $M S$ & $F$ & $F$ \\
\hline Regression & 1 & 516344.9 & 516344.9 & 36.88107324 & $3.95 \mathrm{E}-05$ \\
\hline Residual & 13 & 182003.5 & 14000.27 & & \\
\hline Total & 14 & 698348.4 & & & \\
\hline
\end{tabular}

Table B. 27: Regression statistics for the quantity of CEDs from P-Syngenta from 2002-2016

\begin{tabular}{lr}
\hline \multicolumn{2}{c}{ Regression Statistics } \\
\hline Multiple R & 0.779434 \\
R Square & 0.607517 \\
Adjusted R & \\
Square & 0.577326 \\
Standard Error & 46.29512 \\
Observations & 15 \\
\hline
\end{tabular}

ANOVA

\begin{tabular}{lrrrcc}
\hline & & & & \multicolumn{2}{c}{ Significance } \\
& $d f$ & \multicolumn{1}{c}{ SS } & MS & \multicolumn{1}{c}{$F$} & \multicolumn{1}{c}{$F$} \\
\hline Regression & 1 & 43127.23 & 43127.23 & 20.12246 & 0.000613 \\
Residual & 13 & 27862.1 & 2143.239 & & \\
Total & 14 & 70989.33 & & & \\
\hline
\end{tabular}

\subsubsection{Total quantity of disclosures from all corporations}

Finally, results were consolidated and plotted to visually display the total quantity of each category of the corporation for the timeframe [Figure B.14]. From this plot, it can be seen that all categories increased CED quantity between 2002 and 2016 at a relatively consistent pace. The quantity of CEDs published by P-MNC is notably higher than any of the other categories. Domestic corporations also maintain a higher or similar quantity of CED sentences than I-MNC. This suggests that in terms of 
quantity I-MNC publish reports that are more similar to domestic reports as opposed to more similar to P-MNC.

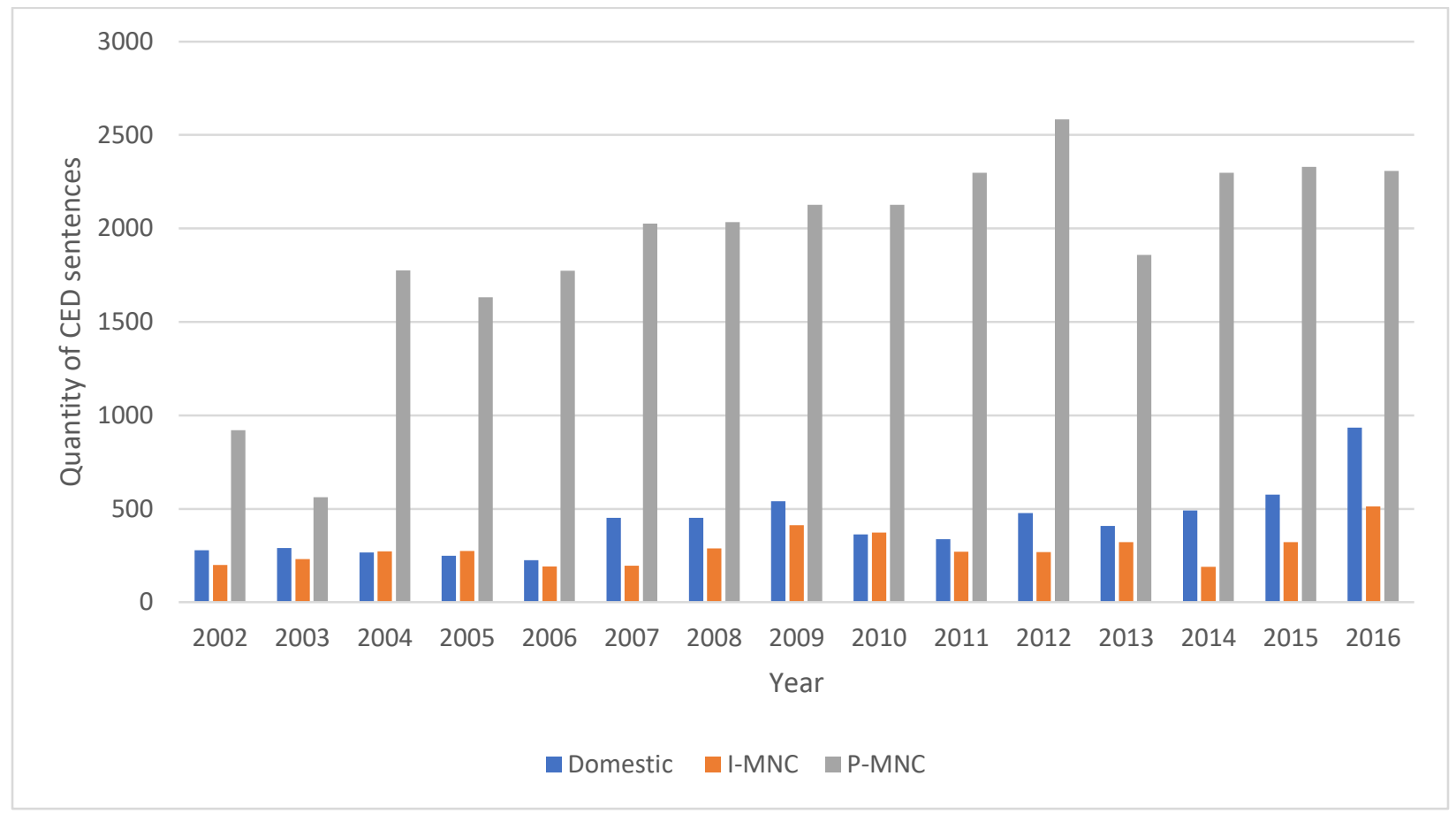

Figure B. 14: Total quantity of CED sentences for the corporate environmental disclosures from all companies in the category Domestic Indian corporations (Domestic), Indian subsidiary corporations (IMNC) and parents multinational corporations (P-MNC) between the years 2002 and 2016. 


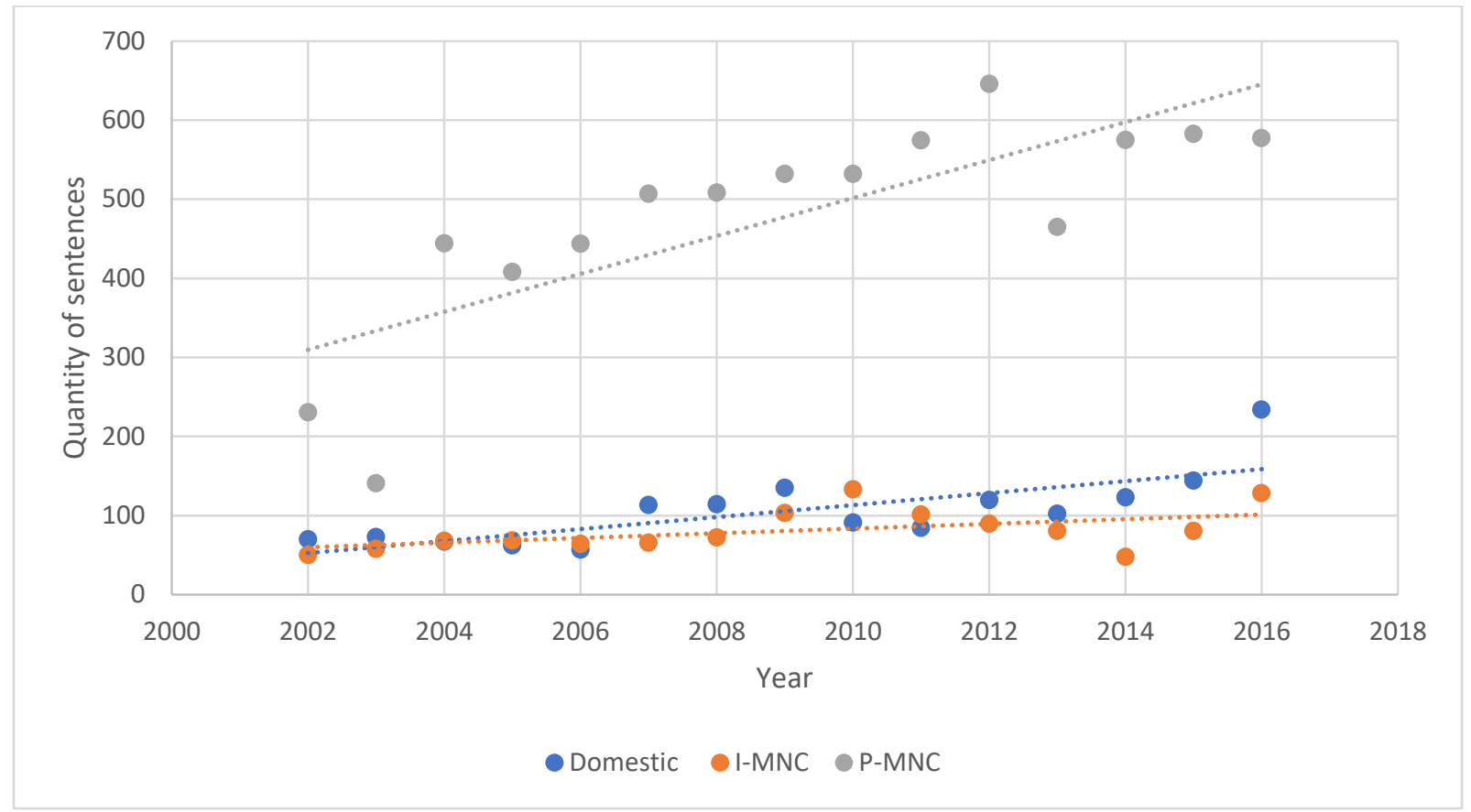

Figure B. 15: Mean quantity of disclosure for domestic Indian corporations, I-MNCs and P-MNC for 20022016. Domestic $(p=0.001097), I-M N C(p=0.049118), P-M N C(p=0.00044)$.

Table B. 28: Regression statistics for the quantity of CEDs from domestic Indian corporations from 20022016

\begin{tabular}{|ll|}
\hline Regression Statistics \\
\hline Multiple R & 0.7571 \\
R Square & 0.5732 \\
Adjusted R Square & 0.5404 \\
Standard Error & 30.4172 \\
Observations & 15 \\
\hline
\end{tabular}

\begin{tabular}{|llllll|}
\hline ANOVA & Df & SS & MS & F & Significance F \\
\hline Regression & 1 & 16153.80 & 16153.80 & 17.4596 & 0.001082 \\
Residual & 13 & 12027.72 & 925.21 & & \\
Total & 14 & 28181.52 & & & \\
\hline
\end{tabular}


Furthermore, a simple linear regression suggested that 41 percent of this variation in quantity could be attributed to publication year. This data suggests that a statistically significant relationship does exist between the quantity of CEDs published by Indian subsidiaries and the year of publication.

Table B. 29: Regression statistics for the quantity of CEDs from Indian subsidiary corporations from 20022016

\begin{tabular}{|ll|}
\hline Regression Statistics & \\
\hline Multiple R & 0.6466 \\
R Square & 0.4181 \\
Adjusted R Square & 0.3733 \\
Standard Error & 15.8457 \\
Observations & 15 \\
\hline
\end{tabular}

\begin{tabular}{|llllll|}
\hline ANOVA & Df & SS & MS & F & Significance F \\
\hline Regression & 1 & 2344.95 & 2344.95 & 9.3392 & 0.0092 \\
Residual & 13 & 3264.11 & 251.08 & & \\
Total & 14 & 5609.06 & & & \\
\hline
\end{tabular}

Furthermore, a simple linear regression suggested that 62 percent of this variation in quantity could be attributed to publication year. This data suggests that a statistically significant relationship does exist between the quantity of CEDs published by parent multinational corporations and the year of publication. 
Table B. 30: Regression statistics for the quantity of CEDs from parent multinational corporations from 2002-2016

\begin{tabular}{|ll|}
\hline Regression Statistics & \\
\hline Multiple R & 0.7914 \\
R Square & 0.6263 \\
Adjusted R Square & 0.5976 \\
Standard Error & 86.0522 \\
Observations & 15 \\
\hline
\end{tabular}

\begin{tabular}{|llllll|}
\hline ANOVA & Df & SS & MS & F & Significance F \\
\hline Regression & 1 & 161352.01 & 161352.01 & 21.79 & 0.00044 \\
Residual & 13 & 96264.72 & 7404.98 & & \\
Total & 14 & 257616.73 & & & \\
\hline
\end{tabular}




\section{B.3 CED Quality}

\section{B.3.1 Quality analysis for Coromandel}

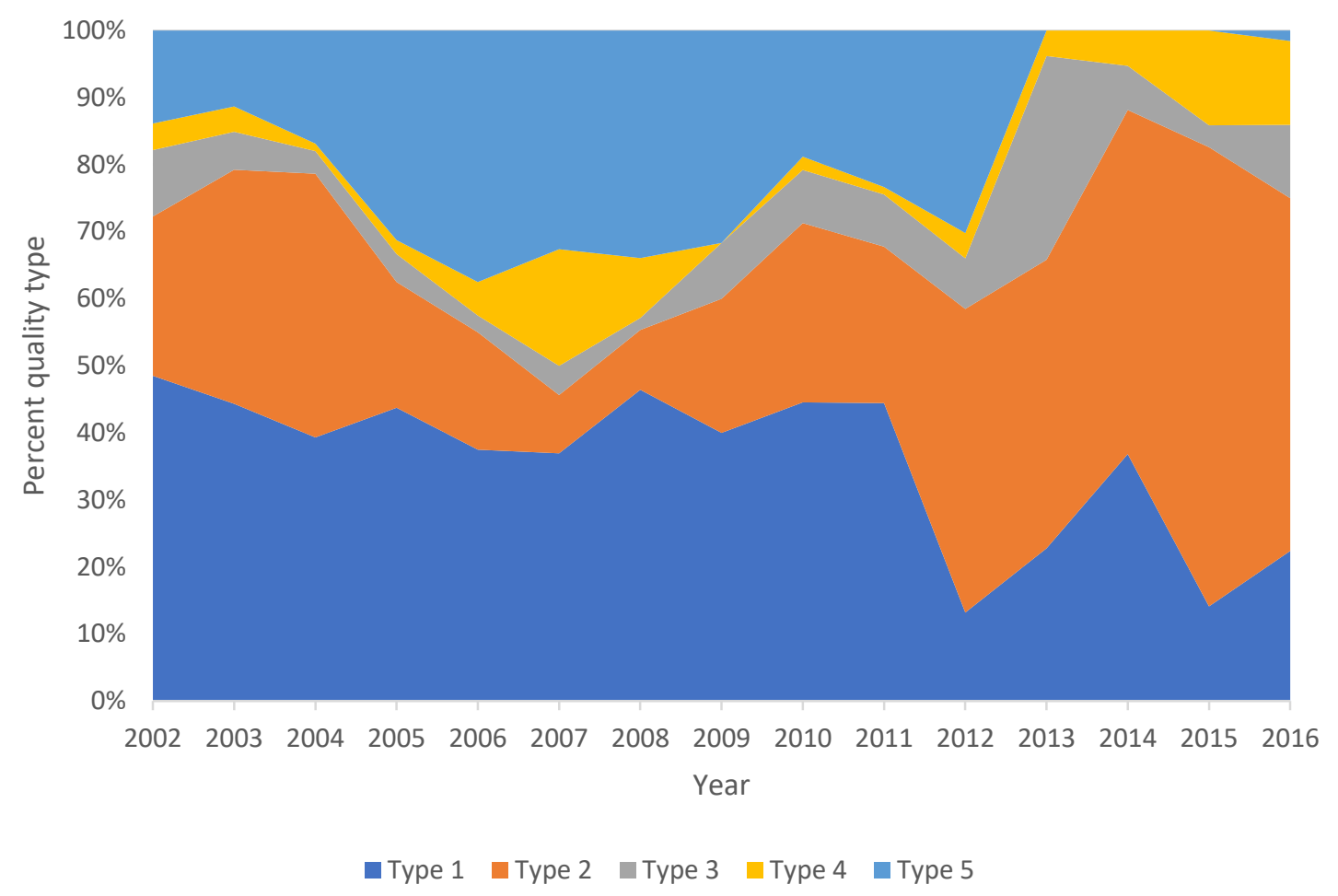

Figure B. 16: Quality composition of disclosures from Coromandel from 2002-2016. Type $1(p=0.003128)$, Type $2(p=0.011438)$, Type $3(p=0.229464)$, Type $4(p=0.236308)$, Type $5(p=0.067912)$.

Table B. 31:Regression statistics for the proportion of quality type 1 of CEDs from Coromandel from 2002-2016

\begin{tabular}{lr}
\hline \multicolumn{2}{c}{ Regression Statistics } \\
\hline Multiple R & 0.708234 \\
R Square & 0.501595 \\
Adjusted R & \\
Square & 0.463256 \\
Standard Error & 8.593829 \\
Observations & 15 \\
\hline
\end{tabular}

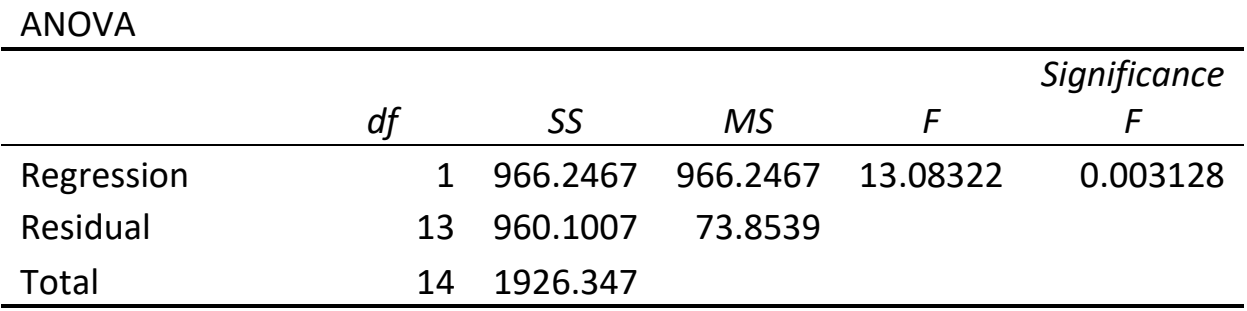


Table B. 32: Regression statistics for the proportion of quality type 2 of CEDs from Coromandel from 2002-2016

\begin{tabular}{|c|c|c|c|c|c|}
\hline \multicolumn{2}{|c|}{ Regression Statistics } & & & & \\
\hline Multiple R & 0.632259 & & & & \\
\hline $\begin{array}{l}\text { R Square } \\
\text { Adjusted R }\end{array}$ & 0.399752 & & & & \\
\hline Square & 0.353579 & & & & \\
\hline Standard Error & 14.03165 & & & & \\
\hline Observations & 15 & & & & \\
\hline \multicolumn{6}{|l|}{ ANOVA } \\
\hline & $d f$ & SS & $M S$ & $F$ & $\begin{array}{c}\text { Significance } \\
F \\
\end{array}$ \\
\hline Regression & 1 & 1704.59 & 1704.59 & 8.657703 & 0.011438 \\
\hline Residual & 13 & 2559.532 & 196.8871 & & \\
\hline Total & 14 & 4264.122 & & & \\
\hline
\end{tabular}

Table B. 33: : Regression statistics for the proportion of quality type 3 of CEDs from Coromandel from 2002-2016

\begin{tabular}{|c|c|c|c|c|c|}
\hline \multicolumn{2}{|c|}{ Regression Statistics } & & & & \\
\hline Multiple R & 0.330134 & & & & \\
\hline $\begin{array}{l}\text { R Square } \\
\text { Adjusted R }\end{array}$ & 0.108989 & & & & \\
\hline Square & 0.040449 & & & & \\
\hline Standard Error & 6.724291 & & & & \\
\hline Observations & 15 & & & & \\
\hline \multicolumn{6}{|l|}{ ANOVA } \\
\hline & $d f$ & SS & $M S$ & $F$ & $\begin{array}{c}\text { Significance } \\
F\end{array}$ \\
\hline Regression & 1 & 71.90087 & 71.90087 & 1.590161 & 0.229464 \\
\hline Residual & 13 & 587.8092 & 45.21609 & & \\
\hline Total & 14 & 659.7101 & & & \\
\hline
\end{tabular}


Table B. 34: Regression statistics for the proportion of quality type 4 of CEDs from Coromandel from 2002-2016

\begin{tabular}{|c|c|c|c|c|c|}
\hline \multicolumn{2}{|c|}{ Regression Statistics } & & & & \\
\hline Multiple R & 0.325602 & & & & \\
\hline $\begin{array}{l}\text { R Square } \\
\text { Adjusted R }\end{array}$ & 0.106016 & & & & \\
\hline Square & 0.037248 & & & & \\
\hline Standard Error & 5.11562 & & & & \\
\hline Observations & 15 & & & & \\
\hline \multicolumn{6}{|l|}{ ANOVA } \\
\hline & $d f$ & SS & MS & $F$ & $\begin{array}{c}\text { Significance } \\
F\end{array}$ \\
\hline Regression & 1 & 40.3444 & 40.3444 & 1.541653 & 0.236308 \\
\hline Residual & 13 & 340.2044 & 26.16957 & & \\
\hline Total & 14 & 380.5488 & & & \\
\hline
\end{tabular}

Table B. 35: Regression statistics for the proportion of quality type 5 of CEDs from Coromandel from 2002-2016

\begin{tabular}{|c|c|c|c|c|c|}
\hline \multicolumn{2}{|c|}{ Regression Statistics } & & & & \\
\hline Multiple R & 0.48342 & & & & \\
\hline R Square & 0.233695 & & & & \\
\hline Adjusted R & & & & & \\
\hline Square & 0.174748 & & & & \\
\hline Standard Error & 12.57255 & & & & \\
\hline Observations & 15 & & & & \\
\hline \multicolumn{6}{|l|}{ ANOVA } \\
\hline & & & & & Significance \\
\hline & $d f$ & SS & MS & $F$ & $F$ \\
\hline Regression & 1 & 626.6677 & 626.6677 & 3.964518 & 0.067912 \\
\hline Residual & 13 & 2054.898 & 158.0691 & & \\
\hline Total & 14 & 2681.566 & & & \\
\hline
\end{tabular}




\section{B.3.2 Quality analysis for Rallis India}

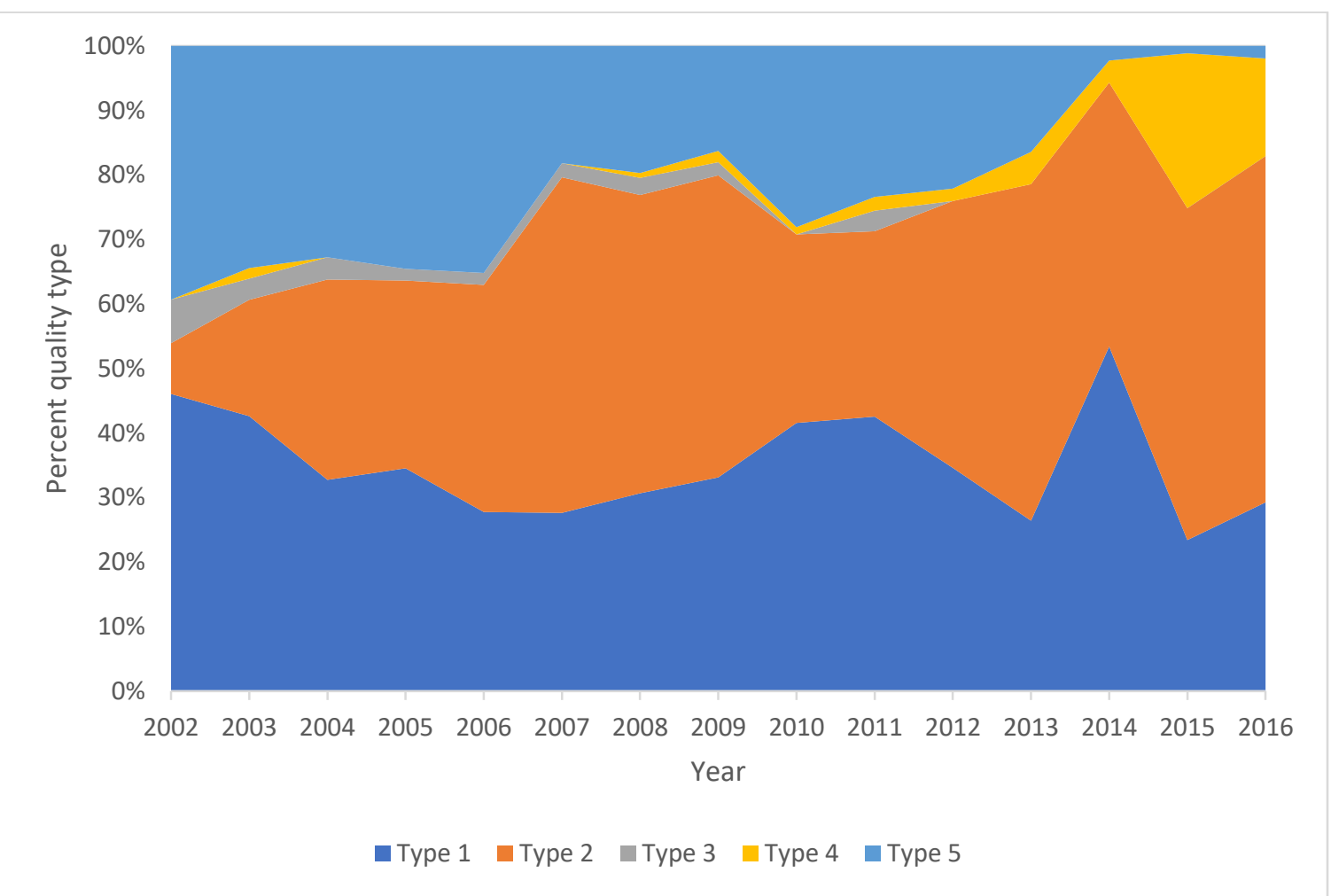

Figure B. 17: Quality composition of disclosures from Rallis India Ltd from 2002-2016. Type 1 $(p=0.495358)$, Type $2(p=0.00222)$, Type $3(p=0.00024)$, Type $4(p=0.005455)$, Type $5(p=8.65 E-06)$.

Table B. 36: Regression statistics for the proportion of quality type 1 of CEDs from Rallis India Ltd from 2002-2016

\begin{tabular}{|c|c|c|c|c|c|}
\hline \multicolumn{2}{|c|}{ Regression Statistics } & & & & \\
\hline Multiple R & 0.19098 & & & & \\
\hline $\begin{array}{l}\text { R Square } \\
\text { Adjusted R }\end{array}$ & 0.036473 & & & & \\
\hline Square & -0.03764 & & & & \\
\hline Standard Error & 8.581925 & & & & \\
\hline Observations & 15 & & & & \\
\hline \multicolumn{6}{|l|}{ ANOVA } \\
\hline & $d f$ & SS & MS & $F$ & $\begin{array}{c}\text { Significance } \\
F \\
\end{array}$ \\
\hline Regression & 1 & 36.24311 & 36.24311 & 0.492103 & 0.495358 \\
\hline Residual & 13 & 957.4426 & 73.64943 & & \\
\hline Total & 14 & 993.6858 & & & \\
\hline
\end{tabular}


Table B. 37: Regression statistics for the proportion of quality type 2 of CEDs from Rallis India Ltd from 2002-2016

\begin{tabular}{|c|c|c|c|c|c|}
\hline \multicolumn{2}{|c|}{ Regression Statistics } & & & & \\
\hline Multiple R & 0.725146 & & & & \\
\hline R Square & 0.525836 & & & & \\
\hline Adjusted R & & & & & \\
\hline Square & 0.489362 & & & & \\
\hline Standard Error & 9.718139 & & & & \\
\hline Observations & 15 & & & & \\
\hline \multicolumn{6}{|l|}{ ANOVA } \\
\hline & $d f$ & SS & $M S$ & $F$ & $\begin{array}{c}\text { Significance } \\
F\end{array}$ \\
\hline Regression & 1 & 1361.544 & 1361.544 & 14.41669 & 0.00222 \\
\hline Residual & 13 & 1227.749 & 94.44222 & & \\
\hline Total & 14 & 2589.293 & & & \\
\hline
\end{tabular}

Table B. 38: Regression statistics for the proportion of quality type 3 of CEDs from Rallis India Ltd from 2002-2016

\begin{tabular}{|c|c|c|c|c|c|}
\hline \multicolumn{2}{|c|}{ Regression Statistics } & & & & \\
\hline Multiple R & 0.811492 & & & & \\
\hline $\begin{array}{l}\text { R Square } \\
\text { Adjusted R }\end{array}$ & 0.658519 & & & & \\
\hline Square & 0.632252 & & & & \\
\hline Standard Error & 1.164507 & & & & \\
\hline Observations & 15 & & & & \\
\hline \multicolumn{6}{|l|}{ ANOVA } \\
\hline & & & & & Significance \\
\hline & $d f$ & SS & $M S$ & $F$ & $F$ \\
\hline Regression & 1 & 33.99616 & 33.99616 & 25.0695 & 0.00024 \\
\hline Residual & 13 & 17.62899 & 1.356076 & & \\
\hline Total & 14 & 51.62515 & & & \\
\hline
\end{tabular}


Table B. 39: Regression statistics for the proportion of quality type 4 of CEDs from Rallis India Ltd from 2002-2016

\begin{tabular}{|c|c|c|c|c|c|}
\hline \multicolumn{2}{|c|}{ Regression Statistics } & & & & \\
\hline Multiple R & 0.678171 & & & & \\
\hline $\begin{array}{l}\text { R Square } \\
\text { Adjusted R }\end{array}$ & 0.459916 & & & & \\
\hline Square & 0.418371 & & & & \\
\hline Standard Error & 5.163079 & & & & \\
\hline Observations & 15 & & & & \\
\hline \multicolumn{6}{|l|}{ ANOVA } \\
\hline & $d f$ & SS & MS & $F$ & $\begin{array}{c}\text { Significance } \\
F \\
\end{array}$ \\
\hline Regression & 1 & 295.1057 & 295.1057 & 11.07031 & 0.005455 \\
\hline Residual & 13 & 346.546 & 26.65739 & & \\
\hline Total & 14 & 641.6517 & & & \\
\hline
\end{tabular}

Table B. 40: Regression statistics for the proportion of quality type 5 of CEDs from Rallis India Ltd from 2002-2016

\begin{tabular}{|c|c|c|c|c|c|}
\hline \multicolumn{2}{|c|}{ Regression Statistics } & & & & \\
\hline Multiple R & 0.890365 & & & & \\
\hline R Square & 0.792751 & & & & \\
\hline Adjusted R & & & & & \\
\hline Square & 0.776808 & & & & \\
\hline Standard Error & 5.988195 & & & & \\
\hline Observations & 15 & & & & \\
\hline \multicolumn{6}{|l|}{ ANOVA } \\
\hline & & & & & Significance \\
\hline & $d f$ & SS & $M S$ & $F$ & $F$ \\
\hline Regression & 1 & 1783.111 & 1783.111 & 49.72635 & $8.65 \mathrm{E}-06$ \\
\hline Residual & 13 & 466.1602 & 35.85847 & & \\
\hline Total & 14 & 2249.271 & & & \\
\hline
\end{tabular}

\section{B.3.3 Quality analysis of UPL Limited}




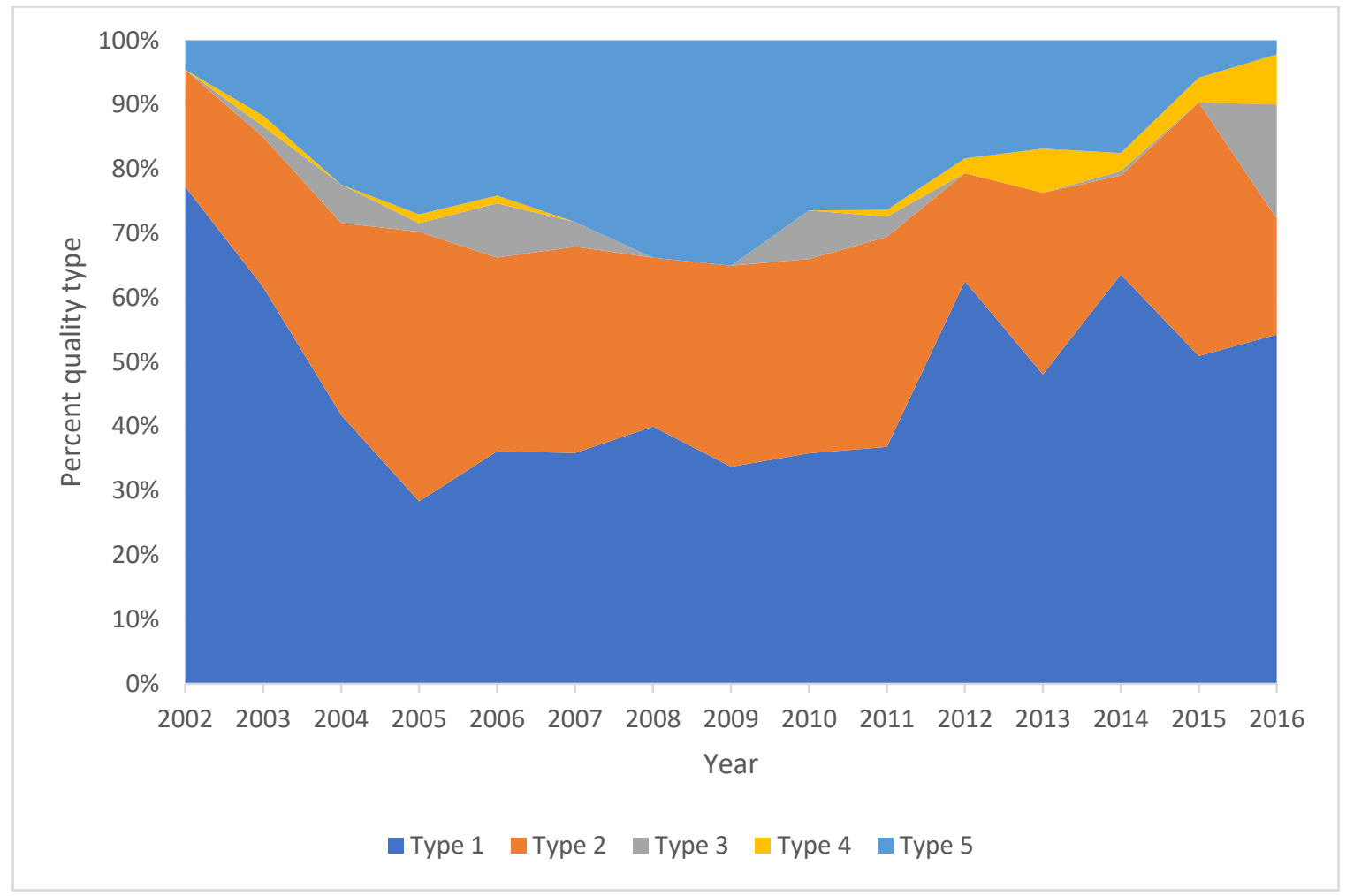

Figure B. 18: Quality composition of disclosure from UPL from 2002-2016. Type 1 ( $p=0.872292)$, Type 2 $(p=0.635764)$, Type $3(p=0.462509)$, Type $4(p=0.003868)$, Type $5(p=0.416638)$.

Table B. 41: Regression statistics for the proportion of quality type 1 of CEDs from UPL from 2002-2016

\begin{tabular}{lr}
\hline \multicolumn{2}{c}{ Regression Statistics } \\
\hline Multiple R & 0.045425 \\
R Square & 0.002063 \\
Adjusted R & \\
Square & -0.0747 \\
Standard Error & 14.63071 \\
Observations & 15 \\
\hline
\end{tabular}

\begin{tabular}{|c|c|c|c|c|c|}
\hline & $d f$ & SS & $M S$ & $F$ & $\begin{array}{c}\text { Significance } \\
F\end{array}$ \\
\hline Regression & 1 & 5.753805 & 5.753805 & 0.02688 & 0.872292 \\
\hline Residual & 13 & 2782.75 & 214.0577 & & \\
\hline Total & 14 & 2788.504 & & & \\
\hline
\end{tabular}

Table B. 42: : Regression statistics for the proportion of quality type 2 of CEDs from UPL from 2002-2016 


\begin{tabular}{|c|c|c|c|c|c|}
\hline \multicolumn{2}{|c|}{ Regression Statistics } & & & & \\
\hline Multiple R & 0.133307 & & & & \\
\hline $\begin{array}{l}\text { R Square } \\
\text { Adjusted R }\end{array}$ & 0.017771 & & & & \\
\hline Square & -0.05779 & & & & \\
\hline Standard Error & 8.189658 & & & & \\
\hline Observations & 15 & & & & \\
\hline \multicolumn{6}{|l|}{ ANOVA } \\
\hline & & & & & Significance \\
\hline & $d f$ & SS & $M S$ & $F$ & $F$ \\
\hline Regression & 1 & 15.77505 & 15.77505 & 0.235201 & 0.635764 \\
\hline Residual & 13 & 871.9165 & 67.0705 & & \\
\hline Total & 14 & 887.6916 & & & \\
\hline
\end{tabular}

Table B. 43: Regression statistics for the proportion of quality type 3 of CEDs from UPL from 2002-2016

\begin{tabular}{|c|c|c|c|c|c|}
\hline \multicolumn{2}{|c|}{ Regression Statistics } & & & & \\
\hline Multiple R & 0.205493 & & & & \\
\hline R Square & 0.042227 & & & & \\
\hline Adjusted R & & & & & \\
\hline Square & -0.03145 & & & & \\
\hline Standard Error & 4.997018 & & & & \\
\hline Observations & 15 & & & & \\
\hline \multicolumn{6}{|l|}{ ANOVA } \\
\hline & & & & & Significance \\
\hline & $d f$ & SS & MS & $F$ & $F$ \\
\hline Regression & 1 & 14.31183 & 14.31183 & 0.573157 & 0.462509 \\
\hline Residual & 13 & 324.6124 & 24.97019 & & \\
\hline Total & 14 & 338.9243 & & & \\
\hline
\end{tabular}

Table B. 44: Regression statistics for the proportion of quality type 4 of CEDs from UPL from 2002-2016 Regression Statistics 


\begin{tabular}{|c|c|c|c|c|c|}
\hline Multiple R & 0.697159 & & & & \\
\hline R Square & 0.486031 & & & & \\
\hline Adjusted R & & & & & \\
\hline Square & 0.446495 & & & & \\
\hline Standard Error & 1.859039 & & & & \\
\hline Observations & 15 & & & & \\
\hline \multicolumn{6}{|l|}{ ANOVA } \\
\hline & & & & & Significance \\
\hline & $d f$ & SS & $M S$ & $F$ & $F$ \\
\hline Regression & 1 & 42.48617 & 42.48617 & 12.29337 & 0.003868 \\
\hline Residual & 13 & 44.92832 & 3.456025 & & \\
\hline Total & 14 & 87.41449 & & & \\
\hline
\end{tabular}

Table B. 45: Regression statistics for the proportion of quality type 5 of CEDs from UPL from 2002-2016

\begin{tabular}{|c|c|c|c|c|c|}
\hline \multicolumn{2}{|c|}{ Regression Statistics } & & & & \\
\hline Multiple R & 0.226638 & & & & \\
\hline R Square & 0.051365 & & & & \\
\hline Adjusted R & & & & & \\
\hline Square & -0.02161 & & & & \\
\hline Standard Error & 10.40323 & & & & \\
\hline Observations & 15 & & & & \\
\hline \multicolumn{6}{|l|}{ ANOVA } \\
\hline & & & & & Significance \\
\hline & $d f$ & SS & $M S$ & $F$ & $F$ \\
\hline Regression & 1 & 76.18092 & 76.18092 & 0.703898 & 0.416638 \\
\hline Residual & 13 & 1406.953 & 108.2272 & & \\
\hline Total & 14 & 1483.134 & & & \\
\hline
\end{tabular}

\section{B.3.4 Quality analysis of Zuari}




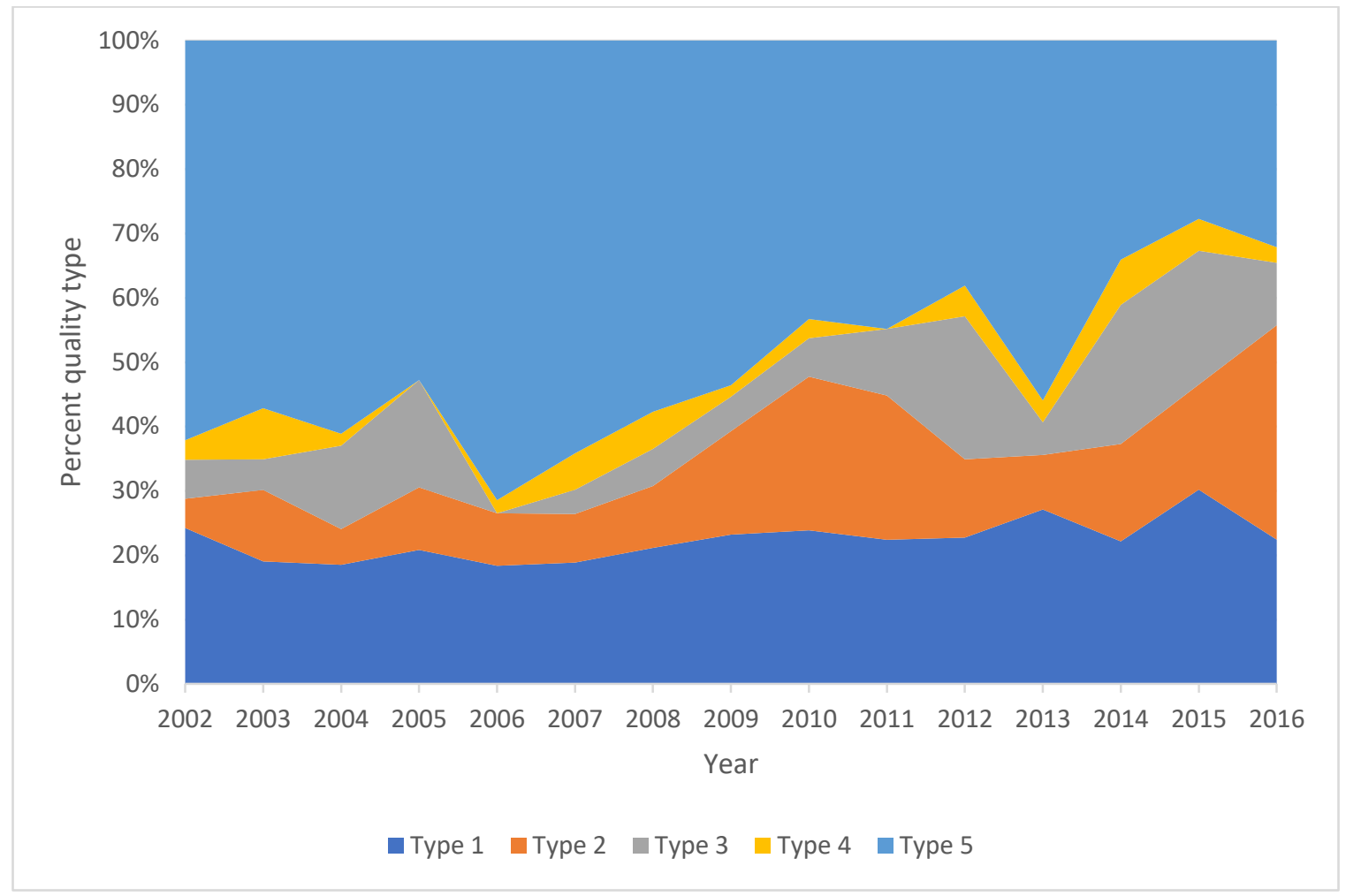

Figure B. 19: Quality composition of disclosure from Zuari from 2002-2016. Type 1 ( $p=0.02035)$, Type 2 $(p=0.005926)$, Type $3(p=0.097645)$, Type $4(p=0.787839)$, Type $5(p=0.000238)$.

Table B. 46: Regression statistics for the proportion of quality type 1 of CEDs from Zuari from 2002-2016

\begin{tabular}{lr}
\hline \multicolumn{2}{c}{ Regression Statistics } \\
\hline Multiple R & 0.590944 \\
R Square & 0.349214 \\
Adjusted R & \\
Square & 0.299154 \\
Standard Error & 2.726637 \\
Observations & 15 \\
\hline
\end{tabular}

\begin{tabular}{lrrrrc} 
ANOVA & & & & & \multicolumn{2}{c}{ Significance } \\
& $d f$ & \multicolumn{1}{c}{ SS } & \multicolumn{1}{c}{ MS } & \multicolumn{1}{c}{$F$} & \multicolumn{1}{c}{$F$} \\
\hline Regression & 1 & 51.86235 & 51.86235 & 6.975856 & 0.02035 \\
Residual & 13 & 96.64914 & 7.43455 & & \\
Total & 14 & 148.5115 & & & \\
\hline
\end{tabular}

Table B. 47: Regression statistics for the proportion of quality type 2 of CEDs from Zuari from 2002-2016 Regression Statistics 


\begin{tabular}{|c|c|c|c|c|c|}
\hline Multiple R & 0.673388 & & & & \\
\hline $\begin{array}{l}\text { R Square } \\
\text { Adjusted R }\end{array}$ & 0.453451 & & & & \\
\hline Square & 0.411409 & & & & \\
\hline Standard Error & 6.037609 & & & & \\
\hline Observations & 15 & & & & \\
\hline \multicolumn{6}{|l|}{ ANOVA } \\
\hline & $d f$ & SS & $M S$ & $F$ & $\begin{array}{c}\text { Significance } \\
F\end{array}$ \\
\hline Regression & 1 & 393.1645 & 393.1645 & 10.7856 & 0.005926 \\
\hline Residual & 13 & 473.8854 & 36.45272 & & \\
\hline Total & 14 & 867.0499 & & & \\
\hline
\end{tabular}

Table B. 48: Regression statistics for the proportion of quality type 3 of CEDs from Zuari from 2002-2016

\begin{tabular}{|c|c|c|c|c|c|}
\hline \multicolumn{2}{|c|}{ Regression Statistics } & & & & \\
\hline Multiple R & 0.443623 & & & & \\
\hline R Square & 0.196801 & & & & \\
\hline Adjusted R & & & & & \\
\hline Square & 0.135017 & & & & \\
\hline Standard Error & 6.650444 & & & & \\
\hline Observations & 15 & & & & \\
\hline \multicolumn{6}{|l|}{ ANOVA } \\
\hline & $d f$ & SS & $M S$ & $F$ & $\begin{array}{c}\text { Significance } \\
F\end{array}$ \\
\hline Regression & 1 & 140.8801 & 140.8801 & 3.185287 & 0.097645 \\
\hline Residual & 13 & 574.9692 & 44.2284 & & \\
\hline Total & 14 & 715.8493 & & & \\
\hline
\end{tabular}

Table B. 49: Regression statistics for the proportion of quality type 4 of CEDs from Zuari from 2002-2016 


\begin{tabular}{|c|c|c|c|c|c|}
\hline Multiple R & 0.075977 & & & & \\
\hline R Square & 0.005772 & & & & \\
\hline Adjusted R & & & & & \\
\hline Square & -0.07071 & & & & \\
\hline Standard Error & 2.465665 & & & & \\
\hline Observations & 15 & & & & \\
\hline \multicolumn{6}{|l|}{ ANOVA } \\
\hline & & & & & Significance \\
\hline & $d f$ & SS & $M S$ & $F$ & $F$ \\
\hline Regression & 1 & 0.458869 & 0.458869 & 0.075478 & 0.787839 \\
\hline Residual & 13 & 79.03353 & 6.079502 & & \\
\hline Total & 14 & 79.4924 & & & \\
\hline
\end{tabular}

Table B. 50 : Regression statistics for the proportion of quality type 5 of CEDs from Zuari from 2002-2016

\begin{tabular}{|c|c|c|c|c|c|}
\hline \multicolumn{2}{|c|}{ Regression Statistics } & & & & \\
\hline Multiple R & 0.811749 & & & & \\
\hline R Square & 0.658936 & & & & \\
\hline Adjusted R & & & & & \\
\hline Square & 0.6327 & & & & \\
\hline Standard Error & 7.897025 & & & & \\
\hline Observations & 15 & & & & \\
\hline \multicolumn{6}{|l|}{ ANOVA } \\
\hline & & & & & Significance \\
\hline & $d f$ & SS & MS & $F$ & $F$ \\
\hline Regression & 1 & 1566.309 & 1566.309 & 25.116 & 0.000238 \\
\hline Residual & 13 & 810.719 & 62.363 & & \\
\hline Total & 14 & 2377.028 & & & \\
\hline
\end{tabular}

\section{B.3.5 Quality analysis of I-BASF}




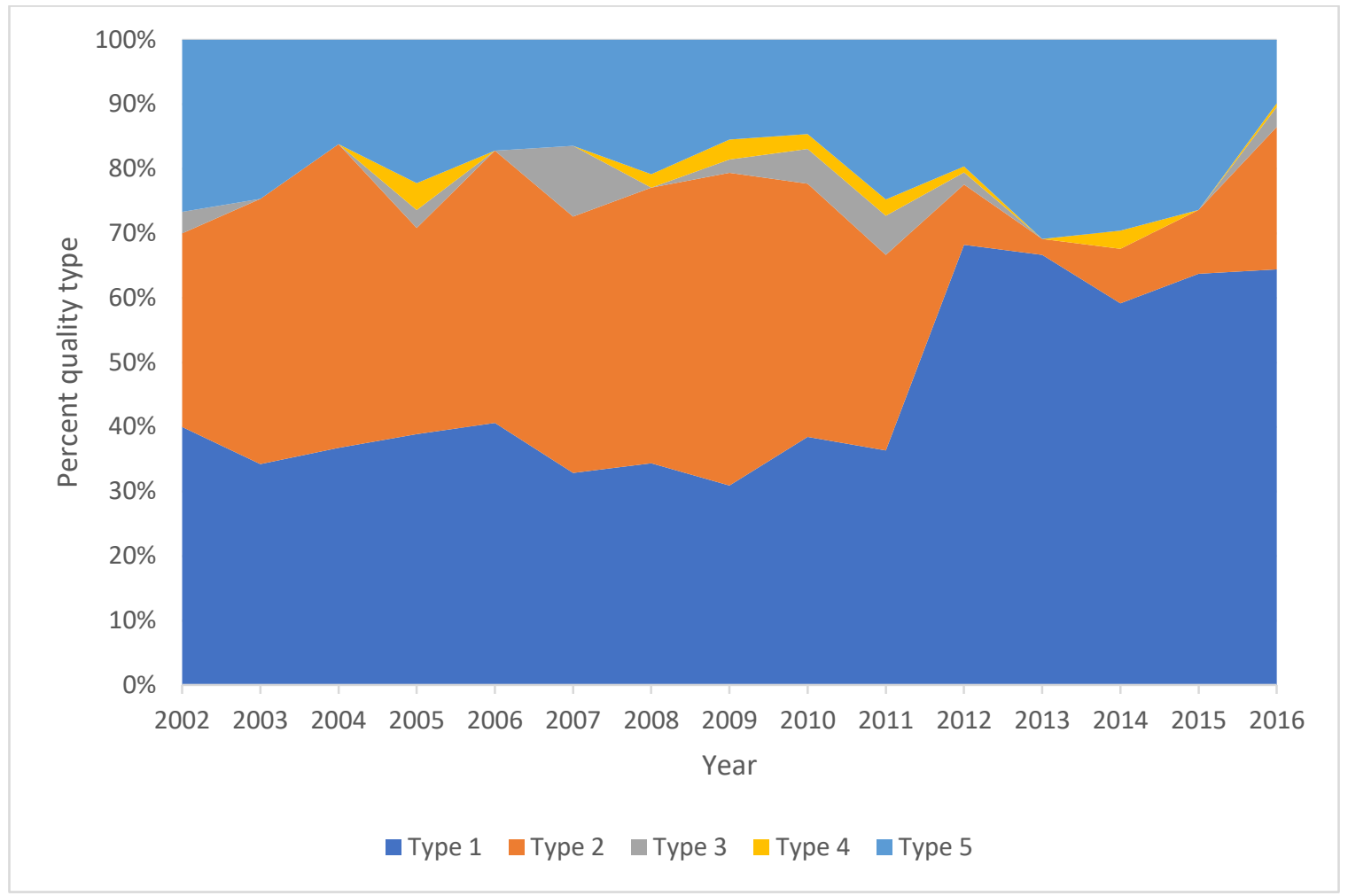

Figure B. 20: Quality composition of disclosure from I-BASF from 2002-2016. Type 1 ( $p=0.001141)$, Type 2 ( $p=0.004024)$, Type $3(p=0.83187)$, Type $4(p=0.701068)$, Type $5(p=0.913945)$.

Table B. 51: Regression statistics for the proportion of quality type 1 of CEDs from I-BASF from 2002-2016

\begin{tabular}{lr}
\hline \multicolumn{2}{c}{ Regression Statistics } \\
\hline Multiple R & 0.754881 \\
R Square & 0.569845 \\
Adjusted R & \\
Square & 0.536756 \\
Standard Error & 9.573284 \\
Observations & 15 \\
\hline
\end{tabular}

\begin{tabular}{lrcccc} 
ANOVA & & & & & \\
\hline & $d f$ & SS & MS & \multicolumn{1}{c}{$F$} & \multicolumn{1}{c}{ Significance } \\
\hline Regression & 1 & 1578.325 & 1578.325 & 17.22164 & 0.001141 \\
Residual & 13 & 1191.421 & 91.64776 & & \\
Total & 14 & 2769.746 & & & \\
\hline
\end{tabular}

Table B. 52: Regression statistics for the proportion of quality type 2 of CEDs from I-BASF from 2002-2016 Regression Statistics 


\begin{tabular}{|c|c|c|c|c|c|}
\hline Multiple R & 0.695048 & & & & \\
\hline $\begin{array}{l}\text { R Square } \\
\text { Adjusted R }\end{array}$ & 0.483091 & & & & \\
\hline Square & 0.443329 & & & & \\
\hline Standard Error & 11.56311 & & & & \\
\hline Observations & 15 & & & & \\
\hline \multicolumn{6}{|l|}{ ANOVA } \\
\hline & & & & & Significance \\
\hline & $d f$ & SS & $M S$ & $F$ & $F$ \\
\hline Regression & 1 & 1624.456 & 1624.456 & 12.14951 & 0.004024 \\
\hline Residual & 13 & 1738.172 & 133.7055 & & \\
\hline Total & 14 & 3362.628 & & & \\
\hline
\end{tabular}

Table B. 53: Regression statistics for the proportion of quality type 3 of CEDs from I-BASF from 2002-2016

\begin{tabular}{|c|c|c|c|c|c|}
\hline \multicolumn{2}{|c|}{ Regression Statistics } & & & & \\
\hline Multiple R & 0.05997 & & & & \\
\hline R Square & 0.003596 & & & & \\
\hline Adjusted R & & & & & \\
\hline Square & -0.07305 & & & & \\
\hline Standard Error & 3.248991 & & & & \\
\hline Observations & 15 & & & & \\
\hline \multicolumn{6}{|l|}{ ANOVA } \\
\hline & $d f$ & SS & $M S$ & $F$ & $\begin{array}{c}\text { Significance } \\
F\end{array}$ \\
\hline Regression & 1 & 0.495312 & 0.495312 & 0.046923 & 0.83187 \\
\hline Residual & 13 & 137.2272 & 10.55594 & & \\
\hline Total & 14 & 137.7225 & & & \\
\hline
\end{tabular}

Table B. 54: Regression statistics for the proportion of quality type 4 of CEDs from I-BASF from 2002-2016 Regression Statistics 


\begin{tabular}{|c|c|c|c|c|c|}
\hline Multiple R & 0.108213 & & & & \\
\hline R Square & 0.01171 & & & & \\
\hline Adjusted R & & & & & \\
\hline Square & -0.06431 & & & & \\
\hline Standard Error & 1.49085 & & & & \\
\hline Observations & 15 & & & & \\
\hline \multicolumn{6}{|l|}{ ANOVA } \\
\hline & & & & & Significance \\
\hline & $d f$ & SS & MS & $F$ & $F$ \\
\hline Regression & 1 & 0.342365 & 0.342365 & 0.154036 & 0.701068 \\
\hline Residual & 13 & 28.89424 & 2.222634 & & \\
\hline Total & 14 & 29.23661 & & & \\
\hline
\end{tabular}

Table B. 55: Regression statistics for the proportion of quality type 5 of CEDs from I-BASF from 2002-2016

\begin{tabular}{|c|c|c|c|c|c|}
\hline \multicolumn{2}{|c|}{ Regression Statistics } & & & & \\
\hline Multiple R & 0.030546 & & & & \\
\hline R Square & 0.000933 & & & & \\
\hline Adjusted R & & & & & \\
\hline Square & -0.07592 & & & & \\
\hline Standard Error & 6.308171 & & & & \\
\hline Observations & 15 & & & & \\
\hline \multicolumn{6}{|l|}{ ANOVA } \\
\hline & $d f$ & SS & $M S$ & $F$ & $\begin{array}{c}\text { Significance } \\
F\end{array}$ \\
\hline Regression & 1 & 0.483119 & 0.483119 & 0.012141 & 0.913945 \\
\hline Residual & 13 & 517.3092 & 39.79302 & & \\
\hline Total & 14 & 517.7923 & & & \\
\hline
\end{tabular}

\section{B.3.6 Quality analysis of I-Bayer}




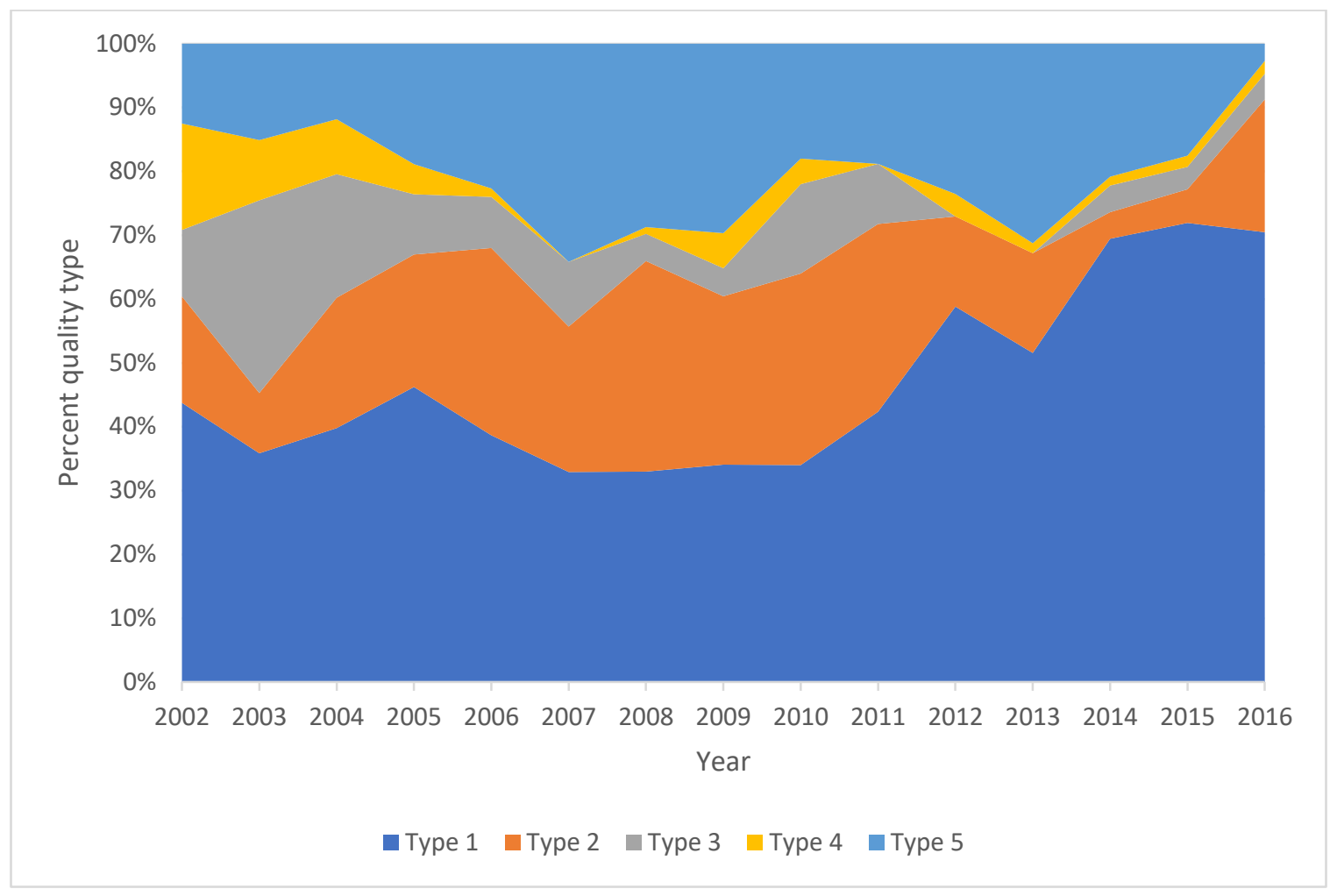

Figure B. 21: Quality composition of disclosure from I-Bayer from 2002-2016. Type 1 ( $p=0.001916)$, Type $2(p=0.396966)$, Type $(p=0.005518)$, Type $4(p=0.006641)$, Type $5(p=0.990807)$.

Table B. 56: Regression statistics for the proportion of quality type 1 of CEDs from I-Bayer from 20022016

\begin{tabular}{lr}
\hline \multicolumn{2}{c}{ Regression Statistics } \\
\hline Multiple R & 0.732064 \\
R Square & 0.535917 \\
Adjusted R & \\
Square & 0.500219 \\
Standard Error & 10.08023 \\
Observations & 15 \\
\hline
\end{tabular}

ANOVA

\begin{tabular}{|c|c|c|c|c|c|}
\hline & $d f$ & SS & $M S$ & $F$ & $\begin{array}{c}\text { Significance } \\
F\end{array}$ \\
\hline Regression & 1 & 1525.409 & 1525.409 & 15.01225 & 0.001916 \\
\hline Residual & 13 & 1320.942 & 101.6109 & & \\
\hline Total & 14 & 2846.351 & & & \\
\hline
\end{tabular}

Table B. 57: Regression statistics for the proportion of quality type 2 of CEDs from I-Bayer from 20022016 


\begin{tabular}{|c|c|c|c|c|c|}
\hline \multicolumn{2}{|c|}{ Regression Statistics } & & & & \\
\hline Multiple R & 0.236072 & & & & \\
\hline $\begin{array}{l}\text { R Square } \\
\text { Adjusted R }\end{array}$ & 0.05573 & & & & \\
\hline Square & -0.01691 & & & & \\
\hline Standard Error & 9.091974 & & & & \\
\hline Observations & 15 & & & & \\
\hline \multicolumn{6}{|l|}{ ANOVA } \\
\hline & & & & & Significance \\
\hline & $d f$ & SS & $M S$ & $F$ & $F$ \\
\hline Regression & 1 & 63.42401 & 63.42401 & 0.767251 & 0.396966 \\
\hline Residual & 13 & 1074.632 & 82.66399 & & \\
\hline Total & 14 & 1138.056 & & & \\
\hline
\end{tabular}

Table B. 58: Regression statistics for the proportion of quality type 3 of CEDs from I-Bayer from 20022016

\begin{tabular}{|c|c|c|c|c|c|}
\hline \multicolumn{2}{|c|}{ Regression Statistics } & & & & \\
\hline Multiple R & 0.677508 & & & & \\
\hline $\begin{array}{l}\text { R Square } \\
\text { Adjusted R }\end{array}$ & 0.459017 & & & & \\
\hline Square & 0.417403 & & & & \\
\hline Standard Error & 6.013043 & & & & \\
\hline Observations & 15 & & & & \\
\hline \multicolumn{6}{|l|}{ ANOVA } \\
\hline & $d f$ & SS & MS & $F$ & $\begin{array}{c}\text { Significance } \\
F\end{array}$ \\
\hline Regression & 1 & 398.8196 & 398.8196 & 11.03032 & 0.005518 \\
\hline Residual & 13 & 470.0369 & 36.15668 & & \\
\hline Total & 14 & 868.8565 & & & \\
\hline
\end{tabular}


Table B. 59: Regression statistics for the proportion of quality type 4 of CEDs from I-Bayer from 20022016

\begin{tabular}{|c|c|c|c|c|c|}
\hline \multicolumn{2}{|c|}{ Regression Statistics } & & & & \\
\hline Multiple R & 0.666667 & & & & \\
\hline $\begin{array}{l}\text { R Square } \\
\text { Adjusted R }\end{array}$ & 0.444446 & & & & \\
\hline Square & 0.401711 & & & & \\
\hline Standard Error & 3.480343 & & & & \\
\hline Observations & 15 & & & & \\
\hline \multicolumn{6}{|l|}{ ANOVA } \\
\hline & $d f$ & SS & MS & $F$ & $\begin{array}{c}\text { Significance } \\
F \\
\end{array}$ \\
\hline Regression & 1 & 125.9735 & 125.9735 & 10.40005 & 0.006641 \\
\hline Residual & 13 & 157.4662 & 12.11279 & & \\
\hline Total & 14 & 283.4398 & & & \\
\hline
\end{tabular}

Table B. 60: Regression statistics for the proportion of quality type 5 of CEDs from I-Bayer from 20022016

\begin{tabular}{|c|c|c|c|c|c|}
\hline \multicolumn{2}{|c|}{ Regression Statistics } & & & & \\
\hline Multiple R & 0.003258 & & & & \\
\hline R Square & $1.06 \mathrm{E}-05$ & & & & \\
\hline Adjusted R & & & & & \\
\hline Square & -0.07691 & & & & \\
\hline Standard Error & 8.657966 & & & & \\
\hline Observations & 15 & & & & \\
\hline \multicolumn{6}{|l|}{ ANOVA } \\
\hline & & & & & Significance \\
\hline & $d f$ & SS & $M S$ & $F$ & $F$ \\
\hline Regression & 1 & 0.010342 & 0.010342 & 0.000138 & 0.990807 \\
\hline Residual & 13 & 974.4849 & 74.96038 & & \\
\hline Total & 14 & 974.4952 & & & \\
\hline
\end{tabular}




\section{B.3.7 Quality analysis of I-Monsanto}

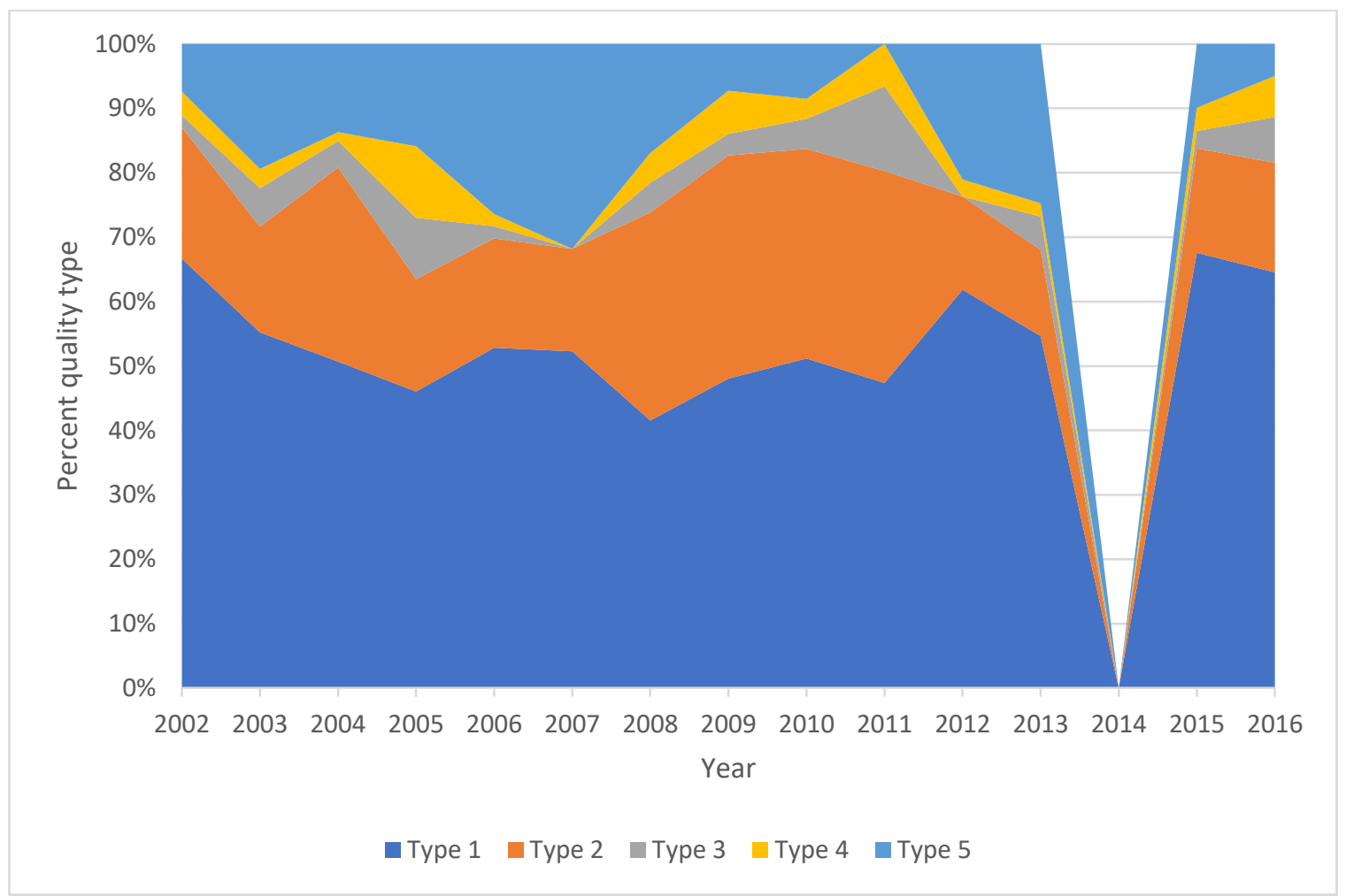

Figure B. 22: Quality composition of disclosure from I-Monsanto from 2002-2016. Type 1 ( $p=0.637622)$, Type 2 ( $p=0.329595)$, Type 3 ( $p=0.996855)$, Type $4(p=0.897474)$, Type $5(p=0.236979)$. 
Table B. 61: Regression statistics for the proportion of quality type 1 of CEDs from I-Monsanto from 2002-2016

\begin{tabular}{|c|c|c|c|c|c|}
\hline \multicolumn{2}{|c|}{ Regression Statistics } & & & & \\
\hline Multiple R & 0.132581 & & & & \\
\hline R Square & 0.017578 & & & & \\
\hline Adjusted R & & & & & \\
\hline Square & -0.05799 & & & & \\
\hline Standard Error & 16.47734 & & & & \\
\hline Observations & 15 & & & & \\
\hline \multicolumn{6}{|l|}{ ANOVA } \\
\hline & & & & & Significance \\
\hline & $d f$ & SS & $M S$ & $F$ & $F$ \\
\hline Regression & 1 & 63.15122 & 63.15122 & 0.232599 & 0.637622 \\
\hline Residual & 13 & 3529.537 & 271.5028 & & \\
\hline Total & 14 & 3592.688 & & & \\
\hline
\end{tabular}

Table B. 62: Regression statistics for the proportion of quality type 2 of CEDs from I-Monsanto from 2002-2016

\begin{tabular}{|c|c|c|c|c|c|}
\hline \multicolumn{2}{|c|}{ Regression Statistics } & & & & \\
\hline Multiple R & 0.270455 & & & & \\
\hline R Square & 0.073146 & & & & \\
\hline Adjusted R & & & & & \\
\hline Square & 0.00185 & & & & \\
\hline Standard Error & 9.727633 & & & & \\
\hline Observations & 15 & & & & \\
\hline \multicolumn{6}{|l|}{ ANOVA } \\
\hline & & & & & Significance \\
\hline & $d f$ & SS & MS & $F$ & $F$ \\
\hline Regression & 1 & 97.08179 & 97.08179 & 1.025943 & 0.329595 \\
\hline Residual & 13 & 1230.149 & 94.62684 & & \\
\hline Total & 14 & 1327.231 & & & \\
\hline
\end{tabular}

Table B. 63: Regression statistics for the proportion of quality type 3 of CEDs from I-Monsanto from 2002-2016 


\begin{tabular}{|c|c|c|c|c|c|}
\hline \multicolumn{2}{|c|}{ Regression Statistics } & & & & \\
\hline Multiple R & 0.001114 & & & & \\
\hline R Square & $1.24 \mathrm{E}-06$ & & & & \\
\hline Adjusted R & & & & & \\
\hline Square & -0.07692 & & & & \\
\hline Standard Error & 3.80334 & & & & \\
\hline Observations & 15 & & & & \\
\hline \multicolumn{6}{|l|}{ ANOVA } \\
\hline & & & & & Significance \\
\hline & $d f$ & SS & MS & $F$ & $F$ \\
\hline & & & & $1.61 \mathrm{E}-$ & \\
\hline Regression & 1 & 0.000233 & 0.000233 & 05 & 0.996855 \\
\hline Residual & 13 & 188.0502 & 14.4654 & & \\
\hline Total & 14 & 188.0504 & & & \\
\hline
\end{tabular}

Table B. 64: Regression statistics for the proportion of quality type 4 of CEDs from I-Monsanto from 2002-2016

\begin{tabular}{|c|c|c|c|c|c|}
\hline \multicolumn{2}{|c|}{ Regression Statistics } & & & & \\
\hline Multiple R & 0.036418 & & & & \\
\hline $\begin{array}{l}\text { R Square } \\
\text { Adjusted R }\end{array}$ & 0.001326 & & & & \\
\hline Square & -0.07549 & & & & \\
\hline Standard Error & 3.048483 & & & & \\
\hline Observations & 15 & & & & \\
\hline \multicolumn{6}{|l|}{ ANOVA } \\
\hline & $d f$ & SS & MS & $F$ & $\begin{array}{c}\text { Significance } \\
F \\
\end{array}$ \\
\hline Regression & 1 & 0.160446 & 0.160446 & 0.017265 & 0.897474 \\
\hline Residual & 13 & 120.8123 & 9.293251 & & \\
\hline Total & 14 & 120.9727 & & & \\
\hline
\end{tabular}

Table B. 65: Regression statistics for the proportion of quality type 5 of CEDs from I-Monsanto from 2002-2016

\begin{tabular}{lr}
\hline \multicolumn{2}{c}{ Regression Statistics } \\
\hline Multiple R & 0.325161
\end{tabular}




\begin{tabular}{|c|c|c|c|c|c|}
\hline R Square & 0.10573 & & & & \\
\hline Square & 0.03694 & & & & \\
\hline Standard Error & 9.409193 & & & & \\
\hline Observations & 15 & & & & \\
\hline \multicolumn{6}{|l|}{ ANOVA } \\
\hline & & & & & Significance \\
\hline & $d f$ & SS & MS & $F$ & $F$ \\
\hline Regression & 1 & 136.0744 & 136.0744 & 1.536993 & 0.236979 \\
\hline Residual & 13 & 1150.928 & 88.53291 & & \\
\hline Total & 14 & 1287.002 & & & \\
\hline
\end{tabular}

B.3.8 Quality analysis of I-Syngenta

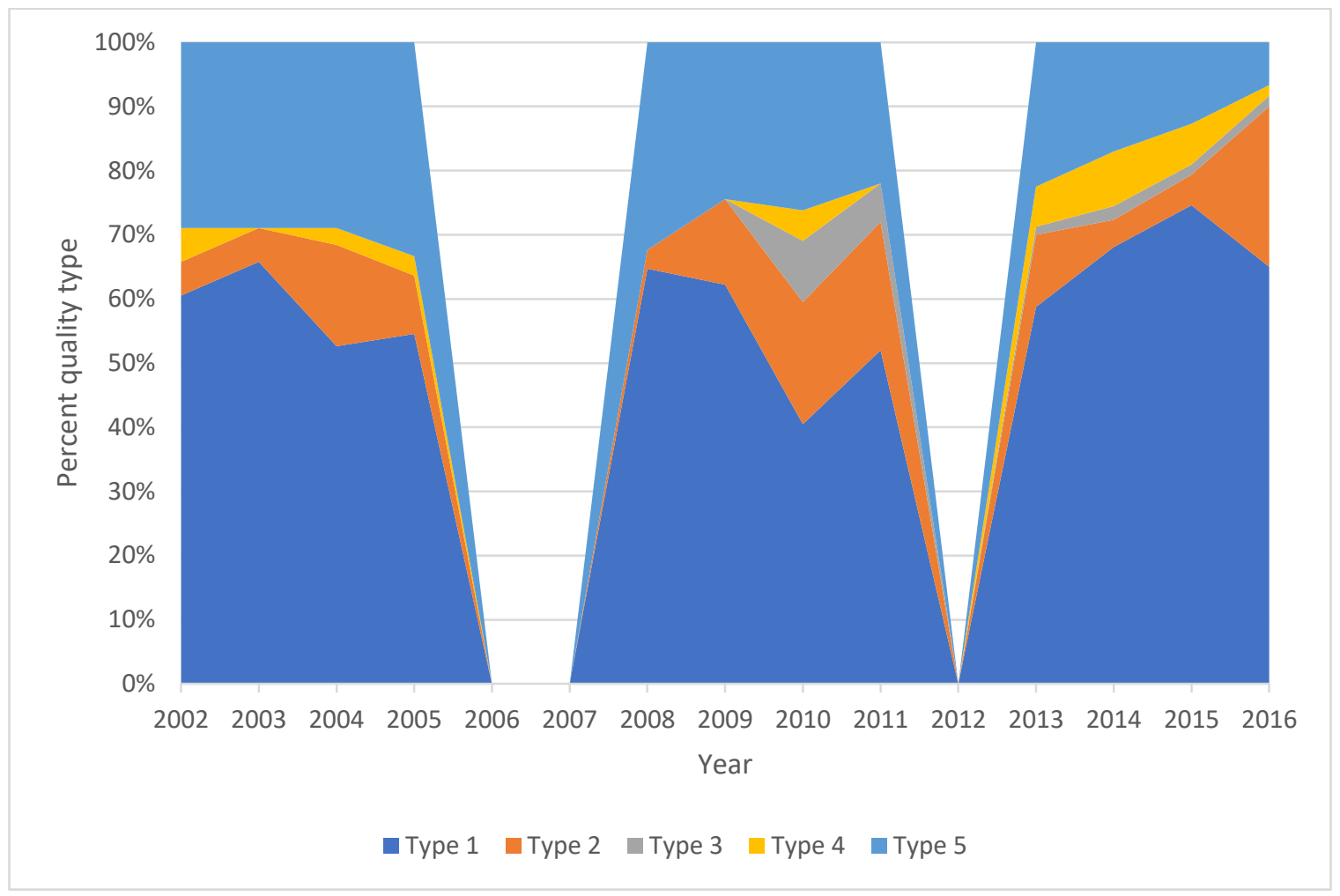

Figure B. 23: Quality composition of disclosure from I-Syngenta from 2002-2016. Type $1(p=0.573692)$, Type 2 ( $p=0.305055)$, Type $3(p=0.215392)$, Type $4(p=0.239045)$, Type 5 ( $p=0.11977)$.

Table B. 66: Regression statistics for the proportion of quality type 1 of CEDs from I-Syngenta from 20022016

\begin{tabular}{lr}
\hline \multicolumn{2}{c}{ Regression Statistics } \\
\hline Multiple R & 0.158063 \\
R Square & 0.024984
\end{tabular}




\begin{tabular}{|c|c|c|c|c|c|}
\hline Adjusted R & & & & & \\
\hline Square & -0.05002 & & & & \\
\hline Standard Error & 26.72003 & & & & \\
\hline Observations & 15 & & & & \\
\hline ANOVA & & & & & \\
\hline & & & & & Significance \\
\hline & $d f$ & SS & $M S$ & $F$ & $F$ \\
\hline Regression & 1 & 237.8283 & 237.8283 & 0.333112 & 0.573692 \\
\hline Residual & 13 & 9281.479 & 713.96 & & \\
\hline Total & 14 & 9519.308 & & & \\
\hline
\end{tabular}

Table B. 67: Regression statistics for the proportion of quality type 2 of CEDs from I-Syngenta from 20022016

\begin{tabular}{|c|c|c|c|c|c|}
\hline \multicolumn{2}{|c|}{ Regression Statistics } & & & & \\
\hline Multiple R & 0.283954 & & & & \\
\hline R Square & 0.08063 & & & & \\
\hline Adjusted R & & & & & \\
\hline Square & 0.009909 & & & & \\
\hline Standard Error & 7.960019 & & & & \\
\hline Observations & 15 & & & & \\
\hline \multicolumn{6}{|l|}{ ANOVA } \\
\hline & & & & & Significance \\
\hline & $d f$ & SS & $M S$ & $F$ & $F$ \\
\hline Regression & 1 & 72.24005 & 72.24005 & 1.140118 & 0.305055 \\
\hline Residual & 13 & 823.7047 & 63.3619 & & \\
\hline Total & 14 & 895.9448 & & & \\
\hline
\end{tabular}

Table B. 68: Regression statistics for the proportion of quality type 3 of CEDs from I-Syngenta from 20022016

\begin{tabular}{lr}
\hline \multicolumn{2}{c}{ Regression Statistics } \\
\hline Multiple R & 0.339728 \\
R Square & 0.115415 \\
Adjusted R & \\
Square & 0.04737
\end{tabular}




\begin{tabular}{|c|c|c|c|c|c|}
\hline Standard Error & 677609 & & & & \\
\hline Observations & 15 & & & & \\
\hline \multicolumn{6}{|l|}{ ANOVA } \\
\hline & & & & & Significance \\
\hline & $d f$ & SS & MS & $F$ & $F$ \\
\hline Regression & 1 & 12.1608 & 12.1608 & 1.696164 & 0.215392 \\
\hline Residual & 13 & 93.20466 & 7.169589 & & \\
\hline Total & 14 & 105.3655 & & & \\
\hline
\end{tabular}

Table B. 69: Regression statistics for the proportion of quality type 4 of CEDs from I-Syngenta from 20022016

\begin{tabular}{|c|c|c|c|c|c|}
\hline \multicolumn{2}{|c|}{ Regression Statistics } & & & & \\
\hline Multiple R & 0.323811 & & & & \\
\hline $\begin{array}{l}\text { R Square } \\
\text { Adjusted R }\end{array}$ & 0.104854 & & & & \\
\hline Square & 0.035996 & & & & \\
\hline Standard Error & 2.903589 & & & & \\
\hline Observations & 15 & & & & \\
\hline \multicolumn{6}{|l|}{ ANOVA } \\
\hline & $d f$ & SS & MS & $F$ & $\begin{array}{c}\text { Significance } \\
F\end{array}$ \\
\hline Regression & 1 & 12.8382 & 12.8382 & 1.522768 & 0.239045 \\
\hline Residual & 13 & 109.6008 & 8.430832 & & \\
\hline Total & 14 & 122.439 & & & \\
\hline
\end{tabular}

Table B. 70: Regression statistics for the proportion of quality type 5 of CEDs from I-Syngenta from 20022016

\begin{tabular}{lr}
\hline \multicolumn{2}{c}{ Regression Statistics } \\
\hline Multiple R & 0.419291 \\
R Square & 0.175805 \\
Adjusted R & \\
Square & 0.112406 \\
Standard Error & 11.43335
\end{tabular}




\begin{tabular}{lrrrcc} 
ANOVA & & & & & \multicolumn{2}{c}{ Significance } \\
& $d f$ & \multicolumn{1}{c}{ SS } & MS & \multicolumn{1}{c}{$F$} & \multicolumn{1}{c}{$F$} \\
\hline Regression & 1 & 362.4869 & 362.4869 & 2.77297 & 0.11977 \\
Residual & 13 & 1699.38 & 130.7215 & & \\
Total & 14 & 2061.867 & & & \\
\hline
\end{tabular}

B.3.9 Quality analysis of P-BASF

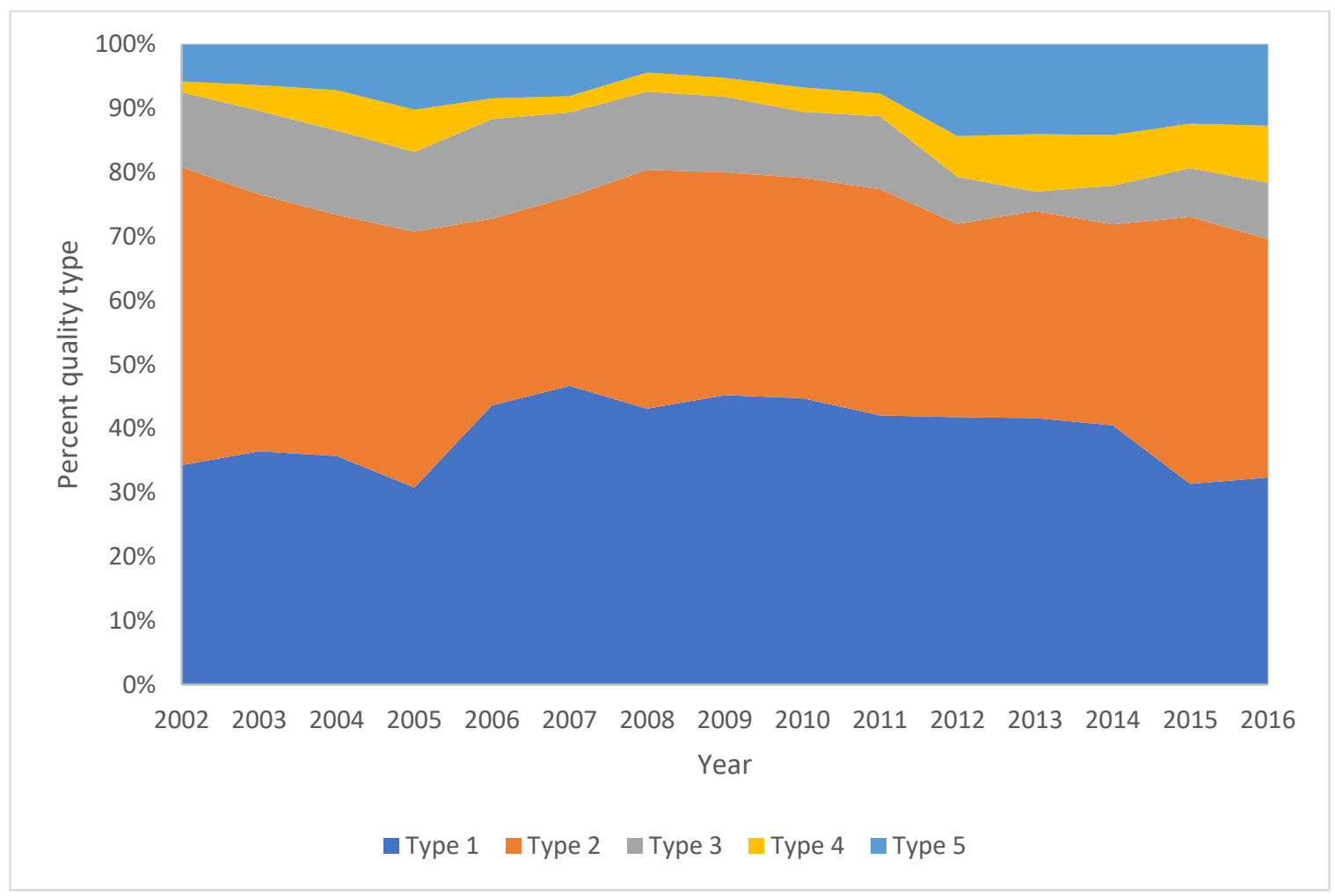

Figure B. 24: Quality composition of disclosure from P-BASF from 2002-2016. Type 1 ( $p=0.914266)$, Type $2(p=0.213162)$, Type $3(p=0.00116)$, Type $4(p=0.009394)$, Type $5(p=0.00334)$.

Table B. 71: Regression statistics for the proportion of quality type 1 of CEDs from P-BASF from 20022016

\begin{tabular}{lr}
\hline \multicolumn{2}{c}{ Regression Statistics } \\
\hline Multiple R & 0.030432 \\
R Square & 0.000926
\end{tabular}




\begin{tabular}{|c|c|c|c|c|c|}
\hline Adjusted R & & & & & \\
\hline Square & -0.07593 & & & & \\
\hline Standard Error & 5.56087 & & & & \\
\hline Observations & 15 & & & & \\
\hline ANOVA & & & & & \\
\hline & & & & & Significance \\
\hline & $d f$ & SS & MS & $F$ & $F$ \\
\hline Regression & 1 & 0.372631 & 0.372631 & 0.01205 & 0.914266 \\
\hline Residual & 13 & 402.0026 & 30.92328 & & \\
\hline Total & 14 & 402.3753 & & & \\
\hline
\end{tabular}

Table B. 72: Regression statistics for the proportion of quality type 2 of CEDs from P-BASF from 20022016

\begin{tabular}{|c|c|c|c|c|c|}
\hline \multicolumn{2}{|c|}{ Regression Statistics } & & & & \\
\hline Multiple R & 0.341285 & & & & \\
\hline $\begin{array}{l}\text { R Square } \\
\text { Adjusted R }\end{array}$ & 0.116475 & & & & \\
\hline Square & 0.048512 & & & & \\
\hline Standard Error & 4.827269 & & & & \\
\hline Observations & 15 & & & & \\
\hline \multicolumn{6}{|l|}{ ANOVA } \\
\hline & $d f$ & SS & MS & $F$ & $\begin{array}{c}\text { Significance } \\
F \\
\end{array}$ \\
\hline Regression & 1 & 39.93571 & 39.93571 & 1.713793 & 0.213162 \\
\hline Residual & 13 & 302.9328 & 23.30252 & & \\
\hline Total & 14 & 342.8685 & & & \\
\hline
\end{tabular}

Table B. 73: Regression statistics for the proportion of quality type 3 of CEDs from P-BASF from 20022016

\begin{tabular}{lr}
\hline \multicolumn{2}{c}{ Regression Statistics } \\
\hline Multiple R & 0.754187 \\
R Square & 0.568798
\end{tabular}


Adjusted R

\begin{tabular}{|c|c|c|c|c|c|}
\hline Square & 0.535628 & & & & \\
\hline Standard Error & 2.258385 & & & & \\
\hline Observations & 15 & & & & \\
\hline \multicolumn{6}{|l|}{ ANOVA } \\
\hline & & & & & Significance \\
\hline & $d f$ & SS & MS & $F$ & $F$ \\
\hline Regression & 1 & 87.46126 & 87.46126 & 17.14826 & 0.00116 \\
\hline Residual & 13 & 66.30391 & 5.100301 & & \\
\hline Total & 14 & 153.7652 & & & \\
\hline
\end{tabular}

Table B. 74: Regression statistics for the proportion of quality type 4 of CEDs from P-BASF from 20022016

\begin{tabular}{lr}
\hline \multicolumn{2}{c}{ Regression Statistics } \\
\hline Multiple R & 0.64519 \\
R Square & 0.41627 \\
Adjusted R & \\
Square & 0.371368 \\
Standard Error & 1.925765 \\
Observations & 15 \\
\hline
\end{tabular}

ANOVA

\begin{tabular}{lrrrcc}
\hline & & & & & \multicolumn{1}{c}{ Significance } \\
& $d f$ & \multicolumn{1}{c}{ SS } & MS & \multicolumn{1}{c}{$F$} & \multicolumn{1}{c}{$F$} \\
\hline Regression & 1 & 34.38055 & 34.38055 & 9.270571 & 0.009394 \\
Residual & 13 & 48.2114 & 3.708569 & & \\
Total & 14 & 82.59195 & & & \\
\hline
\end{tabular}

Table B. 75: Regression statistics for the proportion of quality type 5 of CEDs from P-BASF from 20022016

\begin{tabular}{lr}
\hline \multicolumn{2}{c}{ Regression Statistics } \\
\hline Multiple R & 0.704865 \\
R Square & 0.496834 \\
Adjusted R & \\
Square & 0.458129 \\
Standard Error & 2.567163
\end{tabular}




\begin{tabular}{lrcccc} 
ANOVA & & & & & \multicolumn{2}{c}{ Significance } \\
& $d f$ & \multicolumn{1}{c}{ SS } & MS & \multicolumn{1}{c}{$F$} & \multicolumn{1}{c}{} \\
\hline Regression & 1 & 84.59613 & 84.59613 & 12.83641 & 0.00334 \\
Residual & 13 & 85.67424 & 6.590327 & & \\
Total & 14 & 170.2704 & & & \\
\hline
\end{tabular}

B.3.10 Quality analysis of P-Bayer

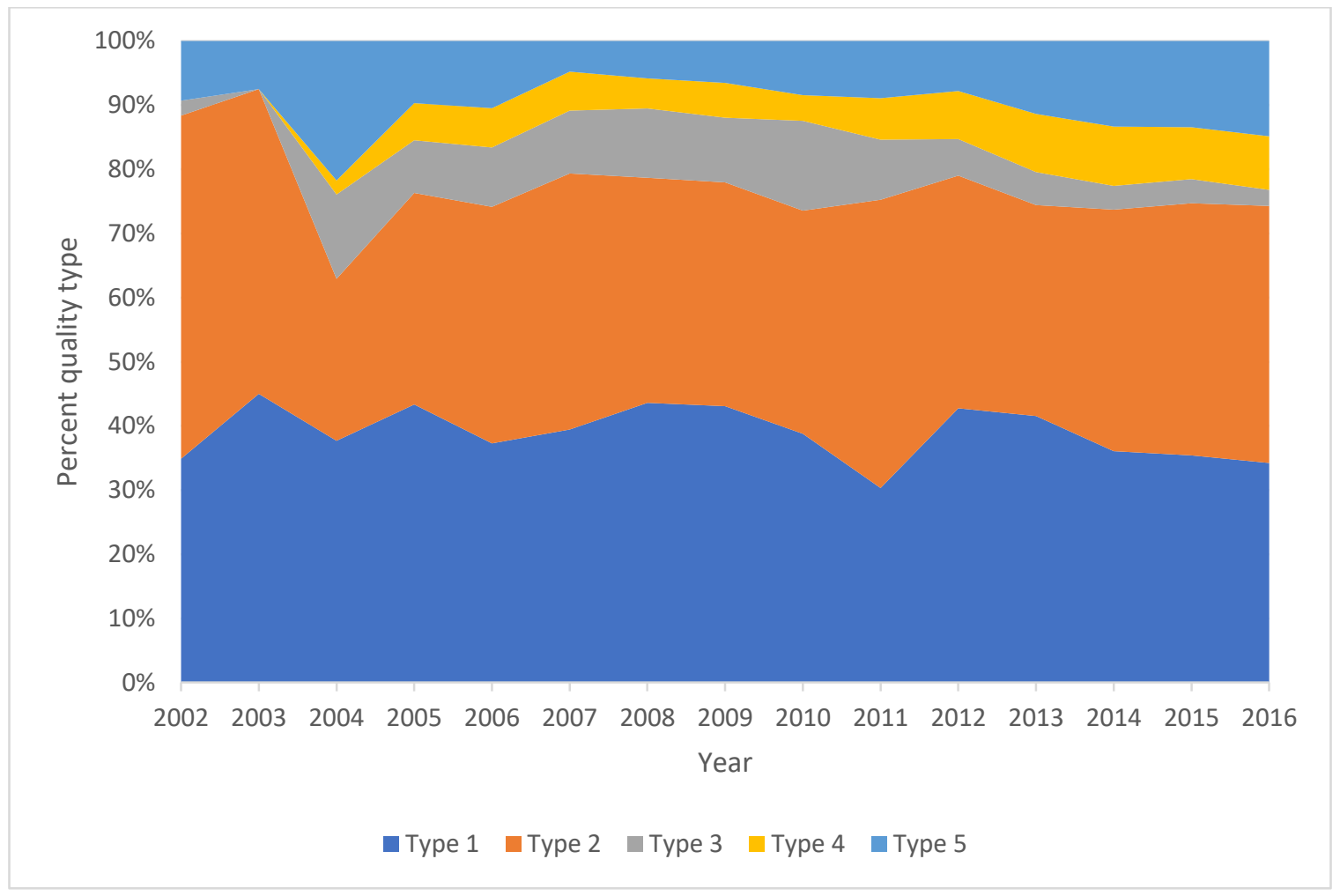

Figure B. 25: Quality composition of disclosure from P-Bayer from 2002-2016. Type 1 ( $p=0.25242)$, Type 2 $(p=0.534258)$, Type 3 ( $p=0.55123)$, Type $4(p=3.16 E-05)$, Type $5(p=0.576412)$.

Table B. : Regression statistics for the proportion of quality type 1 of CEDs from P-Bayer from 2002-2016

\begin{tabular}{lr}
\hline \multicolumn{2}{c}{ Regression Statistics } \\
\hline Multiple R & 0.315242 \\
R Square & 0.099377 \\
Adjusted R & \\
Square & 0.030099 \\
Standard Error & 4.198206
\end{tabular}




\begin{tabular}{|c|c|c|c|c|c|}
\hline Observations & 15 & & & & \\
\hline \multicolumn{6}{|l|}{ ANOVA } \\
\hline & & & & & Significance \\
\hline & $d f$ & SS & MS & $F$ & $F$ \\
\hline Regression & 1 & 25.28226 & 25.28226 & 1.43446 & 0.25242 \\
\hline Residual & 13 & 229.1241 & 17.62493 & & \\
\hline Total & 14 & 254.4064 & & & \\
\hline
\end{tabular}

Table B. 76: Regression statistics for the proportion of quality type 2 of CEDs from P-Bayer from 20022016

\begin{tabular}{|c|c|c|c|c|c|}
\hline \multicolumn{2}{|c|}{ Regression Statistics } & & & & \\
\hline Multiple R & 0.174366 & & & & \\
\hline R Square & 0.030403 & & & & \\
\hline Adjusted R & & & & & \\
\hline Square & -0.04418 & & & & \\
\hline Standard Error & 6.88048 & & & & \\
\hline Observations & 15 & & & & \\
\hline \multicolumn{6}{|l|}{ ANOVA } \\
\hline & $d f$ & SS & MS & $F$ & $\begin{array}{c}\text { Significance } \\
F\end{array}$ \\
\hline Regression & 1 & 19.29797 & 19.29797 & 0.407637 & 0.534258 \\
\hline Residual & 13 & 615.433 & 47.341 & & \\
\hline Total & 14 & 634.731 & & & \\
\hline
\end{tabular}

Table B. 77: Regression statistics for the proportion of quality type 3 of CEDs from P-Bayer from 20022016

\begin{tabular}{lr}
\hline \multicolumn{2}{c}{ Regression Statistics } \\
\hline Multiple R & 0.167287 \\
R Square & 0.027985 \\
Adjusted R & \\
Square & -0.04679 \\
Standard Error & 4.304475 \\
Observations & 15 \\
\hline
\end{tabular}


ANOVA

\begin{tabular}{lrcccc}
\hline & & & & \multicolumn{2}{c}{ Significance } \\
& $d f$ & SS & MS & $F$ & \multicolumn{1}{c}{$F$} \\
\hline Regression & 1 & 6.934859 & 6.934859 & 0.37428 & 0.55123 \\
Residual & 13 & 240.8706 & 18.52851 & & \\
Total & 14 & 247.8055 & & & \\
\hline
\end{tabular}

Table B. 78: Regression statistics for the proportion of quality type 4 of CEDs from P-Bayer from 20022016

\begin{tabular}{|c|c|c|c|c|c|}
\hline \multicolumn{2}{|c|}{ Regression Statistics } & & & & \\
\hline Multiple R & 0.864826 & & & & \\
\hline $\begin{array}{l}\text { R Square } \\
\text { Adjusted R }\end{array}$ & 0.747923 & & & & \\
\hline Square & 0.728533 & & & & \\
\hline Standard Error & 1.535661 & & & & \\
\hline Observations & 15 & & & & \\
\hline \multicolumn{6}{|l|}{ ANOVA } \\
\hline & & & & & Significance \\
\hline & $d f$ & SS & $M S$ & $F$ & $F$ \\
\hline Regression & 1 & 90.96171 & 90.96171 & 38.57162 & $3.16 \mathrm{E}-05$ \\
\hline Residual & 13 & 30.65731 & 2.358255 & & \\
\hline Total & 14 & 121.619 & & & \\
\hline
\end{tabular}

Table B. 79: Regression statistics for the proportion of quality type 5 of CEDs from P-Bayer from 20022016

\begin{tabular}{lr}
\hline \multicolumn{2}{c}{ Regression Statistics } \\
\hline Multiple R & 0.156956 \\
R Square & 0.024635 \\
Adjusted R & \\
Square & -0.05039 \\
Standard Error & 4.392755 \\
Observations & 15 \\
\hline
\end{tabular}




\begin{tabular}{|c|c|c|c|c|c|}
\hline & $d f$ & SS & $M S$ & $F$ & $\begin{array}{c}\text { Significance } \\
F\end{array}$ \\
\hline Regression & 1 & 6.335886 & 6.335886 & 0.328347 & 0.576412 \\
\hline Residual & 13 & 250.8518 & 19.29629 & & \\
\hline Total & 14 & 257.1877 & & & \\
\hline
\end{tabular}

\section{B.3.11 P-Monsanto}

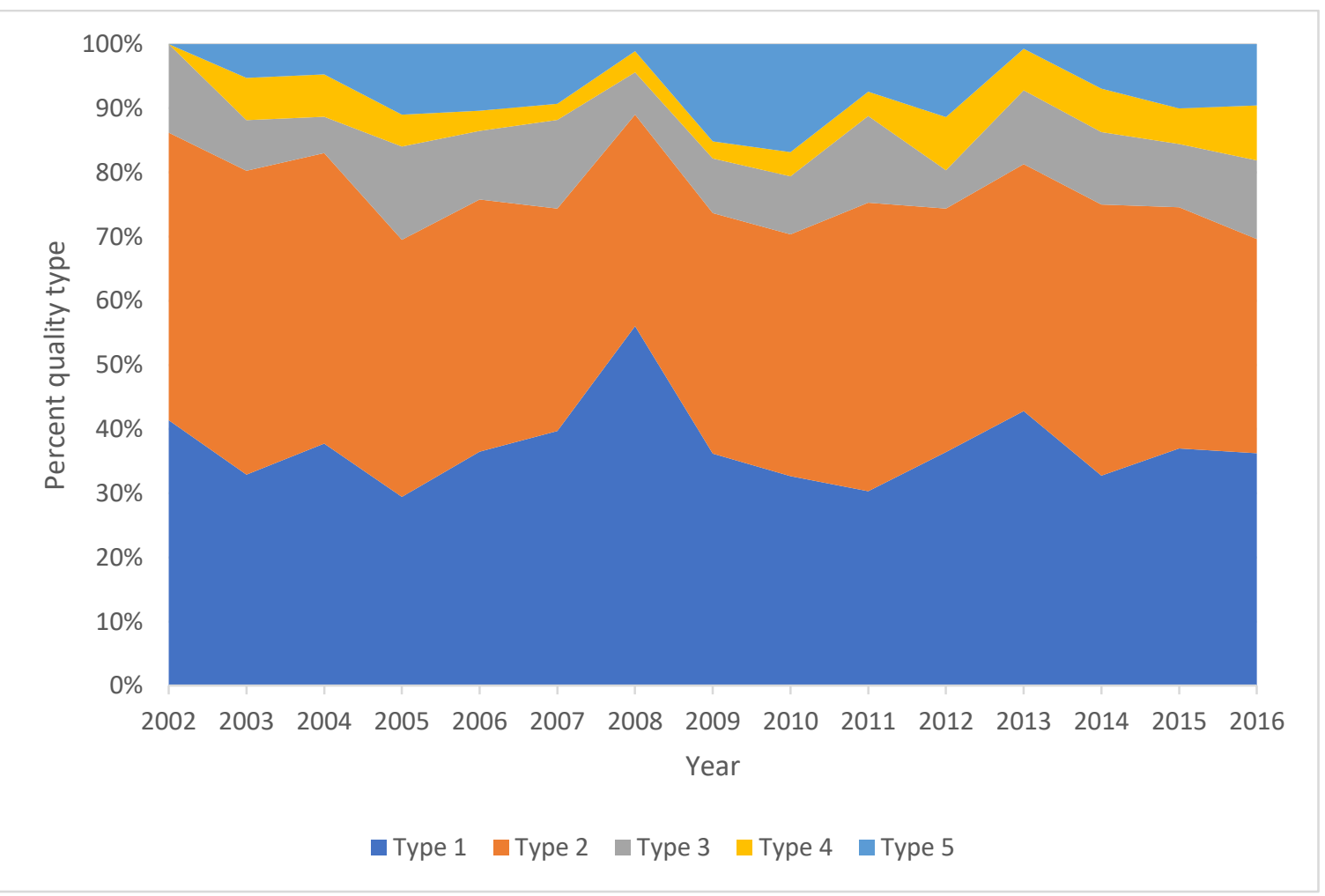

Figure B. 26: Quality composition of disclosure from P-Monsanto from 2002-2016. Type 1 ( $p=0.82403)$, Type 2 ( $p=0.0595)$, Type $3(p=0.926843)$, Type 4 ( $p=0.042729)$, Type $5(p=0.354159)$.

Table B. 80: Regression statistics for the proportion of quality type 1 of CEDs from P-Monsanto from 2002-2016

\begin{tabular}{lr}
\hline \multicolumn{2}{c}{ Regression Statistics } \\
\hline Multiple R & 0.062807 \\
R Square & 0.003945 \\
Adjusted R & \\
Square & -0.07267 \\
Standard Error & 6.668856 \\
Observations & 15 \\
\hline
\end{tabular}


ANOVA

\begin{tabular}{lrrccc}
\hline & & & & \multicolumn{2}{c}{ Significance } \\
& $d f$ & \multicolumn{1}{c}{ SS } & MS & \multicolumn{1}{c}{$F$} & \multicolumn{1}{c}{$F$} \\
\hline Regression & 1 & 2.289681 & 2.289681 & 0.051484 & 0.82403 \\
Residual & 13 & 578.1573 & 44.47364 & & \\
Total & 14 & 580.447 & & & \\
\hline
\end{tabular}

Table B. 81: Regression statistics for the proportion of quality type 2 of CEDs from P-Monsanto from 2002-2016

\begin{tabular}{|c|c|c|c|c|c|}
\hline \multicolumn{2}{|c|}{ Regression Statistics } & & & & \\
\hline Multiple R & 0.496929 & & & & \\
\hline $\begin{array}{l}\text { R Square } \\
\text { Adjusted R }\end{array}$ & 0.246938 & & & & \\
\hline Square & 0.18901 & & & & \\
\hline Standard Error & 4.021525 & & & & \\
\hline Observations & 15 & & & & \\
\hline \multicolumn{6}{|l|}{ ANOVA } \\
\hline & $d f$ & SS & MS & $F$ & $\begin{array}{c}\text { Significance } \\
F\end{array}$ \\
\hline Regression & 1 & 68.94173 & 68.94173 & 4.262856 & 0.0595 \\
\hline Residual & 13 & 210.2446 & 16.17266 & & \\
\hline Total & 14 & 279.1863 & & & \\
\hline
\end{tabular}

Table B. 82: Regression statistics for the proportion of quality type 3 of CEDs from P-Monsanto from 2002-2016

\begin{tabular}{lr}
\hline \multicolumn{2}{c}{ Regression Statistics } \\
\hline Multiple R & 0.025955 \\
R Square & 0.000674 \\
Adjusted R & \\
Square & -0.0762
\end{tabular}




\begin{tabular}{|c|c|c|c|c|c|}
\hline Standard Error & 3.08808 & & & & \\
\hline Observations & 15 & & & & \\
\hline \multicolumn{6}{|l|}{ ANOVA } \\
\hline & & & & & Significance \\
\hline & $d f$ & SS & $M S$ & $F$ & $F$ \\
\hline Regression & 1 & 0.083571 & 0.083571 & 0.008764 & 0.926843 \\
\hline Residual & 13 & 123.9711 & 9.536236 & & \\
\hline Total & 14 & 124.0546 & & & \\
\hline
\end{tabular}

Table B. 83: Regression statistics for the proportion of quality type 4 of CEDs from P-Monsanto from 2002-2016

\begin{tabular}{|c|c|c|c|c|c|}
\hline \multicolumn{2}{|c|}{ Regression Statistics } & & & & \\
\hline Multiple R & 0.528718 & & & & \\
\hline $\begin{array}{l}\text { R Square } \\
\text { Adiusted R }\end{array}$ & 0.279543 & & & & \\
\hline Square & 0.224123 & & & & \\
\hline Standard Error & 2.103783 & & & & \\
\hline Observations & 15 & & & & \\
\hline \multicolumn{6}{|l|}{ ANOVA } \\
\hline & $d f$ & SS & MS & $F$ & $\begin{array}{c}\text { Significance } \\
F\end{array}$ \\
\hline Regression & 1 & 22.32469 & 22.32469 & 5.044098 & 0.042729 \\
\hline Residual & 13 & 57.53674 & 4.425903 & & \\
\hline Total & 14 & 79.86143 & & & \\
\hline
\end{tabular}

Table B. 84: Regression statistics for the proportion of quality type 5 of CEDs from P-Monsanto from 2002-2016

\begin{tabular}{lr}
\hline \multicolumn{2}{c}{ Regression Statistics } \\
\hline Multiple R & 0.257505 \\
R Square & 0.066309 \\
Adjusted R & \\
Square & -0.00551 \\
Standard Error & 4.997967 \\
Observations & 15 \\
\hline
\end{tabular}

ANOVA 


\begin{tabular}{lrcccc}
\hline & & & & & \multicolumn{2}{c}{ Significance } \\
& $d f$ & SS & MS & $F$ & $F$ \\
\hline Regression & 1 & 23.06206 & 23.06206 & 0.923233 & 0.354159 \\
Residual & 13 & 324.7357 & 24.97967 & & \\
Total & 14 & 347.7978 & & & \\
\hline
\end{tabular}

B.3.12 Quality analysis of P-Syngenta

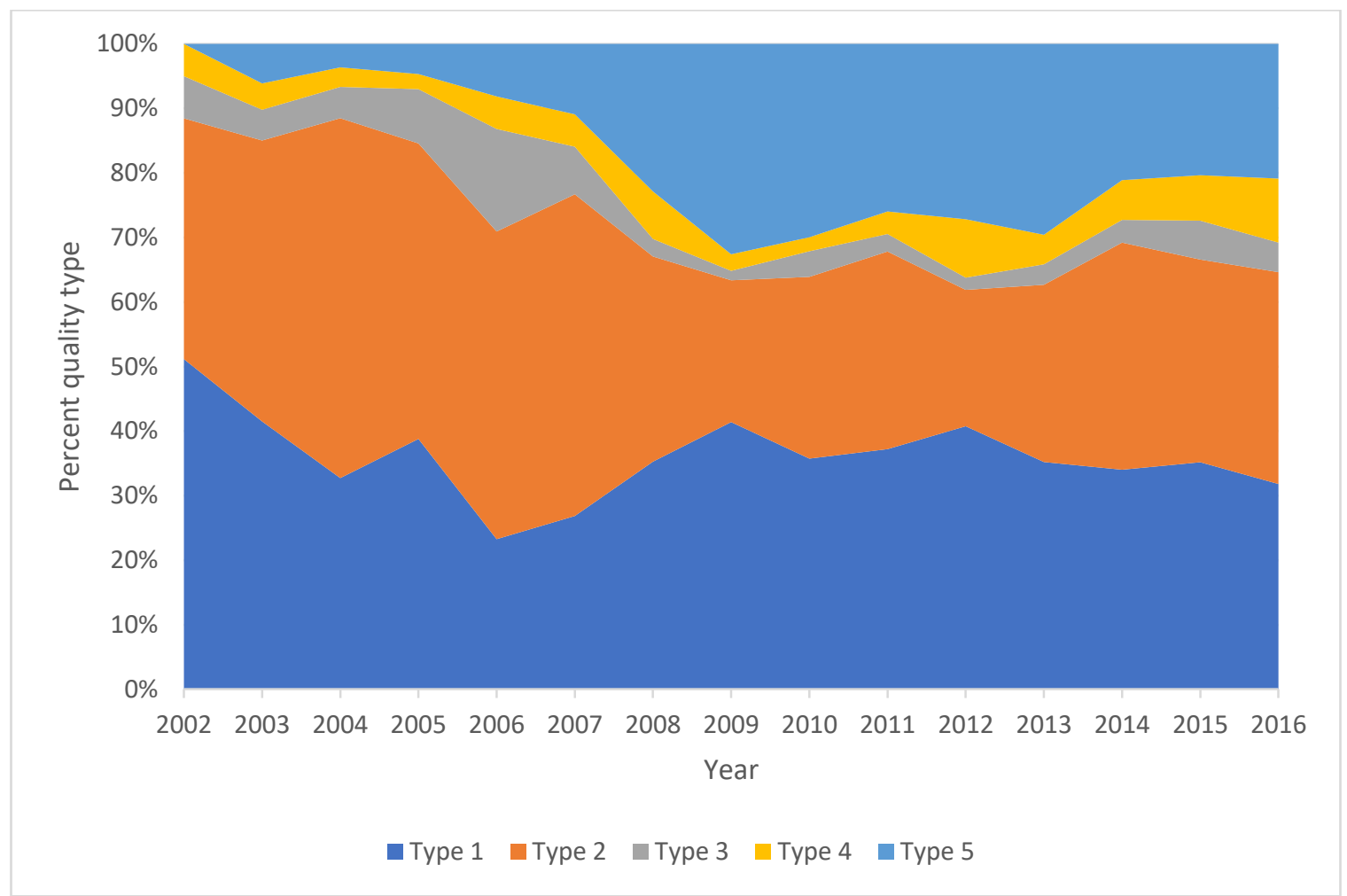

Figure B. 27: Quality composition of disclosure from P-Syngenta from 2002-2016. Type 1( $p=0.345442)$, Type $2(p=0.013754)$, Type $3(p=0.168384)$, Type $4(p=0.038067)$, Type $5(p=0.001349)$.

Table B. 85: Regression statistics for the proportion of quality type 1 of CEDs from P-Syngenta from 20022016

\begin{tabular}{lr}
\hline \multicolumn{2}{c}{ Regression Statistics } \\
\hline Multiple R & 0.262041 \\
R Square & 0.068666 \\
Adjusted R & \\
Square & -0.00298 \\
Standard Error & 6.562176 \\
Observations & 15 \\
\hline
\end{tabular}

ANOVA

Significance

\begin{tabular}{lllll}
$d f$ & $S S$ & $M S$ & $F$ & $F$ \\
\hline
\end{tabular}




\begin{tabular}{lrrrrr} 
Regression & 1 & 41.27361 & 41.27361 & 0.958466 & 0.345442 \\
Residual & 13 & 559.808 & 43.06215 & & \\
Total & 14 & 601.0816 & & & \\
\hline
\end{tabular}

Table B. 86: Regression statistics for the proportion of quality type 2 of CEDs from P-Syngenta from 20022016

\begin{tabular}{|c|c|c|c|c|c|}
\hline \multicolumn{2}{|c|}{ Regression Statistics } & & & & \\
\hline Multiple R & 0.61962 & & & & \\
\hline R Square & 0.383929 & & & & \\
\hline Adjusted R & & & & & \\
\hline Square & 0.336538 & & & & \\
\hline Standard Error & 8.441169 & & & & \\
\hline Observations & 15 & & & & \\
\hline \multicolumn{6}{|l|}{ ANOVA } \\
\hline & & & & & Significance \\
\hline & $d f$ & SS & $M S$ & $F$ & $F$ \\
\hline Regression & 1 & 577.2553 & 577.2553 & 8.10145 & 0.013754 \\
\hline Residual & 13 & 926.2933 & 71.25333 & & \\
\hline Total & 14 & 1503.549 & & & \\
\hline
\end{tabular}

Table B. 87: Regression statistics for the proportion of quality type 3 of CEDs from P-Syngenta from 20022016

\begin{tabular}{lr}
\hline \multicolumn{2}{c}{ Regression Statistics } \\
\hline Multiple R & 0.375039 \\
R Square & 0.140655 \\
Adjusted R & \\
Square & 0.074551 \\
Standard Error & 3.432948 \\
Observations & 15 \\
\hline
\end{tabular}

\begin{tabular}{|c|c|c|c|c|c|}
\hline & & & & & Significance \\
\hline & $d f$ & SS & MS & $F$ & $F$ \\
\hline Regression & & 25.07632 & 25.07632 & 2.127793 & 0.168384 \\
\hline
\end{tabular}




\begin{tabular}{lrrr} 
Residual & 13 & 153.2067 & 11.78513 \\
Total & 14 & 178.283 & \\
\hline
\end{tabular}

Table B. 88: Regression statistics for the proportion of quality type 4 of CEDs from P-Syngenta from 20022016

\begin{tabular}{|c|c|c|c|c|c|}
\hline \multicolumn{2}{|c|}{ Regression Statistics } & & & & \\
\hline Multiple R & 0.53918 & & & & \\
\hline $\begin{array}{l}\text { R Square } \\
\text { Adjusted R }\end{array}$ & 0.290715 & & & & \\
\hline Square & 0.236155 & & & & \\
\hline Standard Error & 2.086543 & & & & \\
\hline Observations & 15 & & & & \\
\hline \multicolumn{6}{|l|}{ ANOVA } \\
\hline & & & & & Significance \\
\hline & $d f$ & SS & $M S$ & $F$ & $F$ \\
\hline Regression & 1 & 23.19769 & 23.19769 & 5.328317 & 0.038067 \\
\hline Residual & 13 & 56.59759 & 4.353661 & & \\
\hline Total & 14 & 79.79528 & & & \\
\hline
\end{tabular}

Table B. 89: Regression statistics for the proportion of quality type 5 of CEDs from P-Syngenta from 20022016

\begin{tabular}{|c|c|c|c|c|c|}
\hline \multicolumn{2}{|c|}{ Regression Statistics } & & & & \\
\hline Multiple R & 0.747786 & & & & \\
\hline $\begin{array}{l}\text { R Square } \\
\text { Adjusted R }\end{array}$ & 0.559184 & & & & \\
\hline Square & 0.525275 & & & & \\
\hline Standard Error & 7.545606 & & & & \\
\hline Observations & 15 & & & & \\
\hline \multicolumn{6}{|l|}{ ANOVA } \\
\hline & $d f$ & SS & MS & $F$ & $\begin{array}{c}\text { Significance } \\
F\end{array}$ \\
\hline Regression & 1 & 938.9212 & 938.9212 & 16.49077 & 0.001349 \\
\hline Residual & 13 & 740.1702 & 56.93617 & & \\
\hline
\end{tabular}




\section{B.4 Coder Reliability}

Results from coder reliability which was calculated using statistics calculator Dfreelon online statistics calculator.

Table B. 90: Intra-Coder reliability

\begin{tabular}{|c|c|c|c|c|c|c|c|c|}
\hline & \begin{tabular}{|l|} 
Percent \\
Agreement
\end{tabular} & Scott's Pi & Cohen's Kappa & $\begin{array}{l}\text { Krippendorff's } \\
\text { Alpha }\end{array}$ & N Agreements & \begin{tabular}{|l|}
$\mathrm{N}$ \\
Disagreements
\end{tabular} & N Cases & N Decisions \\
\hline Variable 1 (cols 1 \& 2) & 100 & 1 & 1 & 1 & 6 & 0 & 6 & 12 \\
\hline Variable 2 (cols 3 \& 4) & 83.33333 & 0.555556 & 0.571429 & 0.592593 & 5 & 1 & 6 & 12 \\
\hline Variable 3 (cols 5 \& 6) & 100 & 1 & 1 & 1 & 6 & 0 & 6 & 12 \\
\hline Variable 4 (cols 7 \& 8) & 100 & 1 & 1 & 1 & 6 & 0 & 6 & 12 \\
\hline Variable 5 (cols 9 \& 10) & 100 & 1 & 1 & 1 & 6 & 0 & 6 & 12 \\
\hline Variable 6 (cols $11 \& 12$ ) & 83.33333 & 0.675676 & 0.684211 & 0.702703 & 5 & 1 & 6 & 12 \\
\hline Variable 7 (cols 13 \& 14) & 100 & 1 & 1 & 1 & 6 & 0 & 6 & 12 \\
\hline Variable 8 (cols 15 \& 16) & 100 & 1 & 1 & 1 & 6 & 0 & 6 & 12 \\
\hline Variable 9 (cols $17 \& 18$ ) & 83.33333 & -0.09091 & 6.66E-16 & 0 & 5 & 1 & 6 & 12 \\
\hline $\begin{array}{l}\text { Variable } 10 \text { (cols } 19 \& \\
20)\end{array}$ & 100 & 1 & 1 & 1 & 6 & 0 & 6 & 12 \\
\hline $\begin{array}{l}\text { Variable } 11 \text { (cols } 21 \& \\
22 \text { ) }\end{array}$ & 100 & 1 & 1 & 1 & 6 & 0 & 6 & 12 \\
\hline $\begin{array}{l}\text { Variable } 12 \text { (cols } 23 \& \\
24)\end{array}$ & 100 & 1 & 1 & 1 & 6 & 0 & 6 & 12 \\
\hline $\begin{array}{l}\text { Variable } 13 \text { (cols } 25 \& \\
26 \text { ) }\end{array}$ & 83.33333 & -0.09091 & $6.66 \mathrm{E}-16$ & 0 & 5 & 1 & 6 & 12 \\
\hline $\begin{array}{l}\text { Variable } 14 \text { (cols } 27 \& \\
28 \text { ) }\end{array}$ & 100 & 1 & 1 & 1 & 6 & 0 & 6 & 12 \\
\hline $\begin{array}{l}\text { Variable } 15 \text { (cols } 29 \& \\
30)\end{array}$ & 100 & 1 & 1 & 1 & 6 & 0 & 6 & 12 \\
\hline $\begin{array}{l}\text { Variable } 16 \text { (cols } 31 \& \\
32 \text { ) }\end{array}$ & 83.33333 & -0.09091 & $6.66 \mathrm{E}-16$ & 0 & 5 & 1 & 6 & 12 \\
\hline
\end{tabular}




\begin{tabular}{|c|c|c|c|c|c|c|c|c|}
\hline & \begin{tabular}{|l} 
Percent \\
Agreement
\end{tabular} & Scott's Pi & Cohen's Kappa & $\begin{array}{l}\text { Krippendorff's } \\
\text { Alpha }\end{array}$ & N Agreements & \begin{tabular}{|l|l|}
$\mathrm{N}$ \\
Disagreements
\end{tabular} & N Cases & N Decisions \\
\hline $\begin{array}{l}\text { Variable } 17 \text { (cols } 33 \text { \& } \\
\text { 34) }\end{array}$ & 100 & 1 & 1 & 1 & 6 & 0 & 6 & 12 \\
\hline $\begin{array}{l}\text { Variable } 18 \text { (cols } 35 \text { \& } \\
36 \text { ) }\end{array}$ & 100 & 1 & 1 & 1 & 6 & 0 & 6 & 12 \\
\hline $\begin{array}{l}\text { Variable } 19 \text { (cols } 37 \& \\
38 \text { ) }\end{array}$ & 100 & 1 & 1 & 1 & 6 & 0 & 6 & 12 \\
\hline $\begin{array}{l}\text { Variable } 20 \text { (cols } 39 \& \\
40 \text { ) }\end{array}$ & 100 & 1 & 1 & 1 & 6 & 0 & 6 & 12 \\
\hline $\begin{array}{l}\text { Variable } 21 \text { (cols } 41 \& \\
42 \text { ) }\end{array}$ & 100 & 1 & 1 & 1 & 6 & 0 & 6 & 12 \\
\hline $\begin{array}{l}\text { Variable } 22 \text { (cols } 43 \& \\
44 \text { ) }\end{array}$ & 83.33333 & 0.755102 & 0.76 & 0.77551 & 5 & 1 & 6 & 12 \\
\hline $\begin{array}{l}\text { Variable } 23 \text { (cols } 45 \& \\
46 \text { ) }\end{array}$ & 83.33333 & -0.09091 & $6.66 \mathrm{E}-16$ & 0 & 5 & 1 & 6 & 12 \\
\hline $\begin{array}{l}\text { Variable } 24 \text { (cols } 47 \& \\
48 \text { ) }\end{array}$ & 100 & 1 & 1 & 1 & 6 & 0 & 6 & 12 \\
\hline $\begin{array}{l}\text { Variable } 25 \text { (cols } 49 \& \\
50 \text { ) }\end{array}$ & 100 & 1 & 1 & 1 & 6 & 0 & 6 & 12 \\
\hline $\begin{array}{l}\text { Variable } 26 \text { (cols } 51 \text { \& } \\
52 \text { ) }\end{array}$ & 100 & 1 & 1 & 1 & 6 & 0 & 6 & 12 \\
\hline $\begin{array}{l}\text { Variable } 27 \text { (cols } 53 \text { \& } \\
54 \text { ) }\end{array}$ & 100 & 1 & 1 & 1 & 6 & 0 & 6 & 12 \\
\hline $\begin{array}{l}\text { Variable } 28 \text { (cols } 55 \& \\
56 \text { ) }\end{array}$ & 83.33333 & 0.428571 & 0.454545 & 0.47619 & 5 & 1 & 6 & 12 \\
\hline $\begin{array}{l}\text { Variable } 29 \text { (cols } 57 \text { \& } \\
58 \text { ) }\end{array}$ & 83.33333 & 0.428571 & 0.454545 & 0.47619 & 5 & 1 & 6 & 12 \\
\hline $\begin{array}{l}\text { Variable } 30 \text { (cols } 59 \& \\
60 \text { ) }\end{array}$ & 83.33333 & 0.428571 & 0.454545 & 0.47619 & 5 & 1 & 6 & 12 \\
\hline $\begin{array}{l}\text { Variable } 31 \text { (cols } 61 \text { \& } \\
62 \text { ) }\end{array}$ & 100 & 1 & 1 & 1 & 6 & 0 & 6 & 12 \\
\hline $\begin{array}{l}\text { Variable } 32 \text { (cols } 63 \text { \& } \\
64 \text { ) }\end{array}$ & 100 & 1 & 1 & 1 & 6 & 0 & 6 & 12 \\
\hline $\begin{array}{l}\text { Variable } 33 \text { (cols } 65 \text { \& } \\
66 \text { ) }\end{array}$ & 100 & 1 & 1 & 1 & 6 & 0 & 6 & 12 \\
\hline Variable 34 (cols 67 \& & 100 & 1 & 1 & 1 & 6 & 0 & 6 & 12 \\
\hline
\end{tabular}




\begin{tabular}{|c|c|c|c|c|c|c|c|c|}
\hline & \begin{tabular}{|l|} 
Percent \\
Agreement
\end{tabular} & Scott's Pi & Cohen's Kappa & $\begin{array}{l}\text { Krippendorff's } \\
\text { Alpha }\end{array}$ & N Agreements & \begin{tabular}{|l|l|}
$\mathrm{N}$ \\
Disagreements
\end{tabular} & N Cases & N Decisions \\
\hline 68) & & & & & & & & \\
\hline $\begin{array}{l}\text { Variable } 35 \text { (cols } 69 \& \\
\text { 70) }\end{array}$ & 100 & 1 & 1 & 1 & 6 & 0 & 6 & 12 \\
\hline $\begin{array}{l}\text { Variable } 36 \text { (cols } 71 \& \\
72 \text { ) }\end{array}$ & 100 & 1 & 1 & 1 & 6 & 0 & 6 & 12 \\
\hline $\begin{array}{l}\text { Variable } 37 \text { (cols } 73 \& \\
74 \text { ) }\end{array}$ & 100 & 1 & 1 & 1 & 6 & 0 & 6 & 12 \\
\hline $\begin{array}{l}\text { Variable } 38 \text { (cols } 75 \& \\
\text { 76) }\end{array}$ & 100 & 1 & 1 & 1 & 6 & 0 & 6 & 12 \\
\hline $\begin{array}{l}\text { Variable } 39 \text { (cols } 77 \& \\
\text { 78) }\end{array}$ & 100 & 1 & 1 & 1 & 6 & 0 & 6 & 12 \\
\hline $\begin{array}{l}\text { Variable } 40 \text { (cols } 79 \& \\
80 \text { ) }\end{array}$ & 100 & 1 & 1 & 1 & 6 & 0 & 6 & 12 \\
\hline $\begin{array}{l}\text { Variable } 41 \text { (cols } 81 \& \\
82 \text { ) }\end{array}$ & 100 & 1 & 1 & 1 & 6 & 0 & 6 & 12 \\
\hline $\begin{array}{l}\text { Variable } 42 \text { (cols } 83 \& \\
84 \text { ) }\end{array}$ & 100 & 1 & 1 & 1 & 6 & 0 & 6 & 12 \\
\hline $\begin{array}{l}\text { Variable } 43 \text { (cols } 85 \& \\
86 \text { ) }\end{array}$ & 100 & 1 & 1 & 1 & 6 & 0 & 6 & 12 \\
\hline $\begin{array}{l}\text { Variable } 44 \text { (cols } 87 \& \\
88 \text { ) }\end{array}$ & 100 & 1 & 1 & 1 & 6 & 0 & 6 & 12 \\
\hline $\begin{array}{l}\text { Variable } 45 \text { (cols } 89 \& \\
90 \text { ) }\end{array}$ & 100 & 1 & 1 & 1 & 6 & 0 & 6 & 12 \\
\hline $\begin{array}{l}\text { Variable } 46 \text { (cols } 91 \& \\
92 \text { ) }\end{array}$ & 100 & 1 & 1 & 1 & 6 & 0 & 6 & 12 \\
\hline $\begin{array}{l}\text { Variable } 47 \text { (cols } 93 \& \\
94 \text { ) }\end{array}$ & 100 & 1 & 1 & 1 & 6 & 0 & 6 & 12 \\
\hline $\begin{array}{l}\text { Variable } 48 \text { (cols } 95 \& \\
96 \text { ) }\end{array}$ & 100 & 1 & 1 & 1 & 6 & 0 & 6 & 12 \\
\hline $\begin{array}{l}\text { Variable } 49 \text { (cols } 97 \& \\
98 \text { ) }\end{array}$ & 100 & 1 & 1 & 1 & 6 & 0 & 6 & 12 \\
\hline $\begin{array}{l}\text { Variable } 50 \text { (cols } 99 \& \\
100 \text { ) }\end{array}$ & 100 & 1 & 1 & 1 & 6 & 0 & 6 & 12 \\
\hline $\begin{array}{l}\text { Variable } 51 \text { (cols } 101 \text { \& } \\
102 \text { ) }\end{array}$ & 100 & 1 & 1 & 1 & 6 & 0 & 6 & 12 \\
\hline
\end{tabular}




\begin{tabular}{|c|c|c|c|c|c|c|c|c|}
\hline & \begin{tabular}{|l} 
Percent \\
Agreement
\end{tabular} & Scott's Pi & Cohen's Kappa & $\begin{array}{l}\text { Krippendorff's } \\
\text { Alpha }\end{array}$ & N Agreements & $\begin{array}{l}\mathrm{N} \\
\text { Disagreements }\end{array}$ & N Cases & N Decisions \\
\hline $\begin{array}{l}\text { Variable } 52 \text { (cols } 103 \text { \& } \\
104 \text { ) }\end{array}$ & 100 & 1 & 1 & 1 & 6 & 0 & 6 & 12 \\
\hline $\begin{array}{l}\text { Variable } 53 \text { (cols } 105 \text { \& } \\
\text { 106) }\end{array}$ & 100 & 1 & 1 & 1 & 6 & 0 & 6 & 12 \\
\hline $\begin{array}{l}\text { Variable } 54 \text { (cols } 107 \text { \& } \\
\text { 108) }\end{array}$ & 100 & 1 & 1 & 1 & 6 & 0 & 6 & 12 \\
\hline $\begin{array}{l}\text { Variable } 55 \text { (cols } 109 \text { \& } \\
110 \text { ) }\end{array}$ & 100 & 1 & 1 & 1 & 6 & 0 & 6 & 12 \\
\hline $\begin{array}{l}\text { Variable } 56 \text { (cols } 111 \text { \& } \\
112 \text { ) }\end{array}$ & 100 & 1 & 1 & 1 & 6 & 0 & 6 & 12 \\
\hline $\begin{array}{l}\text { Variable } 57 \text { (cols } 113 \text { \& } \\
114 \text { ) }\end{array}$ & 100 & 1 & 1 & 1 & 6 & 0 & 6 & 12 \\
\hline $\begin{array}{l}\text { Variable } 58 \text { (cols } 115 \text { \& } \\
116)\end{array}$ & 100 & 1 & 1 & 1 & 6 & 0 & 6 & 12 \\
\hline $\begin{array}{l}\text { Variable } 59 \text { (cols } 117 \text { \& } \\
118 \text { ) }\end{array}$ & 100 & 1 & 1 & 1 & 6 & 0 & 6 & 12 \\
\hline $\begin{array}{l}\text { Variable } 60 \text { (cols } 119 \text { \& } \\
120 \text { ) }\end{array}$ & 100 & 1 & 1 & 1 & 6 & 0 & 6 & 12 \\
\hline $\begin{array}{l}\text { Variable } 61 \text { (cols } 121 \text { \& } \\
122 \text { ) }\end{array}$ & 100 & 1 & 1 & 1 & 6 & 0 & 6 & 12 \\
\hline $\begin{array}{l}\text { Variable } 62 \text { (cols } 123 \text { \& } \\
124 \text { ) }\end{array}$ & 83.33333 & -0.09091 & $6.66 \mathrm{E}-16$ & 0 & 5 & 1 & 6 & 12 \\
\hline $\begin{array}{l}\text { Variable } 63 \text { (cols } 125 \text { \& } \\
126 \text { ) }\end{array}$ & 100 & 1 & 1 & 1 & 6 & 0 & 6 & 12 \\
\hline $\begin{array}{l}\text { Variable } 64 \text { (cols } 127 \text { \& } \\
128 \text { ) }\end{array}$ & 100 & 1 & 1 & 1 & 6 & 0 & 6 & 12 \\
\hline $\begin{array}{l}\text { Variable } 65 \text { (cols } 129 \& \\
130 \text { ) }\end{array}$ & 100 & 1 & 1 & 1 & 6 & 0 & 6 & 12 \\
\hline $\begin{array}{l}\text { Variable } 66 \text { (cols } 131 \text { \& } \\
132 \text { ) }\end{array}$ & 100 & 1 & 1 & 1 & 6 & 0 & 6 & 12 \\
\hline $\begin{array}{l}\text { Variable } 67 \text { (cols } 133 \text { \& } \\
\text { 134) }\end{array}$ & 100 & 1 & 1 & 1 & 6 & 0 & 6 & 12 \\
\hline $\begin{array}{l}\text { Variable } 68 \text { (cols } 135 \text { \& } \\
136 \text { ) }\end{array}$ & 100 & 1 & 1 & 1 & 6 & 0 & 6 & 12 \\
\hline Variable 69 (cols 137 \& & 100 & 1 & 1 & 1 & 6 & 0 & 6 & 12 \\
\hline
\end{tabular}




\begin{tabular}{|c|c|c|c|c|c|c|c|c|}
\hline & \begin{tabular}{|l|} 
Percent \\
Agreement
\end{tabular} & Scott's Pi & Cohen's Kappa & $\begin{array}{l}\text { Krippendorf's } \\
\text { Alpha }\end{array}$ & N Agreements & \begin{tabular}{|l|l|}
$\mathrm{N}$ \\
Disagreements
\end{tabular} & N Cases & N Decisions \\
\hline 138) & & & & & & & & \\
\hline $\begin{array}{l}\text { Variable } 70 \text { (cols } 139 \text { \& } \\
140 \text { ) }\end{array}$ & 100 & 1 & 1 & 1 & 6 & 0 & 6 & 12 \\
\hline $\begin{array}{l}\text { Variable } 71 \text { (cols } 141 \& \\
142 \text { ) }\end{array}$ & 100 & 1 & 1 & 1 & 6 & 0 & 6 & 12 \\
\hline $\begin{array}{l}\text { Variable } 72 \text { (cols } 143 \text { \& } \\
144 \text { ) }\end{array}$ & 100 & 1 & 1 & 1 & 6 & 0 & 6 & 12 \\
\hline $\begin{array}{l}\text { Variable } 73 \text { (cols } 145 \text { \& } \\
146 \text { ) }\end{array}$ & 100 & 1 & 1 & 1 & 6 & 0 & 6 & 12 \\
\hline $\begin{array}{l}\text { Variable } 74 \text { (cols } 147 \text { \& } \\
\text { 148) }\end{array}$ & 100 & 1 & 1 & 1 & 6 & 0 & 6 & 12 \\
\hline $\begin{array}{l}\text { Variable } 75 \text { (cols } 149 \text { \& } \\
150 \text { ) }\end{array}$ & 100 & 1 & 1 & 1 & 6 & 0 & 6 & 12 \\
\hline $\begin{array}{l}\text { Variable } 76 \text { (cols } 151 \text { \& } \\
152 \text { ) }\end{array}$ & 100 & 1 & 1 & 1 & 6 & 0 & 6 & 12 \\
\hline $\begin{array}{l}\text { Variable } 77 \text { (cols } 153 \text { \& } \\
154 \text { ) }\end{array}$ & 83.33333 & -0.09091 & $6.66 \mathrm{E}-16$ & 0 & 5 & 1 & 6 & 12 \\
\hline $\begin{array}{l}\text { Variable } 78 \text { (cols } 155 \text { \& } \\
156)\end{array}$ & 100 & 1 & 1 & 1 & 6 & 0 & 6 & 12 \\
\hline $\begin{array}{l}\text { Variable } 79 \text { (cols } 157 \text { \& } \\
\text { 158) }\end{array}$ & 100 & 1 & 1 & 1 & 6 & 0 & 6 & 12 \\
\hline $\begin{array}{l}\text { Variable } 80 \text { (cols } 159 \text { \& } \\
160 \text { ) }\end{array}$ & 100 & 1 & 1 & 1 & 6 & 0 & 6 & 12 \\
\hline $\begin{array}{l}\text { Variable } 81 \text { (cols } 161 \text { \& } \\
162 \text { ) }\end{array}$ & 83.33333 & 0.773585 & 0.777778 & 0.792453 & 5 & 1 & 6 & 12 \\
\hline $\begin{array}{l}\text { Variable } 82 \text { (cols } 163 \text { \& } \\
\text { 164) }\end{array}$ & 100 & 1 & 1 & 1 & 6 & 0 & 6 & 12 \\
\hline $\begin{array}{l}\text { Variable } 83 \text { (cols } 165 \text { \& } \\
\text { 166) }\end{array}$ & 83.33333 & -0.09091 & $6.66 \mathrm{E}-16$ & 0 & 5 & 1 & 6 & 12 \\
\hline $\begin{array}{l}\text { Variable } 84 \text { (cols } 167 \text { \& } \\
168 \text { ) }\end{array}$ & 100 & 1 & 1 & 1 & 6 & 0 & 6 & 12 \\
\hline $\begin{array}{l}\text { Variable } 85 \text { (cols } 169 \text { \& } \\
\text { 170) }\end{array}$ & 100 & 1 & 1 & 1 & 6 & 0 & 6 & 12 \\
\hline $\begin{array}{l}\text { Variable } 86 \text { (cols } 171 \text { \& } \\
172 \text { ) }\end{array}$ & 100 & 1 & 1 & 1 & 6 & 0 & 6 & 12 \\
\hline
\end{tabular}




\begin{tabular}{|c|c|c|c|c|c|c|c|c|}
\hline & \begin{tabular}{|l} 
Percent \\
Agreement
\end{tabular} & scott's Pi & Cohen's Kappa & \begin{tabular}{|l} 
Krippendorf's \\
Alpha
\end{tabular} & N Agreements & $\begin{array}{l}\mathrm{N} \\
\text { Disagreements }\end{array}$ & N Cases & N Decisions \\
\hline $\begin{array}{l}\text { Variable } 87 \text { (cols } 173 \& \\
174 \text { ) }\end{array}$ & 100 & 1 & 1 & 1 & 6 & 0 & 6 & 12 \\
\hline $\begin{array}{l}\text { Variable } 88 \text { (cols } 175 \& \\
176 \text { ) }\end{array}$ & 100 & 1 & 1 & 1 & 6 & 0 & 6 & 12 \\
\hline $\begin{array}{l}\text { Variable } 89 \text { (cols } 177 \text { \& } \\
\text { 178) }\end{array}$ & 83.33333 & -0.09091 & $6.66 \mathrm{E}-16$ & 0 & 5 & 1 & 6 & 12 \\
\hline $\begin{array}{l}\text { Variable } 90 \text { (cols } 179 \& \\
\text { 180) }\end{array}$ & 100 & 1 & 1 & 1 & 6 & 0 & 6 & 12 \\
\hline $\begin{array}{l}\text { Variable } 91 \text { (cols } 181 \& \\
\text { 182) }\end{array}$ & 100 & 1 & 1 & 1 & 6 & 0 & 6 & 12 \\
\hline $\begin{array}{l}\text { Variable } 92 \text { (cols } 183 \& \\
\text { 184) }\end{array}$ & 100 & 1 & 1 & 1 & 6 & 0 & 6 & 12 \\
\hline $\begin{array}{l}\text { Variable } 93 \text { (cols } 185 \text { \& } \\
\text { 186) }\end{array}$ & 100 & 1 & 1 & 1 & 6 & 0 & 6 & 12 \\
\hline $\begin{array}{l}\text { Variable } 94 \text { (cols } 187 \text { \& } \\
\text { 188) }\end{array}$ & 100 & 1 & 1 & 1 & 6 & 0 & 6 & 12 \\
\hline $\begin{array}{l}\text { Variable } 95 \text { (cols } 189 \& \\
\text { 190) }\end{array}$ & 100 & 1 & 1 & 1 & 6 & 0 & 6 & 12 \\
\hline $\begin{array}{l}\text { Variable } 96 \text { (cols } 191 \text { \& } \\
\text { 192) }\end{array}$ & 83.33333 & 0.428571 & 0.454545 & 0.47619 & 5 & 1 & 6 & 12 \\
\hline $\begin{array}{l}\text { Variable } 97 \text { (cols } 193 \text { \& } \\
\text { 194) }\end{array}$ & 100 & 1 & 1 & 1 & 6 & 0 & 6 & 12 \\
\hline $\begin{array}{l}\text { Variable } 98 \text { (cols } 195 \& \\
\text { 196) }\end{array}$ & 100 & 1 & 1 & 1 & 6 & 0 & 6 & 12 \\
\hline $\begin{array}{l}\text { Variable } 99 \text { (cols } 197 \text { \& } \\
\text { 198) }\end{array}$ & 100 & 1 & 1 & 1 & 6 & 0 & 6 & 12 \\
\hline $\begin{array}{l}\text { Variable } 100 \text { (cols } 199 \& \\
\text { 200) }\end{array}$ & 100 & 1 & 1 & 1 & 6 & 0 & 6 & 12 \\
\hline $\begin{array}{l}\text { Variable } 101 \text { (cols } 201 \& \\
\text { 202) }\end{array}$ & 100 & 1 & 1 & 1 & 6 & 0 & 6 & 12 \\
\hline $\begin{array}{l}\text { Variable } 102 \text { (cols } 203 \& \text { \& } \\
\text { 204) }\end{array}$ & 100 & 1 & 1 & 1 & 6 & 0 & 6 & 12 \\
\hline $\begin{array}{l}\text { Variable } 103 \text { (cols } 205 \& \\
\text { 206) }\end{array}$ & 100 & 1 & 1 & 1 & 6 & 0 & 6 & 12 \\
\hline Variable 104 (cols 207 \& & 100 & 1 & 1 & 1 & 6 & 0 & 6 & 12 \\
\hline
\end{tabular}




\begin{tabular}{|c|c|c|c|c|c|c|c|c|}
\hline & \begin{tabular}{|l|} 
Percent \\
Agreement
\end{tabular} & Scott's Pi & Cohen's Kappa & \begin{tabular}{|l}
$\mid$ Krippendorf's \\
Alpha
\end{tabular} & N Agreements & \begin{tabular}{|l|l|}
$\mathrm{N}$ \\
Disagreements
\end{tabular} & N Cases & N Decisions \\
\hline 208) & & & & & & & & \\
\hline $\begin{array}{l}\text { Variable } 105 \text { (cols } 209 \& \\
\text { 210) }\end{array}$ & 100 & 1 & 1 & 1 & 6 & 0 & 6 & 12 \\
\hline $\begin{array}{l}\text { Variable } 106 \text { (cols } 211 \text { \& } \\
\text { 212) }\end{array}$ & 100 & 1 & 1 & 1 & 6 & 0 & 6 & 12 \\
\hline $\begin{array}{l}\text { Variable } 107 \text { (cols } 213 \text { \& } \\
\text { 214) }\end{array}$ & 100 & 1 & 1 & 1 & 6 & 0 & 6 & 12 \\
\hline $\begin{array}{l}\text { Variable } 108 \text { (cols } 215 \text { \& } \\
\text { 216) }\end{array}$ & 100 & 1 & 1 & 1 & 6 & 0 & 6 & 12 \\
\hline $\begin{array}{l}\text { Variable } 109 \text { (cols } 217 \& \\
218 \text { ) }\end{array}$ & 100 & 1 & 1 & 1 & 6 & 0 & 6 & 12 \\
\hline $\begin{array}{l}\text { Variable } 110 \text { (cols } 219 \& \\
220)\end{array}$ & 100 & 1 & 1 & 1 & 6 & 0 & 6 & 12 \\
\hline $\begin{array}{l}\text { Variable } 111 \text { (cols } 221 \text { \& } \\
\text { 222) }\end{array}$ & 100 & 1 & 1 & 1 & 6 & 0 & 6 & 12 \\
\hline $\begin{array}{l}\text { Variable } 112 \text { (cols } 223 \& \\
224)\end{array}$ & 100 & 1 & 1 & 1 & 6 & 0 & 6 & 12 \\
\hline $\begin{array}{l}\text { Variable } 113 \text { (cols } 225 \& \\
226 \text { ) }\end{array}$ & 100 & 1 & 1 & 1 & 6 & 0 & 6 & 12 \\
\hline $\begin{array}{l}\text { Variable } 114 \text { (cols } 227 \& \\
\text { 228) }\end{array}$ & 100 & 1 & 1 & 1 & 6 & 0 & 6 & 12 \\
\hline $\begin{array}{l}\text { Variable } 115 \text { (cols } 229 \& \\
230 \text { ) }\end{array}$ & 100 & 1 & 1 & 1 & 6 & 0 & 6 & 12 \\
\hline $\begin{array}{l}\text { Variable } 116 \text { (cols } 231 \text { \& } \\
\text { 232) }\end{array}$ & 83.33333 & 0.657143 & 0.666667 & 7| 0.685714 & 5 & 1 & 6 & 12 \\
\hline $\begin{array}{l}\text { Variable } 117 \text { (cols } 233 \& \\
234 \text { ) }\end{array}$ & 100 & 1 & 1 & 1 & 6 & 0 & 6 & 12 \\
\hline $\begin{array}{l}\text { Variable } 118 \text { (cols } 235 \& \text { } \\
236)\end{array}$ & 100 & 1 & 1 & 1 & 6 & 0 & 6 & 12 \\
\hline $\begin{array}{l}\text { Variable } 119 \text { (cols } 237 \& \\
238 \text { ) }\end{array}$ & 83.33333 & -0.09091 & $6.66 \mathrm{E}-16$ & 0 & 5 & 1 & 6 & 12 \\
\hline $\begin{array}{l}\text { Variable } 120 \text { (cols } 239 \& \\
\text { 240) }\end{array}$ & 100 & 1 & 1 & 1 & 6 & 0 & 6 & 12 \\
\hline $\begin{array}{l}\text { Variable } 121 \text { (cols } 241 \text { \& } \\
242 \text { ) }\end{array}$ & 66.66667 & 0.586207 & 0.6 & 0.62069 & 4 & 2 & 6 & 12 \\
\hline
\end{tabular}




\begin{tabular}{|c|c|c|c|c|c|c|c|c|}
\hline & \begin{tabular}{|l} 
Percent \\
Agreement
\end{tabular} & scott's Pi & Cohen's Kappa & \begin{tabular}{|l} 
Krippendorf's \\
Alpha
\end{tabular} & N Agreements & \begin{tabular}{|l|l|}
$\mathrm{N}$ \\
Disagreements
\end{tabular} & N Cases & N Decisions \\
\hline $\begin{array}{l}\text { Variable } 122 \text { (cols } 243 \& \\
244 \text { ) }\end{array}$ & 100 & 1 & 1 & 1 & 6 & 0 & 6 & 12 \\
\hline $\begin{array}{l}\text { Variable } 123 \text { (cols } 245 \& \\
246)\end{array}$ & 100 & 1 & 1 & 1 & 6 & 0 & 6 & 12 \\
\hline $\begin{array}{l}\text { Variable } 124 \text { (cols } 247 \& \\
248 \text { ) }\end{array}$ & 100 & 1 & 1 & 1 & 6 & 0 & 6 & 12 \\
\hline $\begin{array}{l}\text { Variable } 125 \text { (cols } 249 \& \\
250 \text { ) }\end{array}$ & 100 & 1 & 1 & 1 & 6 & 0 & 6 & 12 \\
\hline $\begin{array}{l}\text { Variable } 126 \text { (cols } 251 \& \\
252 \text { ) }\end{array}$ & 83.33333 & 0.79661 & 0.8 & $3 \mid 0.813559$ & 5 & 1 & 6 & 12 \\
\hline $\begin{array}{l}\text { Variable } 127 \text { (cols } 253 \& \\
254 \text { ) }\end{array}$ & 66.66667 & 0.571429 & 0.586207 & 70.607143 & 4 & 2 & 6 & 12 \\
\hline $\begin{array}{l}\text { Variable } 128 \text { (cols } 255 \& \\
256 \text { ) }\end{array}$ & 100 & 1 & 1 & 1 & 6 & 0 & 6 & 12 \\
\hline $\begin{array}{l}\text { Variable } 129 \text { (cols } 257 \& \\
\text { 258) }\end{array}$ & 100 & 1 & 1 & 1 & 6 & 0 & 6 & 12 \\
\hline $\begin{array}{l}\text { Variable } 130 \text { (cols } 259 \& \\
260 \text { ) }\end{array}$ & 100 & 1 & 1 & 1 & 6 & 0 & 6 & 12 \\
\hline $\begin{array}{l}\text { Variable } 131 \text { (cols } 261 \& \\
262 \text { ) }\end{array}$ & 100 & 1 & 1 & 1 & 6 & 0 & 6 & 12 \\
\hline $\begin{array}{l}\text { Variable } 132 \text { (cols } 263 \& \\
264 \text { ) }\end{array}$ & 100 & 1 & 1 & 1 & 6 & 0 & 6 & 12 \\
\hline $\begin{array}{l}\text { Variable } 133 \text { (cols } 265 \& \text { } \\
\text { 266) }\end{array}$ & 100 & 1 & 1 & 1 & 6 & 0 & 6 & 12 \\
\hline $\begin{array}{l}\text { Variable } 134 \text { (cols } 267 \& \\
\text { 268) }\end{array}$ & 100 & 1 & 1 & 1 & 6 & 0 & 6 & 12 \\
\hline $\begin{array}{l}\text { Variable } 135 \text { (cols } 269 \& \\
\text { 270) }\end{array}$ & 100 & 1 & 1 & 1 & 6 & 0 & 6 & 12 \\
\hline $\begin{array}{l}\text { Variable } 136 \text { (cols } 271 \& \\
\text { 272) }\end{array}$ & 83.33333 & 0.586207 & 0.6 & 0.62069 & 5 & 1 & 6 & 12 \\
\hline $\begin{array}{l}\text { Variable } 137 \text { (cols } 273 \& \\
\text { 274) }\end{array}$ & 100 & 1 & 1 & 1 & 6 & 0 & 6 & 12 \\
\hline $\begin{array}{l}\text { Variable } 138 \text { (cols } 275 \& \\
276)\end{array}$ & 100 & 1 & 1 & 1 & 6 & 0 & 6 & 12 \\
\hline Variable 139 (cols $277 \&$ & 83.33333 & 0.428571 & 0.454545 & 0.47619 & 5 & 1 & 6 & 12 \\
\hline
\end{tabular}




\begin{tabular}{|c|c|c|c|c|c|c|c|c|}
\hline & $\begin{array}{l}\text { Percent } \\
\text { Agreement }\end{array}$ & Scott's Pi & Cohen's Kappa & $\begin{array}{l}\text { Krippendorf's } \\
\text { Alpha }\end{array}$ & N Agreements & \begin{tabular}{|l|l|}
$\mathrm{N}$ \\
Disagreements
\end{tabular} & N Cases & N Decisions \\
\hline 278) & & & & & & & & \\
\hline $\begin{array}{l}\text { Variable } 140 \text { (cols } 279 \& \\
280 \text { ) }\end{array}$ & 100 & 1 & 1 & 1 & 6 & 0 & 6 & 12 \\
\hline $\begin{array}{l}\text { Variable } 141 \text { (cols } 281 \text { \& } \\
\text { 282) }\end{array}$ & 66.66667 & 0.454545 & 0.5 & 0.5 & 4 & 2 & 6 & 12 \\
\hline $\begin{array}{l}\text { Variable } 142 \text { (cols } 283 \& \\
\text { 284) }\end{array}$ & 100 & 1 & 1 & 1 & 6 & 0 & 6 & 12 \\
\hline $\begin{array}{l}\text { Variable } 143 \text { (cols } 285 \& \\
\text { 286) }\end{array}$ & 100 & 1 & 1 & 1 & 6 & 0 & 6 & 12 \\
\hline $\begin{array}{l}\text { Variable } 144 \text { (cols } 287 \& \\
288 \text { ) }\end{array}$ & 100 & 1 & 1 & 1 & 6 & 0 & 6 & 12 \\
\hline $\begin{array}{l}\text { Variable } 145 \text { (cols } 289 \& \\
\text { 290) }\end{array}$ & 100 & 1 & 1 & 1 & 6 & 0 & 6 & 12 \\
\hline $\begin{array}{l}\text { Variable } 146 \text { (cols } 291 \text { \& } \\
\text { 292) }\end{array}$ & 83.33333 & -0.09091 & $6.66 \mathrm{E}-16$ & 0 & 5 & 1 & 6 & 12 \\
\hline $\begin{array}{l}\text { Variable } 147 \text { (cols } 293 \& \\
294 \text { \&) }\end{array}$ & 100 & 1 & 1 & 1 & 6 & 0 & 6 & 12 \\
\hline $\begin{array}{l}\text { Variable } 148 \text { (cols } 295 \& \\
\text { 296) }\end{array}$ & 100 & 1 & 1 & 1 & 6 & 0 & 6 & 12 \\
\hline $\begin{array}{l}\text { Variable } 149 \text { (cols } 297 \& \\
\text { 298) }\end{array}$ & 100 & 1 & 1 & 1 & 6 & 0 & 6 & 12 \\
\hline $\begin{array}{l}\text { Variable } 150 \text { (cols } 299 \text { \& } \\
300 \text { ) }\end{array}$ & 83.33333 & -0.09091 & $6.66 \mathrm{E}-16$ & 0 & 5 & 1 & 6 & 12 \\
\hline $\begin{array}{l}\text { Variable } 151 \text { (cols } 301 \& \\
302 \text { ) }\end{array}$ & 100 & 1 & 1 & 1 & 6 & 0 & 6 & 12 \\
\hline $\begin{array}{l}\text { Variable } 152 \text { (cols } 303 \text { \& } \\
304 \text { ) }\end{array}$ & 100 & 1 & 1 & 1 & 6 & 0 & 6 & 12 \\
\hline $\begin{array}{l}\text { Variable } 153 \text { (cols } 305 \text { \& } \\
306)\end{array}$ & 83.33333 & 0.657143 & 0.666667 & 0.685714 & 5 & 1 & 6 & 12 \\
\hline $\begin{array}{l}\text { Variable } 154 \text { (cols } 307 \text { \& } \\
308 \text { ) }\end{array}$ & 100 & 1 & 1 & 1 & 6 & 0 & 6 & 12 \\
\hline $\begin{array}{l}\text { Variable } 155 \text { (cols } 309 \text { \& } \\
\text { 310) }\end{array}$ & 100 & 1 & 1 & 1 & 6 & 0 & 6 & 12 \\
\hline $\begin{array}{l}\text { Variable } 156 \text { (cols } 311 \text { \& } \\
312 \text { ) }\end{array}$ & 100 & 1 & 1 & 1 & 6 & 0 & 6 & 12 \\
\hline
\end{tabular}




\begin{tabular}{|c|c|c|c|c|c|c|c|c|}
\hline & \begin{tabular}{|l} 
Percent \\
Agreement
\end{tabular} & scott's Pi & Cohen's Kappa & $\begin{array}{l}\text { Krippendorff's } \\
\text { Alpha }\end{array}$ & N Agreements & \begin{tabular}{|l|l|}
$\mathrm{N}$ \\
Disagreements
\end{tabular} & N Cases & N Decisions \\
\hline $\begin{array}{l}\text { Variable } 157 \text { (cols } 313 \text { \& } \\
314 \text { ) }\end{array}$ & 100 & 1 & 1 & 1 & 6 & 0 & 6 & 12 \\
\hline $\begin{array}{l}\text { Variable } 158 \text { (cols } 315 \& \\
316)\end{array}$ & 100 & 1 & 1 & 1 & 6 & 0 & 6 & 12 \\
\hline $\begin{array}{l}\text { Variable } 159 \text { (cols } 317 \text { \& } \\
318)\end{array}$ & 100 & 1 & 1 & 1 & 6 & 0 & 6 & 12 \\
\hline $\begin{array}{l}\text { Variable } 160 \text { (cols } 319 \& \\
320)\end{array}$ & 100 & 1 & 1 & 1 & 6 & 0 & 6 & 12 \\
\hline $\begin{array}{l}\text { Variable } 161 \text { (cols } 321 \& \\
322 \text { ) }\end{array}$ & 100 & 1 & 1 & 1 & 6 & 0 & 6 & 12 \\
\hline $\begin{array}{l}\text { Variable } 162 \text { (cols } 323 \text { \& } \\
324 \text { ) }\end{array}$ & 100 & 1 & 1 & 1 & 6 & 0 & 6 & 12 \\
\hline $\begin{array}{l}\text { Variable } 163 \text { (cols } 325 \text { \& } \\
326 \text { ) }\end{array}$ & 100 & 1 & 1 & 1 & 6 & 0 & 6 & 12 \\
\hline $\begin{array}{l}\text { Variable } 164 \text { (cols } 327 \& \\
328)\end{array}$ & 100 & 1 & 1 & 1 & 6 & 0 & 6 & 12 \\
\hline $\begin{array}{l}\text { Variable } 165 \text { (cols } 329 \& \\
330)\end{array}$ & 100 & 1 & 1 & 1 & 6 & 0 & 6 & 12 \\
\hline $\begin{array}{l}\text { Variable } 166 \text { (cols } 331 \& \\
332 \text { ) }\end{array}$ & 83.33333 & 0.744681 & 0.75 & 0.765957 & 5 & 1 & 6 & 12 \\
\hline $\begin{array}{l}\text { Variable } 167 \text { (cols } 333 \& \\
334 \text { \&) }\end{array}$ & 66.66667 & 0.5 & 0.5 & 0.541667 & 4 & 2 & 6 & 12 \\
\hline $\begin{array}{l}\text { Variable } 168 \text { (cols } 335 \& \text { } \\
336)\end{array}$ & 100 & 1 & 1 & 1 & 6 & 0 & 6 & 12 \\
\hline $\begin{array}{l}\text { Variable } 169 \text { (cols } 337 \text { \& } \\
\text { 338) }\end{array}$ & 100 & 1 & 1 & 1 & 6 & 0 & 6 & 12 \\
\hline $\begin{array}{l}\text { Variable } 170 \text { (cols } 339 \& \\
340 \text { ) }\end{array}$ & 100 & 1 & 1 & 1 & 6 & 0 & 6 & 12 \\
\hline $\begin{array}{l}\text { Variable } 171 \text { (cols } 341 \text { \& } \\
342 \text { ) }\end{array}$ & 83.33333 & 0.675676 & 0.684211 & 0.702703 & 5 & 1 & 6 & 12 \\
\hline $\begin{array}{l}\text { Variable } 172 \text { (cols } 343 \text { \& } \\
344 \text { ) }\end{array}$ & 83.33333 & 0.707317 & 0.714286 & 0.731707 & 5 & 1 & 6 & 12 \\
\hline $\begin{array}{l}\text { Variable } 173 \text { (cols } 345 \& \text { } \\
346 \text { ) }\end{array}$ & 100 & 1 & 1 & 1 & 6 & 0 & 6 & 12 \\
\hline Variable 174 (cols 347 \& & 100 & 1 & 1 & 1 & 6 & 0 & 6 & 12 \\
\hline
\end{tabular}




\begin{tabular}{|c|c|c|c|c|c|c|c|c|}
\hline & \begin{tabular}{|l|} 
Percent \\
Agreement
\end{tabular} & Scott's pi & Cohen's Kappa & $\begin{array}{l}\text { Krippendorf's } \\
\text { Alpha }\end{array}$ & N Agreements & \begin{tabular}{|l|l|}
$\mathrm{N}$ \\
Disagreements
\end{tabular} & N Cases & N Decisions \\
\hline 348) & & & & & & & & \\
\hline $\begin{array}{l}\text { Variable } 175 \text { (cols } 349 \& \\
350)\end{array}$ & 100 & 1 & 1 & 1 & 6 & 0 & 6 & 12 \\
\hline $\begin{array}{l}\text { Variable } 176 \text { (cols } 351 \text { \& } \\
352 \text { ) }\end{array}$ & 100 & 1 & 1 & 1 & 6 & 0 & 6 & 12 \\
\hline $\begin{array}{l}\text { Variable } 177 \text { (cols } 353 \text { \& } \\
354 \text { ) }\end{array}$ & 100 & 1 & 1 & 1 & 6 & 0 & 6 & 12 \\
\hline $\begin{array}{l}\text { Variable } 178 \text { (cols } 355 \text { \& } \\
356 \text { ) }\end{array}$ & 100 & 1 & 1 & 1 & 6 & 0 & 6 & 12 \\
\hline $\begin{array}{l}\text { Variable } 179 \text { (cols } 357 \& \\
358 \text { ) }\end{array}$ & 100 & 1 & 1 & 1 & 6 & 0 & 6 & 12 \\
\hline $\begin{array}{l}\text { Variable } 180 \text { (cols } 359 \text { \& } \\
360 \text { ) }\end{array}$ & 100 & 1 & 1 & 1 & 6 & 0 & 6 & 12 \\
\hline $\begin{array}{l}\text { Variable } 181 \text { (cols } 361 \& \\
362 \text { ) }\end{array}$ & 100 & 1 & 1 & 1 & 6 & 0 & 6 & 12 \\
\hline $\begin{array}{l}\text { Variable } 182 \text { (cols } 363 \& \\
364 \text { ) }\end{array}$ & 100 & 1 & 1 & 1 & 6 & 0 & 6 & 12 \\
\hline $\begin{array}{l}\text { Variable } 183 \text { (cols } 365 \& \\
366 \text { ) }\end{array}$ & 100 & 1 & 1 & 1 & 6 & 0 & 6 & 12 \\
\hline $\begin{array}{l}\text { Variable } 184 \text { (cols } 367 \& \\
368 \text { ) }\end{array}$ & 100 & 1 & 1 & 1 & 6 & 0 & 6 & 12 \\
\hline $\begin{array}{l}\text { Variable } 185 \text { (cols } 369 \& \\
370 \text { ) }\end{array}$ & 100 & 1 & 1 & 1 & 6 & 0 & 6 & 12 \\
\hline $\begin{array}{l}\text { Variable } 186 \text { (cols } 371 \& \\
372 \text { ) }\end{array}$ & 83.33333 & 0.79661 & 0.8 & 0.813559 & 5 & 1 & 6 & 12 \\
\hline $\begin{array}{l}\text { Variable } 187 \text { (cols } 373 \& \\
374 \text { ) }\end{array}$ & 100 & 1 & 1 & 1 & 6 & 0 & 6 & 12 \\
\hline $\begin{array}{l}\text { Variable } 188 \text { (cols } 375 \& \text { \& } \\
376)\end{array}$ & 100 & 1 & 1 & 1 & 6 & 0 & 6 & 12 \\
\hline $\begin{array}{l}\text { Variable } 189 \text { (cols } 377 \text { \& } \\
378 \text { ) }\end{array}$ & 100 & 1 & 1 & 1 & 6 & 0 & 6 & 12 \\
\hline $\begin{array}{l}\text { Variable } 190 \text { (cols } 379 \text { \& } \\
380 \text { ) }\end{array}$ & 100 & 1 & 1 & 1 & 6 & 0 & 6 & 12 \\
\hline $\begin{array}{l}\text { Variable } 191 \text { (cols } 381 \text { \& } \\
\text { 382) }\end{array}$ & 100 & 1 & 1 & 1 & 6 & 0 & 6 & 12 \\
\hline
\end{tabular}




\begin{tabular}{|c|c|c|c|c|c|c|c|c|}
\hline & \begin{tabular}{|l} 
Percent \\
Agreement
\end{tabular} & scott's Pi & Cohen's Kappa & \begin{tabular}{|l} 
Krippendorf's \\
Alpha
\end{tabular} & N Agreements & \begin{tabular}{|l|l|}
$\mathrm{N}$ \\
Disagreements
\end{tabular} & N Cases & N Decisions \\
\hline $\begin{array}{l}\text { Variable } 192 \text { (cols } 383 \text { \& } \\
\text { 384) }\end{array}$ & 100 & 1 & 1 & 1 & 6 & 0 & 6 & 12 \\
\hline $\begin{array}{l}\text { Variable } 193 \text { (cols } 385 \text { \& } \\
386 \text { ) }\end{array}$ & 100 & 1 & 1 & 1 & 6 & 0 & 6 & 12 \\
\hline $\begin{array}{l}\text { Variable } 194 \text { (cols } 387 \& \\
388 \text { ) }\end{array}$ & 100 & 1 & 1 & 1 & 6 & 0 & 6 & 12 \\
\hline $\begin{array}{l}\text { Variable } 195 \text { (cols } 389 \text { \& } \\
390)\end{array}$ & 100 & 1 & 1 & 1 & 6 & 0 & 6 & 12 \\
\hline $\begin{array}{l}\text { Variable } 196 \text { (cols } 391 \text { \& } \\
\text { 392) }\end{array}$ & 100 & 1 & 1 & 1 & 6 & 0 & 6 & 12 \\
\hline $\begin{array}{l}\text { Variable } 197 \text { (cols } 393 \text { \& } \\
\text { 394) }\end{array}$ & 83.33333 & -0.09091 & $6.66 \mathrm{E}-16$ & 0 & 5 & 1 & 6 & 12 \\
\hline $\begin{array}{l}\text { Variable } 198 \text { (cols } 395 \& \\
\text { 396) }\end{array}$ & 83.33333 & 0.428571 & 0.454545 & 0.47619 & 5 & 1 & 6 & 12 \\
\hline $\begin{array}{l}\text { Variable } 199 \text { (cols } 397 \text { \& } \\
\text { 398) }\end{array}$ & 100 & 1 & 1 & 1 & 6 & 0 & 6 & 12 \\
\hline $\begin{array}{l}\text { Variable } 200 \text { (cols } 399 \& \\
400 \text { ) }\end{array}$ & 100 & 1 & 1 & 1 & 6 & 0 & 6 & 12 \\
\hline $\begin{array}{l}\text { Variable } 201 \text { (cols } 401 \text { \& } \\
402 \text { ) }\end{array}$ & 100 & 1 & 1 & 1 & 6 & 0 & 6 & 12 \\
\hline $\begin{array}{l}\text { Variable } 202 \text { (cols } 403 \text { \& } \\
404 \text { ) }\end{array}$ & 100 & 1 & 1 & 1 & 6 & 0 & 6 & 12 \\
\hline $\begin{array}{l}\text { Variable } 203 \text { (cols } 405 \& \\
406 \text { ) }\end{array}$ & 100 & 1 & 1 & 1 & 6 & 0 & 6 & 12 \\
\hline $\begin{array}{l}\text { Variable } 204 \text { (cols } 407 \text { \& } \\
\text { 408) }\end{array}$ & 100 & 1 & 1 & 1 & 6 & 0 & 6 & 12 \\
\hline $\begin{array}{l}\text { Variable } 205 \text { (cols } 409 \& \\
410 \text { ) }\end{array}$ & 100 & 1 & 1 & 1 & 6 & 0 & 6 & 12 \\
\hline $\begin{array}{l}\text { Variable } 206 \text { (cols } 411 \text { \& } \\
412 \text { ) }\end{array}$ & 100 & 1 & 1 & 1 & 6 & 0 & 6 & 12 \\
\hline $\begin{array}{l}\text { Variable } 207 \text { (cols } 413 \text { \& } \\
414 \text { ) }\end{array}$ & 100 & 1 & 1 & 1 & 6 & 0 & 6 & 12 \\
\hline $\begin{array}{l}\text { Variable } 208 \text { (cols } 415 \text { \& } \\
416 \text { ) }\end{array}$ & 100 & 1 & 1 & 1 & 6 & 0 & 6 & 12 \\
\hline Variable 209 (cols 417 \& & 100 & 1 & 1 & 1 & 6 & 0 & 6 & 12 \\
\hline
\end{tabular}




\begin{tabular}{|c|c|c|c|c|c|c|c|c|}
\hline & $\begin{array}{l}\text { Percent } \\
\text { Agreement }\end{array}$ & Scott's Pi & Cohen's Kappa & $\begin{array}{l}\text { Krippendorff's } \\
\text { Alpha }\end{array}$ & N Agreements & \begin{tabular}{|l|l|}
$\mathrm{N}$ \\
Disagreements
\end{tabular} & N Cases & N Decisions \\
\hline 418) & & & & & & & & \\
\hline $\begin{array}{l}\text { Variable } 210 \text { (cols } 419 \& \\
420)\end{array}$ & 100 & 1 & 1 & 1 & 6 & 0 & 6 & 12 \\
\hline $\begin{array}{l}\text { Variable } 211 \text { (cols } 421 \text { \& } \\
422 \text { ) }\end{array}$ & 83.33333 & -0.09091 & $6.66 \mathrm{E}-16$ & 0 & 5 & 1 & 6 & 12 \\
\hline $\begin{array}{l}\text { Variable } 212 \text { (cols } 423 \& \\
424 \text { ) }\end{array}$ & 100 & 1 & 1 & 1 & 6 & 0 & 6 & 12 \\
\hline $\begin{array}{l}\text { Variable } 213 \text { (cols } 425 \text { \& } \\
426 \text { ) }\end{array}$ & 100 & 1 & 1 & 1 & 6 & 0 & 6 & 12 \\
\hline $\begin{array}{l}\text { Variable } 214 \text { (cols } 427 \& \\
428 \text { ) }\end{array}$ & 100 & 1 & 1 & 1 & 6 & 0 & 6 & 12 \\
\hline $\begin{array}{l}\text { Variable } 215 \text { (cols } 429 \& \\
430 \text { ) }\end{array}$ & 100 & 1 & 1 & 1 & 6 & 0 & 6 & 12 \\
\hline $\begin{array}{l}\text { Variable } 216 \text { (cols } 431 \text { \& } \\
432 \text { ) }\end{array}$ & 100 & 1 & 1 & 1 & 6 & 0 & 6 & 12 \\
\hline $\begin{array}{l}\text { Variable } 217 \text { (cols } 433 \& \\
434)\end{array}$ & 100 & 1 & 1 & 1 & 6 & 0 & 6 & 12 \\
\hline $\begin{array}{l}\text { Variable } 218 \text { (cols } 435 \& \\
436 \text { ) }\end{array}$ & 100 & 1 & 1 & 1 & 6 & 0 & 6 & 12 \\
\hline $\begin{array}{l}\text { Variable } 219 \text { (cols } 437 \text { \& } \\
\text { 438) }\end{array}$ & 100 & 1 & 1 & 1 & 6 & 0 & 6 & 12 \\
\hline $\begin{array}{l}\text { Variable } 220 \text { (cols } 439 \& \\
440 \text { ) }\end{array}$ & 100 & 1 & 1 & 1 & 6 & 0 & 6 & 12 \\
\hline $\begin{array}{l}\text { Variable } 221 \text { (cols } 441 \text { \& } \\
442 \text { ) }\end{array}$ & 100 & 1 & 1 & 1 & 6 & 0 & 6 & 12 \\
\hline $\begin{array}{l}\text { Variable } 222 \text { (cols } 443 \text { \& } \\
444 \text { ) }\end{array}$ & 100 & 1 & 1 & 1 & 6 & 0 & 6 & 12 \\
\hline $\begin{array}{l}\text { Variable } 223 \text { (cols } 445 \& \text { } \\
446)\end{array}$ & 100 & 1 & 1 & 1 & 6 & 0 & 6 & 12 \\
\hline $\begin{array}{l}\text { Variable } 224 \text { (cols } 447 \text { \& } \\
448 \text { ) }\end{array}$ & 100 & 1 & 1 & 1 & 6 & 0 & 6 & 12 \\
\hline $\begin{array}{l}\text { Variable } 225 \text { (cols } 449 \& \\
450)\end{array}$ & 100 & 1 & 1 & 1 & 6 & 0 & 6 & 12 \\
\hline $\begin{array}{l}\text { Variable } 226 \text { (cols } 451 \& \\
452 \text { ) }\end{array}$ & 100 & 1 & 1 & 1 & 6 & 0 & 6 & 12 \\
\hline
\end{tabular}




\begin{tabular}{|c|c|c|c|c|c|c|c|c|}
\hline & \begin{tabular}{|l} 
Percent \\
Agreement
\end{tabular} & scott's Pi & Cohen's Kappa & $\begin{array}{l}\text { Krippendorff's } \\
\text { Alpha }\end{array}$ & N Agreements & \begin{tabular}{|l|l|}
$\mathrm{N}$ \\
Disagreements
\end{tabular} & N Cases & N Decisions \\
\hline $\begin{array}{l}\text { Variable } 227 \text { (cols } 453 \text { \& } \\
454 \text { ) }\end{array}$ & 100 & 1 & 1 & 1 & 6 & 0 & 6 & 12 \\
\hline $\begin{array}{l}\text { Variable } 228 \text { (cols } 455 \& \\
456)\end{array}$ & 100 & 1 & 1 & 1 & 6 & 0 & 6 & 12 \\
\hline $\begin{array}{l}\text { Variable } 229 \text { (cols } 457 \& \\
458)\end{array}$ & 100 & 1 & 1 & 1 & 6 & 0 & 6 & 12 \\
\hline $\begin{array}{l}\text { Variable } 230 \text { (cols } 459 \& \\
460 \text { ) }\end{array}$ & 100 & 1 & 1 & 1 & 6 & 0 & 6 & 12 \\
\hline $\begin{array}{l}\text { Variable } 231 \text { (cols } 461 \& \\
462 \text { ) }\end{array}$ & 100 & 1 & 1 & 1 & 6 & 0 & 6 & 12 \\
\hline $\begin{array}{l}\text { Variable } 232 \text { (cols } 463 \& \\
464 \text { ) }\end{array}$ & 100 & 1 & 1 & 1 & 6 & 0 & 6 & 12 \\
\hline $\begin{array}{l}\text { Variable } 233 \text { (cols } 465 \& \\
466 \text { ) }\end{array}$ & 100 & 1 & 1 & 1 & 6 & 0 & 6 & 12 \\
\hline $\begin{array}{l}\text { Variable } 234 \text { (cols } 467 \text { \& } \\
\text { 468) }\end{array}$ & 100 & 1 & 1 & 1 & 6 & 0 & 6 & 12 \\
\hline $\begin{array}{l}\text { Variable } 235 \text { (cols } 469 \& \\
470)\end{array}$ & 100 & 1 & 1 & 1 & 6 & 0 & 6 & 12 \\
\hline $\begin{array}{l}\text { Variable } 236 \text { (cols } 471 \& \\
472 \text { ) }\end{array}$ & 83.33333 & 0.803279 & 0.806452 & 0.819672 & 5 & 1 & 6 & 12 \\
\hline $\begin{array}{l}\text { Variable } 237 \text { (cols } 473 \text { \& } \\
474 \text { ) }\end{array}$ & 83.33333 & 0.781818 & 0.785714 & 0.8 & 5 & 1 & 6 & 12 \\
\hline $\begin{array}{l}\text { Variable } 238 \text { (cols } 475 \& \\
476 \text { ) }\end{array}$ & 83.33333 & 0.428571 & 0.454545 & 0.47619 & 5 & 1 & 6 & 12 \\
\hline $\begin{array}{l}\text { Variable } 239 \text { (cols } 477 \text { \& } \\
478 \text { ) }\end{array}$ & 83.33333 & 0.755102 & 0.76 & 0.77551 & 5 & 1 & 6 & 12 \\
\hline $\begin{array}{l}\text { Variable } 240 \text { (cols } 479 \& \\
480 \text { ) }\end{array}$ & 83.33333 & -0.09091 & $6.66 \mathrm{E}-16$ & 0 & 5 & 1 & 6 & 12 \\
\hline $\begin{array}{l}\text { Variable } 241 \text { (cols } 481 \& \\
482 \text { ) }\end{array}$ & 100 & 1 & 1 & 1 & 6 & 0 & 6 & 12 \\
\hline $\begin{array}{l}\text { Variable } 242 \text { (cols } 483 \text { \& } \\
484 \text { ) }\end{array}$ & 100 & 1 & 1 & 1 & 6 & 0 & 6 & 12 \\
\hline $\begin{array}{l}\text { Variable } 243 \text { (cols } 485 \& \\
486 \text { ) }\end{array}$ & 100 & 1 & 1 & 1 & 6 & 0 & 6 & 12 \\
\hline Variable 244 (cols 487 \& & 100 & 1 & 1 & 1 & 6 & 0 & 6 & 12 \\
\hline
\end{tabular}




\begin{tabular}{|c|c|c|c|c|c|c|c|c|}
\hline & \begin{tabular}{|l|} 
Percent \\
Agreement
\end{tabular} & Scott's Pi & Cohen's Kappa & $\begin{array}{l}\text { Krippendorff's } \\
\text { Alpha }\end{array}$ & N Agreements & \begin{tabular}{|l|l|}
$\mathrm{N}$ \\
Disagreements
\end{tabular} & N Cases & N Decisions \\
\hline 488) & & & & & & & & \\
\hline $\begin{array}{l}\text { Variable } 245 \text { (cols } 489 \& \\
490)\end{array}$ & 100 & 1 & 1 & 1 & 6 & 0 & 6 & 12 \\
\hline $\begin{array}{l}\text { Variable } 246 \text { (cols } 491 \text { \& } \\
492 \text { ) }\end{array}$ & 100 & 1 & 1 & 1 & 6 & 0 & 6 & 12 \\
\hline $\begin{array}{l}\text { Variable } 247 \text { (cols } 493 \& \\
494 \text { ) }\end{array}$ & 100 & 1 & 1 & 1 & 6 & 0 & 6 & 12 \\
\hline $\begin{array}{l}\text { Variable } 248 \text { (cols } 495 \text { \& } \\
496 \text { ) }\end{array}$ & 83.33333 & 0.586207 & 0.6 & 0.62069 & 5 & 1 & 6 & 12 \\
\hline $\begin{array}{l}\text { Variable } 249 \text { (cols } 497 \& \\
498 \text { ) }\end{array}$ & 100 & 1 & 1 & 1 & 6 & 0 & 6 & 12 \\
\hline $\begin{array}{l}\text { Variable } 250 \text { (cols } 499 \& \\
500)\end{array}$ & 100 & 1 & 1 & 1 & 6 & 0 & 6 & 12 \\
\hline $\begin{array}{l}\text { Variable } 251 \text { (cols } 501 \& \text { \& } \\
502 \text { ) }\end{array}$ & 83.33333 & 0.428571 & 0.454545 & 0.47619 & 5 & 1 & 6 & 12 \\
\hline $\begin{array}{l}\text { Variable } 252 \text { (cols } 503 \& \\
504)\end{array}$ & 100 & 1 & 1 & 1 & 6 & 0 & 6 & 12 \\
\hline $\begin{array}{l}\text { Variable } 253 \text { (cols } 505 \& \\
506 \text { ) }\end{array}$ & 100 & 1 & 1 & 1 & 6 & 0 & 6 & 12 \\
\hline $\begin{array}{l}\text { Variable } 254 \text { (cols } 507 \text { \& } \\
508 \text { ) }\end{array}$ & 100 & 1 & 1 & 1 & 6 & 0 & 6 & 12 \\
\hline $\begin{array}{l}\text { Variable } 255 \text { (cols } 509 \& \\
510 \text { ) }\end{array}$ & 100 & 1 & 1 & 1 & 6 & 0 & 6 & 12 \\
\hline $\begin{array}{l}\text { Variable } 256 \text { (cols } 511 \text { \& } \\
512 \text { ) }\end{array}$ & 100 & 1 & 1 & 1 & 6 & 0 & 6 & 12 \\
\hline $\begin{array}{l}\text { Variable } 257 \text { (cols } 513 \text { \& } \\
514 \text { ) }\end{array}$ & 83.33333 & -0.09091 & $6.66 \mathrm{E}-16$ & 0 & 5 & 1 & 6 & 12 \\
\hline $\begin{array}{l}\text { Variable } 258 \text { (cols } 515 \text { \& } \\
516 \text { ) }\end{array}$ & 100 & 1 & 1 & 1 & 6 & 0 & 6 & 12 \\
\hline $\begin{array}{l}\text { Variable } 259 \text { (cols } 517 \text { \& } \\
518 \text { ) }\end{array}$ & 100 & 1 & 1 & 1 & 6 & 0 & 6 & 12 \\
\hline $\begin{array}{l}\text { Variable } 260 \text { (cols } 519 \& \\
520 \text { ) }\end{array}$ & 100 & 1 & 1 & 1 & 6 & 0 & 6 & 12 \\
\hline $\begin{array}{l}\text { Variable } 261 \text { (cols } 521 \text { \& } \\
522 \text { ) }\end{array}$ & 83.33333 & 0.428571 & 0.454545 & 0.47619 & 5 & 1 & 6 & 12 \\
\hline
\end{tabular}




\begin{tabular}{|c|c|c|c|c|c|c|c|c|}
\hline & \begin{tabular}{|l|} 
Percent \\
Agreement
\end{tabular} & Scott's Pi & Cohen's Kappa & $\begin{array}{l}\text { Krippendorff's } \\
\text { Alpha }\end{array}$ & N Agreements & \begin{tabular}{|l|l}
$\mathrm{N}$ \\
Disagreements
\end{tabular} & N Cases & N Decisions \\
\hline $\begin{array}{l}\text { Variable } 262 \text { (cols } 523 \text { \& } \\
524 \text { ) }\end{array}$ & 100 & 1 & 1 & 1 & 6 & 0 & 6 & 12 \\
\hline $\begin{array}{l}\text { Variable } 263 \text { (cols } 525 \& \\
526 \text { ) }\end{array}$ & 100 & 1 & 1 & 1 & 6 & 0 & 6 & 12 \\
\hline $\begin{array}{l}\text { Variable } 264 \text { (cols } 527 \& \\
528 \text { ) }\end{array}$ & 100 & 1 & 1 & 1 & 6 & 0 & 6 & 12 \\
\hline $\begin{array}{l}\text { Variable } 265 \text { (cols } 529 \& \\
530 \text { ) }\end{array}$ & 100 & 1 & 1 & 1 & 6 & 0 & 6 & 12 \\
\hline $\begin{array}{l}\text { Variable } 266 \text { (cols } 531 \& \\
532 \text { ) }\end{array}$ & 100 & 1 & 1 & 1 & 6 & 0 & 6 & 12 \\
\hline $\begin{array}{l}\text { Variable } 267 \text { (cols } 533 \& \\
534 \text { ) }\end{array}$ & 100 & 1 & 1 & 1 & 6 & 0 & 6 & 12 \\
\hline $\begin{array}{l}\text { Variable } 268 \text { (cols } 535 \& \\
536 \text { ) }\end{array}$ & 100 & 1 & 1 & 1 & 6 & 0 & 6 & 12 \\
\hline $\begin{array}{l}\text { Variable } 269 \text { (cols } 537 \text { \& } \\
538 \text { ) }\end{array}$ & 100 & 1 & 1 & 1 & 6 & 0 & 6 & 12 \\
\hline $\begin{array}{l}\text { Variable } 270 \text { (cols } 539 \& \\
540 \text { ) }\end{array}$ & 100 & 1 & 1 & 1 & 6 & 0 & 6 & 12 \\
\hline $\begin{array}{l}\text { Variable } 271 \text { (cols } 541 \& \\
542 \text { ) }\end{array}$ & 100 & 1 & 1 & 1 & 6 & 0 & 6 & 12 \\
\hline $\begin{array}{l}\text { Variable } 272 \text { (cols } 543 \text { \& } \\
544 \text { ) }\end{array}$ & 100 & 1 & 1 & 1 & 6 & 0 & 6 & 12 \\
\hline $\begin{array}{l}\text { Variable } 273 \text { (cols } 545 \& \\
546 \text { ) }\end{array}$ & 100 & 1 & 1 & 1 & 6 & 0 & 6 & 12 \\
\hline $\begin{array}{l}\text { Variable } 274 \text { (cols } 547 \text { \& } \\
548 \text { ) }\end{array}$ & 100 & 1 & 1 & 1 & 6 & 0 & 6 & 12 \\
\hline $\begin{array}{l}\text { Variable } 275 \text { (cols } 549 \& \\
550 \text { ) }\end{array}$ & 100 & 1 & 1 & 1 & 6 & 0 & 6 & 12 \\
\hline $\begin{array}{l}\text { Variable } 276 \text { (cols } 551 \& \\
552 \text { ) }\end{array}$ & 100 & 1 & 1 & 1 & 6 & 0 & 6 & 12 \\
\hline $\begin{array}{l}\text { Variable } 277 \text { (cols } 553 \text { \& } \\
554 \text { ) }\end{array}$ & 100 & 1 & 1 & 1 & 6 & 0 & 6 & 12 \\
\hline $\begin{array}{l}\text { Variable } 278 \text { (cols } 555 \& \\
556 \text { ) }\end{array}$ & 100 & 1 & 1 & 1 & 6 & 0 & 6 & 12 \\
\hline Variable 279 (cols 557 \& & 100 & 1 & 1 & 1 & 6 & 0 & 6 & 12 \\
\hline
\end{tabular}




\begin{tabular}{|c|c|c|c|c|c|c|c|c|}
\hline & \begin{tabular}{|l|} 
Percent \\
Agreement
\end{tabular} & Scott's Pi & Cohen's Kappa & \begin{tabular}{|l|} 
Krippendorff's \\
Alpha
\end{tabular} & N Agreements & \begin{tabular}{|l|l|} 
N \\
Disagreements
\end{tabular} & N Cases & N Decisions \\
\hline 558) & & & & & & & & \\
\hline $\begin{array}{l}\text { Variable } 280 \text { (cols } 559 \& \\
560 \text { ) }\end{array}$ & 100 & 1 & 1 & 1 & 6 & 0 & 6 & 12 \\
\hline $\begin{array}{l}\text { Variable } 281 \text { (cols } 561 \& \\
562 \text { ) }\end{array}$ & 100 & 1 & 1 & 1 & 6 & 0 & 6 & 12 \\
\hline $\begin{array}{l}\text { Variable } 282 \text { (cols } 563 \& \\
564 \text { ) }\end{array}$ & 100 & 1 & 1 & 1 & 6 & 0 & 6 & 12 \\
\hline $\begin{array}{l}\text { Variable } 283 \text { (cols } 565 \& \\
566 \text { ) }\end{array}$ & 100 & 1 & 1 & 1 & 6 & 0 & 6 & 12 \\
\hline $\begin{array}{l}\text { Variable } 284 \text { (cols } 567 \& \\
568 \text { ) }\end{array}$ & 100 & 1 & 1 & 1 & 6 & 0 & 6 & 12 \\
\hline $\begin{array}{l}\text { Variable } 285 \text { (cols } 569 \& \\
570 \text { ) }\end{array}$ & 100 & 1 & 1 & 1 & 6 & 0 & 6 & 12 \\
\hline $\begin{array}{l}\text { Variable } 286 \text { (cols } 571 \& \\
572 \text { ) }\end{array}$ & 100 & 1 & 1 & 1 & 6 & 0 & 6 & 12 \\
\hline $\begin{array}{l}\text { Variable } 287 \text { (cols } 573 \& \\
574 \text { ) }\end{array}$ & 100 & 1 & 1 & 1 & 6 & 0 & 6 & 12 \\
\hline $\begin{array}{l}\text { Variable } 288 \text { (cols } 575 \& \\
576 \text { ) }\end{array}$ & 100 & 1 & 1 & 1 & 6 & 0 & 6 & 12 \\
\hline $\begin{array}{l}\text { Variable } 289 \text { (cols } 577 \& \\
578 \text { ) }\end{array}$ & 83.33333 & 0.428571 & 0.454545 & 0.47619 & 5 & 1 & 6 & 12 \\
\hline $\begin{array}{l}\text { Variable } 290 \text { (cols } 579 \& \\
580 \text { ) }\end{array}$ & 100 & 1 & 1 & 1 & 6 & 0 & 6 & 12 \\
\hline $\begin{array}{l}\text { Variable } 291 \text { (cols } 581 \& \\
582 \text { ) }\end{array}$ & 100 & 1 & 1 & 1 & 6 & 0 & 6 & 12 \\
\hline $\begin{array}{l}\text { Variable } 292 \text { (cols } 583 \text { \& } \\
584 \text { ) }\end{array}$ & 100 & 1 & 1 & 1 & 6 & 0 & 6 & 12 \\
\hline $\begin{array}{l}\text { Variable } 293 \text { (cols } 585 \text { \& } \\
586 \text { ) }\end{array}$ & 100 & 1 & 1 & 1 & 6 & 0 & 6 & 12 \\
\hline $\begin{array}{l}\text { Variable } 294 \text { (cols } 587 \text { \& } \\
588 \text { ) }\end{array}$ & 83.33333 & 0.707317 & 0.714286 & 0.731707 & 5 & 1 & 6 & 12 \\
\hline $\begin{array}{l}\text { Variable } 295 \text { (cols } 589 \& \\
590 \text { ) }\end{array}$ & 100 & 1 & 1 & 1 & 6 & 0 & 6 & 12 \\
\hline & & & & & & & & \\
\hline
\end{tabular}




\begin{tabular}{|l|l|l|l|l|l|l|l|l|}
\hline & $\begin{array}{l}\text { Percent } \\
\text { Agreement }\end{array}$ & Scott's Pi & Cohen's Kappa & $\begin{array}{l}\text { Krippendorf's } \\
\text { Alpha }\end{array}$ & N Agreements & $\begin{array}{l}\text { N } \\
\text { Disagreements }\end{array}$ & N Cases & N Decisions \\
\hline$* * *$ & & & $\begin{array}{l}\text { Krippendorf's } \\
\text { Alpha }\end{array}$ & 0.91004 & & & \\
\hline
\end{tabular}

Table B. 91: Inter- User Reliability

\begin{tabular}{|c|c|c|c|c|c|c|c|c|}
\hline & \begin{tabular}{|l|} 
Percent \\
Agreement
\end{tabular} & Scott's Pi & Cohen's Kappa & $\begin{array}{l}\text { Krippendorff's } \\
\text { Alpha }\end{array}$ & \begin{tabular}{|l|}
$\mathrm{N}$ \\
Agreements
\end{tabular} & \begin{tabular}{|l|}
$\mathrm{N}$ \\
Disagreements
\end{tabular} & N Cases & N Decisions \\
\hline Variable 1 (cols 1 \& 2) & $\begin{array}{r}66.6666 \\
7\end{array}$ & $\begin{array}{r}0.55140 \\
2\end{array}$ & 0.563636 & 0.570093 & 8 & 4 & 12 & 24 \\
\hline Variable 2 (cols $3 \& 4$ ) & $\begin{array}{r}91.6666 \\
7\end{array}$ & $\begin{array}{r}0.71084 \\
3\end{array}$ & 0.714286 & 0.722892 & 11 & 1 & 12 & 24 \\
\hline Variable 3 (cols 5 \& 6) & 100 & 1 & 1 & 1 & 12 & 0 & 12 & 24 \\
\hline Variable 4 (cols 7 \& 8) & $\begin{array}{r}91.6666 \\
7\end{array}$ & 0.04348 & 0 & 0 & 11 & 1 & 12 & 24 \\
\hline Variable 5 (cols $9 \& 10$ ) & 100 & 1 & 1 & 1 & 12 & 0 & 12 & 24 \\
\hline Variable 6 (cols $11 \& 12$ ) & 75 & $\begin{array}{r}0.56097 \\
6\end{array}$ & 0.571429 & 0.579268 & 9 & 3 & 12 & 24 \\
\hline Variable 7 (cols $13 \& 14)$ & $\begin{array}{r}91.6666 \\
7\end{array}$ & $\begin{array}{r}0.83892 \\
6\end{array}$ & 0.84 & 0.845638 & 11 & 1 & 12 & 24 \\
\hline Variable 8 (cols $15 \& 16)$ & 100 & 1 & 1 & 1 & 12 & 0 & 12 & 24 \\
\hline Variable 9 (cols $17 \& 18)$ & 100 & 1 & 1 & 1 & 12 & 0 & 12 & 24 \\
\hline Variable 10 (cols 19 \& 20) & 100 & 1 & 1 & 1 & 12 & 0 & 12 & 24 \\
\hline Variable 11 (cols $21 \& 22$ ) & $\begin{array}{r}83.3333 \\
3\end{array}$ & \begin{tabular}{|r|}
0.69230 \\
8
\end{tabular} & 0.692308 & 0.705128 & 10 & 2 & 12 & 24 \\
\hline Variable 12 (cols 23 \& 24) & 100 & 1 & 1 & 1 & 12 & 0 & 12 & 24 \\
\hline Variable 13 (cols 25 \& 26) & 100 & 1 & 1 & 1 & 12 & 0 & 12 & 24 \\
\hline Variable 14 (cols $27 \& 28$ ) & 100 & 1 & 1 & 1 & 12 & 0 & 12 & 24 \\
\hline Variable 15 (cols $29 \& 30$ ) & 100 & 1 & 1 & 1 & 12 & 0 & 12 & 24 \\
\hline Variable 16 (cols $31 \& 32$ ) & $\begin{array}{r}83.3333 \\
3\end{array}$ & $\begin{array}{r}0.70731 \\
7\end{array}$ & 0.714286 & 0.719512 & 10 & 2 & 12 & 24 \\
\hline Variable 17 (cols 33 \& 34) & 91.6666 & 0.74736 & 0.75 & 0.757895 & 11 & 1 & 12 & 24 \\
\hline
\end{tabular}




\begin{tabular}{|c|c|c|c|c|c|c|c|c|}
\hline & \begin{tabular}{|l|} 
Percent \\
Agreement
\end{tabular} & Scott's Pi & Cohen's Kappa & $\begin{array}{l}\text { Krippendorff's } \\
\text { Alpha }\end{array}$ & \begin{tabular}{|l|}
$\mathrm{N}$ \\
Agreements
\end{tabular} & \begin{tabular}{|l|}
$\mathrm{N}$ \\
Disagreements
\end{tabular} & N Cases & N Decisions \\
\hline & 7 & 8 & & & & & & \\
\hline Variable 18 (cols $35 \& 36$ ) & $\begin{array}{r}91.6666 \\
7\end{array}$ & 0.04348 & 0 & 0 & 11 & 1 & 12 & 24 \\
\hline Variable 19 (cols $37 \& 38$ ) & $\begin{array}{r}91.6666 \\
7\end{array}$ & 0.04348 & 0 & 0 & 11 & 1 & 12 & 24 \\
\hline Variable 20 (cols $39 \& 40$ ) & 100 & 1 & 1 & 1 & 12 & 0 & 12 & 24 \\
\hline Variable 21 (cols $41 \& 42$ ) & 100 & 1 & 1 & 1 & 12 & 0 & 12 & 24 \\
\hline Variable 22 (cols $43 \& 44$ ) & $\begin{array}{r}91.6666 \\
7\end{array}$ & $\begin{array}{r}0.74736 \\
8\end{array}$ & 0.75 & 0.757895 & 11 & 1 & 12 & 24 \\
\hline Variable 23 (cols $45 \& 46$ ) & 100 & 1 & 1 & 1 & 12 & 0 & 12 & 24 \\
\hline Variable 24 (cols $47 \& 48$ ) & 100 & 1 & 1 & 1 & 12 & 0 & 12 & 24 \\
\hline Variable 25 (cols $49 \& 50$ ) & 100 & 1 & 1 & 1 & 12 & 0 & 12 & 24 \\
\hline Variable 26 (cols 51 \& 52) & $\begin{array}{r}83.3333 \\
3\end{array}$ & $\begin{array}{r}0.70731 \\
7\end{array}$ & 0.714286 & 0.719512 & 10 & 2 & 12 & 24 \\
\hline Variable 27 (cols $53 \& 54$ ) & 100 & 1 & 1 & 1 & 12 & 0 & 12 & 24 \\
\hline Variable 28 (cols $55 \& 56$ ) & $\begin{array}{r}83.3333 \\
3\end{array}$ & $\begin{array}{r}0.70731 \\
7\end{array}$ & 0.714286 & 0.719512 & 10 & 2 & 12 & 24 \\
\hline Variable 29 (cols $57 \& 58$ ) & 100 & 1 & 1 & 1 & 12 & 0 & 12 & 24 \\
\hline Variable 30 (cols $59 \& 60$ ) & 100 & 1 & 1 & 1 & 12 & 0 & 12 & 24 \\
\hline Variable 31 (cols $61 \&$ 62) & $\begin{array}{r}83.3333 \\
3\end{array}$ & 0.4 & 0.428571 & 0.425 & 10 & 2 & 12 & 24 \\
\hline Variable 32 (cols $63 \& 64$ ) & 100 & 1 & 1 & 1 & 12 & 0 & 12 & 24 \\
\hline Variable 33 (cols 65 \& 66) & 100 & 1 & 1 & 1 & 12 & 0 & 12 & 24 \\
\hline Variable 34 (cols $67 \& 68$ ) & 100 & 1 & 1 & 1 & 12 & 0 & 12 & 24 \\
\hline Variable 35 (cols $69 \& 70$ ) & 100 & 1 & 1 & 1 & 12 & 0 & 12 & 24 \\
\hline Variable 36 (cols $71 \& 72$ ) & 100 & 1 & 1 & 1 & 12 & 0 & 12 & 24 \\
\hline Variable 37 (cols 73 \& 74) & 100 & 1 & 1 & 1 & 12 & 0 & 12 & 24 \\
\hline Variable 38 (cols 75 \& 76) & 100 & 1 & 1 & 1 & 12 & 0 & 12 & 24 \\
\hline Variable 39 (cols $77 \& 78$ ) & 100 & 1 & 1 & 1 & 12 & 0 & 12 & 24 \\
\hline Variable 40 (cols $79 \& 80$ ) & 100 & 1 & 1 & 1 & 12 & 0 & 12 & 24 \\
\hline
\end{tabular}




\begin{tabular}{|c|c|c|c|c|c|c|c|c|}
\hline & \begin{tabular}{|l|} 
Percent \\
Agreement
\end{tabular} & Scott's Pi & Cohen's Kappa & \begin{tabular}{|l|} 
Krippendorff's \\
Alpha
\end{tabular} & $\begin{array}{l}\mathrm{N} \\
\text { Agreements }\end{array}$ & $\mid \begin{array}{l}\mathrm{N} \\
\text { Disagreements }\end{array}$ & N Cases & N Decisions \\
\hline Variable 41 (cols 81 \& 82) & $\begin{array}{r}91.6666 \\
7\end{array}$ & $\begin{array}{r}0.74736 \\
8\end{array}$ & 0.75 & 0.757895 & 11 & 1 & 12 & 24 \\
\hline Variable 42 (cols 83 \& 84) & 100 & 1 & 1 & 1 & 12 & 0 & 12 & 24 \\
\hline Variable 43 (cols 85 \& 86) & 100 & 1 & 1 & 1 & 12 & 0 & 12 & 24 \\
\hline Variable 44 (cols $87 \& 88$ ) & 100 & 1 & 1 & 1 & 12 & 0 & 12 & 24 \\
\hline Variable 45 (cols $89 \& 90$ ) & 100 & 1 & 1 & 1 & 12 & 0 & 12 & 24 \\
\hline Variable 46 (cols 91 \& 92) & $\begin{array}{r}91.6666 \\
7\end{array}$ & $\begin{array}{r}0.61904 \\
8\end{array}$ & 0.625 & 0.634921 & 11 & 1 & 12 & 24 \\
\hline Variable 47 (cols 93 \& 94) & $\begin{array}{r}83.3333 \\
3\end{array}$ & 0.44186 & 0.454545 & 0.465116 & 10 & 2 & 12 & 24 \\
\hline Variable 48 (cols 95 \& 96) & 100 & 1 & 1 & 1 & 12 & 0 & 12 & 24 \\
\hline Variable 49 (cols 97 \& 98) & 100 & 1 & 1 & 1 & 12 & 0 & 12 & 24 \\
\hline Variable 50 (cols $99 \& 100$ ) & 100 & 1 & 1 & 1 & 12 & 0 & 12 & 24 \\
\hline $\begin{array}{l}\text { Variable } 51 \text { (cols } 101 \& \text { } \\
102)\end{array}$ & 100 & 1 & 1 & 1 & 12 & 0 & 12 & 24 \\
\hline $\begin{array}{l}\text { Variable } 52 \text { (cols } 103 \text { \& } \\
104 \text { ) }\end{array}$ & 100 & 1 & 1 & 1 & 12 & 0 & 12 & 24 \\
\hline $\begin{array}{l}\text { Variable } 53 \text { (cols } 105 \text { \& } \\
106 \text { ) }\end{array}$ & 100 & 1 & 1 & 1 & 12 & 0 & 12 & 24 \\
\hline $\begin{array}{l}\text { Variable } 54 \text { (cols } 107 \& \text { } \\
108)\end{array}$ & 100 & 1 & 1 & 1 & 12 & 0 & 12 & 24 \\
\hline $\begin{array}{l}\text { Variable } 55 \text { (cols } 109 \& \\
110)\end{array}$ & 100 & 1 & 1 & 1 & 12 & 0 & 12 & 24 \\
\hline $\begin{array}{l}\text { Variable } 56 \text { (cols } 111 \& \text { \& } \\
112 \text { ) }\end{array}$ & 100 & 1 & 1 & 1 & 12 & 0 & 12 & 24 \\
\hline $\begin{array}{l}\text { Variable } 57 \text { (cols } 113 \text { \& } \\
114 \text { ) }\end{array}$ & 100 & 1 & 1 & 1 & 12 & 0 & 12 & 24 \\
\hline $\begin{array}{l}\text { Variable } 58 \text { (cols } 115 \& \text { } \\
116)\end{array}$ & 100 & 1 & 1 & 1 & 12 & 0 & 12 & 24 \\
\hline $\begin{array}{l}\text { Variable } 59 \text { (cols } 117 \& \\
118)\end{array}$ & 100 & 1 & 1 & 1 & 12 & 0 & 12 & 24 \\
\hline $\begin{array}{l}\text { Variable } 60 \text { (cols } 119 \& \\
120)\end{array}$ & 100 & 1 & 1 & 1 & 12 & 0 & 12 & 24 \\
\hline
\end{tabular}




\begin{tabular}{|c|c|c|c|c|c|c|c|c|}
\hline & \begin{tabular}{|l|} 
Percent \\
Agreement
\end{tabular} & Scott's Pi & Cohen's Kappa & \begin{tabular}{|l|} 
Krippendorff's \\
Alpha
\end{tabular} & $\begin{array}{l}\mathrm{N} \\
\text { Agreements }\end{array}$ & $\begin{array}{l}\mathrm{N} \\
\text { Disagreements }\end{array}$ & N Cases & N Decisions \\
\hline $\begin{array}{l}\text { Variable } 61 \text { (cols } 121 \& \\
122 \text { ) }\end{array}$ & 100 & 1 & 1 & 1 & 12 & 0 & 12 & 24 \\
\hline $\begin{array}{l}\text { Variable } 62 \text { (cols } 123 \& \\
124)\end{array}$ & 100 & 1 & 1 & 1 & 12 & 0 & 12 & 24 \\
\hline $\begin{array}{l}\text { Variable } 63 \text { (cols } 125 \& \\
126)\end{array}$ & 100 & 1 & 1 & 1 & 12 & 0 & 12 & 24 \\
\hline $\begin{array}{l}\text { Variable } 64 \text { (cols } 127 \& \\
128 \text { ) }\end{array}$ & 100 & 1 & 1 & 1 & 12 & 0 & 12 & 24 \\
\hline $\begin{array}{l}\text { Variable } 65 \text { (cols } 129 \& \\
130 \text { ) }\end{array}$ & 100 & 1 & 1 & 1 & 12 & 0 & 12 & 24 \\
\hline $\begin{array}{l}\text { Variable } 66 \text { (cols } 131 \text { \& } \\
132 \text { ) }\end{array}$ & 100 & 1 & 1 & 1 & 12 & 0 & 12 & 24 \\
\hline $\begin{array}{l}\text { Variable } 67 \text { (cols } 133 \text { \& } \\
134 \text { ) }\end{array}$ & 100 & 1 & 1 & 1 & 12 & 0 & 12 & 24 \\
\hline $\begin{array}{l}\text { Variable } 68 \text { (cols } 135 \& \\
136 \text { ) }\end{array}$ & 100 & 1 & 1 & 1 & 12 & 0 & 12 & 24 \\
\hline $\begin{array}{l}\text { Variable } 69 \text { (cols } 137 \& \\
138 \text { ) }\end{array}$ & 100 & 1 & 1 & 1 & 12 & 0 & 12 & 24 \\
\hline $\begin{array}{l}\text { Variable } 70 \text { (cols } 139 \& \\
140)\end{array}$ & 100 & 1 & 1 & 1 & 12 & 0 & 12 & 24 \\
\hline $\begin{array}{l}\text { Variable } 71 \text { (cols } 141 \& \text { } \\
142 \text { ) }\end{array}$ & 100 & 1 & 1 & 1 & 12 & 0 & 12 & 24 \\
\hline $\begin{array}{l}\text { Variable } 72 \text { (cols } 143 \text { \& } \\
144)\end{array}$ & $\begin{array}{r}91.6666 \\
7\end{array}$ & $\begin{array}{r}0.81395 \\
3\end{array}$ & 0.815385 & 0.821705 & 11 & 1 & 12 & 24 \\
\hline $\begin{array}{l}\text { Variable } 73 \text { (cols } 145 \& \\
146 \text { ) }\end{array}$ & 100 & 1 & 1 & 1 & 12 & 0 & 12 & 24 \\
\hline $\begin{array}{l}\text { Variable } 74 \text { (cols } 147 \& \\
148 \text { ) }\end{array}$ & 100 & 1 & 1 & 1 & 12 & 0 & 12 & 24 \\
\hline $\begin{array}{l}\text { Variable } 75 \text { (cols } 149 \& \\
150)\end{array}$ & 100 & 1 & 1 & 1 & 12 & 0 & 12 & 24 \\
\hline $\begin{array}{l}\text { Variable } 76 \text { (cols } 151 \& \\
152 \text { ) }\end{array}$ & 100 & 1 & 1 & 1 & 12 & 0 & 12 & 24 \\
\hline $\begin{array}{l}\text { Variable } 77 \text { (cols } 153 \text { \& } \\
154)\end{array}$ & $\begin{array}{r}91.6666 \\
7\end{array}$ & $\begin{array}{r}0.81395 \\
3\end{array}$ & 0.815385 & 0.821705 & 11 & 1 & 12 & 24 \\
\hline Variable 78 (cols 155 \& & 91.6666 & 0.81395 & 0.815385 & 0.821705 & 11 & 1 & 12 & 24 \\
\hline
\end{tabular}




\begin{tabular}{|c|c|c|c|c|c|c|c|c|}
\hline & \begin{tabular}{|l|} 
Percent \\
Agreement
\end{tabular} & Scott's Pi & Cohen's Kappa & $\begin{array}{l}\text { Krippendorff's } \\
\text { Alpha }\end{array}$ & $\begin{array}{l}\mathrm{N} \\
\text { Agreements }\end{array}$ & $\begin{array}{l}\mathrm{N} \\
\text { Disagreements }\end{array}$ & N Cases & N Decisions \\
\hline 156) & 7 & 3 & & & & & & \\
\hline $\begin{array}{l}\text { Variable } 79 \text { (cols } 157 \& \\
158)\end{array}$ & 100 & 1 & 1 & 1 & 12 & 0 & 12 & 24 \\
\hline $\begin{array}{l}\text { Variable } 80 \text { (cols } 159 \& \\
160)\end{array}$ & 100 & 1 & 1 & 1 & 12 & 0 & 12 & 24 \\
\hline $\begin{array}{l}\text { Variable } 81 \text { (cols } 161 \& \\
162 \text { ) }\end{array}$ & 100 & 1 & 1 & 1 & 12 & 0 & 12 & 24 \\
\hline $\begin{array}{l}\text { Variable } 82 \text { (cols } 163 \& \\
164)\end{array}$ & $\begin{array}{r}91.6666 \\
7\end{array}$ & $\begin{array}{r}0.81395 \\
3\end{array}$ & 0.815385 & 0.821705 & 11 & 1 & 12 & 24 \\
\hline $\begin{array}{l}\text { Variable } 83 \text { (cols } 165 \& \\
166)\end{array}$ & $\begin{array}{r}91.6666 \\
7\end{array}$ & $\begin{array}{r}0.81395 \\
3\end{array}$ & 0.815385 & 0.821705 & 11 & 1 & 12 & 24 \\
\hline $\begin{array}{l}\text { Variable } 84 \text { (cols } 167 \& \\
168)\end{array}$ & 100 & 1 & 1 & 1 & 12 & 0 & 12 & 24 \\
\hline $\begin{array}{l}\text { Variable } 85 \text { (cols } 169 \& \\
170)\end{array}$ & 100 & 1 & 1 & 1 & 12 & 0 & 12 & 24 \\
\hline $\begin{array}{l}\text { Variable } 86 \text { (cols } 171 \& \\
172)\end{array}$ & $\begin{array}{r}83.3333 \\
3\end{array}$ & 0.625 & 0.625 & 0.640625 & 10 & 2 & 12 & 24 \\
\hline $\begin{array}{l}\text { Variable } 87 \text { (cols } 173 \& \\
174)\end{array}$ & 100 & 1 & 1 & 1 & 12 & 0 & 12 & 24 \\
\hline $\begin{array}{l}\text { Variable } 88 \text { (cols } 175 \& \\
176)\end{array}$ & 100 & 1 & 1 & 1 & 12 & 0 & 12 & 24 \\
\hline $\begin{array}{l}\text { Variable } 89 \text { (cols } 177 \& \\
178)\end{array}$ & 100 & 1 & 1 & 1 & 12 & 0 & 12 & 24 \\
\hline $\begin{array}{l}\text { Variable } 90 \text { (cols } 179 \& \\
180)\end{array}$ & 100 & 1 & 1 & 1 & 12 & 0 & 12 & 24 \\
\hline $\begin{array}{l}\text { Variable } 91 \text { (cols } 181 \& \text { } \\
182 \text { ) }\end{array}$ & $\begin{array}{r}83.3333 \\
3\end{array}$ & $\begin{array}{r}0.58620 \\
7\end{array}$ & 0.6 & 0.603448 & 10 & 2 & 12 & 24 \\
\hline $\begin{array}{l}\text { Variable } 92 \text { (cols } 183 \& \\
184 \text { ) }\end{array}$ & 100 & 1 & 1 & 1 & 12 & 0 & 12 & 24 \\
\hline $\begin{array}{l}\text { Variable } 93 \text { (cols } 185 \& \\
186)\end{array}$ & $\begin{array}{r}91.6666 \\
7\end{array}$ & $\begin{array}{r}0.61904 \\
8\end{array}$ & 0.625 & 0.634921 & 11 & 1 & 12 & 24 \\
\hline $\begin{array}{l}\text { Variable } 94 \text { (cols } 187 \& \\
188 \text { ) }\end{array}$ & $\begin{array}{r}91.6666 \\
7\end{array}$ & $\begin{array}{r}0.61904 \\
8\end{array}$ & 0.625 & 0.634921 & 11 & 1 & 12 & 24 \\
\hline $\begin{array}{l}\text { Variable } 95 \text { (cols } 189 \& \\
190)\end{array}$ & 100 & 1 & 1 & 1 & 12 & 0 & 12 & 24 \\
\hline
\end{tabular}




\begin{tabular}{|c|c|c|c|c|c|c|c|c|}
\hline & \begin{tabular}{|l|} 
Percent \\
Agreement
\end{tabular} & Scott's Pi & Cohen's Kappa & \begin{tabular}{|l|} 
Krippendorff's \\
Alpha
\end{tabular} & $\begin{array}{l}\mathrm{N} \\
\text { Agreements }\end{array}$ & $\begin{array}{l}\mathrm{N} \\
\text { Disagreements }\end{array}$ & N Cases & N Decisions \\
\hline $\begin{array}{l}\text { Variable } 96 \text { (cols } 191 \& \\
192 \text { ) }\end{array}$ & 100 & 1 & 1 & 1 & 12 & 0 & 12 & 24 \\
\hline $\begin{array}{l}\text { Variable } 97 \text { (cols } 193 \text { \& } \\
194 \text { ) }\end{array}$ & 100 & 1 & 1 & 1 & 12 & 0 & 12 & 24 \\
\hline $\begin{array}{l}\text { Variable } 98 \text { (cols } 195 \& \\
196)\end{array}$ & 100 & 1 & 1 & 1 & 12 & 0 & 12 & 24 \\
\hline $\begin{array}{l}\text { Variable } 99 \text { (cols } 197 \& \\
198 \text { ) }\end{array}$ & 100 & 1 & 1 & 1 & 12 & 0 & 12 & 24 \\
\hline $\begin{array}{l}\text { Variable } 100 \text { (cols } 199 \text { \& } \\
200)\end{array}$ & 100 & 1 & 1 & 1 & 12 & 0 & 12 & 24 \\
\hline $\begin{array}{l}\text { Variable } 101 \text { (cols } 201 \text { \& } \\
202 \text { ) }\end{array}$ & 100 & 1 & 1 & 1 & 12 & 0 & 12 & 24 \\
\hline $\begin{array}{l}\text { Variable } 102 \text { (cols } 203 \text { \& } \\
204 \text { ) }\end{array}$ & 100 & 1 & 1 & 1 & 12 & 0 & 12 & 24 \\
\hline $\begin{array}{l}\text { Variable } 103 \text { (cols } 205 \text { \& } \\
206 \text { ) }\end{array}$ & 100 & 1 & 1 & 1 & 12 & 0 & 12 & 24 \\
\hline $\begin{array}{l}\text { Variable } 104 \text { (cols } 207 \text { \& } \\
208 \text { ) }\end{array}$ & 100 & 1 & 1 & 1 & 12 & 0 & 12 & 24 \\
\hline $\begin{array}{l}\text { Variable } 105 \text { (cols } 209 \& \\
210 \text { ) }\end{array}$ & 100 & 1 & 1 & 1 & 12 & 0 & 12 & 24 \\
\hline $\begin{array}{l}\text { Variable } 106 \text { (cols } 211 \& \text { \& } \\
212 \text { ) }\end{array}$ & 100 & 1 & 1 & 1 & 12 & 0 & 12 & 24 \\
\hline $\begin{array}{l}\text { Variable } 107 \text { (cols } 213 \text { \& } \\
214 \text { ) }\end{array}$ & 100 & 1 & 1 & 1 & 12 & 0 & 12 & 24 \\
\hline $\begin{array}{l}\text { Variable } 108 \text { (cols } 215 \text { \& } \\
216 \text { ) }\end{array}$ & 100 & 1 & 1 & 1 & 12 & 0 & 12 & 24 \\
\hline $\begin{array}{l}\text { Variable } 109 \text { (cols } 217 \& \text { } \\
218 \text { ) }\end{array}$ & 100 & 1 & 1 & 1 & 12 & 0 & 12 & 24 \\
\hline $\begin{array}{l}\text { Variable } 110 \text { (cols } 219 \& \text { \& } \\
220 \text { ) }\end{array}$ & 100 & 1 & 1 & 1 & 12 & 0 & 12 & 24 \\
\hline $\begin{array}{l}\text { Variable } 111 \text { (cols } 221 \& \text { \& } \\
222 \text { ) }\end{array}$ & 100 & 1 & 1 & 1 & 12 & 0 & 12 & 24 \\
\hline $\begin{array}{l}\text { Variable } 112 \text { (cols } 223 \& \\
224 \text { ) }\end{array}$ & 100 & 1 & 1 & 1 & 12 & 0 & 12 & 24 \\
\hline Variable 113 (cols 225 \& & 100 & 1 & 1 & 1 & 12 & 0 & 12 & 24 \\
\hline
\end{tabular}




\begin{tabular}{|c|c|c|c|c|c|c|c|c|}
\hline & \begin{tabular}{|l|l|} 
Percent \\
Agreement
\end{tabular} & Scott's Pi & Cohen's Kappa & \begin{tabular}{|l|} 
Krippendorf's \\
Alpha
\end{tabular} & \begin{tabular}{|l|} 
N \\
Agreements
\end{tabular} & \begin{tabular}{|l|}
$N$ \\
Disagreements
\end{tabular} & $N$ Cases & N Decisions \\
\hline 226) & & & & & & & & \\
\hline $\begin{array}{l}\text { Variable } 114 \text { (cols } 227 \& \text { } \\
\text { 228) }\end{array}$ & 100 & 1 & 1 & 1 & 12 & 0 & 12 & 24 \\
\hline $\begin{array}{l}\text { Variable } 115 \text { (cols } 229 \& \\
\text { 230) }\end{array}$ & 100 & 1 & 1 & 1 & 12 & 0 & 12 & 24 \\
\hline $\begin{array}{l}\text { Variable } 116 \text { (cols } 231 \& \\
232 \text { ) }\end{array}$ & $\begin{array}{r}83.3333 \\
3\end{array}$ & 0.625 & 0.625 & 0.640625 & 10 & 2 & 12 & 24 \\
\hline $\begin{array}{l}\text { Variable } 117 \text { (cols } 233 \& \text { } \\
234 \text { ) }\end{array}$ & $\begin{array}{r}83.3333 \\
3\end{array}$ & 0.625 & 0.625 & 0.640625 & 10 & 2 & 12 & 24 \\
\hline $\begin{array}{l}\text { Variable } 118 \text { (cols } 235 \& \\
236)\end{array}$ & 100 & 1 & 1 & 1 & 12 & 0 & 12 & 24 \\
\hline $\begin{array}{l}\text { Variable } 119 \text { (cols } 237 \& \\
\text { 238) }\end{array}$ & 100 & 1 & 1 & 1 & 12 & 0 & 12 & 24 \\
\hline $\begin{array}{l}\text { Variable } 120 \text { (cols } 239 \& \\
240 \text { ) }\end{array}$ & $\begin{array}{r}91.6666 \\
7\end{array}$ & 0.04348 & 0 & 0 & 11 & 1 & 12 & 24 \\
\hline $\begin{array}{l}\text { Variable } 121 \text { (cols } 241 \& \\
\text { 242) }\end{array}$ & $\begin{array}{r}58.3333 \\
3\end{array}$ & $\begin{array}{r}0.35135 \\
1\end{array}$ & 0.361702 & 0.378378 & 7 & 5 & 12 & 24 \\
\hline $\begin{array}{l}\text { Variable } 122 \text { (cols } 243 \& \\
\text { 244) }\end{array}$ & $\begin{array}{r}66.6666 \\
7\end{array}$ & $\begin{array}{r}0.52941 \\
2\end{array}$ & 0.538462 & 0.54902 & 8 & 4 & 12 & 24 \\
\hline $\begin{array}{l}\text { Variable } 123 \text { (cols } 245 \& \text { } \\
246 \text { ) }\end{array}$ & 100 & 1 & 1 & 1 & 12 & 0 & 12 & 24 \\
\hline $\begin{array}{l}\text { Variable } 124 \text { (cols } 247 \& \text { } \\
248 \text { ) }\end{array}$ & 100 & 1 & 1 & 1 & 12 & 0 & 12 & 24 \\
\hline $\begin{array}{l}\text { Variable } 125 \text { (cols } 249 \& \\
250 \text { ) }\end{array}$ & 100 & 1 & 1 & 1 & 12 & 0 & 12 & 24 \\
\hline $\begin{array}{l}\text { Variable } 126 \text { (cols } 251 \& \\
252 \text { ) }\end{array}$ & $\begin{array}{r}83.3333 \\
3\end{array}$ & $\begin{array}{r}0.65217 \\
4\end{array}$ & 0.657143 & 0.666667 & 10 & 2 & 12 & 24 \\
\hline $\begin{array}{l}\text { Variable } 127 \text { (cols } 253 \& \\
254 \text { ) }\end{array}$ & $\begin{array}{r}91.6666 \\
7\end{array}$ & $\begin{array}{r}0.63076 \\
9\end{array}$ & 0.636364 & 0.646154 & 11 & 1 & 12 & 24 \\
\hline $\begin{array}{l}\text { Variable } 128 \text { (cols } 255 \& \text { } \\
256)\end{array}$ & 100 & 1 & 1 & 1 & 12 & 0 & 12 & 24 \\
\hline $\begin{array}{l}\text { Variable } 129 \text { (cols } 257 \text { \& } \\
258 \text { ) }\end{array}$ & $\begin{array}{r}91.6666 \\
7\end{array}$ & 0.04348 & 0 & 0 & 11 & 1 & 12 & 24 \\
\hline $\begin{array}{l}\text { Variable } 130 \text { (cols } 259 \& \\
260)\end{array}$ & 100 & 1 & 1 & 1 & 12 & 0 & 12 & 24 \\
\hline
\end{tabular}




\begin{tabular}{|c|c|c|c|c|c|c|c|c|}
\hline & $\begin{array}{l}\text { Percent } \\
\text { Agreement }\end{array}$ & Scott's Pi & Cohen's Kappa & \begin{tabular}{|l|} 
Krippendorff's \\
Alpha
\end{tabular} & $\begin{array}{l}\mathrm{N} \\
\text { Agreements }\end{array}$ & $\begin{array}{l}\mathrm{N} \\
\text { Disagreements }\end{array}$ & N Cases & N Decisions \\
\hline $\begin{array}{l}\text { Variable } 131 \text { (cols } 261 \& \\
262 \text { ) }\end{array}$ & 100 & 1 & 1 & 1 & 12 & 0 & 12 & 24 \\
\hline $\begin{array}{l}\text { Variable } 132 \text { (cols } 263 \text { \& } \\
\text { 264) }\end{array}$ & 100 & 1 & 1 & 1 & 12 & 0 & 12 & 24 \\
\hline $\begin{array}{l}\text { Variable } 133 \text { (cols } 265 \& \\
266 \text { ) }\end{array}$ & 100 & 1 & 1 & 1 & 12 & 0 & 12 & 24 \\
\hline $\begin{array}{l}\text { Variable } 134 \text { (cols } 267 \& \\
268 \text { ) }\end{array}$ & 100 & 1 & 1 & 1 & 12 & 0 & 12 & 24 \\
\hline $\begin{array}{l}\text { Variable } 135 \text { (cols } 269 \& \\
270)\end{array}$ & 100 & 1 & 1 & 1 & 12 & 0 & 12 & 24 \\
\hline $\begin{array}{l}\text { Variable } 136 \text { (cols } 271 \& \text { \& } \\
272 \text { ) }\end{array}$ & $\begin{array}{r}91.6666 \\
7\end{array}$ & $\begin{array}{r}0.63076 \\
9\end{array}$ & 0.636364 & 0.646154 & 11 & 1 & 12 & 24 \\
\hline $\begin{array}{l}\text { Variable } 137 \text { (cols } 273 \& \\
274 \text { ) }\end{array}$ & $\begin{array}{r}83.3333 \\
3\end{array}$ & $\begin{array}{r}0.27272 \\
7\end{array}$ & 0.294118 & 0.30303 & 10 & 2 & 12 & 24 \\
\hline $\begin{array}{l}\text { Variable } 138 \text { (cols } 275 \& \\
276 \text { ) }\end{array}$ & $\begin{array}{r}91.6666 \\
7\end{array}$ & 0.04348 & 0 & 0 & 11 & 1 & 12 & 24 \\
\hline $\begin{array}{l}\text { Variable } 139 \text { (cols } 277 \& \text { \& } \\
278 \text { ) }\end{array}$ & 100 & 1 & 1 & 1 & 12 & 0 & 12 & 24 \\
\hline $\begin{array}{l}\text { Variable } 140 \text { (cols } 279 \& \\
280)\end{array}$ & 100 & 1 & 1 & 1 & 12 & 0 & 12 & 24 \\
\hline $\begin{array}{l}\text { Variable } 141 \text { (cols } 281 \& \\
282 \text { ) }\end{array}$ & 100 & 1 & 1 & 1 & 12 & 0 & 12 & 24 \\
\hline $\begin{array}{l}\text { Variable } 142 \text { (cols } 283 \& \\
284 \text { ) }\end{array}$ & 100 & 1 & 1 & 1 & 12 & 0 & 12 & 24 \\
\hline $\begin{array}{l}\text { Variable } 143 \text { (cols } 285 \& \\
286 \text { ) }\end{array}$ & 100 & 1 & 1 & 1 & 12 & 0 & 12 & 24 \\
\hline $\begin{array}{l}\text { Variable } 144 \text { (cols } 287 \& \\
288 \text { ) }\end{array}$ & 100 & 1 & 1 & 1 & 12 & 0 & 12 & 24 \\
\hline $\begin{array}{l}\text { Variable } 145 \text { (cols } 289 \& \\
290 \text { ) }\end{array}$ & $\begin{array}{r}91.6666 \\
7\end{array}$ & 0.04348 & 0 & 0 & 11 & 1 & 12 & 24 \\
\hline $\begin{array}{l}\text { Variable } 146 \text { (cols } 291 \& \text { \& } \\
292 \text { ) }\end{array}$ & $\begin{array}{r}91.6666 \\
7\end{array}$ & $\begin{array}{r}0.46666 \\
7\end{array}$ & 0.478261 & 0.488889 & 11 & 1 & 12 & 24 \\
\hline $\begin{array}{l}\text { Variable } 147 \text { (cols } 293 \text { \& } \\
294 \text { ) }\end{array}$ & 100 & 1 & 1 & 1 & 12 & 0 & 12 & 24 \\
\hline Variable 148 (cols 295 \& & 91.6666 & 0.46666 & 0.478261 & 0.488889 & 11 & 1 & 12 & 24 \\
\hline
\end{tabular}




\begin{tabular}{|c|c|c|c|c|c|c|c|c|}
\hline & \begin{tabular}{|l|} 
Percent \\
Agreement
\end{tabular} & Scott's Pi & Cohen's Kappa & $\begin{array}{l}\text { Krippendorff's } \\
\text { Alpha }\end{array}$ & \begin{tabular}{|l|} 
N \\
Agreements
\end{tabular} & \begin{tabular}{|l|}
$N$ \\
Disagreements
\end{tabular} & $N$ Cases & N Decisions \\
\hline 296) & 7 & 7 & & & & & & \\
\hline $\begin{array}{l}\text { Variable } 149 \text { (cols } 297 \text { \& } \\
\text { 298) }\end{array}$ & $\begin{array}{r}91.6666 \\
7\end{array}$ & 0.04348 & 0 & 0 & 11 & 1 & 12 & 24 \\
\hline $\begin{array}{l}\text { Variable } 150 \text { (cols } 299 \& \\
300)\end{array}$ & $\begin{array}{r}91.6666 \\
7\end{array}$ & 0.04348 & 0 & 0 & 11 & 1 & 12 & 24 \\
\hline $\begin{array}{l}\text { Variable } 151 \text { (cols } 301 \& \\
302 \text { ) }\end{array}$ & 100 & 1 & 1 & 1 & 12 & 0 & 12 & 24 \\
\hline $\begin{array}{l}\text { Variable } 152 \text { (cols } 303 \text { \& } \\
\text { 304) }\end{array}$ & 100 & 1 & 1 & 1 & 12 & 0 & 12 & 24 \\
\hline $\begin{array}{l}\text { Variable } 153 \text { (cols } 305 \text { \& } \\
306)\end{array}$ & $\begin{array}{r}91.6666 \\
7\end{array}$ & 0.04348 & 0 & 0 & 11 & 1 & 12 & 24 \\
\hline $\begin{array}{l}\text { Variable } 154 \text { (cols } 307 \text { \& } \\
308 \text { ) }\end{array}$ & 100 & 1 & 1 & 1 & 12 & 0 & 12 & 24 \\
\hline $\begin{array}{l}\text { Variable } 155 \text { (cols } 309 \& \\
310)\end{array}$ & $\begin{array}{r}91.6666 \\
7\end{array}$ & $\begin{array}{r}0.88516 \\
7\end{array}$ & 0.885714 & 0.889952 & 11 & 1 & 12 & 24 \\
\hline $\begin{array}{l}\text { Variable } 156 \text { (cols } 311 \text { \& } \\
312 \text { ) }\end{array}$ & 100 & 1 & 1 & 1 & 12 & 0 & 12 & 24 \\
\hline $\begin{array}{l}\text { Variable } 157 \text { (cols } 313 \text { \& } \\
\text { 314) }\end{array}$ & 100 & 1 & 1 & 1 & 12 & 0 & 12 & 24 \\
\hline $\begin{array}{l}\text { Variable } 158 \text { (cols } 315 \text { \& } \\
316)\end{array}$ & 100 & 1 & 1 & 1 & 12 & 0 & 12 & 24 \\
\hline $\begin{array}{l}\text { Variable } 159 \text { (cols } 317 \text { \& } \\
318)\end{array}$ & 100 & 1 & 1 & 1 & 12 & 0 & 12 & 24 \\
\hline $\begin{array}{l}\text { Variable } 160 \text { (cols } 319 \& \\
320)\end{array}$ & 100 & 1 & 1 & 1 & 12 & 0 & 12 & 24 \\
\hline $\begin{array}{l}\text { Variable } 161 \text { (cols } 321 \& \\
322 \text { ) }\end{array}$ & 100 & 1 & 1 & 1 & 12 & 0 & 12 & 24 \\
\hline $\begin{array}{l}\text { Variable } 162 \text { (cols } 323 \& \\
324 \text { ) }\end{array}$ & $\begin{array}{r}91.6666 \\
7\end{array}$ & $\begin{array}{r}0.61904 \\
8\end{array}$ & 0.625 & 0.634921 & 11 & 1 & 12 & 24 \\
\hline $\begin{array}{l}\text { Variable } 163 \text { (cols } 325 \& \\
326 \text { ) }\end{array}$ & 100 & 1 & 1 & 1 & 12 & 0 & 12 & 24 \\
\hline $\begin{array}{l}\text { Variable } 164 \text { (cols } 327 \& \\
328 \text { ) }\end{array}$ & 100 & 1 & 1 & 1 & 12 & 0 & 12 & 24 \\
\hline $\begin{array}{l}\text { Variable } 165 \text { (cols } 329 \& \\
330 \text { ) }\end{array}$ & 100 & 1 & 1 & 1 & 12 & 0 & 12 & 24 \\
\hline
\end{tabular}




\begin{tabular}{|c|c|c|c|c|c|c|c|c|}
\hline & \begin{tabular}{|l|} 
Percent \\
Agreement
\end{tabular} & Scott's Pi & Cohen's Kappa & $\begin{array}{l}\text { Krippendorff's } \\
\text { Alpha }\end{array}$ & $\begin{array}{l}\mathrm{N} \\
\text { Agreements }\end{array}$ & $\mid \begin{array}{l}\mathrm{N} \\
\text { Disagreements }\end{array}$ & N Cases & N Decisions \\
\hline $\begin{array}{l}\text { Variable } 166 \text { (cols } 331 \text { \& } \\
332 \text { ) }\end{array}$ & $\begin{array}{r}83.3333 \\
3\end{array}$ & $\begin{array}{r}0.53846 \\
2\end{array}$ & 0.54717 & 0.557692 & 10 & 2 & 12 & 24 \\
\hline $\begin{array}{l}\text { Variable } 167 \text { (cols } 333 \text { \& } \\
334 \text { ) }\end{array}$ & $\begin{array}{r}58.3333 \\
3\end{array}$ & $\begin{array}{r}0.40886 \\
7\end{array}$ & 0.428571 & 0.433498 & 7 & 5 & 12 & 24 \\
\hline $\begin{array}{l}\text { Variable } 168 \text { (cols } 335 \text { \& } \\
336 \text { ) }\end{array}$ & $\begin{array}{r}83.3333 \\
3\end{array}$ & 0.44186 & 0.454545 & 0.465116 & 10 & 2 & 12 & 24 \\
\hline $\begin{array}{l}\text { Variable } 169 \text { (cols } 337 \text { \& } \\
338 \text { ) }\end{array}$ & 100 & 1 & 1 & 1 & 12 & 0 & 12 & 24 \\
\hline $\begin{array}{l}\text { Variable } 170 \text { (cols } 339 \text { \& } \\
340)\end{array}$ & 100 & 1 & 1 & 1 & 12 & 0 & 12 & 24 \\
\hline $\begin{array}{l}\text { Variable } 171 \text { (cols } 341 \text { \& } \\
342 \text { ) }\end{array}$ & 100 & 1 & 1 & 1 & 12 & 0 & 12 & 24 \\
\hline $\begin{array}{l}\text { Variable } 172 \text { (cols } 343 \text { \& } \\
344 \text { ) }\end{array}$ & $\begin{array}{r}83.3333 \\
3\end{array}$ & 0.44186 & 0.454545 & 0.465116 & 10 & 2 & 12 & 24 \\
\hline $\begin{array}{l}\text { Variable } 173 \text { (cols } 345 \text { \& } \\
346 \text { ) }\end{array}$ & $\begin{array}{r}83.3333 \\
3\end{array}$ & $\begin{array}{r}0.73333 \\
3\end{array}$ & 0.733333 & 0.744444 & 10 & 2 & 12 & 24 \\
\hline $\begin{array}{l}\text { Variable } 174 \text { (cols } 347 \& \\
348 \text { ) }\end{array}$ & 100 & 1 & 1 & 1 & 12 & 0 & 12 & 24 \\
\hline $\begin{array}{l}\text { Variable } 175 \text { (cols } 349 \& \\
350)\end{array}$ & 100 & 1 & 1 & 1 & 12 & 0 & 12 & 24 \\
\hline $\begin{array}{l}\text { Variable } 176 \text { (cols } 351 \text { \& } \\
352 \text { ) }\end{array}$ & $\begin{array}{r}91.6666 \\
7\end{array}$ & 0.04348 & 0 & 0 & 11 & 1 & 12 & 24 \\
\hline $\begin{array}{l}\text { Variable } 177 \text { (cols } 353 \text { \& } \\
354 \text { ) }\end{array}$ & 100 & 1 & 1 & 1 & 12 & 0 & 12 & 24 \\
\hline $\begin{array}{l}\text { Variable } 178 \text { (cols } 355 \text { \& } \\
356 \text { ) }\end{array}$ & 100 & 1 & 1 & 1 & 12 & 0 & 12 & 24 \\
\hline $\begin{array}{l}\text { Variable } 179 \text { (cols } 357 \text { \& } \\
358 \text { ) }\end{array}$ & $\begin{array}{r}91.6666 \\
7\end{array}$ & $\begin{array}{r}0.61904 \\
8\end{array}$ & 0.625 & 0.634921 & 11 & 1 & 12 & 24 \\
\hline $\begin{array}{l}\text { Variable } 180 \text { (cols } 359 \text { \& } \\
360 \text { ) }\end{array}$ & 100 & 1 & 1 & 1 & 12 & 0 & 12 & 24 \\
\hline $\begin{array}{l}\text { Variable } 181 \text { (cols } 361 \& \text { } \\
362 \text { ) }\end{array}$ & 100 & 1 & 1 & 1 & 12 & 0 & 12 & 24 \\
\hline $\begin{array}{l}\text { Variable } 182 \text { (cols } 363 \& \\
364 \text { ) }\end{array}$ & 100 & 1 & 1 & 1 & 12 & 0 & 12 & 24 \\
\hline Variable 183 (cols 365 \& & 100 & 1 & 1 & 1 & 12 & 0 & 12 & 24 \\
\hline
\end{tabular}




\begin{tabular}{|c|c|c|c|c|c|c|c|c|}
\hline & \begin{tabular}{|l|} 
Percent \\
Agreement
\end{tabular} & Scott's Pi & Cohen's Kappa & \begin{tabular}{|l|} 
Krippendorf's \\
Alpha
\end{tabular} & \begin{tabular}{|l|}
$\mathrm{N}$ \\
Agreements
\end{tabular} & \begin{tabular}{|l|}
$N$ \\
Disagreements
\end{tabular} & N Cases & N Decisions \\
\hline \multicolumn{9}{|l|}{ 366) } \\
\hline $\begin{array}{l}\text { Variable } 184 \text { (cols } 367 \& \\
368 \text { ) }\end{array}$ & 100 & 1 & 1 & 1 & 12 & 0 & 12 & 24 \\
\hline $\begin{array}{l}\text { Variable } 185 \text { (cols } 369 \& \\
370 \text { ) }\end{array}$ & $\begin{array}{r}91.6666 \\
7\end{array}$ & $\begin{array}{r}- \\
0.04348\end{array}$ & 0 & 0 & 11 & 1 & 12 & 24 \\
\hline $\begin{array}{l}\text { Variable } 186 \text { (cols } 371 \& \\
372 \text { ) }\end{array}$ & $\begin{array}{r}66.6666 \\
7\end{array}$ & $\begin{array}{r}0.53170 \\
7\end{array}$ & 0.542857 & 0.55122 & 8 & 4 & 12 & 24 \\
\hline $\begin{array}{l}\text { Variable } 187 \text { (cols } 373 \& \text { } \\
\text { 374) }\end{array}$ & $\begin{array}{r}83.3333 \\
3\end{array}$ & $\begin{array}{r}0.73333 \\
3\end{array}$ & 0.733333 & 0.744444 & 10 & 2 & 12 & 24 \\
\hline $\begin{array}{l}\text { Variable } 188 \text { (cols } 375 \& \text { } \\
376 \text { ) }\end{array}$ & 100 & 1 & 1 & 1 & 12 & 0 & 12 & 24 \\
\hline $\begin{array}{l}\text { Variable } 189 \text { (cols } 377 \& \\
378 \text { ) }\end{array}$ & 100 & 1 & 1 & 1 & 12 & 0 & 12 & 24 \\
\hline $\begin{array}{l}\text { Variable } 190 \text { (cols } 379 \& \\
380 \text { ) }\end{array}$ & $\begin{array}{r}83.3333 \\
3\end{array}$ & $\begin{array}{r}0.73333 \\
3\end{array}$ & 0.733333 & 0.744444 & 10 & 2 & 12 & 24 \\
\hline $\begin{array}{l}\text { Variable } 191 \text { (cols } 381 \text { \& } \\
382 \text { ) }\end{array}$ & $\begin{array}{r}91.6666 \\
7\end{array}$ & $\begin{array}{r}0.86440 \\
7\end{array}$ & 0.865169 & 0.870056 & 11 & 1 & 12 & 24 \\
\hline $\begin{array}{l}\text { Variable } 192 \text { (cols } 383 \text { \& } \\
384 \text { ) }\end{array}$ & $\begin{array}{r}83.3333 \\
3\end{array}$ & $\begin{array}{r}0.72413 \\
8\end{array}$ & 0.727273 & 0.735632 & 10 & 2 & 12 & 24 \\
\hline $\begin{array}{l}\text { Variable } 193 \text { (cols } 385 \text { \& } \\
386 \text { ) }\end{array}$ & $\begin{array}{r}91.6666 \\
7\end{array}$ & \begin{tabular}{|r|}
0.61904 \\
8
\end{tabular} & 0.625 & 0.634921 & 11 & 1 & 12 & 24 \\
\hline $\begin{array}{l}\text { Variable } 194 \text { (cols } 387 \& \\
\text { 388) }\end{array}$ & 100 & 1 & 1 & 1 & 12 & 0 & 12 & 24 \\
\hline $\begin{array}{l}\text { Variable } 195 \text { (cols } 389 \text { \& } \\
390)\end{array}$ & 100 & 1 & 1 & 1 & 12 & 0 & 12 & 24 \\
\hline $\begin{array}{l}\text { Variable } 196 \text { (cols } 391 \text { \& } \\
392 \text { ) }\end{array}$ & 100 & 1 & 1 & 1 & 12 & 0 & 12 & 24 \\
\hline $\begin{array}{l}\text { Variable } 197 \text { (cols } 393 \text { \& } \\
\text { 394) }\end{array}$ & 100 & 1 & 1 & 1 & 12 & 0 & 12 & 24 \\
\hline $\begin{array}{l}\text { Variable } 198 \text { (cols } 395 \& \\
\text { 396) }\end{array}$ & 100 & 1 & 1 & 1 & 12 & 0 & 12 & 24 \\
\hline $\begin{array}{l}\text { Variable } 199 \text { (cols } 397 \& \\
\text { 398) }\end{array}$ & 100 & 1 & 1 & 1 & 12 & 0 & 12 & 24 \\
\hline $\begin{array}{l}\text { Variable } 200 \text { (cols } 399 \& \\
400 \text { ) }\end{array}$ & 100 & 1 & 1 & 1 & 12 & 0 & 12 & 24 \\
\hline
\end{tabular}




\begin{tabular}{|c|c|c|c|c|c|c|c|c|}
\hline & $\begin{array}{l}\text { Percent } \\
\text { Agreement }\end{array}$ & Scott's Pi & Cohen's Kappa & \begin{tabular}{|l|} 
Krippendorff's \\
Alpha
\end{tabular} & $\begin{array}{l}\mathrm{N} \\
\text { Agreements }\end{array}$ & $\begin{array}{l}\mathrm{N} \\
\text { Disagreements }\end{array}$ & N Cases & N Decisions \\
\hline $\begin{array}{l}\text { Variable } 201 \text { (cols } 401 \text { \& } \\
402 \text { ) }\end{array}$ & 100 & 1 & 1 & 1 & 12 & 0 & 12 & 24 \\
\hline $\begin{array}{l}\text { Variable } 202 \text { (cols } 403 \text { \& } \\
404 \text { ) }\end{array}$ & 100 & 1 & 1 & 1 & 12 & 0 & 12 & 24 \\
\hline $\begin{array}{l}\text { Variable } 203 \text { (cols } 405 \text { \& } \\
406 \text { ) }\end{array}$ & 100 & 1 & 1 & 1 & 12 & 0 & 12 & 24 \\
\hline $\begin{array}{l}\text { Variable } 204 \text { (cols } 407 \text { \& } \\
408 \text { ) }\end{array}$ & 100 & 1 & 1 & 1 & 12 & 0 & 12 & 24 \\
\hline $\begin{array}{l}\text { Variable } 205 \text { (cols } 409 \text { \& } \\
410)\end{array}$ & 100 & 1 & 1 & 1 & 12 & 0 & 12 & 24 \\
\hline $\begin{array}{l}\text { Variable } 206 \text { (cols } 411 \text { \& } \\
412 \text { ) }\end{array}$ & $\begin{array}{r}91.6666 \\
7\end{array}$ & $\begin{array}{r}0.81395 \\
3\end{array}$ & 0.815385 & 0.821705 & 11 & 1 & 12 & 24 \\
\hline $\begin{array}{l}\text { Variable } 207 \text { (cols } 413 \text { \& } \\
414 \text { ) }\end{array}$ & $\begin{array}{r}83.3333 \\
3\end{array}$ & $\begin{array}{r}0.27272 \\
7\end{array}$ & 0.294118 & 0.30303 & 10 & 2 & 12 & 24 \\
\hline $\begin{array}{l}\text { Variable } 208 \text { (cols } 415 \text { \& } \\
416 \text { ) }\end{array}$ & 100 & 1 & 1 & 1 & 12 & 0 & 12 & 24 \\
\hline $\begin{array}{l}\text { Variable } 209 \text { (cols } 417 \text { \& } \\
418 \text { ) }\end{array}$ & 100 & 1 & 1 & 1 & 12 & 0 & 12 & 24 \\
\hline $\begin{array}{l}\text { Variable } 210 \text { (cols } 419 \text { \& } \\
420)\end{array}$ & 100 & 1 & 1 & 1 & 12 & 0 & 12 & 24 \\
\hline $\begin{array}{l}\text { Variable } 211 \text { (cols } 421 \text { \& } \\
422 \text { ) }\end{array}$ & $\begin{array}{r}91.6666 \\
7\end{array}$ & 0.04348 & 0 & 0 & 11 & 1 & 12 & 24 \\
\hline $\begin{array}{l}\text { Variable } 212 \text { (cols } 423 \text { \& } \\
424)\end{array}$ & $\begin{array}{r}91.6666 \\
7\end{array}$ & 0.04348 & 0 & 0 & 11 & 1 & 12 & 24 \\
\hline $\begin{array}{l}\text { Variable } 213 \text { (cols } 425 \& \\
426 \text { ) }\end{array}$ & 100 & 1 & 1 & 1 & 12 & 0 & 12 & 24 \\
\hline $\begin{array}{l}\text { Variable } 214 \text { (cols } 427 \& \text { } \\
428 \text { ) }\end{array}$ & 100 & 1 & 1 & 1 & 12 & 0 & 12 & 24 \\
\hline $\begin{array}{l}\text { Variable } 215 \text { (cols } 429 \& \\
430)\end{array}$ & 100 & 1 & 1 & 1 & 12 & 0 & 12 & 24 \\
\hline $\begin{array}{l}\text { Variable } 216 \text { (cols } 431 \& \\
432 \text { ) }\end{array}$ & 100 & 1 & 1 & 1 & 12 & 0 & 12 & 24 \\
\hline $\begin{array}{l}\text { Variable } 217 \text { (cols } 433 \text { \& } \\
434 \text { ) }\end{array}$ & 100 & 1 & 1 & 1 & 12 & 0 & 12 & 24 \\
\hline Variable 218 (cols 435 \& & 100 & 1 & 1 & 1 & 12 & 0 & 12 & 24 \\
\hline
\end{tabular}




\begin{tabular}{|c|c|c|c|c|c|c|c|c|}
\hline & \begin{tabular}{|l|} 
Percent \\
Agreement
\end{tabular} & Scott's Pi & Cohen's Kappa & \begin{tabular}{|l|} 
Krippendorff's \\
Alpha
\end{tabular} & \begin{tabular}{|l|}
$\mathrm{N}$ \\
Agreements
\end{tabular} & \begin{tabular}{|l|}
$N$ \\
Disagreements
\end{tabular} & N Cases & N Decisions \\
\hline 436) & & & & & & & & \\
\hline $\begin{array}{l}\text { Variable } 219 \text { (cols } 437 \& \\
438 \text { ) }\end{array}$ & 100 & 1 & 1 & 1 & 12 & 0 & 12 & 24 \\
\hline $\begin{array}{l}\text { Variable } 220 \text { (cols } 439 \& \\
440 \text { ) }\end{array}$ & 100 & 1 & 1 & 1 & 12 & 0 & 12 & 24 \\
\hline $\begin{array}{l}\text { Variable } 221 \text { (cols } 441 \text { \& } \\
442 \text { ) }\end{array}$ & 100 & 1 & 1 & 1 & 12 & 0 & 12 & 24 \\
\hline $\begin{array}{l}\text { Variable } 222 \text { (cols } 443 \& \text { \& } \\
444 \text { ) }\end{array}$ & 100 & 1 & 1 & 1 & 12 & 0 & 12 & 24 \\
\hline $\begin{array}{l}\text { Variable } 223 \text { (cols } 445 \& \\
446)\end{array}$ & 100 & 1 & 1 & 1 & 12 & 0 & 12 & 24 \\
\hline $\begin{array}{l}\text { Variable } 224 \text { (cols } 447 \& \\
448 \text { ) }\end{array}$ & 100 & 1 & 1 & 1 & 12 & 0 & 12 & 24 \\
\hline $\begin{array}{l}\text { Variable } 225 \text { (cols } 449 \& \\
450 \text { ) }\end{array}$ & 100 & 1 & 1 & 1 & 12 & 0 & 12 & 24 \\
\hline $\begin{array}{l}\text { Variable } 226 \text { (cols } 451 \& \\
452 \text { ) }\end{array}$ & 100 & 1 & 1 & 1 & 12 & 0 & 12 & 24 \\
\hline $\begin{array}{l}\text { Variable } 227 \text { (cols } 453 \text { \& } \\
454)\end{array}$ & 100 & 1 & 1 & 1 & 12 & 0 & 12 & 24 \\
\hline $\begin{array}{l}\text { Variable } 228 \text { (cols } 455 \& \\
456)\end{array}$ & 100 & 1 & 1 & 1 & 12 & 0 & 12 & 24 \\
\hline $\begin{array}{l}\text { Variable } 229 \text { (cols } 457 \text { \& } \\
458 \text { ) }\end{array}$ & 100 & 1 & 1 & 1 & 12 & 0 & 12 & 24 \\
\hline $\begin{array}{l}\text { Variable } 230 \text { (cols } 459 \text { \& } \\
460 \text { ) }\end{array}$ & 100 & 1 & 1 & 1 & 12 & 0 & 12 & 24 \\
\hline $\begin{array}{l}\text { Variable } 231 \text { (cols } 461 \text { \& } \\
462 \text { ) }\end{array}$ & 100 & 1 & 1 & 1 & 12 & 0 & 12 & 24 \\
\hline $\begin{array}{l}\text { Variable } 232 \text { (cols } 463 \text { \& } \\
464 \text { ) }\end{array}$ & 100 & 1 & 1 & 1 & 12 & 0 & 12 & 24 \\
\hline $\begin{array}{l}\text { Variable } 233 \text { (cols } 465 \& \\
466 \text { ) }\end{array}$ & 100 & 1 & 1 & 1 & 12 & 0 & 12 & 24 \\
\hline $\begin{array}{l}\text { Variable } 234 \text { (cols } 467 \& \\
468 \text { ) }\end{array}$ & 100 & 1 & 1 & 1 & 12 & 0 & 12 & 24 \\
\hline $\begin{array}{l}\text { Variable } 235 \text { (cols } 469 \& \\
470 \text { ) }\end{array}$ & 100 & 1 & 1 & 1 & 12 & 0 & 12 & 24 \\
\hline
\end{tabular}




\begin{tabular}{|c|c|c|c|c|c|c|c|c|}
\hline & \begin{tabular}{|l|} 
Percent \\
Agreement
\end{tabular} & Scott's Pi & Cohen's Kappa & \begin{tabular}{|l|} 
Krippendorff's \\
Alpha
\end{tabular} & $\begin{array}{l}\mathrm{N} \\
\text { Agreements }\end{array}$ & $\begin{array}{l}\mathrm{N} \\
\text { Disagreements }\end{array}$ & N Cases & N Decisions \\
\hline $\begin{array}{l}\text { Variable } 236 \text { (cols } 471 \text { \& } \\
472 \text { ) }\end{array}$ & $\begin{array}{r}66.6666 \\
7\end{array}$ & 0.6 & 0.603306 & 0.616667 & 8 & 4 & 12 & 24 \\
\hline $\begin{array}{l}\text { Variable } 237 \text { (cols } 473 \text { \& } \\
474 \text { ) }\end{array}$ & $\begin{array}{r}66.6666 \\
7\end{array}$ & $\begin{array}{r}0.56363 \\
6\end{array}$ & 0.567568 & 0.581818 & 8 & 4 & 12 & 24 \\
\hline $\begin{array}{l}\text { Variable } 238 \text { (cols } 475 \& \text { } \\
476 \text { ) }\end{array}$ & 75 & $\begin{array}{r}0.36283 \\
2\end{array}$ & 0.4 & 0.389381 & 9 & 3 & 12 & 24 \\
\hline $\begin{array}{l}\text { Variable } 239 \text { (cols } 477 \& \\
478 \text { ) }\end{array}$ & 100 & 1 & 1 & 1 & 12 & 0 & 12 & 24 \\
\hline $\begin{array}{l}\text { Variable } 240 \text { (cols } 479 \text { \& } \\
480 \text { ) }\end{array}$ & 100 & 1 & 1 & 1 & 12 & 0 & 12 & 24 \\
\hline $\begin{array}{l}\text { Variable } 241 \text { (cols } 481 \& \text { } \\
482 \text { ) }\end{array}$ & 100 & 1 & 1 & 1 & 12 & 0 & 12 & 24 \\
\hline $\begin{array}{l}\text { Variable } 242 \text { (cols } 483 \text { \& } \\
484 \text { ) }\end{array}$ & $\begin{array}{r}91.6666 \\
7\end{array}$ & 0.04348 & 0 & 0 & 11 & 1 & 12 & 24 \\
\hline $\begin{array}{l}\text { Variable } 243 \text { (cols } 485 \& \\
486 \text { ) }\end{array}$ & 100 & 1 & 1 & 1 & 12 & 0 & 12 & 24 \\
\hline $\begin{array}{l}\text { Variable } 244 \text { (cols } 487 \& \\
488 \text { ) }\end{array}$ & 100 & 1 & 1 & 1 & 12 & 0 & 12 & 24 \\
\hline $\begin{array}{l}\text { Variable } 245 \text { (cols } 489 \& \\
490)\end{array}$ & 100 & 1 & 1 & 1 & 12 & 0 & 12 & 24 \\
\hline $\begin{array}{l}\text { Variable } 246 \text { (cols } 491 \text { \& } \\
492 \text { ) }\end{array}$ & $\begin{array}{r}66.6666 \\
7\end{array}$ & $\begin{array}{r}0.60493 \\
8\end{array}$ & 0.609756 & 0.621399 & 8 & 4 & 12 & 24 \\
\hline $\begin{array}{l}\text { Variable } 247 \text { (cols } 493 \& \text { \& } \\
494 \text { ) }\end{array}$ & 100 & 1 & 1 & 1 & 12 & 0 & 12 & 24 \\
\hline $\begin{array}{l}\text { Variable } 248 \text { (cols } 495 \& \\
496 \text { ) }\end{array}$ & $\begin{array}{r}83.3333 \\
3\end{array}$ & 0.4 & 0.428571 & 0.425 & 10 & 2 & 12 & 24 \\
\hline $\begin{array}{l}\text { Variable } 249 \text { (cols } 497 \text { \& } \\
498 \text { ) }\end{array}$ & $\begin{array}{r}91.6666 \\
7\end{array}$ & 0.04348 & 0 & 0 & 11 & 1 & 12 & 24 \\
\hline $\begin{array}{l}\text { Variable } 250 \text { (cols } 499 \text { \& } \\
500)\end{array}$ & $\begin{array}{r}91.6666 \\
7\end{array}$ & $\begin{array}{r}0.90438 \\
2\end{array}$ & 0.904762 & 0.908367 & 11 & 1 & 12 & 24 \\
\hline $\begin{array}{l}\text { Variable } 251 \text { (cols } 501 \& \text { \& } \\
502 \text { ) }\end{array}$ & 100 & 1 & 1 & 1 & 12 & 0 & 12 & 24 \\
\hline $\begin{array}{l}\text { Variable } 252 \text { (cols } 503 \text { \& } \\
504 \text { ) }\end{array}$ & 100 & 1 & 1 & 1 & 12 & 0 & 12 & 24 \\
\hline Variable 253 (cols 505 \& & 100 & 1 & 1 & 1 & 12 & 0 & 12 & 24 \\
\hline
\end{tabular}




\begin{tabular}{|c|c|c|c|c|c|c|c|c|}
\hline & \begin{tabular}{|l|} 
Percent \\
Agreement
\end{tabular} & Scott's Pi & Cohen's Kappa & \begin{tabular}{|l|} 
Krippendorf's \\
Alpha
\end{tabular} & \begin{tabular}{|l|}
$\mathrm{N}$ \\
Agreements
\end{tabular} & \begin{tabular}{|l|}
$N$ \\
Disagreements
\end{tabular} & N Cases & N Decisions \\
\hline \multicolumn{9}{|l|}{ 506) } \\
\hline $\begin{array}{l}\text { Variable } 254 \text { (cols } 507 \& \\
508 \text { ) }\end{array}$ & 100 & 1 & 1 & 1 & 12 & 0 & 12 & 24 \\
\hline $\begin{array}{l}\text { Variable } 255 \text { (cols } 509 \& \\
510)\end{array}$ & 100 & 1 & 1 & 1 & 12 & 0 & 12 & 24 \\
\hline $\begin{array}{l}\text { Variable } 256 \text { (cols } 511 \text { \& } \\
512 \text { ) }\end{array}$ & 100 & 1 & 1 & 1 & 12 & 0 & 12 & 24 \\
\hline $\begin{array}{l}\text { Variable } 257 \text { (cols } 513 \text { \& } \\
514 \text { ) }\end{array}$ & 100 & 1 & 1 & 1 & 12 & 0 & 12 & 24 \\
\hline $\begin{array}{l}\text { Variable } 258 \text { (cols } 515 \text { \& } \\
516 \text { ) }\end{array}$ & 100 & 1 & 1 & 1 & 12 & 0 & 12 & 24 \\
\hline $\begin{array}{l}\text { Variable } 259 \text { (cols } 517 \text { \& } \\
518 \text { ) }\end{array}$ & 100 & 1 & 1 & 1 & 12 & 0 & 12 & 24 \\
\hline $\begin{array}{l}\text { Variable } 260 \text { (cols } 519 \& \\
520 \text { ) }\end{array}$ & $\begin{array}{r}91.6666 \\
7\end{array}$ & 0.04348 & 0 & 0 & 11 & 1 & 12 & 24 \\
\hline $\begin{array}{l}\text { Variable } 261 \text { (cols } 521 \text { \& } \\
522 \text { ) }\end{array}$ & 100 & 1 & 1 & 1 & 12 & 0 & 12 & 24 \\
\hline $\begin{array}{l}\text { Variable } 262 \text { (cols } 523 \text { \& } \\
524 \text { ) }\end{array}$ & 100 & 1 & 1 & 1 & 12 & 0 & 12 & 24 \\
\hline $\begin{array}{l}\text { Variable } 263 \text { (cols } 525 \& \text { } \\
526 \text { ) }\end{array}$ & 100 & 1 & 1 & 1 & 12 & 0 & 12 & 24 \\
\hline $\begin{array}{l}\text { Variable } 264 \text { (cols } 527 \& \\
528)\end{array}$ & 100 & 1 & 1 & 1 & 12 & 0 & 12 & 24 \\
\hline $\begin{array}{l}\text { Variable } 265 \text { (cols } 529 \& \\
530)\end{array}$ & 100 & 1 & 1 & 1 & 12 & 0 & 12 & 24 \\
\hline $\begin{array}{l}\text { Variable } 266 \text { (cols } 531 \& \\
532 \text { ) }\end{array}$ & 100 & 1 & 1 & 1 & 12 & 0 & 12 & 24 \\
\hline $\begin{array}{l}\text { Variable } 267 \text { (cols } 533 \& \text { } \\
534 \text { ) }\end{array}$ & $\begin{array}{r}91.6666 \\
7\end{array}$ & 0.04348 & 0 & 0 & 11 & 1 & 12 & 24 \\
\hline $\begin{array}{l}\text { Variable } 268 \text { (cols } 535 \& \text { } \\
536 \text { ) }\end{array}$ & 100 & 1 & 1 & 1 & 12 & 0 & 12 & 24 \\
\hline $\begin{array}{l}\text { Variable } 269 \text { (cols } 537 \& \\
538 \text { ) }\end{array}$ & 100 & 1 & 1 & 1 & 12 & 0 & 12 & 24 \\
\hline $\begin{array}{l}\text { Variable } 270 \text { (cols } 539 \& \\
540 \text { ) }\end{array}$ & 100 & 1 & 1 & 1 & 12 & 0 & 12 & 24 \\
\hline
\end{tabular}




\begin{tabular}{|c|c|c|c|c|c|c|c|c|}
\hline & \begin{tabular}{|l|} 
Percent \\
Agreement
\end{tabular} & Scott's Pi & Cohen's Kappa & $\begin{array}{l}\text { Krippendorff's } \\
\text { Alpha }\end{array}$ & $\begin{array}{l}\mathrm{N} \\
\text { Agreements }\end{array}$ & $\mid \begin{array}{l}\mathrm{N} \\
\text { Disagreements }\end{array}$ & N Cases & N Decisions \\
\hline $\begin{array}{l}\text { Variable } 271 \text { (cols } 541 \text { \& } \\
542 \text { ) }\end{array}$ & $\begin{array}{r}91.6666 \\
7\end{array}$ & 0.04348 & 0 & 0 & 11 & 1 & 12 & 24 \\
\hline $\begin{array}{l}\text { Variable } 272 \text { (cols } 543 \text { \& } \\
544 \text { ) }\end{array}$ & $\begin{array}{r}91.6666 \\
7\end{array}$ & 0.04348 & 0 & 0 & 11 & 1 & 12 & 24 \\
\hline $\begin{array}{l}\text { Variable } 273 \text { (cols } 545 \text { \& } \\
546 \text { ) }\end{array}$ & 100 & 1 & 1 & 1 & 12 & 0 & 12 & 24 \\
\hline $\begin{array}{l}\text { Variable } 274 \text { (cols } 547 \& \\
548 \text { ) }\end{array}$ & 100 & 1 & 1 & 1 & 12 & 0 & 12 & 24 \\
\hline $\begin{array}{l}\text { Variable } 275 \text { (cols } 549 \text { \& } \\
550 \text { ) }\end{array}$ & 100 & 1 & 1 & 1 & 12 & 0 & 12 & 24 \\
\hline $\begin{array}{l}\text { Variable } 276 \text { (cols } 551 \text { \& } \\
552 \text { ) }\end{array}$ & $\begin{array}{r}91.6666 \\
7\end{array}$ & $\begin{array}{r}0.79831 \\
9\end{array}$ & 0.8 & 0.806723 & 11 & 1 & 12 & 24 \\
\hline $\begin{array}{l}\text { Variable } 277 \text { (cols } 553 \text { \& } \\
554 \text { ) }\end{array}$ & $\begin{array}{r}83.3333 \\
3\end{array}$ & $\begin{array}{r}0.68421 \\
1\end{array}$ & 0.688312 & 0.697368 & 10 & 2 & 12 & 24 \\
\hline $\begin{array}{l}\text { Variable } 278 \text { (cols } 555 \text { \& } \\
556 \text { ) }\end{array}$ & $\begin{array}{r}91.6666 \\
7\end{array}$ & $\begin{array}{r}0.46666 \\
7\end{array}$ & 0.478261 & 0.488889 & 11 & 1 & 12 & 24 \\
\hline $\begin{array}{l}\text { Variable } 279 \text { (cols } 557 \& \\
558 \text { ) }\end{array}$ & $\begin{array}{r}91.6666 \\
7\end{array}$ & $\begin{array}{r}0.71764 \\
7\end{array}$ & 0.72093 & 0.729412 & 11 & 1 & 12 & 24 \\
\hline $\begin{array}{l}\text { Variable } 280 \text { (cols } 559 \& \\
560 \text { ) }\end{array}$ & 100 & 1 & 1 & 1 & 12 & 0 & 12 & 24 \\
\hline $\begin{array}{l}\text { Variable } 281 \text { (cols } 561 \& \text { } \\
562 \text { ) }\end{array}$ & 100 & 1 & 1 & 1 & 12 & 0 & 12 & 24 \\
\hline $\begin{array}{l}\text { Variable } 282 \text { (cols } 563 \& \\
564 \text { ) }\end{array}$ & 100 & 1 & 1 & 1 & 12 & 0 & 12 & 24 \\
\hline $\begin{array}{l}\text { Variable } 283 \text { (cols } 565 \& \\
566 \text { ) }\end{array}$ & 100 & 1 & 1 & 1 & 12 & 0 & 12 & 24 \\
\hline $\begin{array}{l}\text { Variable } 284 \text { (cols } 567 \& \text { } \\
568 \text { ) }\end{array}$ & 100 & 1 & 1 & 1 & 12 & 0 & 12 & 24 \\
\hline $\begin{array}{l}\text { Variable } 285 \text { (cols } 569 \text { \& } \\
570 \text { ) }\end{array}$ & 100 & 1 & 1 & 1 & 12 & 0 & 12 & 24 \\
\hline $\begin{array}{l}\text { Variable } 286 \text { (cols } 571 \& \\
572 \text { ) }\end{array}$ & $\begin{array}{r}83.3333 \\
3\end{array}$ & $\begin{array}{r}0.63909 \\
8\end{array}$ & 0.641791 & 0.654135 & 10 & 2 & 12 & 24 \\
\hline $\begin{array}{l}\text { Variable } 287 \text { (cols } 573 \& \text { \& } \\
574 \text { ) }\end{array}$ & 75 & $\begin{array}{r}0.56886 \\
2\end{array}$ & 0.571429 & 0.586826 & 9 & 3 & 12 & 24 \\
\hline Variable 288 (cols 575 \& & 91.6666 & & 0 & 0 & 11 & 1 & 12 & 24 \\
\hline
\end{tabular}




\begin{tabular}{|c|c|c|c|c|c|c|c|c|}
\hline & \begin{tabular}{|l|} 
Percent \\
Agreement
\end{tabular} & Scott's Pi & Cohen's Kappa & \begin{tabular}{|l|} 
Krippendorf's \\
Alpha
\end{tabular} & \begin{tabular}{|l|l|}
$\mathrm{N}$ \\
Agreements
\end{tabular} & $\begin{array}{l}\mathrm{N} \\
\text { Disagreements }\end{array}$ & N Cases & N Decisions \\
\hline 576) & 7 & 0.04348 & & & & & & \\
\hline $\begin{array}{l}\text { Variable } 289 \text { (cols } 577 \text { \& } \\
578 \text { ) }\end{array}$ & 100 & 1 & 1 & 1 & 12 & c & 12 & 24 \\
\hline $\begin{array}{l}\text { Variable } 290 \text { (cols } 579 \& \\
580 \text { ) }\end{array}$ & 100 & 1 & 1 & 1 & 12 & c & 12 & 24 \\
\hline $\begin{array}{l}\text { Variable } 291 \text { (cols } 581 \text { \& } \\
582 \text { ) }\end{array}$ & $\begin{array}{r}66.6666 \\
7\end{array}$ & 0.54067 & 0.542857 & 0.559809 & 8 & 4 & 12 & 24 \\
\hline $\begin{array}{l}\text { Variable } 292 \text { (cols } 583 \& \\
584 \text { ) }\end{array}$ & $\begin{array}{r}91.6666 \\
7\end{array}$ & $\begin{array}{r}0.88940 \\
1\end{array}$ & 0.889908 & 0.894009 & 11 & 1 & 12 & 24 \\
\hline $\begin{array}{l}\text { Variable } 293 \text { (cols } 585 \text { \& } \\
586 \text { ) }\end{array}$ & 100 & 1 & 1 & 1 & 12 & c & 12 & 24 \\
\hline $\begin{array}{l}\text { Variable } 294 \text { (cols } 587 \text { \& } \\
588 \text { ) }\end{array}$ & $\begin{array}{r}91.6666 \\
7\end{array}$ & $\begin{array}{r}0.74736 \\
8\end{array}$ & 0.75 & 0.757895 & 11 & 1 & 12 & 24 \\
\hline $\begin{array}{l}\text { Variable } 295 \text { (cols } 589 \& \\
590 \text { ) }\end{array}$ & $\begin{array}{r}91.6666 \\
7\end{array}$ & $\begin{array}{r}0.74736 \\
8\end{array}$ & 0.75 & 0.757895 & 11 & 1 & 12 & 24 \\
\hline$* * *$ & & & $\begin{array}{l}\text { Krippendorf's } \\
\text { Alpha }\end{array}$ & 0.851383 & & & & \\
\hline
\end{tabular}

\section{References}

Adhikari, A., Emerson, D., Gouldman, A. \& Tondkar, R. (2015). An examination of corporate social disclosure of multinational corporations: A cross-national investigation. Advances in Accounting, Incorporating Advances in International Accounting, 31: 100-106.

Aktar, W., Sengupta, D. \& Chowdhury, A. (2009). Impacts of pesticides use in agriculture: Their benefits and hazards. Interdisciplinary Toxicology, 2(1): 1-12.

Ali, W., Frynas, J. G., \& Mahmood, Z. (2017). Determinants of corporate social responsibility (CSR) disclosure in developed and developing countries: A literature review. Corporate Social Responsibility and Environmental Management, 24(4): 273-294. 
Alrazi, B., de Villiers, C., van Staden, C. J. (2015). A comprehensive literature review on, and the construction of a framework for, environmental legitimacy, accountability and proactivity. Journal of Cleaner Production, 102: 44-57.

Al-Shaer, H. (2014). The relationship between audit committees, corporate environmental disclosure, and environmental reputation: UK evidence. Doctoral thesis. Durham University.

Arora, M. P., \& Lodhia, S. (2017). The BP Gulf of Mexico oil spill: Exploring the link between social and environmental disclosure and reputation risk management. Journal of Cleaner Production, 140(3): 1287-1297.

Ashforth, B. E., \& Gibbs, B. W. (1990). The double-edge of organizational legitimation. Organization Science, 1(2), 177-194.

Beck, A. C., Campbell, D. \& Shrives, P. J. (2010). Content analysis in environmental reporting research: Enrichment and rehearsal of the methods in a British-German context. The British Accounting Review, 42(31): 207-222.

Bhandari, G. (2014). An overview of agrochemicals and their effects on the environment in Nepal. Applied Ecology and Environmental Sciences, 2(2): 66-73.

Borenstein, S. (2012, Mar 30). Common pesticide makes honeybees get lost and reduces bumblebee hive weight, new studies say. Whitehorse Star Retrieved from http://ezproxy.lib.ryerson.ca/login?url=https://search-proquestcom.ezproxy.lib.ryerson.ca/docview/964041615?accountid=13631 [Accessed July 9, 2018]

Bowen, H.R. (1953). Social responsibilities of the businessman. New York, NY: Harper.

Braam, G., Weerd, L., Hauck, M., \& Huijbregts, M. (2016). Determinants of corporate environmental reporting: the importance of environmental performance and assurance. Journal of Cleaner Production, 129: 724-734.

Broughton, E. (2005). The Bhopal disaster and its aftermath: A review. Environmental Health, 4(6): 1-6.

Buhr, N., \& Freedman, M. (2001). Culture, institutional factors and differences in environmental disclosure between Canada and the United States. Critical Perspectives on Accounting, 12: 293322.

Carrol, A. B. (1999). Corporate social responsibility. Business \& Society, 38(3): 268-295.

Chaklader, B. \& Gulati, P. A. (2015). A study of corporate environmental disclosure practices of companies doing business in India. Global Business Review, 16(2): 321-335.

Cho, C. H. \& Patten, D. M. (2007). The role of environmental disclosures as tools of legitimacy: A research note. Accounting, Organizations and Society, 32: 639-647.

Chouhan, T. R. (2005). The unfolding of Bhopal disaster. Journal of Loss Prevention in the Process Industries, 18: 205-208.

Christmann, P. (2004). Multinational companies and the natural environment: Determinants of global environmental policy standardization. Academy of Management Journal, 47(5): 747-760. 
The Companies Act. (2013). Government of India: Ministry of Corporate Affairs. Retrieved from http://www.mca.gov.in/Ministry/pdf/CompaniesAct2013.pdf [Accessed April 24th, 2018]

Cormier, D., \& Magnan, M. (1999). Corporate environmental disclosure strategies: Determinants, costs and benefits. Journal of Accounting, Auditing and Finance, 14(4): 429-451.

Darus, F., Arshad, R., Othman, S., \& Jusoff, K. (2009). Influence of institutional pressure and ownership structure on corporate social responsibility disclosure. Interdisciplinary Journal of Contemporary Research in Business, 1(5), 123-150.

Davis, D., Bell, M. \& Fletcher, T. (2002). A look back at the London smog of 1952 and the half century since. Environmental Health Perspectives, 110(12): A734-A735.

De Swert K. (2012). Calculating inter-coder reliability in media content analysis using Krippendorff's Alpha. Amsterdam University. Available at http:// www.polcomm.org/wpcontent/uploads/ICR01022012.pdf [Accessed April 30 $\left.{ }^{\text {th }}, 2018\right]$.

Deegan, C., Rankin, M. \& Tobin, J. (2002). An examination of the corporate social and environmental disclosures of BHP from 1983-1997: A test of legitimacy theory. Accounting, Auditing \& Accountability Journal, 15(3): 312-343.

Deephouse, D. L., Bundy, J., Plunkett Tost, L., \& Suchman, M. C. (2017). Organizational Legitimacy : Six Key Questions. In R. Greenwood, C. Oliver, T. Lawrence, \& R. Meyer (Eds.), The SAGE Handbook of Organizational Institutionalism (2nd ed.) (pp. 1-42). Thousand Oaks CA: Sage.

Deodhar, S.Y. (2016). Trapping India's CSR in a legal net: Will the mandatory trusteeship contribute to triple bottom line? The Journal for Decision Makers, 41(4): 267-247.

Donovan, G. O. (2002). Environmental disclosures in the annual report: Extending the applicability and predictive power of legitimacy theory. Accounting, Auditing \& Accountability Journal, 15(3), 344-371.

Dowling, J., \& Pfeffer, J. (1975). Organizational legitimacy: Social values and organizational behavior. The Pacific Sociological Review, 18(1), 122-136.

Endrikat, J., Guenther, E., \& Hoppe, H. (2014). Making sense of conflicting empirical findings: A metaanalytic review of the relationship between corporate environmental and financial performance. European Management Journal, 32(5): 735-751.

Ezhilarasi, G., \& Kabra, K. C. (2017). Factors influencing environmental disclosures: Evidence from India. IUP Journal of Accounting Research \& Audit Practices, 16(1), 7-24.

Fiaschi, D., Giuliani, E. \& Nieri, F. (2014). BRIC companies seeking legitimacy through corporate social responsibility. Discussion paper. University of Pisa.

Fifka, M. S. (2013). Corporate responsibility reporting and its determinants in comparative perspective a review of the empirical literature and meta-analysis. Business Strategy and the Environment, 22(1): 1-35.

Gibson, K. \& O'Donovan, G. (2007). Corporate governance and environmental reporting: an Australian study. Corporate Governance: An International Review, 15(5): 944-956. 
Hahn, R., \& Lülfs, R. (2014). Legitimizing Negative Aspects in GRI-Oriented Sustainability Reporting: A Qualitative Analysis of Corporate Disclosure Strategies. Journal of Business Ethics, 123(3), 401420.

Harada, M. (1995). Minamata disease: Methylmercury poisoning in Japan caused by environmental pollution. Critical Reviews in Toxicology, 25(1): 1-24.

Haron, A. J. (2016). Standardized versus localized strategy: The role of cultural patterns in society on consumption and market research. Journal of Accounting \& Marketing, 5(1): 1-4.

Hall, R. (2014). Sustainability reporting within the food industry. Master's Thesis. Swedish University of Agricultural Sciences.

Hayes, T.B., Hasen, M. (2017). From silent spring to silent night: Agrochemicals and the Anthropocene. Elementa: Science of the Anthropocene, 5: 57.

Henry, M., Beguin, M., Requier, F., Rollin, O., Odoux, J.F., Aupinel, P., Aptel. J., Tchamitchain, S., \& Decourtye, A. (2012). A common pesticide decreases foraging success and survival in honey bees. Science, 336 (6079): 348-350.

Hossain, M. \& Andrew, J. (2006). Corporate social and environmental disclosure in developing countries: Evidence from Bangladesh. University of Wollongong Research.

Hughes, A., Anderson, A. \& Golden, S. (2001). Corporate environmental disclosure: Are they useful in determining environmental performance? Journal of Accounting \& Public Policy, 20(3): 217-240.

Hu, Y. Y. \& Karbharl, Y. (2015). Incentives and disincentives of corporate environmental disclosure: Evidence from listed companies in China and Malaysia. Thunderbird International Business Review, 57(2): 143-161.

Hummel, K. \& Schlick, C. (2016). The relationship between sustainability performance and sustainability disclosure- Reconciling voluntary disclosure theory and legitimacy theory. Journal of Accounting and Public Policy, 35(5): 455-476.

Hunter, T. \& Bansal, P. (2007). How standard is standardization MNC global environmental communication? Journal of Business Ethics, 71(2): 135-147.

Ingram, R. \& Frazier, K. (1980). Environmental performance and corporate disclosure. Journal of Accounting Research, 18(2): 614-622.

Ioannou, I., \& Serafeim, G. (2017). The consequences of mandatory corporate sustainability reporting. Harvard Business School Research Working Paper, 11-100.

Khan, F. I. \& Abbasi. S. A. (1999). Major accident in process industries and an analysis of causes and consequences. Journal of Loss Prevention in the Process Industries, 12: 361-378.

Khan, A., Muttakin, M. B. \& Siddiqui, J. (2013). Corporate governance and corporate social responsibility disclosures: Evidence from an emerging economy. Journal of Business Ethics, 114(2): 207-223.

Khlif, H., Ahmed, K., \& Souissi, M. (2017). Ownership structure and voluntary disclosure: A synthesis of empirical studies. Australian Journal of Management, 42(3): 376-403.

Kolk, A. \& Pinkse, J. (2010). The integration of corporate governance in corporate social responsibility disclosures. Corporate Social Responsibility and Environmental Management 17(1): 15-26. 
Kostova, T. \& Zaheer, S. (1999). Organizational legitimacy under conditions of complexity: The case of the multinational enterprise. The Academy of Management Review, 24(1): 64-81.

KPMG. (2011). International survey of corporate responsibility reporting 2011. Amsterdam: KPMG.

KPMG. (2016). Indian agrochemical industry prepares for high growth. Reaction, 21:14-23.

KPMG. (2017). India's CSR reporting survey 2016. Amsterdam: KPMG.

Kshetrimayum, R. \& Naorem, P. (2015). Multinational companies in India- An analysis. Sai Om Journal of Commerce \& Management, 2(1): 9-14.

Lin, L. W. (2008). Corporate social and environmental disclosure in emerging securities markets: China as a case study. Master's Thesis. University of Illinois.

Long, B. (2000). International environmental issues and the OECD 1950-2000: An historical perspective. OECD Publishing: Paris, France.

Lu, Y. \& Abeysekera, I. (2014). Stakeholders' power, corporate characteristics, and social and environmental disclosure: evidence from China. Journal of Cleaner Production, 64: 426-436.

Luo, L., Tang, Q. \& Yi-Chen, L. (2013). Comparison of propensity for carbon disclosure between developing and developed countries. Accounting Research Journal, 26(1), 6-34.

Malarvizhi, P. (2008). Corporate environmental disclosures on the internet: An empirical analysis of Indian companies. Issues in Social and Environmental Accounting, 2(2): 211-232.

Marcott, J. (2012, Apr 6). Studies fault Bayer in bee die-off. Minneapolis Star Tribune Retrieved from https://www.csmonitor.com/Science/2012/0406/Studies-fault-Bayer-in-bee-die-off [Accessed July 9, 2018]

Mathews, M. R. (1997). Twenty-five years of social and environmental accounting research: Is there a silver jubilee to celebrate? Auditing \& Accountability Journal, 10(4): 481-531. doi: 10.1108/EUM0000000004417143.

Menassa, E. \& Brodhäcker, M. (2017). The type and quantity of corporate social disclosure of German "universal" banks. Journal of Management \& Governance, 21(1): 119-143.

Meng, X., Zeng, S., Shi, J. J., Qi, G. Y. \& Zhang, Z. B. (2014). The relationship between corporate environmental performance and environmental disclosure: An empirical study in China. Journal of Environmental Management, 145: 357-367.

Mew, E. J., Padmanathan, P., Konradsen, F., Eddleston, M., Chang, A., Phillips, M., \& Gunnell, D. (2017). Journal of Affective Disorders, 219(2017): 93-104.

Momin, M. (2006). Corporate social responsibility and reporting by multinational corporations in Bangladesh: An exploration. Doctoral thesis. University of Glasgow.

Monteiro, S., \& Aibar-Guzmán, B. (2010). Determinants of environmental disclosure in the annual reports of large companies operating in Portugal. Corporate Social Responsibility and Environmental Management, 17(4): 185-204.

Mousa, G. A. \& Hassan, N. T. (2015). Legitimacy theory and environmental practices: Short notes. International Journal of Business and Statistica Analysis, 2(1): 41-53. 
Oh, W., Change, Y.K. \& Martynov, A. (2011). The effects of ownership structure on corporate social responsibility: Empirical evidence from Korea. Journal of Business Ethics, 104(2): 283-297.

Ortas, E., Gallego-Alvarez, I. \& Etxeberria, I. A. (2014). Financial factors influencing the quality of corporate social responsibility and environmental management disclosure: A quantile regression approach. Corporate Social Responsibility and Environmental Management, 22(6): 362-380.

Özen, S., \& Küskü, F. (2009). Corporate environmental citizenship variation in developing countries: An institutional framework. Journal of Business Ethics, 89(2): 297-313.

Park, B. I. \& Ghauri, P. N. (2015). Determinants influencing CSR practices in small and medium sized MNE subsidiaries: A stakeholder perspective. Journal of World Business, 50:192-204.

Qiu, Y., Shaukat, A., Tharyan, R. (2016). Environmental and social disclosures: Link with corporate financial performance. The British Accounting Review, 48(1): 102-116.

Quinteros, E., Ribó, A., Mejía, R., López, A., Belteton, W., Comandari, A., Orantes, C. M., Pleites, E. B., Hernández, C. E. \& López, D. L. (2017). Heavy metals and pesticide exposure from agricultural activities and former agrochemical factory in a Salvadoran rural community. Environmental Science and Pollution Research, 24(2): 1662-1676.

Research and Markets. (2016). Global agrochemical market: Trends analysis \& forecasts to 2021. Available at https://www.researchandmarkets.com/research/jmv559/global [Accessed October 3rd, 2017].

Riaz, Z., Ray, S., Ray, P. K. \& Kumar, V. (2015). Disclosure practices of foreign and domestic firms in Australia. Journal of World Business, 50(1): 781-792.

Rockness, J. W. (1985). An assessment of the relationship between US corporate environmental performance and disclosure. Journal of Business Finance and Accounting, 12(3): 339-354.

Setyorini, C. T. \& Ishak, Z. (2012). Corporate social and environmental disclosure: A positive accounting theory viewpoint. International Journal of Business and Social Science, 3(9): 152-164.

Samal, N., Saha, P., Roy, P., Biswasroy, M., Ramana, R. V. \& Mazumdar, A. (2011). Limnological analysis of an urban polluted lake in Bangalore city in India. Desalination and Water Treatment, 30(1-3): 217-228.

Sen, M., Mukherjee, K., \& Pattanayak, J. K. (2011). Corporate environmental disclosure practices in India. Journal of Applied Accounting Research, 12(2): 139-156. doi:10.1108/09675421111160709.

Sparks, T. \& Lorbach, B. (2017). Perspective on the agrochemical industry and agrochemical discovery. Pest Management Science, 73(4): 672-677.

Srivastav, S. K., Reddy, C. S. S., Bhattacharya, A., \& Reddy, P. R. (1998). Technical note monitoring the Oil \& Natural Gas Corporation (ONGC) oil well fire at Pasarlapudi, Andhra Pradesh, India. International Journal of Remote Sensing, 19(18): 3515-3517.

Stix, G. (1989). Bhopal: A tragedy in waiting. IEEE Spectrum, 26(6): 47-50. doi: 10.1109/6.29340.

Stubbs, W., Higgins, C., \& Milne, M. (2013). Why do companies not produce sustainability reports? Business Strategy and the Environment, 22(7): 456-470.

Suchman, M. (1995). Managing legitimacy: Strategic and institutional approaches. The Academy of Management Review, 20(3): 571-610. 
Suddaby, R., Bitektine, A. \& Haack, P. (2017). Legitimacy. Academy of Management Annals, 11(1): 451478.

Sumiani, Y., Haslinda, Y., \& Lehman, G. (2007). Environmental reporting in a developing country: a case study on status and implementation in Malaysia. Journal of Cleaner Production, 15 (2007): 895901.

Tilt, C. A. (2001). The content and disclosure of Australian corporate environmental policies. Accounting, Auditing \& Accountability Journal, 142 (2), 190-212.

Trabelsi, S., Debreceny, R., \& Lymer, A. (2013). An empirical examination of corporate websites as a voluntary disclosure medium. International Journal of Applied Decision Sciences, 7 (1): 1-32.

Unsworth. J. B., Shakil, N. A., Kumar, G. A., Kelter, G. A. \& Linders, J. B. H. J. (2012). Agrochemicals. Chemistry International, 34 (4): 34-36.

Villiers, C. \& van Staden, C. J. (2011). Where firms choose to disclose voluntary environmental information. Journal of Accounting and Public Policy, 30: 504-525.

Wang, J., Tian, G., Fan, W., \& Luo, D. (2017). The effects of mandatory regulation on corporate social responsibility reporting quality: Evidence from China. Journal of Applied Business Research, 33(1): 67-86.

Weber, R. P. (1990). Basic content analysis: Second edition. Sage Publications, Inc: Newbury Park, California.

Whitehorn, P., O'Connor, S., Wackers, F., Goulson, D. (2012). Neonicotinoid pesticide reduces bumble bee colony growth and queen production. Science, 336(6079): 351-352.

Wilson, N. A. (2013). Localization of standardization? A comparative analysis of multinational agrochemical corporations' environmental disclosure practices in India. Masters thesis. Ryerson University.

Yang, M., Khan, F. \& Amyotte, P. (2014). Operational risk assessment: A case of the Bhopal disaster. Process Safety and Environmental Protection, 97: 70-79.

Zeng, S. X., Xu, X. D., Yin, H. T., \& Tam, C. M. (2012). Factors that drive Chinese listed companies in voluntary disclosure of environmental information. Journal of Business Ethics, 109(3): 309-321. 